\title{
Current-induced spin-orbit torques in ferromagnetic and antiferromagnetic systems
}

\author{
A. Manchon* \\ King Abdullah University of Science and Technology (KAUST), \\ Physical Science and Engineering Division (PSE), \\ and Computer, Electrical, \\ and Mathematical Science and Engineering (CEMSE), \\ Thuwal, 23955-6900, \\ Saudi Arabia \\ I. M. Miron \\ University of Grenoble Alpes, \\ CNRS, CEA, INAC-SPINTEC, \\ F-38000 Grenoble, \\ France \\ T. Jungwirth \\ Institute of Physics, \\ Academy of Sciences of the Czech Republic, 16200 Praha, \\ Czech Republic \\ School of Physics and Astronomy, \\ University of Nottingham, \\ Nottingham NG7 2RD, \\ United Kingdom \\ J. Sinova \\ Institut für Physik, \\ Johannes Gutenberg Universität Mainz, \\ 55128 Mainz Germany \\ Institute of Physics, \\ Academy of Sciences of the Czech Republic, 16200 Praha, \\ Czech Republic \\ J. Železný \\ Institute of Physics, \\ Academy of Sciences of the Czech Republic, 16200 Praha, \\ Czech Republic
}

A. Thiaville

Laboratoire de Physique des Solides,

Univ. Paris-Sud,

CNRS UMR 8502 - 91405 Orsay Cedex,

France

K. Garello

IMEC, Kapeeldreef 75, 3001 Leuven,

Belgium

P. Gambardella

Department of Materials,

ETH Zürich, Hönggerbergring 64,

$\mathrm{CH}$-8093 Zürich,

Switzerland

(Dated: January 30, 2018)

Spin-orbit coupling in inversion-asymmetric magnetic crystals and structures has emerged as a powerful tool to generate complex magnetic textures, interconvert charge and spin under applied current, and control magnetization dynamics. Current-induced 
spin-orbit torques mediate the transfer of angular momentum from the lattice to the spin system, leading to sustained magnetic oscillations or switching of ferromagnetic as well as antiferromagnetic structures. The manipulation of magnetic order, domain walls and skyrmions by spin-orbit torques provides evidence of the microscopic interactions between charge and spin in a variety of materials and opens novel strategies to design spintronic devices with potentially high impact in data storage, nonvolatile logic, and magnonic applications. This paper reviews recent progress in the field of spin-orbitronics, focusing on theoretical models, material properties, and experimental results obtained on bulk noncentrosymmetric conductors and multilayer heterostructures, including metals, semiconductors, and topological insulator systems. Relevant aspects for improving the understanding and optimizing the efficiency of nonequilibrium spin-orbit phenomena in future nanoscale devices are also discussed.

\section{CONTENTS}

I. Introduction

II. Overview

A. Magnetization dynamics induced by spin-orbit torque

B. Spin-orbit torque in antiferromagnets

C. Topology and spin-orbit torque

D. Inverse effect of the spin-orbit torque

III. Theory of spin-orbit torques

A. Kubo linear response: intraband versus interband transitions

B. Symmetry of spin-orbit torques

C. Spin Hall effect spin-orbit torque

D. Spin-orbit torque in two- and three-dimensional bulk systems

1. Mechanisms, components, and symmetries

2. Inverse spin galvanic torque in a magnetic $2 \mathrm{D}$ electron gas

3. Non-centrosymmetric bulk magnets

E. Microscopic modeling of spin-orbit torques in bilayer systems

F. Antiferromagnets

G. Three-dimensional topological insulators

H. Other spin-orbit torques

1. Anisotropic magnetic tunnel junctions

2. Two dimensional topological insulators and hexagonal lattices

3. Spin-transfer torque assisted by spin-orbit coupling

IV. Spin-orbit torques in magnetic multilayers

A. Phenomenological description

B. Measurement techniques

1. Harmonic Hall voltage analysis

2. Spin-torque ferromagnetic resonance

3. Magneto-optic Kerr effect

C. Materials survey

1. Metal multilayers

2. FM/semiconductor layers

3. Surface alloys and two-dimensional gases

4. ferromagnet/topological insulator layers

D. Magnetization dynamics

E. Magnetization switching

1. SOT switching mechanism

2. Switching speed

3. Zero field switching

F. Memory and logic devices
V. Spin-orbit torque in noncentrosymmetric magnets

A. Non-magnetic GaAs structures

B. Bulk ferromagnetic $(\mathrm{Ga}, \mathrm{Mn}) \mathrm{As}$ and $\mathrm{NiMnSb}$

C. Collinear antiferromagnets

D. Antiferromagnetic topological Dirac fermions

E. Magnonic charge pumping in $(\mathrm{Ga}, \mathrm{Mn}) \mathrm{As}$

VI. Spin-orbit torques and magnetic textures

A. Domain wall dynamics under current

B. In-plane magnetized samples

1. Soft samples (X domains)

2. Anisotropic samples with Y domains

C. Perpendicularly magnetized samples

1. Demonstrations of spin-orbit torques in current-induced domain wall motion

2. Domain wall motion under spin-orbit torque

3. Two-dimensional effects in current-induced domain wall motion

4. Domain wall motion under combined spin transfer and spin-orbit torques

5. Motion of magnetic skyrmions under spin-orbit torques

6. Domain wall motion in disordered media

D. Antiferromagnetic and ferrimagnetic systems

VII. Perspectives

List of abbreviations

56

Acknowledgements

57

References

57

\section{INTRODUCTION}

Magnetic memories use ordered spin states in magnets as a storage medium of digital information (Brataas et al., 2012a; Chappert et al., 2007; Kent and Worledge, 2015). The technology dates back to first attempts of recording sound (Sinova and Jungwirth, 2017) and, presently, magnetic hard-drives provide the virtually unlimited data storage space on the internet (Parkin, 2014). In the future, magnetic computer memories are projected to be among the leading alternatives to complement CMOS in the "beyond Moore's law" information technologies (Waldrop, 2016).

A key field behind the success of hard-drives and the new research directions of magnetic memories is spintronics. It explores possibilities to add spin degree of 
freedom to conventional charge-based microelectronic devices or to completely replace charge with spin functionalities (Bader and Parkin, 2010; Wolf et al., 2001; Zutic et al., 2004). Over the past three decades of research and development, spintronics has offered means to replace magnetic fields for reading and writing information in magnets by more scalable spin-dependent transport phenomena (Brataas et al., 2012a; Chappert et al., 2007; Kent and Worledge, 2015). Among the recently discovered spintronic tools for magnetization switching or, more generally, for exciting magnetization dynamics, spin-orbit torque (SOT) takes a prominent place. SOT relies on the conversion of electrical current to spin (Sinova et al., 2015). It is allowed by the spin-orbit coupling term in the Dirac equation which makes it an abun- dant and potentially highly efficient phenomenon.

SOT in the context of electrical writing in magnetic memories (Fukami and Ohno, 2017; Prenat et al., 2016) is the central topic of this review. The technological relevance of the SOT for the development of the next generation of magnetic random access memories (MRAMs) was pointed out in breakthrough reports by Miron et al., 2011a and Liu et al., 2012b, where they demonstrated SOT switching of the recording magnet. Apart from the application potential, the reports also analyzed the symmetry of the measured SOT and framed the discussion of its microscopic origin in the most commonly used nonmagnetic metal (NM)/FM transition-metal interfaces by considering the following two model scenarios that contribute to the torque:

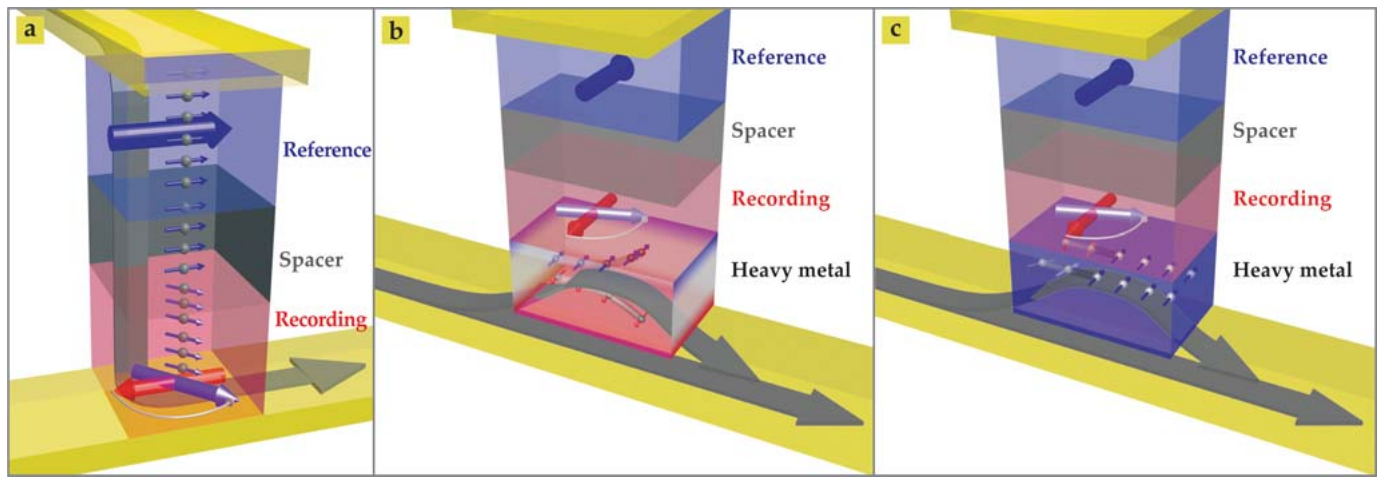

FIG. 1 (Color Online) STT vs. SOT switching of a magnet. (a) In the STT mechanism, a current (gray arrow) of polarized electrons from a reference FM passes down through a spacer into a recording FM. Within a few atomic monolayers of entering the recording magnet, the flowing electrons align with the instantaneous recording magnetization (large purple arrow in the recording medium). This alignment results in a torque (curved white arrow) on the recording FM that ultimately causes the recording magnetization to flip from its original orientation (large red arrow). In the snapshot shown here, the recording magnetization is about $2 / 3$ of the way to being flipped. Note that the time scale for the full reversal is much greater than the time needed for the current to flow from the reference FM through the recording FM. A second mechanism, SOT, can be driven by the SHE or by the iSGE. (b) In the SHE variant, as current flows along the contact and the NM layer, a spin current is generated that flows upward into the recording FM and flips its magnetization. (c) In the iSGE mechanism, electrons become polarized at the interface of a NM heavy metal and a FM; the polarized electrons then switch the magnetization of the recording FM. From Sinova and Jungwirth, 2017.

In one picture, charge current is directly converted to spin accumulation due to spin-orbit coupling at the inversion-symmetry breaking interface, where it exerts the torque on magnetization via the exchange coupling (Manchon and Zhang, 2008). Several names have appeared in the literature for this model mechanism generating spin accumulation that leads to the SOT, such as the Edelstein-Rashba effect (Edelstein, 1990) or the inverse spin galvanic effect (iSGE) (Belkov and Ganichev, 2008). In this review we will use the term to describe this model scenario as the iSGE-SOT.

In the other model scenario, spin-orbit coupling generates a spin current in the NM layer due to the spin Hall effect (SHE) (Sinova et al., 2015). The spin current propagates towards the interface, where it is absorbed in the form of a magnetization torque in the adjacent ferromagnet (FM). The SHE-SOT and iSGE-SOT can act in parallel. This is reminiscent of the early observations in NM semiconductors of the SHE and iSGE as companion phenomena, both allowing for electrical alignment of spins in the same structure (Kato et al., 2004a,b; Wunderlich et al., 2004, 2005).

As illustrated in Fig. 1, the SHE or iSGE induced SOT is fundamentally distinct from the earlier discovered current-induced switching mechanism due to the spintransfer torque (STT) (Ralph and Stiles, 2008). In the STT, spin-polarized electrons are injected from a reference FM layer to an adjacent recording FM film. Via a non-relativistic angular momentum transfer mechanism, the injected spins then exert a torque that switches the magnet. The out-of-plane writing current geometry of this more technologically mature switching principle sets physical limitations on efficiency and endurance of the STT-MRAM cell. These can be overcome by the in-plane 
writing current geometry of the SOT (Fukami and Ohno, 2017; Prenat et al., 2016).

Considering the SOT as originating from either the iSGE or SHE model scenario can provide a useful physical and materials guidance. The necessary condition for the iSGE-induced non-equilibrium spin polarization is the broken inversion symmetry, which is automatically fulfilled in the above mentioned interfaces. However, also uniform crystals can have unit cells that lack a center of symmetry. The initial discovery of the iSGE-SOT was made in such a crystal, namely in the zinc-blende diluted magnetic semiconductor (Ga,Mn)As (Chernyshov et al., 2009) and later also reported in asymmetric metal layers (Miron et al., 2010). This line of research was subsequently extended to crystals whose individual atomic positions in the unit cell are locally non-centrosymmetric. It led to the discovery of a staggered iSGE polarization that, if commensurate with a staggered Néel magnetic order, results in efficient current-induced SOT switching in an antiferromagnet (AF) (Wadley et al., 2016; Železný et al., 2014).

The notion of the SHE-induced SOT, on the other hand, led to systematic studies successfully correlating trends in the magnitude and sign of the SOT with the magnitude and sign of the SHE in the NM spin-orbit coupled material interfaced with the magnet. However, in the commonly used bilayers with a nm-scale spindiffusion length in the NM material and a (sub)nm-thick magnetic film, the distinction between SOTs generated by "bulk"-SHE or "interface"-iSGE remains principally blurred. Moreover, the experimentally observed complex SOT phenomenology in the bilayer structures is often not captured by either of the two idealized model physical scenarios (Garello et al., 2013).

In this article we review present theoretical understanding of the SOT in various types of material systems and summarize the experimentally established SOT phenomenology. We also discuss links of SOT to other currently highly active research fields, such as the topological phenomena in condensed matter, and outline foreseen technological applications of the SOT. A brief overview is given in Section II. Readers interested in a more detailed discussion of theoretical and experimental aspects of SOT are referred to the subsequent sections.

\section{OVERVIEW}

\section{A. Magnetization dynamics induced by spin-orbit torque}

Initially, the damping-like component of the SOT was identified by measuring the damping of the ferromagnetic resonance (FMR) in NM/FM bilayers (Ando et al., 2008). A change of the damping factor was induced in the experiment by an in-plane dc current and interpreted as a consequence of the SHE-SOT. This was a new con- cept in which a dc electrical current driven through a conductor adjacent to the FM controls the FMR damping, in contrast to traditional means of controlling the FMR frequency by the dc current-induced Oersted field. Since the SHE in the NMs was an emerging topic at the time of these pioneering SOT experiments, one of the key perceived merits of the SOT then was in providing an experimental measure of the spin Hall angle in the NM material (Ando et al., 2008). From our present perspective, however, we have to digress here and emphasize that this has to be taken with great caution. The inferred "spin Hall angles" from these experiments are mere effective parameters capturing, besides the bulk spin Hall effect, also the iSGE and other potential spin-orbit coupling and spin-current contributions originating from the spin-orbit coupled from the interface (Amin and Stiles, 2016a,b; Kim et al., 2017b; Lifshits and Dyakonov, 2009; Miron et al., 2011a; Saidaoui and Manchon, 2016; Sinova et al., 2015).

While in experiments pioneered by Ando et al., 2008 the FMR is generated externally and the SOT only modifies the dynamics, Liu et al., 2011 demonstrated that the SOT itself can drive the FMR when an alternating in-plane current is applied to the NM/FM bilayer. The method was again conceived to provide additional means utilizing FM dynamics for measuring the SHE in the adjacent NM layer (Liu et al., 2011). A remarkable turn of events appeared, however. Miron et al., 2011a and, subsequently, Liu et al., 2012b observed that SOT can not only trigger small angle FMR precession but, for large enough electrical currents, it can fully and reversibly switch FM moments. The roles of the FM and NM layers got reversed: In the original experiment by Ando et al., 2008, the FM provided the tool and the relativistic effects in the NM layer were the object of interest. From now on, the new means to manipulate the magnetization took central stage.

Phenomenologically, SHE-SOT may appear as a mere counterpart of the STT (Ando et al., 2008; Liu et al., 2012b). At first sight, the spin current injected from the NM layer due to the SHE just replaces the spin-injection from the reference to the recording FM in the STT stack (see Fig. 1). However, the change in the writing electrical current geometry from out-of-plane in the STT to in-plane in the SOT has major consequences for the operation of memory devices as well as for the transport properties of layered structures.

In the STT, each electron can only transfer one quantum unit of spin angular momentum as it travels from the reference to the recording FM. In the relativistic SOT utilizing no reference spin polarizer, the spin angular momentum generated from the linear momentum in between collisions is small but it gives a little kick in every collision or acceleration that the electron feels. One has replaced a larger one-time push by many small pushes to topple the magnetization. In the SOT case, the net transfer per 
electron is not anymore limited by one quantum unit of spin angular momentum. This fact has opened an entirely new space for material and device optimization of the switching process in SOT MRAMs.

Present MRAM bit cells utilize the tunneling magnetoresistance (TMR) for readout (Chappert et al., 2007). The TMR effect is maximized when the recording and reference magnetizations switch between parallel and antiparallel configurations. For STT writing in such a device, however, the injected spin from the reference FM with a precisely aligned or anti-aligned orientation to the recording magnetization exerts no torque. This implies that the STT mechanism relies on thermal fluctuations of magnetization and the associated incubation time for initializing the magnetization dynamics slows down the switching process. In the SOT, on the other hand, the orientation of the current-induced spin polarization that exerts the torque in the recording FM is independent of the magnetization in the reference FM of the TMR stack, and can be engineered to be misaligned with the recording magnetization. This eliminates the incubation time. Therefore, the in-plane writing current geometry can make the SOT more efficient and faster than STT (Aradhya et al., 2016; Baumgartner et al., 2017; Fukami and Ohno, 2017; Garello et al., 2014; Prenat et al., 2016).

Other consequences of the identical read and write current paths contribute to the limitations of the STTMRAMs (Kent and Worledge, 2015). Distributions of read and write current values need to be well separated in this case to avoid undesired writing while reading the memory. However, high writing currents go against energy efficiency. They also require thin tunnel barrier separating the recording and reference FM layers, resulting in reliability issues due to barrier damage at high writing currents. Moreover, optimizing the tunnel barrier (and other components of the STT-MRAM stack) for writing can have detrimental effect on the magnitude of the readout TMR. In contrast, the three-terminal SOT-MRAM bit cell with separate write and read paths allows for optimizing separately these two basic memory functionalities and to remove the endurance issue by not exposing the tunnel barrier to the writing current. These advantages come only at an expense of a larger area of the three-terminal SOT-MRAM cell (that is, a lower memory density) compared to the two-terminal STT-MRAM. Overall, SOT-MRAMs can find a broad utility and appear to be particularly well suited for the top of the memory hierarchy, namely for the embedded processor caches (Fukami and Ohno, 2017; Prenat et al., 2016).

The SHE-SOT model mechanism shares with the STT the basic concept of the angular momentum transfer from carrier spin-current to magnetization torque. As a consequence, the dominant component of the SHE-SOT in this picture is damping-like and takes the form (Ando et al., 2008), $\mathbf{T}=\left(\gamma J_{s}^{\mathrm{SHE}} / V\right) \mathbf{m} \times(\mathbf{m} \times \boldsymbol{\zeta})$. Here $\mathbf{m}=\mathbf{M} / M_{\mathrm{s}}$ is the unitary magnetization direction of the recording mag- net of volume $V, M_{\mathrm{s}}$ being the saturation magnetization, and $\boldsymbol{\zeta}$ is a unit vector of the in-plane spin-polarization of the out-of-plane SHE spin current. The magnitude of the injected SHE spin current into the magnet is modelled as, $J_{s}^{\mathrm{SHE}}=\eta \theta_{\mathrm{sh}} A(\hbar / 2 e) \sigma_{0} E$ where $\eta$ is the spininjection efficiency across the NM/FM interface of area $A, \theta_{\mathrm{sh}}=(2 e / \hbar) \sigma_{\mathrm{sh}} / \sigma_{0}$ is the spin-Hall angle in the NM material of spin-Hall conductivity $\sigma_{\text {sh }}$ and electrical conductivity $\sigma_{0}$, and $\mathbf{E} \perp \boldsymbol{\zeta}$ is the applied in-plane electric field. The SHE-SOT, being damping-like, directly competes with the damping term in the Landau-LifshitzGilbert (LLG) equation of magnetization dynamics. This is favorable because the critical current for switching does not have to reach the value necessary for overcoming the magnetic anisotropy barrier that separates spin-up and spin-down memory states. For a damping-like torque, the critical current has to overcome the magnetic anisotropy barrier multiplied by the Gilbert damping factor, the latter being typically $\ll 1$ (Ralph and Stiles, 2008).

Another common favorable feature of the SHE-SOT and STT is that the switching condition is given by the applied current density and not the absolute current, which makes the mechanism scalable and, therefore, suitable for high-density memories. In contrast, for the traditional writing method by the current-induced Oersted magnetic field, the switching condition is determined by the absolute current. This, combined with the non-locality of the magnetic field and with the fact that the corresponding field-torque has to overcome the full magnetic barrier (not reduced by the damping factor), made the Oersted switching used in the first generation MRAMs not scalable (Chappert et al., 2007).

The iSGE-SOT, as depicted in Fig. 1(c) and more microscopically explained in Fig. 2(a), resembles at first glance a mechanism in which the applied current generates a field rather than a damping-like, angular momentum transfer torque. From that perspective, it rather appears as a counterpart of the Oersted magnetic field. This would imply larger critical currents for switching by the field-like iSGE-SOT than by the damping-like SHESOT. On the other hand, the field-like iSGE-SOT shares with the SHE-SOT the other important features for the scalability, which is the switching condition determined by the current density rather than current, and the locality of the torque acting only in the cell excited by the writing current.

In the iSGE mechanism in a NM/FM bilayer, the carrier spin polarization and the corresponding field acting on the magnetization form directly at the inversionasymmetric interface. The damping-like SHE-SOT, on the other hand, has been primarily viewed as a consequence of the spin current pumped from the bulk of the NM material (which can be centrosymmetric) to the FM where it transfers its angular momentum to the magnetization. In the SHE, however, the spin current can also yield spin accumulation at the edges of the NM material 


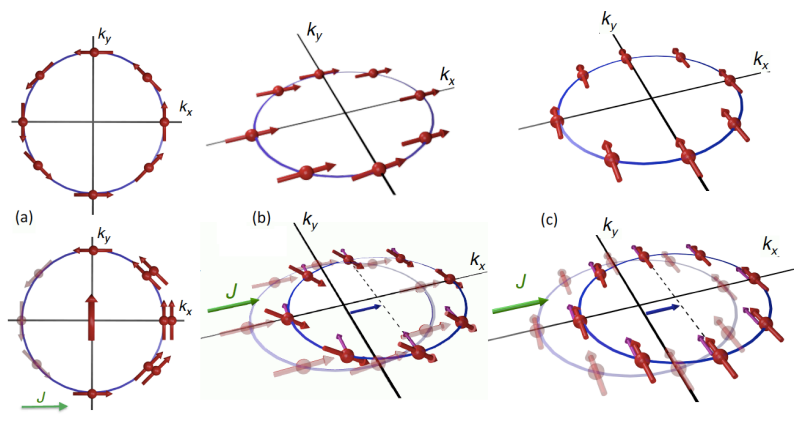

FIG. 2 (Color online) (a) Top panel: Rashba spin-texture for one of the chiral states in equilibrium with zero net spindensity. Bottom panel: Non-equilibrium redistribution of eigenstates in an applied electric field resulting in a nonzero spin-density due to broken inversion symmetry of the spin-texture. When combined with the exchange coupling of the carrier spin-density to magnetization, this mechanism corresponds to the extrinsic (Boltzmann transport), field-like iSGE-SOT. (b) Top panel: A model equilibrium spin texture in a 2D Rashba spin-orbit coupled system with an additional time-reversal symmetry breaking exchange field of a strength much larger than the spin-orbit field. In equilibrium, all spins in this case align approximately with the $x$-direction of the exchange field (magnetization). Bottom panel: In the presence of an electrical current along the $x$-direction the Fermi surface (circle) is displaced along the same direction. When moving in momentum space, electrons experience an additional spin-orbit field (purple arrows). In reaction to this non-equilibrium current induced field, spins tilt and generate a uniform, non-equilibrium out-of-plane spin-polarization. (c) Top panel: Same as in (b) for $y$-direction of the exchange field. Bottom panel: Same as in (b) but now with the current induced spin-orbit field align with the exchange field, resulting in zero tilt of the carrier spins. (b) and (c) illustrate the intrinsic (Berry curvature) damping-like iSGE-SOT. Adapted from Kurebayashi et al., 2014.

where the inversion-symmetry is broken. This implies an alternative picture of the SHE-SOT caused by the spin accumulation at the NM/FM interface. Correspondingly, the SHE can be also expected to contribute to the fieldlike SOT. Vice versa, as illustrated in Figs. 2(b,c), the iSGE mechanism can yield not only field-like but also damping-like SOT terms (Kurebayashi et al., 2014; Miron et al., 2011a). While the original iSGE models consider the effect of a uniform spin-polarization on the magnetization dynamics, additional torques arise in models where the spin accumulation generated at the interface is allowed to diffuse away from the interface (Amin and Stiles, 2016a,b; Haney et al., 2013b; Manchon, 2012).

Formally, the SOT has two orthogonal components (the third component parallel to $\mathbf{m}$ is ineffective) that can be written as

$$
\mathbf{T}=\tau_{\mathrm{FL}} \mathbf{m} \times \boldsymbol{\zeta}+\tau_{\mathrm{DL}} \mathbf{m} \times(\mathbf{m} \times \boldsymbol{\zeta}),
$$

where the vector $\zeta$ is independent of the magnetization vector $\mathbf{m}$. If $\tau_{\mathrm{FL}}$ and $\tau_{\mathrm{DL}}$ were independent of $\mathbf{m}$, one could associate the former with the field-like torque and the latter with the damping-like torque. As mentioned above, even in this idealized scenario, an experimental decomposition into $\mathbf{T}_{\mathrm{FL}}$ and $\mathbf{T}_{\mathrm{DL}}$ would not allow to disentangle the microscopic iSGE and SHE mechanisms of the SOT. Moreover, the factors $\tau_{\mathrm{FL}}$ and $\tau_{\mathrm{DL}}$ can depend on the angle of $\mathbf{m}$ (Garello et al., 2013). This makes not only the microscopic analysis but also the phenomenological LLG description of the SOT more complex.

In general, SOT can be directly linked to the applied electric field $\mathbf{E}$ by a linear-response expression, $\mathbf{T}=\chi_{T} \mathbf{E}$ (Freimuth et al., 2014b). Alternatively, SOT can be written as $\mathbf{T}=\mathbf{M} \times \mathbf{B}$, where $\mathbf{B} \approx-\Delta \mathbf{S} / M_{\mathrm{s}}$ is an effective current-induced spin-orbit field, $\Delta$ is the exchange coupling between carrier spins and magnetic moments, and $\mathbf{S}=\chi_{S} \mathbf{E}$ is the current-induced carrier spin-polarization expressed again in the linear response. The different torque terms in the LLG equation are obtained from the expansion, $\chi_{S, i j}=\chi_{S, i j}^{(0)}+\chi_{S, i j, k}^{(1)} m_{k}+\chi_{S, i j, k l}^{(2)} m_{k} m_{l}+\cdots$, where $m_{i}$ are the components of the magnetization unit vector. Here the response function coefficients for each order in $\mathbf{m}$ are independent of $\mathbf{m}$ and their matrix form reflects the underlying crystal symmetry of the considered material or structure (Hals and Brataas, 2013; Wimmer et al., 2016; Železný et al., 2017). For example, the field-like SOT corresponds to the 0th order term while the damping-like SOT term appears in the 1st order of the expansion of $\chi_{S}$.

Note that an analogous expansion can be written for $\chi_{T}$ and that the approaches using $\chi_{T}$ or $\chi_{S}$ expansion are in principle equivalent. Using $\chi_{S}$ appeals to the two-step physical picture of the SOT in which, first, the applied current polarizes the carriers (which can also appear in NM systems) and, second, the non-equilibrium carrier spins generate the torque on magnetic moments via exchange coupling. When considering $\chi_{T}$, the physical intuition based on SHE, iSGE or other non-equilibrium spin-polarization phenomena may be less apparent but the experimentally measured quantity which is the SOT is accessed directly. As a result, when evaluating the expansion coefficients using microscopic theories of $\chi_{T}$ or $\chi_{S}$, the corresponding torques may be somewhat different on the quantitative level, depending on the detailed implementation of the electronic structure and transport theory. Here using $\chi_{T}$ always represents the more rigorous approach.

In the Kubo linear response formalism, the microscopic expression for $\chi_{S}\left(\right.$ or $\left.\chi_{T}\right)$ can be split into the intraband contribution (Boltzmann theory) and the interband term (Garate and MacDonald, 2009). The former one scales with conductivity, i.e., diverges in the absence of disorder, and contributes to the field-like SOT [see Fig. 2(a)] (Manchon and Zhang, 2008). The latter one is finite in the disorder-free intrinsic limit where it is proportional to the Berry curvature of the Bloch states in the mixed spin-momentum space (Freimuth et al., 2014a; Kurebayashi et al., 2014). The intrinsic term is analogous 
to the momentum-space Berry curvature SHE (Sinova et al., 2015) and contributes to the damping-like SOT [see Fig. 2(b,c)]. As a result, the field-like SOT tends to dominate the damping-like SOT in clean systems while the trend reverses in more disordered structures. This is an example of basic guidelines that theory can provide when analyzing SOT experiments. We emphasize, however, that other terms beyond the lowest order fieldlike and damping-like torques can also significantly contribute to the total SOT, as seen in experiments (Fan et al., 2014b; Garello et al., 2013).

Finally, we note that unlike the rigorous and systematic methods based on the response functions $\chi_{S}$ or $\chi_{T}$, considering the SHE spin current as an intermediate step between the applied electrical current and the resulting SOT is an intuitive but not rigorous approach. This is because other mechanisms beyond the model SHE picture can contribute, and because in spin-orbit coupled systems spin current is not uniquely defined, in contrast to the well-defined and directly measurable spin accumulation or torque. As a result, e.g., $\theta_{\text {sh }}$ inferred from the above expression relating it to the measured torque should not be understood in the original sense of the term "Hall angle", in particular when $\theta_{\text {sh }}$ becomes comparable or larger than 1 . It just represents an effective experimental parameter providing a simple, and therefore rather vague, characterization of the charge to spin conversion efficiency in a given structure. For similar reasons, the spin-current approach has not been applied for the systematic crystal and magnetization symmetry analysis of the series of SOT terms identified in experiment. From now on, to avoid unnecessary confusion we use $\xi$ to designate charge to spin conversion efficiency in general and $\theta_{\text {sh }}$ in the specific context of SHE.

\section{B. Spin-orbit torque in antiferromagnets}

For AFs, the STT or SOT phenomenology is modified by considering a current-induced spin polarization at a particular atomic site that tends to produce a torque which acts locally on the magnetic moment centered on that site (Gomonay and Loktev, 2014; Jungwirth et al., 2016; MacDonald and Tsoi, 2011; Železný et al., 2014). In analogy to FMs, the local torques acting on the $a$ th AF sublattice magnetization, $\mathbf{M}_{a}$, have a field-like component of the form $\mathbf{T}_{a}=\mathbf{M}_{a} \times \mathbf{B}_{a}$, with $\mathbf{B}_{a} \sim \boldsymbol{\zeta}_{a}$, and a damping-like component $\mathbf{T}_{a}=\mathbf{M}_{a} \times \mathbf{B}_{a}^{\prime}$, with $\mathbf{B}_{a}^{\prime} \sim \mathbf{M}_{a} \times \boldsymbol{\zeta}_{a}$, respectively. Note that in a rigorous systematic theory, these and all other torque terms acting in an AF can be again obtained from the linear response expressions in which the coefficients of the magnetizationexpansion of $\chi_{T, a}$ (or $\chi_{S, a}$ ) reflect local crystal symmetries of the $a$-th AF sublattice (Železný et al., 2017). Assuming a collinear AF, two model scenarios can be considered for the field-like and damping-like SOTs: One with $\boldsymbol{\zeta}_{1}=\boldsymbol{\zeta}_{2}=\boldsymbol{\zeta}$ and the other one with $\boldsymbol{\zeta}_{1}=-\boldsymbol{\zeta}_{2}$.

The former case corresponds, e.g., to injection of uniformly polarized carries from an external reference FM, from a NM SHE material, or to the generation of a uniform spin-polarization at a NM/AF interface by iSGE [see e.g. (Manchon, 2017)]. The field-like torque in the AF would then be driven by a uniform non-staggered effective field $\mathbf{B}_{1}=\mathbf{B}_{2} \sim \boldsymbol{\zeta}$, i.e., would be equally inefficient in switching an AF as a uniform external magnetic field acting on an AF. On the other hand, the local nonequilibrium effective field, $\mathbf{B}_{a}^{\prime} \sim \mathbf{M}_{a} \times \boldsymbol{\zeta}$, driving the damping-like torque has an opposite sign on the two spin sublattices since $\mathbf{M}_{1}=-\mathbf{M}_{2}$. The staggered nature of the effective field commensurate with the staggered equilibrium AF order makes it equally efficient in triggering magnetization dynamics as the damping-like torque in FMs.
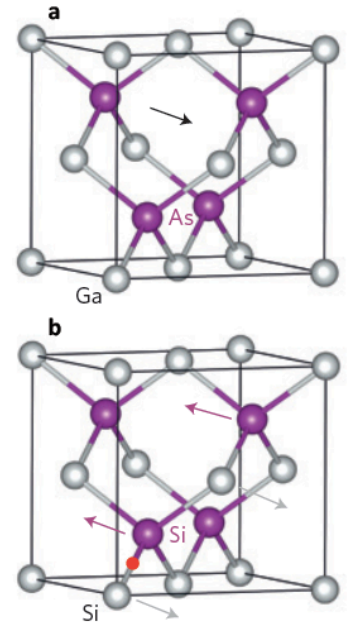

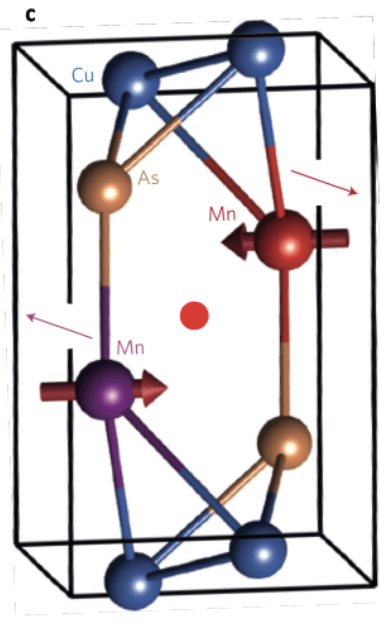

FIG. 3 (Color online) (a) Global FM-like non-equilibrium spin polarization generated by electrical current in a nonmagnet lattice with global inversion-asymmetry (e.g. GaAs) due to the iSGE. (b) Local AF-like non-equilibrium spin polarization in a non-magnet lattice with local inversionasymmetry (e.g. Si) due to the iSGE. Red dot shows the inversion-symmetry center of the Si lattice. The two Si atoms on either side of the center occupy inversion-partner lattice sites with locally asymmetric environments. In GaAs lattice, the inversion-symmetry center is absent since the two inversion-partner sites in the unit cell are occupied by different atoms. (c) Local staggered non-equilibrium spin-polarization inducing a local staggered effective field in an AF lattice with local inversion-asymmetry (e.g. CuMnAs). Thin arrows represent the current-induced staggered effective field and thick arrows the AF moments. Adapted from Jungwirth et al., 2016.

The microscopic realization of the second scenario in which $\boldsymbol{\zeta}_{1}=-\boldsymbol{\zeta}_{2}$ is illustrated in Fig. 3 (Ciccarelli et al., 2016; Jungwirth et al., 2016; Železný et al., 2014). It is the staggered counterpart of the uniform iSGE spinpolarization discussed above. As mentioned in the previous section, an electrical current driven through a crystal 
with a non-centrosymmetric unit cell, e.g. zinc-blende GaAs, generates a net non-equilibrium spin-polarization [Fig. 3(a)]. The related diamond lattice of e.g. Si, shown in Fig. 3(b), has still two atoms in the unit cell with locally non-centrosymmetric environments. The two atoms sitting on the inversion partner lattice sites are, however, identical which makes the diamond lattice unit cell globally centro-symmetric. As a result, the diamond lattice is an example where the iSGE can generate local non-equilibrium spin polarizations with opposite sign and equal magnitude on the two inversion-partner atoms while the global polarization integrated over the whole unit cell vanishes. Here a uniform electrical current induces a non-equilibrium staggered spin polarization in the bulk crystal.

In $\mathrm{Si}$ there is no equilibrium $\mathrm{AF}$ order that could be manipulated by these local staggered non-equilibrium polarizations. However, AFs like CuMnAs shown in Fig. 3(c), share the crystal symmetry allowing for the current-induced staggered polarization whose sign alternates between the inversion-partner atoms. Moreover, one inversion-partner lattice site is occupied by the magnetic atom belonging to the first AF spin sublattice and the other inversion partner is occupied by the magnetic atom belonging to the second spin sublattice. As a result, the corresponding field-like Néel SOT can reorient AF moments with an efficiency similar to the reorientation of FM moments by an applied uniform field. This scenario has been confirmed experimentally in CuMnAs and $\mathrm{Mn}_{2} \mathrm{Au}$ memory devices (Bodnar et al., 2017; Meinert et al., 2017; Wadley et al., 2016).

\section{Topology and spin-orbit torque}

In Fig. 2(a) we illustrated a microscopic picture of the current-induced spin polarization on a cartoon assuming a single-band two-dimensional (2D) Fermi surface with spins locked in the orthogonal direction to the momentum due to a strong spin-orbit coupling. In real semiconductor materials where the iSGE and SHE were initially discovered, and even more in metal structures, multiple bands cross the Fermi level and their respective contributions to the current-induced spin-polarization tend to compensate each other. Also the spin-textures are more complex, which can further reduce the net effect.

From this perspective, topological insulators (Hasan and Kane, 2010; Pesin and MacDonald, 2012b) are regarded as optimal materials for the SOT. The surface states of a three-dimensional (3D) topological insulator form a Dirac cone with a single Fermi surface and a helical locking of the relative orientations of the spin and the momentum [see Fig. 4(a)] corresponds precisely to the model of Fig. 2(a). Indeed, SOT-FMR measurements in a metal FM interfaced with a topological insulator showed an exceptionally large spin conversion efficiency

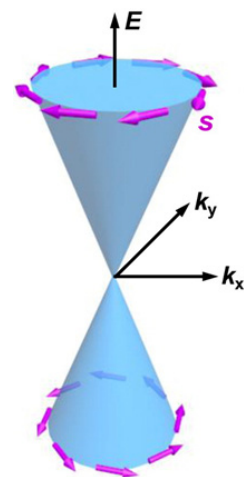

(a)

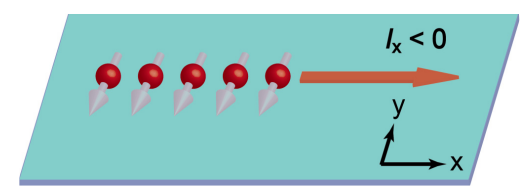

(b)

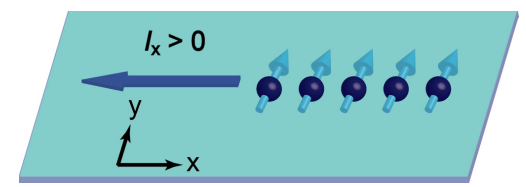

(c)
FIG. 4 (Color online) Charge current-induced surface spin polarization in topological insulator. (a) Schematic illustration of the spin-momentum locked helical spin texture of the surface states in topological insulator: clockwise spin texture above the Dirac point while anticlockwise spin texture below the Dirac point. (b) Schematic of surface spin polarization for a charge current flowing along $-x$ direction (i.e., $I_{x}<0$ ). (c) Schematic of surface spin polarization for a charge current flowing along $x$ direction (i.e., $I_{x}>0$ ). From Fan and Wang, 2016.

$\xi$ (Mellnik et al., 2014). However, compared to common NMs, the increase of $\xi$ in the studied topological insulator turned out to be primarily due to its decreased electrical conductivity while the inferred effective spin-Hall conductivity was similar to the NMs.

Interfacing a highly resistive topological insulator with a low resistive metal FM has also a practical disadvantage that most of the applied electrical current is shunted through the metallic magnet and does not contribute to the generation of the spin polarization at the topological insulator surface. A possible remedy is in using an insulating magnet. An example is a study of highly efficient magnetization switching at cryogenic temperatures in a topological-insulator/magnetic-topological-insulator bilayer, in which the inferred spin conversion efficiency $\xi$ was three orders of magnitude larger than in NMs (Fan et al., 2014b; Fan and Wang, 2016).

In the above studies, Dirac quasiparticles exhibiting strong spin-momentum locking are considered to enhance the efficiency of the SOT control of magnetic moments. Vice versa, a scheme has been recently proposed for the electric control of Dirac band crossings by reorienting magnetic moments via SOT (Šmejkal et al., 2017). Instead of 2D surface states of a topological insulator, these predictions consider Dirac bands in the bulk of a topological 3D semimetal. Since Dirac bands can only exist in systems with a combined space-inversion and time-inversion $(\mathcal{P} \mathcal{T})$ symmetry, FMs are excluded. On the other hand, the combined $\mathcal{P} \mathcal{T}$-symmetry in an $\mathrm{AF}$ is equivalent to a magnetic crystal symmetry in which AF spin-sublattices occupy inversion-partner lat- 
tice sites. This in turn allows for an efficient SOT, as discussed in the previous section.

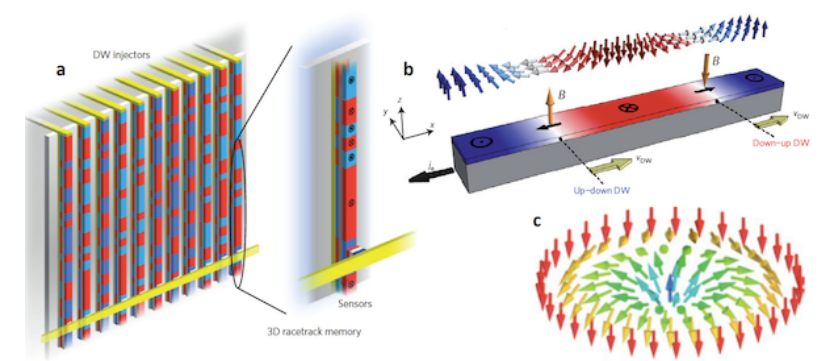

FIG. 5 (Color online) (a) Domain wall racetrack memory with red and blue regions representing areas that are oppositely magnetized. Adapted from Parkin and Yang, 2015. Illustration of left-handed chiral Néel DWs in a NM/FM bilayer. The effective field $B$ of the damping-like SOT moves adjacent up-down and down-up domains (with velocity $v_{D W}$ ) in the same direction. Adapted from Emori et al., 2013. (c) Skyrmions in a 2D FM with uniaxial magnetic anisotropy along the vertical axis. Magnetization is pointing down on the edges and pointing up in the centre. Moving along a diameter, the magnetization rotates by $2 \pi$ around an axis perpendicular to the diameter due to the DMI. Adapted from Fert et al., 2013.

Chiral spin-textures in the momentum-space of topological insulators or semimetals have their realspace counterparts in chiral domain walls (DWs) and skyrmions. These non-uniform magnetization objects are the basis of the racetrack memory concept illustrated in Fig. 5 (Fert et al., 2013; Parkin and Yang, 2015). A domain-wall racetrack memory consists of a series of alternating up and down magnetization domains that can be synchronously shifted along the corresponding multibit track and by this sequentially read by a single magnetoresistive sensor [see Fig. 5(a)].

An applied uniform (easy-axis) magnetic field cannot be used to operate the racetrack since it favors one of the two types of domains and thus pushes neighboring DWs in opposite directions. Initially, this problem was resolved by replacing magnetic field with the STT that is induced by an in-plane current driven along the racetrack (Parkin et al., 2008). The physics is analogous to STT switching by a vertical current in a FM bilayer MRAM stack where the preferred magnetization direction is controlled by the direction of the applied spin current. In the racetrack, one direction of the applied electrical current moves electrons from, say, the up-domain to the downdomain at one DW, and from down-domain to the updomain at the neighboring DW. As a result, the sense of the spin current is opposite at the two DWs. It implies that, say, the up-domain is preferred at the first DW while the down domain is preferred at the second DW and the two DWs then move in the same direction.

At first sight, SOT in a racetrack fabricated from a NM/FM bilayer cannot be used to synchronously move multiple DWs along the track. For example, a field-like SOT due to the iSGE would act as a uniform magnetic field. Also the damping-like SOT due to the SHE seems unfavorable as it is driven by a uniform vertical spin current. This makes the SHE-SOT fundamentally distinct in the DW racetrack geometry from the STT mechanism that exploits the repolarization of the in-plane spin current when carriers enter successive domains.

Remarkably, theory and experiment have shown that the damping-like SOT can also move neighboring DWs in the same direction, provided that the walls are of Néel type and have the same spin chirality (Emori et al., 2013; Ryu et al., 2013; Thiaville et al., 2012). Chiral Néel DWs are stabilized by the Dzyaloshinskii-Moriya interaction (DMI) which relies on the interfacial spin-orbit coupling and broken inversion symmetry, similarly to the SOT. In this chiral case, the effective field driving the dampinglike SHE-SOT in the DW is oriented along the easy axis in a direction that alternates from one DW to the next so that current drives them in the same direction [see Fig. 5(b)]. Moreover, in analogy to switching in MRAMs, the racetrack SOT can be more efficient than STT, resulting in higher current-induced DW velocities (Miron et al., 2011b; Yang et al., 2015).

In an alternative racetrack memory concept, the onedimensional (1D) chiral DWs are replaced with the skyrmion topological 2D chiral textures [see Fig. 5(c)] (Fert et al., 2013). Compared to the DWs, critical currents for driving skyrmions can be orders of magnitude lower, and the SOT is again expected to provide higher efficiency than the STT (Jonietz et al., 2010; Sampaio et al., 2013). Skyrmions also have the advantage that they behave as point-like particles and are in principle less sensitive to the boundaries and pinning to boundary defects as compare to DWs.

\section{Inverse effect of the spin-orbit torque}

The Onsager reciprocity relations imply that there is an inverse phenomenon to the SOT, which we call the spin-orbit charge pumping (Hals et al., 2010). The underlying physics of the spin-orbit charge pumping generated from magnetization dynamics is in the direct conversion of magnons into charge currents via spin-orbit coupling, as illustrated on Fig. 6. This effect evolves from the spin pumping predicted by Brataas et al. (2002); Tserkovnyak et al. (2002b) when SOC is included, either in the bulk of the nonmagnetic metal or at the interface. Thus, any external force that drives magnetization precession can generate spin-orbit charge pumping. Similarly to the SOT, two model microscopic mechanisms can be considered for the spin-orbit charge pumping: one due to the inverse effect of the iSGE (Ciccarelli et al., 2014; Rojas-Sánchez et al., 2013) and the other one due to the inverse SHE (Saitoh et al., 2006). Together with the non-local de- 
tection in a lateral structure (Valenzuela and Tinkham, 2006), the spin-orbit charge pumping across the NM/FM interface provided the first experimental demonstration of the inverse SHE (Saitoh et al., 2006). Since then it has evolved into one of the most common tools for electrical detection of magnetization dynamics.

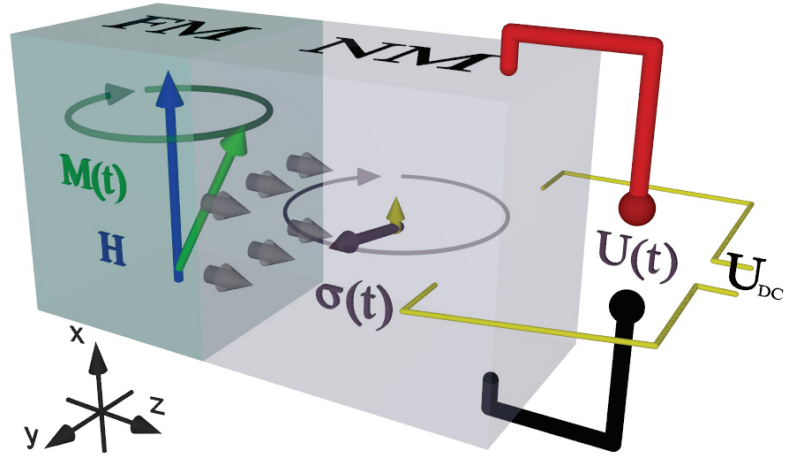

FIG. 6 (Color online) A spin current is generated by spinpumping at the NM/FM interface (grey arrows). The time dependent spin polarization $\sigma(t)$ of this current (indicated as a dark grey arrow) rotates almost entirely in the $y-z$ plane. The small time averaged dc component (yellow arrow) appears along the $x$ axis. Both components lead to charge currents in NM and can be converted into ac and dc voltages, $U(t)$ and $U_{\mathrm{DC}}$, by placing probes along the $x$ and $y$ direction, respectively. From Wei et al., 2014.

\section{THEORY OF SPIN-ORBIT TORQUES}

In this section we review the progress that has been made towards the theoretical understanding of SOTs in both layered heterostructures and bulk materials. The most general treatment of the SOT that has been considered so far is using the (spin-)density functional theory (DFT). Within DFT, the system is de- scribed by a noninteracting Hamiltonian

$$
\hat{H}=\hat{\mathcal{K}}+\hat{V}_{\mathrm{eff}}(\mathbf{r})+\hat{\boldsymbol{\sigma}} \cdot \boldsymbol{\Omega}_{\mathrm{xc}}(\mathbf{r})+\hat{H}_{\mathrm{so}}
$$

where $\hat{\mathcal{K}}$ is the kinetic energy, $\hat{V}_{\text {eff }}$ is the effective crystal potential, $\boldsymbol{\Omega}_{\mathrm{xc}}$ is the exchange-correlation field, and $\hat{H}_{\mathrm{so}}$ is the spin-orbit coupling. Assuming this form of the Hamiltonian, the torque on magnetization at point $\mathbf{r}$ is given by (Haney et al., 2008; Manchon and Zhang, 2011)

$$
\mathbf{T}(\mathbf{r})=-\mathbf{S}(\mathbf{r}) \times \boldsymbol{\Omega}_{\mathrm{xc}}(\mathbf{r}),
$$

where $\mathbf{S}=(1 / V)\langle\hat{\boldsymbol{\sigma}}\rangle$ is current induced spin density, and $V$ is the volume of the unit cell. This equation is valid for the STT as well as for the SOT. When spin-orbit coupling is neglected, the torque can be equivalently expressed as a divergence of a spin current (Ralph and Stiles, 2008)

$$
T_{i}(\mathbf{r})=-\nabla \cdot \mathcal{J}_{\mathrm{s}}^{i},
$$

where $\mathcal{J}_{\mathrm{s}}^{i}=(\hbar / 4)\left\langle\left\{\hat{\sigma}_{i}, \hat{\mathbf{v}}\right\}\right\rangle$ is the $i$-th spin component of the spin current. In the absence of spin-orbit coupling, the torque is thus directly given by the absorption of the spin current. However, when spin-orbit coupling is not neglected, the spin angular momentum is not a conserved quantity and the spin current in Eq. (4) is then not uniquely defined, while Eq. (3) remains valid.

The total torque is obtained by integrating Eq. (3) over the whole unit cell, and a local torque is obtained by integrating over a particular magnetic atom. This torque can then be inserted into LLG equations to evaluate the magnetic dynamics induced by the SOT. When using this approach it is necessary to ensure that the dynamics of the non-equilibrium carrier spins is much faster than the dynamics of magnetic moments arising from equilibrium electrons; otherwise the dynamics of the two could not be separated. This is well justified in FMs whose magnetization dynamics lies in the $\mathrm{GHz}$ range but it could become an issue when discussing AFs whose dynamics can reach several THz. We also note that Eq. (3) assumes that Eq. (2) accurately describes the electronic system. This is reasonable for most materials of interest, namely metals, but fails in strongly correlated systems. In these systems, more sophisticated many-body approaches are necessary. So far SOTs have been studied only using noninteracting model (free electron or k.p) Hamiltonians or Kohn-Sham Hamiltonians originating from DFT.

At weak applied electric fields, the SOT is well described by linear response theory, $\mathbf{T}=\chi_{T} \mathbf{E}$, where the response tensor $\chi_{T}$ can be calculated using Eq. (3). Equivalently, the torque can be rewritten as, $\mathbf{T}=\mathbf{M} \times \mathbf{B}$, with the effective field obtained from the linear response expression, $\mathbf{B}=\chi_{B} \mathbf{E}$. In many calculations of the SOT, especially those based on model Hamiltonians, an approximation is used in which the effective magnetic field is made directly proportional to the current-induced spinpolarization, $\mathbf{B} \approx-\Delta \mathbf{S} / M_{\mathrm{S}}$ Here $\Delta$ is an exchange coupling energy corresponding to exchange between the carrier spins and magnetic moments and $\mathbf{S}$ is again evaluated using linear response, $\mathbf{S}=\chi_{S} \mathbf{E}$.

As discussed in Section II, the origin of the SOT in the bilayer systems is often attributed to two different effects, the SHE and the iSGE, where the SHE-SOT is assumed to originate from the absorption of a spin current generated in the NM [see Fig. 7(a)] and the iSGE-SOT is due to spin-polarization generated locally in the FM or at the interface [see Fig. 7(b)]. Equation (3) shows however that the torque always originates from a current-induced spin-polarization. Thus the SHE-SOT can be more fundamentally understood not in terms of the absorption of a spin current but in terms of a spin accumulation induced by the spin Hall current. Consequently, both contributions can be treated on the same footing and there is no clear way how to theoretically separate them.

Still, it is intuitively appealing to separate the total torque into a contribution associated with the absorption 
of a spin current, as given by Eq. 4, and a contribution due to a locally generated spin-polarization, described by Eq. 3. One could then attribute the former contribution to SHE and the latter one to iSGE. However, such an approach has several drawbacks. First, spin currents are not necessarily due to the bulk SHE only as substantial contributions can also come from the interface with the FM (Amin and Stiles, 2016a,b; Kim et al., 2017b; Wang et al., 2016a). Second, even in bulk non-centrosymmetric materials where SOT is considered of purely iSGE origin, local spin currents within the unit cell can contribute to the torque. Third, in a slab geometry, interface and bulk are not well defined notions, and the terminology of what should be referred to as iSGE or SHE becomes unclear (Freimuth et al., 2014b). Conventionally, iSGE refers to spin-polarization generated internally in the material. However, even the spin accumulation induced by SHE in the bilayers could be referred to as iSGE, since it is also a spin-polarization induced by a charge current. In conclusion, although models based on bulk SHE or iSGE due to interfacial Rashba spin-orbit coupling can be useful to explain some aspects of the experiments, in real systems there is not much point in trying to rigorously parse the torque into these two contributions.

In many experimental studies, the origin of the SOT is analyzed in terms of its symmetries. The dampinglike (DL) torque $\mathbf{T}_{\mathrm{DL}}$ is often referred to as spin Hall or Slonczewski torque, and the field-like (FL) torque $\mathbf{T}_{\mathrm{FL}}$ as spin-orbit or Rashba torque. This is based primarily on the assumption that any torques associated with transfer and absorption of spin-angular momentum would have dissipative-like character and the one arising from the iSGE would be primarily FL. This is however not the case, as interband transitions, spin relaxation and size effects significantly complicate the SOT scenario. Hence, symmetry considerations alone cannot disentangle directly the two contributions. However, symmetry analysis remains a powerful tool. SOTs obey Neumann's principle and must be invariant under the symmetry operations of the material system. This can restrict significantly the forms of the response coefficients, and aids the formulation of the proper phenomenological description of the SOTs, reflecting the underlying crystal symmetry of the considered material or structure (Hals and Brataas, 2013; Wimmer et al., 2016; Železný et al., 2017).

This section is organized as follows. We review the linear response formalism commonly used for microscopic calculations of the SOT in Subsection III.A, and the general symmetry properties of SOT are then discussed in Subsection III.B. Because of the great challenge of incorporating the full complexity of the bilayer systems at once, almost all theoretical studies have been focused either on iSGE in model systems or on the SHE mechanism only, with a handful of them attempting a comprehensive modeling. In Subsection III.C we review calculations of the SOT in bilayer systems based on the SHE mechanism. In Subsection III.D we review calculations of the SOT in bulk systems which includes the 2D Rashba model and 3D non-centrosymmetric materials. Microscopic calculations carried out using DFT calculations for bilayer structures are presented in Subsection III.E. In Subsection III.F we review calculations of the SOT in bulk AFs, and a discussion of SOTs in topological insulators and other systems is presented in Subsections III.G and III.H, respectively.

(a)

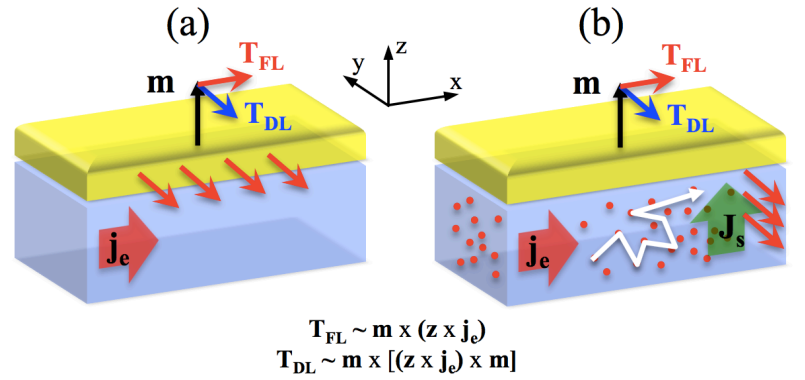

FIG. 7 (Color online) Two main model spin-charge conversion mechanisms at the NM/FM interface: (a) iSGE and (b) SHE. Both mechanisms produce damping-like and field-like torques.

\section{A. Kubo linear response: intraband versus interband transitions}

From a microscopic linear response perspective, basic quantum mechanics states that the statistical average of an operator $\hat{\mathcal{O}}$ reads $\mathcal{O}=\sum_{n, \mathbf{k}}\langle n, \mathbf{k}|\hat{\mathcal{O}}| n, \mathbf{k}\rangle f_{n, \mathbf{k}}$, where $f_{n, \mathbf{k}}$ is the carrier distribution function and $|n, \mathbf{k}\rangle$ is the quantum eigenstate of the system. Under a small perturbation, such as an external electric field, both the distribution function $f_{n, \mathbf{k}}$ and the eigenstates $|n, \mathbf{k}\rangle$ are modified, giving rise to different nonequilibrium contributions to the observable $\mathcal{O}$ as consistently modeled by quantum field theory (Mahan, 2000; Rammer and Smith, 1986). Within the constant relaxation time approximation, the distribution function and eigenstates become

$$
\begin{aligned}
& f_{n, \mathbf{k}} \rightarrow f_{n, \mathbf{k}}^{0}-\tau\langle n, \mathbf{k}|e \mathbf{E} \cdot \hat{\mathbf{v}}| n, \mathbf{k}\rangle \partial_{\epsilon} f_{n, \mathbf{k}}^{0} \\
& |n, \mathbf{k}\rangle \rightarrow|n, \mathbf{k}\rangle_{0}-\sum_{n^{\prime}} \frac{\left\langle n^{\prime}, \mathbf{k}|e \mathbf{E} \cdot \hat{\mathbf{r}}| n, \mathbf{k}\right\rangle_{0}}{\epsilon_{n, \mathbf{k}}-\epsilon_{n^{\prime}, \mathbf{k}}}\left|n^{\prime}, \mathbf{k}\right\rangle_{0}
\end{aligned}
$$

where $f_{n, \mathbf{k}}^{0}$ is the Fermi-Dirac distribution, $\mathbf{E}$ is the electric field, $\hat{\mathbf{v}}$ and $\hat{\mathbf{r}}$ are the velocity and position operators, and $\epsilon_{n, \mathbf{k}}$ is the eigenenergy associated with the unperturbed eigenstate $|n, \mathbf{k}\rangle_{0}$. As a result, within the linear response approximation and to the lowest order in relax- 
ation time, $\mathcal{O}=\mathcal{O}^{\text {Intra }}+\mathcal{O}^{\text {Inter }}$, where

$$
\mathcal{O}^{\text {Intra }}=\tau \sum_{n, \mathbf{k}} \operatorname{Re}\langle n, \mathbf{k}|e \mathbf{E} \cdot \hat{\mathbf{v}}| n, \mathbf{k}\rangle\langle n, \mathbf{k}|\hat{\mathcal{O}}| n, \mathbf{k}\rangle \delta\left(\epsilon_{n, \mathbf{k}}-\epsilon_{\mathrm{F}}\right) .
$$

$$
\begin{aligned}
\mathcal{O}^{\text {Inter }}= & -\hbar \sum_{n, n^{\prime}, \mathbf{k}} \operatorname{Im}\left\langle n, \mathbf{k}|e \mathbf{E} \cdot \hat{\mathbf{v}}| n^{\prime}, \mathbf{k}\right\rangle\left\langle n^{\prime}, \mathbf{k}|\hat{\mathcal{O}}| n, \mathbf{k}\right\rangle \\
& \times \frac{\left(f_{n, \mathbf{k}}-f_{n^{\prime}, \mathbf{k}}\right)}{\left(\epsilon_{n, \mathbf{k}}-\epsilon_{n^{\prime}, \mathbf{k}}\right)^{2}} .
\end{aligned}
$$

The first contribution, Eq. (7), is proportional to the relaxation time $\sim \tau$ and only involves intraband transitions, $|n, \mathbf{k}\rangle \rightarrow|n, \mathbf{k}\rangle$. The second one, Eq. (8), is weakly dependent on disorder and sometimes called intrinsic. It only involves interband transitions $|n, \mathbf{k}\rangle \rightarrow\left|n^{\prime}, \mathbf{k}\right\rangle$. The intrinsic contribution can be related to the Berry curvature of the material that connects intrinsic transport properties to the topology of the phase space (Sinova et al., 2015; Xiao et al., 2010). Eqs. (7),(8) are valid only under the assumption of a constant and large relaxation time. More generally, the linear response can be expressed in terms of the Kubo-Bastin formula (Freimuth et al., 2014b; Wimmer et al., 2016)

$$
\begin{aligned}
& \mathcal{O}=\mathcal{O}^{I(a)}+\mathcal{O}^{I(b)}+\mathcal{O}^{I I} \\
& \mathcal{O}^{I(a)}=\frac{e}{h} \int_{-\infty}^{\infty} d \varepsilon \partial_{\varepsilon} f_{\varepsilon}^{0} \operatorname{Tr}\left\langle\hat{\mathcal{O}} \hat{G}_{\varepsilon}^{R}(\mathbf{E} \cdot \hat{\mathbf{v}}) \hat{G}_{\varepsilon}^{A}\right\rangle_{c} \\
& \mathcal{O}^{I(b)}=-\frac{e}{h} \int_{-\infty}^{\infty} d \varepsilon \partial_{\varepsilon} f_{\varepsilon}^{0} \operatorname{Re} \operatorname{Tr}\left\langle\hat{\mathcal{O}} \hat{G}_{\varepsilon}^{R}(\mathbf{E} \cdot \hat{\mathbf{v}}) \hat{G}_{\varepsilon}^{R}\right\rangle_{c} \\
& \mathcal{O}^{I I}=\frac{e}{h} \int_{-\infty}^{\infty} d \varepsilon f_{\varepsilon}^{0} \operatorname{Re} \operatorname{Tr} \\
&\left\langle\hat{\mathcal{O}} \hat{G}_{\varepsilon}^{R}(\mathbf{E} \cdot \hat{\mathbf{v}}) \partial_{\varepsilon} G_{\varepsilon}^{R}-\hat{\mathcal{O}} \partial_{\varepsilon} \hat{G}_{\varepsilon}^{R}(\mathbf{E} \cdot \hat{\mathbf{v}}) \hat{G}_{\varepsilon}^{R}\right\rangle_{c}
\end{aligned}
$$

where $\hat{G}_{\varepsilon}^{R(A)}$ denotes the retarded (advanced) Green's function respectively and $\langle\ldots\rangle_{c}$ denotes an average over disorder configurations. Tr is the trace over spin, momentum and orbital spaces. For concreteness, the operator $\hat{\mathcal{O}}$ is simply the spin operator $\hat{\boldsymbol{\sigma}}$ or the spin current operator $\mathcal{J}_{\mathrm{s}}^{i}$ defined above. This formula is often simplified by assuming that the only effect of disorder is to induce a constant energy broadening $\Gamma=\hbar / 2 \tau$, such that $\hat{G}_{\varepsilon}^{R(A)}=\hbar(\varepsilon-\hat{H} \pm i \Gamma)^{-1}$. In the limit of large relaxation time, $\Gamma \rightarrow 0$ and the Kubo-Bastin formula reduces to Eqs. (7) and (8). Notice that extrinsic contributions to SHE (side-jump and skew scattering) are overlooked within this approximation (Sinova et al., 2015). Thus for a complete treatment, more sophisticated approaches are necessary.

\section{B. Symmetry of spin-orbit torques}

As mentioned above, the torque can always be rewritten in terms of an effective magnetic field $\mathbf{B}, \mathbf{T}=\mathbf{M} \times \mathbf{B}$.
The symmetry of the SOT can be studied either in terms of the linear response tensor $\chi_{T}$ or, equivalently, in terms of $\chi_{B}$. Here we focus on the effective field since its symmetry relations are simpler. In terms of symmetry, the effective magnetic field is equivalent to the iSGE, i.e., the tensors $\chi_{B}$ and $\chi_{S}$ have the same form (although not necessarily proportional, as often assumed in model calculations). To understand the symmetry properties of the SOT, it is convenient to parse the effective field into two parts, even and odd under time-reversal (or, equivalently, under the reversal of all magnetic moments). This is similar to the case of conductivity in magnetic systems (Grimmer, 1993). However, unlike for conductivity, the even and odd parts do not correspond to the symmetric and anti-symmetric parts of the effective field tensor. Thus a separate linear response tensor has to be assigned to each part,

$$
\begin{aligned}
\mathbf{B}_{\text {eff }}^{\text {even }} & =\chi_{B}^{\text {even }} \mathbf{E}, \\
\mathbf{B}_{\text {eff }}^{\text {odd }} & =\chi_{B}^{\text {odd }} \mathbf{E} .
\end{aligned}
$$

The same parsing can also be done for the torque. We note that the odd part of the torque corresponds to the even part of the effective field and vice versa. Noticeably, the odd and even parts have very different properties and correspond to different contributions of the Kubo formula: the intraband formula, Eq. (7), corresponds to the even field, whereas the interband formula, Eq. (8), corresponds to the odd field. Similar separation can be done for the full Kubo-Bastin formula (Freimuth et al., 2014b). Furthermore, such a separation is also commonly done for experimental measurements of SOT (see Sections IV and VI). Since the following applies equally to $\chi_{B}$ and $\chi_{S}$ we denote the tensor simply by $\chi$. Following the Neumann's principle, the tensors $\chi$ have to be invariant under all symmetry operations of the crystal. The two parts transform differently for symmetry operations that contain time-reversal symmetry. For a symmetry operation represented by a matrix $\mathcal{R}$ (Železný et al., 2017),

$$
\begin{aligned}
\chi^{\text {even }} & =\operatorname{det}(\mathcal{R}) \mathcal{R} \chi^{\text {even }} \mathcal{R}^{-1}, \\
\chi^{\text {odd }} & = \pm \operatorname{det}(\mathcal{R}) \mathcal{R} \chi^{\text {odd }} \mathcal{R}^{-1},
\end{aligned}
$$

where \pm refers to a symmetry operation with and without time-reversal, respectively, and $\operatorname{det}(\mathcal{R})$ is the determinant of $\mathcal{R}$. By considering all the symmetry operations in the magnetic point group of the given crystal, the general form of the response tensors is found from these equations. It is also possible to treat the whole tensor together without separating it into the even and odd parts, although then some information about the structure of the torque is lost. See (Wimmer et al., 2016) for a table of total $\chi_{T}$ tensors for all the magnetic point groups.

In systems with more than one magnetic atom in the unit cell, such as AFs, it is furthermore useful to study 
the symmetry of SOT on each magnetic site. Then Eqs. (15), (16) are modified as follows (Železný et al., 2017),

$$
\begin{aligned}
\chi_{a^{\prime}}^{\text {even }} & =\operatorname{det}(\mathcal{R}) \mathcal{R} \chi_{a}^{\text {even }} \mathcal{R}^{-1}, \\
\chi_{a^{\prime}}^{\text {odd }} & = \pm \operatorname{det}(\mathcal{R}) \mathcal{R} \chi_{a}^{\text {odd }} \mathcal{R}^{-1},
\end{aligned}
$$

where $a$ denotes a given site and $a^{\prime}$ is the site to which site $a$ transforms under symmetry operation $\mathcal{R}$. In this case it is necessary to consider the full magnetic space group and atomic positions of magnetic moments. The symmetry of $\chi_{a}$ is determined by symmetry operations that leave site $a$ invariant (such symmetry operations form the so-called site symmetry group), whereas the symmetry operations that transform $a$ to a different site $a^{\prime}$, relate tensor $\chi_{a}$ to tensor $\chi_{a^{\prime}}$.

A key conclusion that can be made from Eqs. (15),(16) is that there can be no net SOT (or iSGE) if the system has inversion symmetry. However, from Eqs. (15),(16) we see that even in a system with inversion symmetry there can still be a local SOT if the inversion symmetry is broken locally, i.e., there can be SOT on site $a$, if there is no inversion symmetry which would leave site $a$ invariant.

To understand the dependence of SOT on the direction of magnetic moments, it is helpful to expand the SOT in the direction of magnetic moments. For a collinear magnetic material,

$$
\chi_{i j}(\mathbf{n})=\chi_{i j}^{(0)}+\chi_{i j, k}^{(1)} n_{k}+\chi_{i j, k l}^{(2)} n_{k} n_{l}+\ldots,
$$

where $\mathbf{n}$ is the magnetic order parameter (the magnetization direction in FMs, or the Néel order parameter in AFs). The even terms in the expansion correspond to the even effective field and conversely the odd terms correspond to the odd field. The symmetry of the $n$ independent expansion tensors in Eq. (19) is determined by the symmetry group of the nonmagnetic system. For a global SOT in a FM or a local SOT in an AF the following transformation rule is found for the expansion tensors,

$$
\chi_{i j, m n \ldots}^{(i)}=\operatorname{det}(\mathcal{R})^{i-1} \mathcal{R}_{i k} \mathcal{R}_{j l}^{-T} \mathcal{R}_{m o}^{-T} \mathcal{R}_{n p}^{-T} \ldots \chi_{k l, o p \ldots}^{(i)},
$$

For the global case, the nonmagnetic point group has to be used, whereas for the local case, the nonmagnetic site symmetry group has to be used instead. Since there are only 21 nonmagnetic point groups with broken inversion symmetry, it is feasible to calculate all allowed leading terms of the expansion (19). This was done for the zeroth, first, and some second order terms in Refs. (Ciccarelli et al., 2016; Železný et al., 2017). The results for the zeroth and first order terms are given in Table I.

The lowest order even field is typically given by $\chi^{(0)}$, which corresponds to a FL torque. In some cases such a term is, however, prohibited by symmetry and the lowest order even field is second order in magnetization. This is the case of the cubic zinc-blende or half-heusler crystal
FMs, for instance (with strain, these materials have a FL torque, however). The relation between the directions of the effective magnetic field and the applied electric field can be categorized generally in three different types illustrated in Fig. 8: generalized Rashba and Dresselhaus coupling schemes, and a coupling such that the magnetic field is proportional to the electric field that we refer to as a Weyl coupling [Fig. 8(d)]. The Rashba and Dresselhaus fields are confined to a plane and only appear for electric field lying in the plane. They differ in how the effective field is changed when the electric field is rotated. In the case of standard Rashba coupling, the effective field rotates in the same direction as the electric field [Fig. 8(a)], whereas in the case of standard Dresselhaus coupling the effective field rotates in the opposite direction [Fig. 8(b)]. The generalized Rashba coupling differs from a conventional Rashba coupling in that the angle between the electric and effective field is not necessarily $90^{\circ}$ [Fig. 8(c)]. The generalized Dresselhaus coupling differs from the conventional Dresselhaus coupling in that the effective field is not necessarily parallel or perpendicular to the electric field along the crystalline axes [Fig. $8(\mathrm{~d})]$.

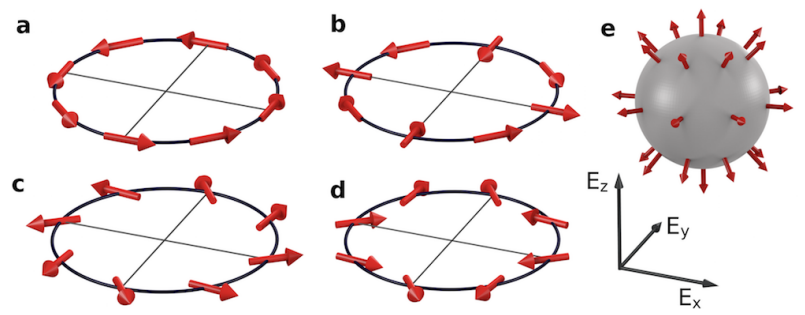

FIG. 8 (Color online) Various types of the FL torques as a function of the electric field direction. The red arrows denote the corresponding effective field direction for (a) Rashba, (b) Dresselhaus, (c) generalized Rashba, (d) generalized Dresselhaus, and (e) Weyl coupling schemes.

As seen in Tab. I, $\chi^{(1)}$ has always some non-zero components. These generate the lowest order odd field. It often has a DL character, i.e., can be written as $\mathbf{B} \sim \mathbf{m} \times \boldsymbol{\zeta}$, where $\boldsymbol{\zeta}$ is a vector independent of magnetization. However in some cases the first-order field does not have the DL form. An example of a system where no DL torque is allowed by symmetry is again cubic zinc-blende or halfheusler crystals. Even if the DL torque is allowed by symmetry there can be other first-order contributions. Magnetic dynamics induced by such torques can differ from the effect of a DL torque.

The lowest order terms frequently describe qualitative aspects of the torque both in experiments and in the theoretical calculations. The usefulness of the lowest order term is illustrated by the fact that materials with very different electronic structures but same symmetry have very similar SOTs. For instance this is the case of FM (Ga,Mn)As and NiMnSb, or systems modeled by the 
2D Rashba Hamiltonian, or AFs $\mathrm{Mn}_{2} \mathrm{Au}$ and CuMnAs, discussed in Section III.F. For an accurate quantitative description of the SOT, higher order terms can be important. These are not tabulated but can be produced by the publicly available code that was used for generating Table I (Zelezný, 2017). This code can be also used to determine the full tensors $\chi_{a}^{\text {even }}$ and $\chi_{a}^{\text {odd }}$ for a given crystal.

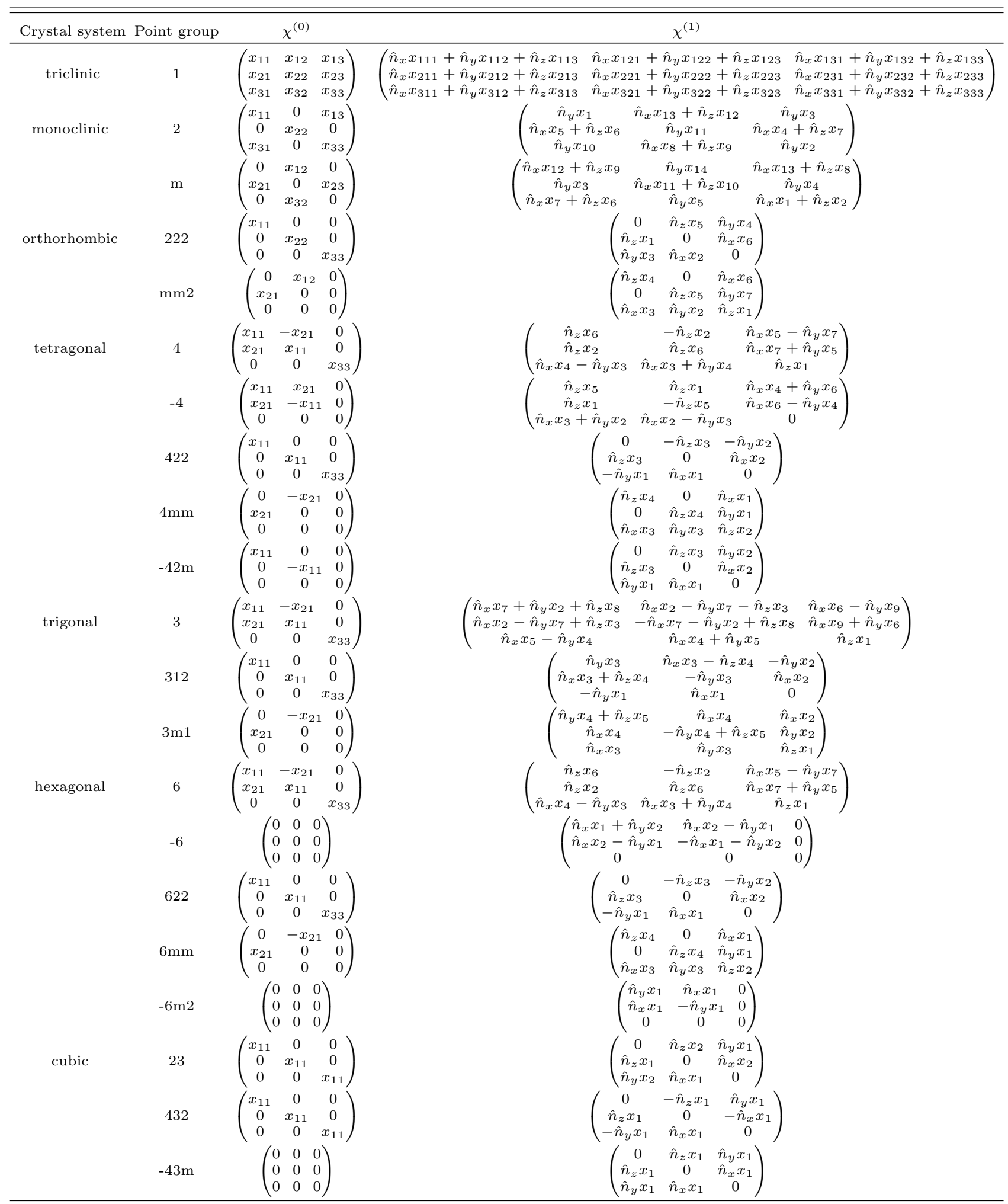


TABLE I: Zeroth and first order terms in the expansion (19) for the point groups with broken inversion symmetry. The tensors $\chi^{(1)}$ have the spin-axis direction included: $\chi_{i j}^{(1)}=\chi_{i j, k}^{(1)} \hat{n}_{k}$. The $x$ parameters can be chosen arbitrarily for each tensor. The tensors are given in cartesian coordinate systems defined in (Železný et al., 2017).

\section{Spin Hall effect spin-orbit torque}

The SHE-SOT contribution in bilayer system arises from the absorption of angular momentum coming from a SHE spin current generated outside the FM, e. g. in the proximate NM layer (Dyakonov and Perel, 1971). This is effectively the mechanism of STT where the polarizing FM in a trilayer device is replaced in this instant by the NM (Brataas et al., 2012b; Stiles and Zangwill, 2002). In analogy to the STT, the SHE-SOT mechanism in common metal structures is primarily DL in character, assuming a full absorption of the carrier spin angular momentum in the FM. Therefore in many experiments, the DL SOT is associated with the SHE, and the extracted spin Hall angle is calculated on the basis that this is the only contribution to the DL SOT component. Since this is generally not the case, the spin Hall angle values extracted from these experiments should be considered only as effective phenomenological descriptions of the SOT efficiency.

On the other hand, in many experiments a clear correlation between the magnitude and sign of bulk SHE and DL SOT is observed. We do not review here the calculations of the bulk SHE, which has been done elsewhere (Sinova et al., 2015) and focus instead on effective theoretical treatments using the bulk spin Hall angle as a phenomenological parameter.

The SHE-SOT is present in structure where the FM is adjacent to a $\mathrm{NM}$ (Pt, $\mathrm{W}, \mathrm{Ta}, \mathrm{Bi}_{2} \mathrm{Se}_{3}, \mathrm{WTe}_{2}$ etc.). To model this torque, one needs to compute the spin accumulation originating from the $\mathrm{NM}$ and diffusing into the FM. The simplest method is to solve the drift-diffusion equation in the presence of spin-orbit coupling and match the spin currents and accumulations at the boundary between the FM and the NM using, for instance, the spin mixing conductance (Amin and Stiles, 2016b; Haney et al., 2013b). The charge and spin currents in a NM with spin-orbit coupling read (Dyakonov and Perel, 1971; Shchelushkin and Brataas, 2005; Shen et al., 2015)

$$
\begin{aligned}
& e \mathbf{j}_{\mathrm{c}} / \sigma_{\mathrm{N}}=-\nabla \mu_{c}+\frac{\theta_{\mathrm{sh}}}{2} \nabla \times \boldsymbol{\mu}, \\
& e^{2} \mathcal{J}_{\mathrm{s}}^{i} / \sigma_{\mathrm{N}}=-\nabla \frac{\mu_{i}}{2}-\theta_{\mathrm{sh}} \mathbf{e}_{i} \times \nabla \mu_{c}-\frac{\theta_{\mathrm{sw}}}{2} \nabla \times\left(\mathbf{e}_{i} \times \boldsymbol{\mu}\right),
\end{aligned}
$$

where $\sigma_{\mathrm{N}}$ is the bulk conductivity, $\theta_{\mathrm{sh}}$ is the spin Hall angle and $\theta_{\text {sw }}$ is the spin swapping coefficient (Lifshits and Dyakonov, 2009) (we comment on the spin swapping term in more detail at the end of this subsection). $\mu_{c}=n / e \mathcal{N}$ and $\boldsymbol{\mu}=\mathbf{S} / e \mathcal{N}$ are the charge and spin chemical poten- tials, respectively, with $\mathcal{N}$ the Fermi density of states. Formally, the drift-diffusion approach in current-in-plane geometry is only applicable as long as the mean free path is much shorter than the layer thickness. Using the spin mixing conductance as a boundary condition, $g^{\uparrow \downarrow}$, the spin transfer arises from the absorption of the incoming transverse spin current at the interface, $\mathbf{T}=\mathcal{J}_{\mathrm{s}}$. It is composed of two components, as described in Eq. (1), which read (Haney et al., 2013b)

$$
\begin{aligned}
& e \tau_{\mathrm{DL}}=-\frac{\tilde{g}_{r}^{\uparrow \downarrow}+\left|\tilde{g}^{\uparrow \downarrow}\right|^{2}}{\left(1+\tilde{g}_{r}^{\uparrow \downarrow}\right)^{2}+\tilde{g}_{i}^{\uparrow \downarrow 2}} \theta_{\mathrm{sh}}\left(1-\cosh ^{-1} \frac{d_{\mathrm{N}}}{\lambda_{\mathrm{sf}}}\right) \sigma_{\mathrm{N}} \partial_{x} \mu_{c}, \\
& e \tau_{\mathrm{FL}}=\frac{\tilde{g}_{i}^{\uparrow \downarrow}}{\left(1+\tilde{g}_{r}^{\uparrow \downarrow}\right)^{2}+\tilde{g}_{i}^{\uparrow \downarrow}} \theta_{\mathrm{sh}}\left(1-\cosh ^{-1} \frac{d_{\mathrm{N}}}{\lambda_{\mathrm{sf}}}\right) \sigma_{\mathrm{N}} \partial_{x} \mu_{c},
\end{aligned}
$$

Here, we omitted the spin swapping term in Eq. (22), and $\boldsymbol{\zeta} \| \mathbf{z} \times \mathbf{j}_{c}$, $\mathbf{z}$ being normal to the interface. We also define $\tilde{g}^{\uparrow \downarrow}=g^{\uparrow \downarrow} \lambda_{\mathrm{sf}} /\left[\sigma_{\mathrm{N}} \tanh \left(d_{\mathrm{N}} / \lambda_{\mathrm{sf}}\right)\right]$, and $d_{\mathrm{N}}$ and $\lambda_{\mathrm{sf}}$ are the thickness and spin relaxation length of the NM. Finally, $\tilde{g}_{r}^{\uparrow \downarrow}$ and $\tilde{g}_{i}^{\uparrow \downarrow}$ refer to the real part and imaginary part of $\tilde{g}^{\uparrow \downarrow}$, respectively. In the limit of small imaginary part of the mixing conductance, $\tau_{\mathrm{DL}} \propto g_{r}^{\uparrow \downarrow}$ and $\tau_{\mathrm{FL}} \propto g_{i}^{\uparrow \downarrow}$.
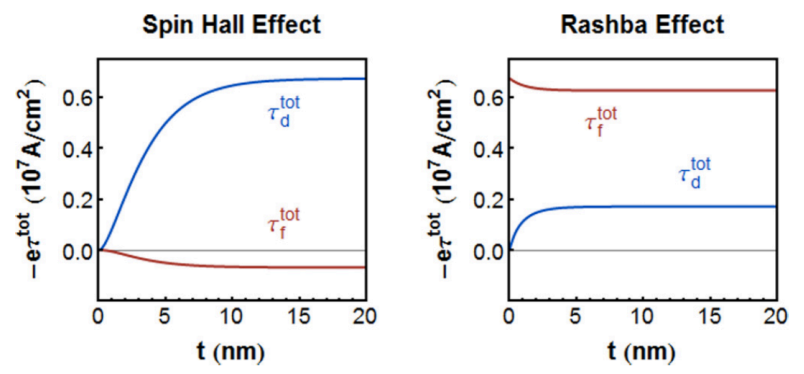

FIG. 9 (Color online) Torque components as a function of the NM thickness, in the case of SHE (left) and iSGE (right). Both mechanisms produce FL and DL components. From Amin and Stiles (2016b).

These expressions, although quite extensively used to interpret experimental data, must be handled with care as they disregard any correction emerging from semiclassical size effect and assume the simplest form of the interfacial spin mixing conductance. A more refined method to model transport in thin magnetic multilayers is to numerically solve Boltzmann equation where SHE is explicitly contained in the collision integral (Amin and Stiles, 2016b; Engel et al., 2005; Haney et al., 2013b). In this case, semiclassical size effects are properly taken into account. An example of numerical results in shown on Fig. 
9, where the torque magnitude is plotted against the NM thickness in the case of pure SHE and pure iSGE.

We end this subsection by commenting the spin swapping term in Eq. (22). It arises from the concept theoretically introduced by Lifshits and Dyakonov (2009), iluustrated in Fig. 10(a), which remains to be experimentally observed but can in principle coexist with the SHE (Saidaoui and Manchon, 2016). Spin-polarized electrons flowing through the FM layer that scatter towards the NM experience spin precession around the spin-orbit field oriented normal to the scattering plane $\left(\sim \mathbf{k} \times \mathbf{k}^{\prime} \propto \mathbf{z} \times \mathbf{j}_{\mathrm{c}}\right)$, which produces a new spin current that is spin polarized along the direction $\mathbf{m} \times\left(\mathbf{z} \times \mathbf{j}_{\mathrm{c}}\right)$. Once absorbed into the FM, this spin swapping spin current induces a FL SOT. As long as the thickness of the NM is larger than the mean free path, the system is in the diffusive regime and is well described by the SHE theory discussed above. However, when the thickness of the NM becomes comparable to the mean free path [Fig. 10(a)], spin swapping becomes increasingly large and generates an additional FL SOT [Fig. 10(b)].
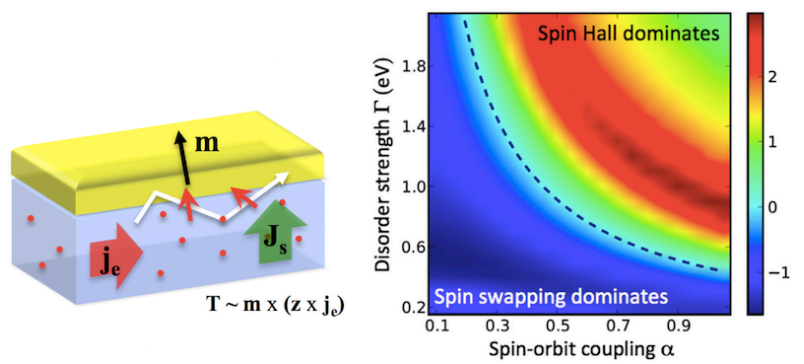

FIG. 10 (a) (Color online) Schematics of spin swapping effect in a bilayer composed of a NM (blue) and a FM (yellow) with magnetization $\mathbf{m}$. (b) Ratio between the magnitude of the FL and DL torque $\tau_{\mathrm{DL}} / \tau_{\mathrm{FL}}$ as a function of disorder strength and spin-orbit coupling. The ratio is given in logarithmic scale and the dashed line indicates $\tau_{\mathrm{DL}}=\tau_{\mathrm{FL}}$. From Saidaoui and Manchon (2016).

\section{Spin-orbit torque in two- and three-dimensional bulk systems}

In this section we review calculations of the SOT in $2 \mathrm{D}$ and $3 \mathrm{D}$ bulk magnetic systems. Such a torque is considered to be due to the iSGE, which refers to the electrical generation of spin density when a current flows in a system lacking (bulk or interfacial) inversion symmetry. Its reciprocal effect, the spin galvanic effect, is the generation of a charge current in the presence of nonequilibrium spin density (generated, e.g. by photoexcitation). Spin galvanic effect was first predicted by Ivchenko et al. (Ivchenko et al., 1989) and observed by Ganichev et al. (Ganichev et al., 2001, 2002). The iSGE has been predicted originally by Ivchenko and Pikus (Ivchenko and Pikus, 1978), followed by Aronov and Lyanda-Geller
(Aronov and Lyanda-Geller, 1989) and Edelstein (Edelstein, 1990), and observed in non-centrosymmetric systems such as tellurium (Vorobev et al., 1979), strained semiconductors (Kato et al., 2004a) and quantum wells (Ganichev et al., 2004a; Silov et al., 2004; Wunderlich et al., 2004, 2005). More recently, current-driven spin polarization has also been observed at the surface of transition metals (Zhang et al., 2014). In magnets lacking inversion symmetry, such as zinc-blende semiconductors (Bernevig and Vafek, 2005; Garate and MacDonald, 2009; Hals et al., 2010), or magnetic 2D electron gas with Rashba spin-orbit coupling (Manchon and Zhang, 2008; Obata and Tatara, 2008; Tan et al., 2007), the currentdriven spin density can be used to control the magnetic order parameter.

\section{Mechanisms, components, and symmetries}

The iSGE-induced SOT can be derived from the coupled dynamics of the carrier spin density $\mathbf{S}$, brought out of equilibrium by the applied electric field (current), and of the magnetization $\mathbf{m}$. For the sake of the discussion, let us consider the following model Hamiltonian

$$
\hat{H}=\hat{H}_{0}+\hat{H}_{\text {ex }}+\hat{H}_{\text {so }},
$$

where $\hat{H}_{0}$ is the spin-independent part, $\hat{H}_{\mathrm{ex}}=(\Delta / 2) \hat{\boldsymbol{\sigma}} \cdot \mathbf{m}$ is the $s$ - $d$ exchange, and $\hat{H}_{\text {so }}$ is the spin-orbit coupling. From the Ehrenfest theorem, one obtains the coupled dynamic equations

$$
\begin{aligned}
\frac{d \mathbf{S}}{d t} & =\frac{\Delta}{\hbar} \mathbf{S} \times \mathbf{m}+\frac{1}{i \hbar}\left\langle\left[\hat{\boldsymbol{\sigma}}, \hat{H}_{\mathrm{so}}\right]\right\rangle \\
\frac{d \mathbf{m}}{d t} & =\frac{\Delta}{\hbar} \mathbf{m} \times \mathbf{S} .
\end{aligned}
$$

Here $\langle\cdots\rangle$ represents quantum-mechanical averaging over the non-equilibrium carrier states and $\langle\hat{\boldsymbol{\sigma}}\rangle=\mathbf{S}$. The SOT is obtained by introducing the steady-state solution of Eq. (26) $(d \mathbf{S} / d t=0)$ into Eq. (27):

$$
\frac{d \mathbf{m}}{d t}=\frac{\Delta}{\hbar} \mathbf{m} \times \mathbf{S}=\frac{1}{i \hbar}\left\langle\left[\hat{\boldsymbol{\sigma}}, \hat{H}_{\mathrm{so}}\right]\right\rangle .
$$

The left side of Eq. (28) shows explicitly the spin-orbit coupling origin of the SOT. For discerning qualitatively distinct SOT contributions we will now use the middle expression, while the right-hand side can be computed within the linear response theory, accounting for both extrinsic (intraband) and intrinsic (interband) contributions as discussed in Subsection III.A.

Let us first discuss the extrinsic contribution (intraband) which, in the limit of spin-independent disorder, corresponds to the usual Boltzmann contribution. In the limit $\hat{H}_{\mathrm{ex}} \ll \hat{H}_{\mathrm{so}}$, this term is independent of the $s$ - $d$ exchange (Edelstein, 1990; Manchon and Zhang, 2008). A schematic representation of the iSGE spin-polarization $\mathbf{S}$ is shown in Fig. 2(a), bottom panel. For illustration, we 
consider Rashba spin-orbit coupling, $\hat{H}_{\mathrm{so}}=\alpha_{\mathrm{R}} \hat{\boldsymbol{\sigma}} \cdot(\mathbf{p} \times \mathbf{z})$, such that the spins align perpendicular to the wavevector, $\sigma_{\mathbf{k}} \sim \mathbf{z} \times \mathbf{k}$. In the absence of the electric field, $\langle\mathbf{k}\rangle=0$, and the equilibrium distribution of these eigenstate spin vectors adds up into a zero net spin density. Under the applied electric field, however, the states are repopulated with a deficit/excess of left/right moving carriers with respect to the applied electric field. The steady state non-equilibrium distribution is reached when balancing the carrier acceleration in the electric field with scattering against disorder, see Eq. (7). Due to the non-centrosymmetric spin texture of the eigenstates, the non-equilibrium distribution leads to a non-zero net spin density aligned perpendicular to the electric field, $\mathbf{S} \sim \tau \alpha_{\mathrm{R}} \mathbf{z} \times \mathbf{E}$. In analogy to the Boltzmann theory of conductivity, the spin density is proportional to the momentum lifetime $\tau$ and, hence, associated with an extrinsic iSGE. Since we neglected $\hat{H}_{\text {ex }}$ in the carrier Hamiltonian, the iSGE generated spin density $\mathbf{S}$ in this approximation is independent of $\mathbf{m}$ and, when introduced into the middle expression of Eq. (28), yields a FL SOT. Incorporating the exchange field only creates small angular dependance of an otherwise constant spin-accumulation.

Let us now consider the intrinsic (interband) contribution, assuming the same Rashba spin-orbit coupling. Such a term is labeled intrinsic because it has a weak dependence on scattering in metallic systems. This contribution can be also derived from an intuitive picture of the Bloch dynamics of carrier spins. To do so we consider for simplicity the limit $\hat{H}_{e x} \gg \hat{H}_{s o}$ (i.e., the opposite limit than considered above). In equilibrium, the carrier spins are then approximately aligned with the exchange field, $\sigma_{\mathbf{k}} \approx s \mathbf{m}$, independent of their momentum. This is depicted in Figs. 2(b,c), bottom panels, for $\mathbf{m} \| \mathbf{E}$ and $\mathbf{m} \perp \mathbf{E}$, respectively. The Bloch equations describe the carrier spin dynamics during their acceleration in the applied electric field, i.e., between the scattering events. Without loss of generality, we take $\mathbf{E}=E_{x} \mathbf{x}$. For $\mathbf{m} \| \mathbf{E}$, the equilibrium effective magnetic field acting on the carrier spins due to the exchange term is, $\mathbf{B}_{\text {eff }}^{\mathrm{eq}} \approx(\Delta, 0,0)$, in units of energy. During the acceleration in the applied electric field, $\frac{d p_{x}}{d t}=e E_{x}$, and the effective magnetic field acquires a time-dependent $y$-component due to $\hat{H}_{\text {so }}$ for which $\frac{d B_{\text {eff }, y}}{d t}=\left(\alpha_{\mathrm{R}} / \hbar\right) \frac{d p_{x}}{d t}$, as illustrated in Fig. 2(b). For small tilts of the spins from equilibrium, the Bloch equation $\frac{d \sigma_{\mathbf{k}}}{d t}=\frac{1}{\hbar}\left(\boldsymbol{\sigma}_{\mathbf{k}} \times \mathbf{B}_{\text {eff }}\right)$ yields $\sigma_{\mathbf{k}, x} \approx s$, $\sigma_{\mathbf{k}, y} \approx s B_{\mathrm{eff}, \mathrm{y}} / B_{\mathrm{eff}}^{\mathrm{eq}}$, and

$$
\sigma_{\mathbf{k}, z} \approx-\frac{\hbar s}{\left(B_{\mathrm{eff}}^{\mathrm{eq}}\right)^{2}} \frac{d B_{\mathrm{eff}, \mathrm{y}}}{d t}=-\frac{s}{\Delta^{2}} \alpha_{\mathrm{R}} e E_{x} .
$$

The non-equilibrium spin orientation of the carriers acquires a time and momentum independent $\sigma_{\mathbf{k}, z}=\sigma_{z}$ component. For a general angle $\theta_{\mathbf{m}-\mathbf{E}}$ between $\mathbf{m}$ and to $\mathbf{E}$ we obtain,

$$
\sigma_{\mathbf{k}, z}(\mathbf{m})=\sigma_{z}(\mathbf{m}) \approx \frac{s}{\Delta^{2}} \alpha_{\mathrm{R}} e E_{x} \cos \theta_{\mathbf{m}-\mathbf{E}} .
$$

The total non-equilibrium spin polarization, $S_{z}=$ $2 g_{2 D} \Delta \sigma_{z}(\mathbf{m})$, is obtained by integrating $\sigma_{\mathbf{k}, z}$ over all occupied states ( $g_{2 D}$ is the density of states). The nonequilibrium spin polarization produces an out-of-plane field which exerts a torque on the in-plane magnetization. From Eqs. (28) and (30) we obtain an intrinsic DL SOT (Kurebayashi et al., 2014),

$$
\frac{d \mathbf{m}}{d t}=\frac{\Delta}{\hbar}\left(\mathbf{m} \times S_{z} \mathbf{z}\right) \sim \mathbf{m} \times[\mathbf{m} \times(\mathbf{z} \times \mathbf{E})] .
$$

It is worth pointing out the analogy and differences between the intrinsic iSGE and the intrinsic SHE ( $\mathrm{Mu}-$ rakami et al., 2003; Sinova et al., 2004). In the SHE case where $\hat{H}_{\mathrm{ex}}=0$ in the paramagnet, $\mathbf{B}_{\mathrm{eff}}^{\mathrm{eq}}$ depends on the angle $\theta_{\mathbf{k}}$ of the carrier momentum with respect to $\mathbf{E}$ which implies a momentum-dependent $z$-component of the non-equilibrium spin,

$$
\sigma_{\mathbf{k}, z} \approx \frac{s}{\alpha_{\mathrm{R}} k^{2}} \alpha_{\mathrm{R}} e E_{x} \sin \theta_{\mathbf{k}} .
$$

The same spin rotation mechanism that generates the uniform bulk spin accumulation in the case of the intrinsic iSGE in a FM [Fig. 2(b)] is responsible for the scattering-independent spin current of the SHE in a paramagnet (Sinova et al., 2004). Note that the SHE spin current yields zero spin accumulation in the bulk and a net SHE spin-polarization occurs only at the edges of the conduction channel.

\section{Inverse spin galvanic torque in a magnetic $2 \mathrm{D}$ electron gas}

Because of the symmetry present in most bilayer systems considered in experiments, the Rashba spin-orbit coupling is the natural model to study, and therefore the iSGE-SOT has been alternatively called Rashba-SOT in this context. As discussed above, the Rashba torque can possess two components corresponding to the FL and DL torques, see Eq. (1). While the origin of the FL torque is well understood and consistently attributed to the extrinsic intraband iSGE (Rashba-Edelstein) effect (Edelstein, 1990), several mechanisms contribute to the DL torque. The different contributions have been investigated using semiclassical Boltzmann transport equation (van der Bijl and Duine, 2012; Kim et al., 2013b, 2012b; Lee et al., 2015; Manchon and Zhang, 2008; Matos-Abiague and Rodriguez-Suárez, 2009; Tan et al., 2007), or quantummechanical Kubo formula approaches (Li et al., 2015; Pesin and MacDonald, 2012a; Qaiumzadeh et al., 2015; Wang et al., 2012b, 2014).

As discussed above, interband transitions produce an intrinsic DL torque in the limit of weak scattering (Freimuth et al., 2014b; Kurebayashi et al., 2014), and can be related to the Berry curvature of the electronic band structure in the mixed spin-momentum phase space (Freimuth et al., 2014b; Kurebayashi et al., 2014; Lee 
et al., 2015; Li et al., 2015). As a result, one can expect "hot spots" in the band structure, i.e., points where neighboring bands get very close to each other, to give very large contribution, similarly to the case of intrinsic SHE (Tanaka et al., 2008). Notice that in the specific case of the pure 2D Rashba gas, vertex corrections cancel the intrinsic DL torque unless the momentum relaxation time is spin-dependent (Qaiumzadeh et al., 2015), similar to the cancelation occurring for the intrinsic SHE in pure 2D Rashba gas (Inoue et al., 2004). Nevertheless, such cancellations are highly sensitive to this specific model band structure and can be considered as accidental, as discussed by Sinova et al., 2015 in the context of intrinsic SHE.

Extrinsic iSGE mechanisms related to spin scattering were also theoretically shown to generate a DL component of the SOT. In (Kim et al., 2012b), the DL term arises from the momentum-scattering induced spin relaxation, an effect initially proposed in metallic spin-valves and DWs (Zhang et al., 2002; Zhang and Li, 2004). In fact, when a non-equilibrium spin density $\mathbf{S}$ is injected into a magnet, spin relaxation generates a corrective term of the form $\sim \beta_{\text {sf }} \mathbf{m} \times \mathbf{S}$, where $\beta_{\text {sf }}$ is a parameter that depends on the ratio between spin precession and spin-flip scattering. In other works, this component is obtained within a quantum kinetic formalism and ascribed to spindependent carrier lifetimes or to a term arising from the weak-diffusion limit which in the leading order is proportional to a constant carrier lifetime (Pesin and MacDonald, 2012a; Wang and Manchon, 2012; Wang et al., 2014).

Finally, we stress out that the coefficients $\tau_{\mathrm{FL}, \mathrm{DL}}$ are in principle angular dependent and display terms proportional to $\sin ^{2 n} \theta_{\mathbf{m}-\mathbf{E}}, n \in \mathbb{N}^{*}$. This angular dependence reflects the distortion of the band structure when changing the magnetization direction (Lee et al., 2015), as well as the anisotropic spin relaxation in the system due to D'yakonov-Perel's mechanism (Ortiz Pauyac et al., 2013).

\section{Non-centrosymmetric bulk magnets}

Dilute magnetic semiconductor (Ga,Mn)As has been a test-bed material for observing and exploring the bulk SOT. Hence, unlike the case of bilayer systems, all torques observed in these bulk materials arise internally with no contribution from externally injected spin currents. Current-driven torques in dilute magnetic semiconductors were first studied by Bernevig and Vafek (Bernevig and Vafek, 2005). The authors considered the Kohn-Luttinger Hamiltonian in the spherical approximation, augmented by a k-linear spin-orbit coupling term arising from strain of the form $\boldsymbol{\lambda} \cdot \hat{\mathbf{J}}$, where $\lambda_{x}=C_{4}\left(\epsilon_{x y} k_{y}-\epsilon_{x z} k_{z}\right)$ and $\lambda_{y, z}$ are obtained from cyclic permutation of indices. The current-driven spin density reads

$$
\mathbf{S}=-\frac{e \tau}{\hbar^{2}} \frac{15}{4}\left(\sum_{s= \pm 1} \frac{\sqrt{3 n / \pi}}{\left(\gamma_{1}+2 s \gamma_{2}\right)^{3 / 2}}\right)^{2 / 3}\left(e \mathbf{E} \cdot \nabla_{\mathbf{k}}\right) \boldsymbol{\lambda}
$$

where $n$ is the charge density. Because in their calculation they did not consider an exchange coupling directly, the torque induced by this iSGE is therefore a FL torque. The intrinsic DL torque originating from interband transitions was later proposed by Kurebayashi et al. (2014) to interpret the experimental observation of such a DL torque in (Ga,Mn)As, see Fig. 11. The theoretical investigation of SOT in dilute magnetic semiconductors was also pursued by Li et al. (2015, 2013). Besides some subtleties related to the complex band structure, the numerical results obtained by these authors qualitatively confirm the general picture obtained in the context of the magnetic Dresselhaus and Rashba gas.
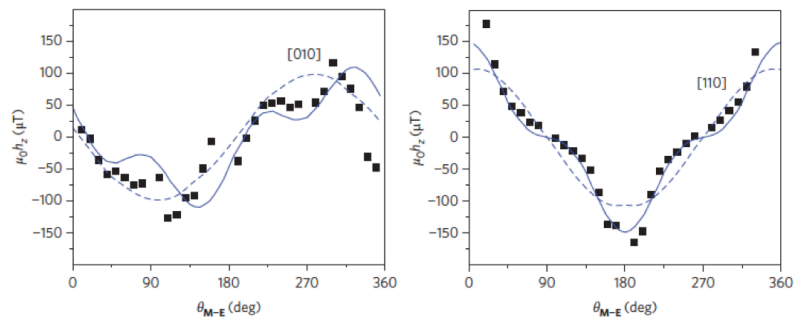

FIG. 11 (Color online) Microscopic modeling of the intrinsic SOT in bulk (Ga,Mn)As. The solid lines correspond to the numerical results and the symbols correspond to the experimental data. The dashed lines correspond to the free electron approximation $\left(\gamma_{1}=\gamma_{2}=\gamma_{3}\right)$. From (Kurebayashi et al., 2014).

Apart from (Ga,Mn)As, the SOT has been studied in several other bulk systems. One of them is the FM NiMnSb (Ciccarelli et al., 2016). This half-heusler material has the same crystalline symmetry as zinc-blende (Ga,Mn)As; however, it is not a dilute-moment random alloy like (Ga,Mn)As, but a dense-moment ordered compound. Despite these differences, the SOTs found in $\mathrm{NiMnSb}$ are quite similar to those in (Ga,Mn)As. This shows, in agreement with other calculations, that the torque is to large extent determined by symmetry.

\section{E. Microscopic modeling of spin-orbit torques in bilayer systems}

Experimentally, although following after the studies in bulk magnets, SOTs have been most extensively studied in NM/FM bilayer (or multilayer) structures (Liu et al., 2012b; Miron et al., 2011a, 2010). Theories of SOT in bilayer systems based on iSGE and SHE as exposed in the previous sections present two major limitations. First, 
both mechanisms formally apply in very distinct situations: SHE-induced spin-current is a bulk effect, and up till now only very few publications have addressed the nature of the SHE SOT in ultrathin magnetic multilayers (Amin and Stiles, 2016a,b; Haney et al., 2013b; Kim et al., 2017b; Saidaoui and Manchon, 2016; Wang et al., 2016a). In contrast, iSGE in magnetic multilayers is often modeled using Rashba interaction, which applies to $2 \mathrm{D}$ gases and ideally sharp interfaces only. A second flaw is the overlook of the details of the interfacial orbital overlap, which can be quite subtle in transition metal interfaces (Blügel and Bihlmayer, 2007; Grytsyuk et al., 2016).

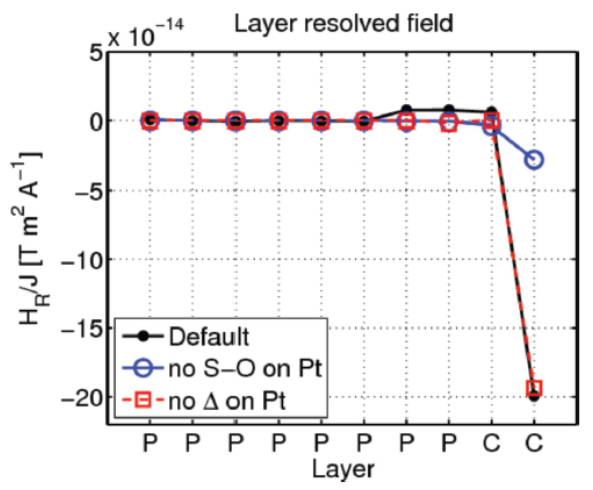

FIG. 12 (Color online) Layer-resolved FL torque in normal $\mathrm{Pt} / \mathrm{Co}(111)$ (black symbols), when turning off the induced magnetization on Pt (red symbols) and when turning off the spin-orbit coupling on Pt (blue symbols). From (Haney et al., 2013a)

To overcome these issues, SOTs in $\mathrm{Co} / \mathrm{Pt}$ bilayer systems have been computed within the relaxation time approximation using an ab initio DFT description of the whole bilayer structure (Freimuth et al., 2014b, 2015; Haney et al., 2013a). In Haney et al. (2013a), SHE or intrinsic contributions to iSGE are disregarded. In spite of the high complexity of the band structure, these calculations confirm the intuitive picture elaborated based on the Rashba model (Haney et al., 2013a). In particular, they show that SOT is mostly driven by spin-orbit coupling in $\mathrm{Pt}$, while the influence of induced magnetization is negligible, see Fig. 12. Moreover, the torque acquires a non-trivial angular dependence, and depends dramatically on the quality of the interface.

Alternatively, Freimuth et al. (Freimuth et al., 2014b, 2015; Géranton et al., 2015) computed the full KuboBastin formula, thereby accounting for both SHE or intrinsic iSGE. These calculations confirmed that SOTs are composed of both FL and DL torques, the latter being produced by interband transitions only, see Fig. 13. An interesting aspect revealed through these calculations is the high sensitivity of SOTs to interfacial engineering. In fact, the authors found that by capping the Co layer by either $\mathrm{Al}$ or $\mathrm{O}$ atoms, the $\mathrm{DL}$ torque is only slightly affected (its magnitude changes up to $50 \%$ - see Fig. 13) while the FL torque is dramatically altered and can even change its sign. In a recent work, these authors also investigated the impact of impurities and phonons on the SOT within the Korringa-Kohn-Rostoker Green's functions method (Géranton et al., 2016).

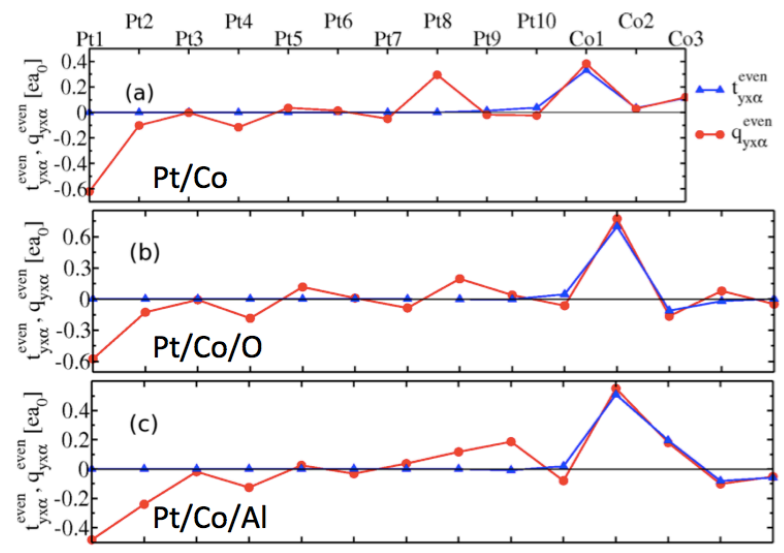

FIG. 13 (Color online) Layer-resolved DL torque $t_{x y}^{\text {even }}$ (red symbols) and spin current $q_{x y}^{\text {even }}$ (blue symbols) in (a) Pt/Co, (b) $\mathrm{Pt} / \mathrm{Co} / \mathrm{O}$ and (c) $\mathrm{Pt} / \mathrm{Co} / \mathrm{Al}$. From (Freimuth et al., 2014b)

Finally, in one of the few calculations that goes beyond the constant relaxation time approximation, Wimmer et al., 2016 calculated the torque in $\mathrm{Pt} / \mathrm{Fe}_{x} \mathrm{Co}_{1-x} / \mathrm{Cu}$ superlattice using the Kubo-Bastin formula and coherent potential approximation to account for the alloy disorder. This allows for treating extrinsic scattering mechanisms (i.e., side jump and skew scattering) within the framework of DFT.

\section{F. Antiferromagnets}

Since the first proposal of STT in AFs (MacDonald and Tsoi, 2011; Núñez et al., 2006), several configurations have been theoretically investigated to enable large spin torque efficiency. In the course of the search for such torques it was realized that in order to efficiently manipulate the order parameter of a collinear, bipartite AF one needs a torque that corresponds to a staggered effective magnetic field, i.e., a field with an opposite sign on the two magnetic sublattices. Such a field, unlike a homogeneous field, couples directly to the Néel order. The torque resulting from a staggered field has been referred to as Néel SOT (Železný et al., 2017, 2014).

The DL torque due to a spin current (from a SHE or a FM polarizer) is a Néel torque, assuming that it has the same form on each magnetic sublattice as in FMs, i.e., $\mathbf{T}_{a}=\tau_{\mathrm{DL}} \mathbf{m} \times(\mathbf{m} \times \boldsymbol{\zeta})$. This form of the torque has been predicted theoretically (Cheng et al., 
2014; Gomonay et al., 2012; Železný et al., 2014) and it was shown that it can indeed efficiently manipulate the AF order (Gomonay et al., 2012; Gomonay and Loktev, 2010). A recent drift-diffusion theory confirmed that spin current injected from an adjacent FM polarizer or induced by SHE indeed generates such a Néel DL torque (Manchon, 2017). An experimental indication of the presence of the SHE generated SOT in an NM/AF bilayer was reported in Reichlova et al. (2015).

The bulk SOT can also have the Néel order form if the current-induced spin-polarization has an opposite sign on the two sublattices (Železný et al., 2014). In a collinear $\mathrm{AF}$, the two sublattices with opposite magnetizations are connected by some symmetry operation. Typically, this is either a translation or inversion. This symmetry operation combined with time-reversal is then a symmetry of the magnetic system which, using Eqs. (17) and (18), relates the current-induced spin-polarizations on the two sublattices (Železný et al., 2017). If the sublattices are connected by translation then

$$
\begin{aligned}
\chi_{B, a}^{\text {even }} & =\chi_{B, b}^{\text {even }}, \\
\chi_{B, a}^{\text {odd }} & =-\chi_{B, b}^{\text {odd }},
\end{aligned}
$$

where $a, b$ denotes the two sublattices. If they are connected by inversion

$$
\begin{gathered}
\chi_{B, a}^{\text {even }}=-\chi_{B, b}^{\text {even }}, \\
\chi_{B, a}^{\text {odd }}=\chi_{B, b}^{\text {odd }} .
\end{gathered}
$$

Thus in both cases there exists both a staggered component and a uniform component of the current induced spin-polarization and the corresponding effective field. For the magnetic dynamics only the staggered component will have an appreciable effect for the achievable magnitudes of the effective fields. Since different component is staggered in the two cases, the dynamics will differ. As discussed in Section III.B, the even field typically has a FL character, whereas the odd field is commonly DL. Thus in systems where magnetic sublattices are connected by translation we can expect efficient DL torque, whereas in systems where the sublattices are connected by inversion a FL torque is expected.

SOTs in AFs have been first studied in two tightbinding models (Železný et al., 2017, 2014): (i) the AF 2D Rashba gas and (ii) the bulk $\mathrm{Mn}_{2} \mathrm{Au}$. Both systems possess collinear antiferromagnetism. They together illustrate the two main types of symmetry discussed above. In the Rashba model the two sublattices are connected by translation and thus the lowest order Néel order SOT has a DL character. In the $\mathrm{Mn}_{2} \mathrm{Au}$ crystal, on the other hand, the two sublattices are connected by inversion and the lowest order Néel torque has consequently a FL character. Microscopic calculations based on the Kubo formula with constant relaxation time indeed show that the Néel SOT in the Rashba model is primarily DL, whereas in $\mathrm{Mn}_{2} \mathrm{Au}$ it is predominantly of FL character.
The origin of the FL torque in the $\mathrm{Mn}_{2} \mathrm{Au}$ crystal can be understood in terms of the symmetry of the nonmagnetic crystal. Without magnetism, the crystal has inversion symmetry and thus there is no net current-induced spin-polarization. Yet, the Mn sublattices each have locally broken inversion symmetry and thus can have current-induced spin-polarizations that have to be precisely opposite. An intuitive explanation of this behavior is that the local inversion breaking is opposite for the two sublattices and thus the induced spin-polarizations are also opposite. When magnetism is added these opposite spin-polarizations generate a staggered effective field. In the Rashba model on the other hand, the inversion breaking is the same for both sublattices and thus the field generating the FL torque is not staggered. On the other hand, the field generating the DL torque is staggered, since it is proportional to the magnetic moment which is staggered in the AF. The FL torque in $\mathrm{Mn}_{2} \mathrm{Au}$ has a Rashba-like symmetry, i.e., the effective field is on each sublattice proportional to $\boldsymbol{\zeta}$. This is because the local symmetry of the Mn sublattices is the same as that of the 2D Rashba model.

Following the calculations based on tight-binding models, the SOT was also calculated in AFs using DFT. Such calculations were done for $\mathrm{Mn}_{2} \mathrm{Au}$ (Železný et al., 2017) and CuMnAs (Wadley et al., 2016), which has a symmetry analogous to $\mathrm{Mn}_{2} \mathrm{Au}$. These results agree well with the tight-binding calculations in term of the magnetization and current dependence and in addition show a relatively large torque. The magnitude of the effective field is around $2 \mathrm{mT}$ per $10^{7} \mathrm{Acm}^{-2}$ current density for $\mathrm{Mn}_{2} \mathrm{Au}$ and $3 \mathrm{mT}$ per $10^{7} \mathrm{Acm}^{-2}$ for CuMnAs. The switching due to this FL Néel order torque has been observed in CuMnAs (Wadley et al., 2016) and subsequently in $\mathrm{Mn}_{2} \mathrm{Au}$ (Bodnar et al., 2017; Meinert et al., 2017).

\section{G. Three-dimensional topological insulators}

Topological insulators are a class of materials displaying intriguing properties such as insulating bulk and conductive chiral and helical surfaces (Hasan and Moore, 2011; Qi and Zhang, 2011; Wehling et al., 2014). Considering the large spin-charge conversion efficiency recently reported in these systems (see Section IV.C.4), they deserve special attention. The category of topological materials we are interested in are characterized by timereversal symmetry and helical surface states: their low energy surface states are represented by a Dirac Hamiltonian of the form $\sim p_{i} \sigma_{j}$ [see Fig. 14(a, b)]. When electrons flow on the surface of these systems, they acquire a non-equilibrium spin density, similar to the case of the 2D Rashba gas, as demonstrated in a $\mathrm{Bi}_{2} \mathrm{Se}_{3}$ slab using ab initio calculations (Chang et al., 2015). Since the strength of the spin-momentum coupling is quite large $\left(\sim 4 \times 10^{10} \mathrm{eV} \cdot \mathrm{m}\right.$ at $\mathrm{Bi}_{2} \mathrm{Se}_{3}$ surfaces, comparable to $\mathrm{Bi} / \mathrm{Ag}$ 
surfaces, and two orders of magnitude larger than in InAlAs/InGaAs 2D gases), iSGE is expected to be very large. In addition, the absence of bulk conduction in ideal topological insulators further strongly enhances the spin-charge conversion efficiency.

Spin-charge conversion processes in topologicalinsulator/FM bilayers have been studied by several authors (Fujimoto and Kohno, 2014; Linder, 2014; Sakai and Kohno, 2014; Taguchi et al., 2015; Tserkovnyak et al., 2015). The low energy Hamiltonian reads $\hat{H}=$ $v \hat{\boldsymbol{\sigma}} \cdot(\hat{\mathbf{p}} \times \mathbf{z})+\frac{\Delta}{2} \hat{\boldsymbol{\sigma}} \cdot \mathbf{m}$, where the first term models the Dirac cone and the second term is the exchange. The eigenenergies read

$$
\epsilon_{k}^{s}=s \sqrt{\left(v k_{x}+\frac{\Delta}{2} m_{y}\right)^{2}+\left(v k_{y}-\frac{\Delta}{2} m_{x}\right)^{2}+\frac{\Delta^{2}}{4} m_{z}^{2}(38)}
$$

When $m_{z} \neq 0$ the surface states are gapped, whereas when $m_{z}=0$, the origin of the band dispersion is only shifted in the $\mathbf{k}$-plane. If the Fermi energy lies in the gap, quantum anomalous Hall effect emerges, accompanied by a quantized magnetoelectric effect, $\mathbf{S}=-\frac{e \hbar}{2 \pi v} \mathbf{E}$ (Nomura and Nagaosa, 2011; Qi et al., 2008). On the other hand, when the Fermi level lies above the gap, the system is metallic and the SOT possesses both FL and DL components of the form (Garate and Franz, 2010; Ndiaye et al., 2017)

$$
\mathbf{T}=\tau_{\mathrm{FL}} \mathbf{m} \times \boldsymbol{\zeta}+\tau_{\mathrm{DL}} m_{z} \mathbf{m} \times(\mathbf{z} \times \boldsymbol{\zeta}),
$$

where $\boldsymbol{\zeta} \| \mathbf{z} \times \mathbf{E}$. While the FL torque arises from the conventional extrinsic iSGE, the DL torque arising from the intrinsic interband contribution is proportional to $m_{z}$ and therefore vanishes when the magnetization lies in the plane of the surface, in sharp contrast with the usual DL torque given in Eq. (1) (Ndiaye et al., 2017).

The calculations discussed above are based on the 2D Dirac gas model, i.e, assuming that the transport is confined to the interface and that surface states remain intact in the presence of the proximate FM layer. Such a model presents two major drawbacks though. First, orbital hybridization between the transition metal and the topological insulator substantially alters the surface states at Fermi energy. The presence of magnetic adatoms shifts the Dirac cone downward in energy (Honolka et al., 2012; Scholz et al., 2012; Ye et al., 2012), and favor the presence of additional metallic bands with Rashba-like character across the Fermi level (MarmolejoTejada et al., 2017; Zhang et al., 2016a).

A second limitation comes for the three dimensional nature of the transport. Indeed, most experiments involve topological insulators with sizable bulk conductivity, suggesting that bulk states might participate to the spin-charge conversion mechanism. Spin transport in such systems has been recently investigated using driftdiffusion model (Fischer et al., 2016), non-equilibrium Green's function technique (Mahfouzi et al., 2016) and Kubo formula (Ghosh and Manchon, 2017b). The first two studies show that spin diffusion in the FM and spinflip scattering at the interface can enhance the DL torque. The latter work accounts for interfacial and bulk transport on equal footing and demonstrates that a large DL torque is driven by the Berry-curvature of the interfacial states, whereas the SHE of the bulk states is inefficient.

Finally, spin-orbit charge pumping, the reciprocal effect of SOT, has also been investigated theoretically in topological insulators (Mahfouzi et al., 2014; Ueda et al., 2012), providing a charge current of the form $\mathbf{j}_{\mathrm{c}}=(\hbar / 2)\left(\tau_{\mathrm{DL}} m_{z} \partial_{t} \mathbf{m}+\tau_{\mathrm{FL}} \mathbf{z} \times \partial_{t} \mathbf{m}\right)($ Ndiaye et al., 2017$)$. A direct consequence of this current is the induction of an anisotropic magnetic damping on the FM layer (Yokoyama et al., 2010).
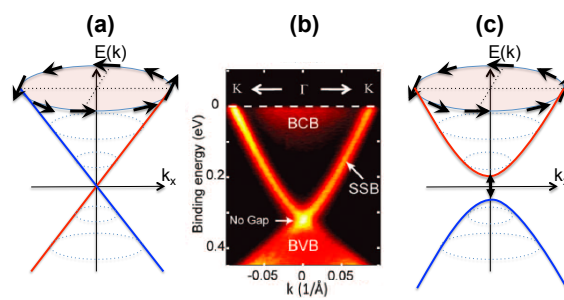

(d)

FIG. 14 (Color online) (a) Schematics of the band dispersion at the $2 \mathrm{D}$ surface of a topological insulator; (c) the same with a perpendicular magnetic field. (b) Band dispersion measured by Angle Resolved Photoemission Spectroscopy of the surface state of $\mathrm{Bi}_{2} \mathrm{Se}_{3}$ (Chen et al., 2010); (d) the same for Fe-doped $\mathrm{Bi}_{2} \mathrm{Se}_{3}$.

\section{H. Other spin-orbit torques}

\section{Anisotropic magnetic tunnel junctions}

SOT has also been recently proposed in magnetic tunnel junctions (MTJs) composed of a single FM with interfacial spin-orbit coupling (Manchon, 2011a). Such systems display tunneling anisotropic magnetoresistance, i.e., a change of resistance when varying the magnetization direction (Gould et al., 2004; Park et al., 2008), see Fig. 15(a). One naturally expects that spin-polarized electrons impinging on the spin-orbit coupled interface precess about the spin-orbit field, resulting in a torque on the local magnetization. This SOT is of the form given by Eq. (1) with $\boldsymbol{\zeta}=\mathbf{z}$. The FL torque possesses an equilibrium contribution [which is nothing but the perpendicular magnetic anisotropy (Manchon, 2011b)] and the DL torque is purely non-equilibrium. Both non-equilibrium torques are linear as a function of the bias voltage, but the magnitude of the non-equilibrium torques are quadratic in Rashba parameter, see Fig. 15. A similar idea has been proposed by Mahfouzi et al. (2012) by considering a topological insulator as a tunnel barrier. 

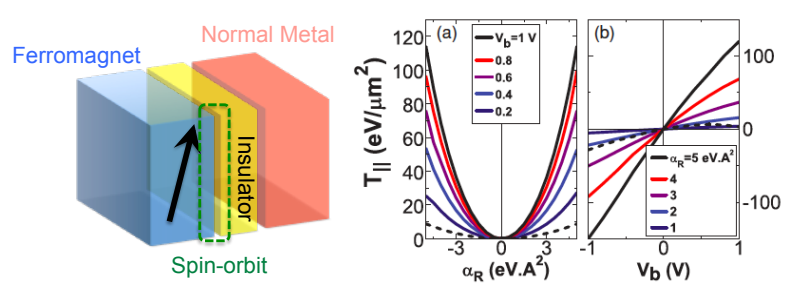

FIG. 15 (Color online) (Left pannel) Schematics of a MTJ composed of a FM and a NM separated by a tunnel barrier. Interfacial spin-orbit coupling is present at the interface between the FM and the tunnel barrier. (Right pannel) Rashba dependence (a) and bias dependence (b) of the inplane torque. From (Manchon, 2011a)

\section{Two dimensional topological insulators and hexagonal lattices}

SOT and spin-orbit charge pumping have also been studied in various configurations involving 2D topological insulators (Soleimani et al., 2017). These studies reveal that SOT experiences a significant enhancement depending on the topological phase ( $\mathrm{Li}$ and Manchon, 2016; Mahfouzi et al., 2010): the emergence of edge currents promotes a quantized charge pumping when the magnetization is perpendicular to the plane. Such investigations have been recently extended to AF 2D topological insulators, where a time-reversal combined with a half unit cell translation is a symmetry of the system which preserves topological protection, despite the broken time-reversal symmetry of the magnetic state (Ghosh and Manchon, 2017a).

SOTs have also been theoretically studied in magnetic 2D hexagonal lattices such as, but not limited to, graphene, silicene, germanene, stanene, transition metal dichalcogenides etc. (Dyrdał and Barnas, 2015; Li et al., 2016a). The parametric dependencies of the torque in these materials do not significantly differ from the Rashba model. Nonetheless, in these systems the low-energy transport occurs mostly through two independent valleys, which opens the possibility to obtain valley-dependent SOTs.

\section{Spin-transfer torque assisted by spin-orbit coupling}

When non-equilibrium spin density penetrates a magnetic with spin-orbit coupling, it precesses around the local spin-orbit field. This precession results in additional angular dependences of the SOT in Rashba (Lee et al., 2015) and Kohn-Luttinger systems as discussed above (Kurebayashi et al., 2014; Li et al., 2013). Interestingly, Haney and Stiles (2010) showed that in a metallic spinvalve where spin-orbit coupling is present, such a precession results in an overall STT enhancement. Considering the general Hamiltonian, Eq. (25), with $\hat{H}_{\text {so }}=\xi \hat{\mathbf{L}} \cdot \hat{\boldsymbol{\sigma}}$, the total angular momentum $\hat{\mathbf{J}}=\hat{\mathbf{L}}+\hat{\boldsymbol{\sigma}}$ obeys to continuity equation

$$
\frac{d \hat{\mathbf{J}}}{d t}-\nabla \cdot \mathcal{J}_{\mathbf{J}}=-\hat{\boldsymbol{\tau}}_{\mathrm{STT}}-\hat{\boldsymbol{\tau}}_{\mathrm{lat}}
$$

where $\mathcal{J}_{\mathbf{J}}$ is the total angular momentum current density, $\hat{\boldsymbol{\tau}}_{\mathrm{STT}}$ is the STT and $\hat{\boldsymbol{\tau}}_{\text {lat }}=i\langle[\hat{H}, \hat{\mathbf{L}}]\rangle / \hbar$ is the mechanical torque. The latter is nothing but the precession of itinerant spins about the spin-orbit field, such that the total spin torque in a spin-valve survives away from the interface, see Fig. 16(a). Due to this additional precession, the total torque extends over the whole thickness of the free layer, as displayed in Fig. 16(b). A similar effect has been identified in magnetic DWs where spin-orbit coupling enhances spin reflection and thereby STT (Nguyen et al., 2007; Yuan and Kelly, 2016). (a)

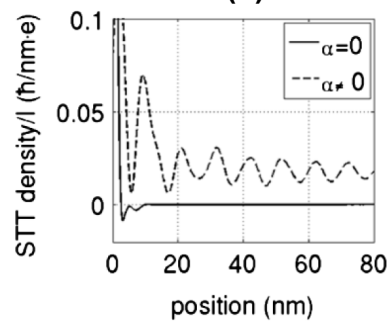

(b)

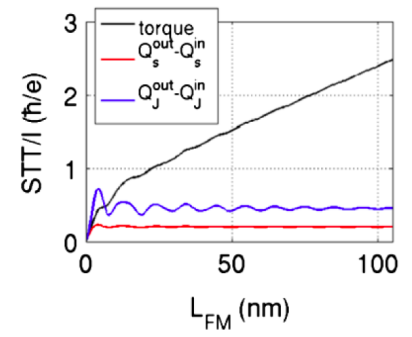

FIG. 16 (Color online) (a) Spatial profile of the transverse spin density injected in the free layer of a (Ga,Mn)As-based MTJ in the presence and absence of spin-orbit coupling in the band structure; (b) Spin torque efficiency as a function of the free layer thickness. From (Haney and Stiles, 2010).

\section{SPIN-ORBIT TORQUES IN MAGNETIC MULTILAYERS}

This section reviews recent experimental progress in the measurement and characterization of SOT in multilayer systems. We first introduce the phenomenological description of SOT commonly used in experiments (Subsection IV.A) and the main techniques employed to measure SOT (Subsection IV.B). Next, we present a survey of different materials, namely NM/FM, AF/FM, semiconductor/FM, and topological-insulator/FM layers (Subsection IV.C), summarizing the most salient features of the SOT observed in these systems. Finally, we describe the SOT-induced magnetization dynamics (Subsection IV.D) and switching (Subsection IV.E), and conclude by highlighting examples and perspectives for the implementation of SOT in magnetic devices (Subsection IV.F).

\section{A. Phenomenological description}

Current-injection in heterostructures composed of a magnetic layer adjacent to a nonmagnetic conductor with 

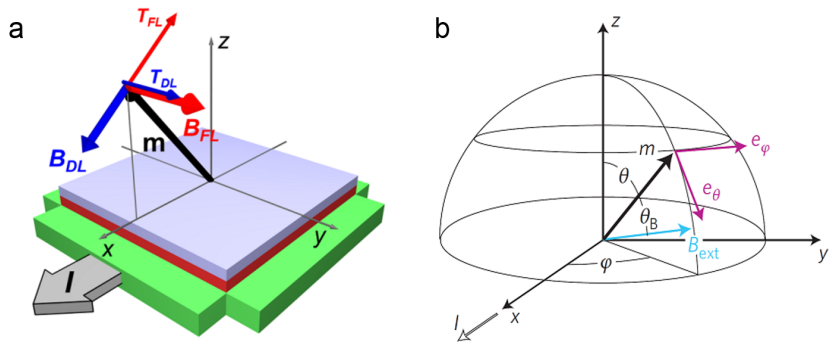

FIG. 17 (Color online) (a) Spin-orbit torques and corresponding effective fields measured in $\mathrm{Pt} / \mathrm{Co} / \mathrm{AlO}_{x}$ layers when the magnetization is tilted parallel to the current direction. (b) Schematic of the coordinate system.

either bulk or interface spin-orbit coupling gives rise to a transverse spin accumulation $\boldsymbol{\zeta} \| \mathbf{z} \times \mathbf{j}_{\mathrm{c}}$ at the interface of the magnetic layer. This spin accumulation induces both DL and FL SOT components, as shown in Fig. 17(a), and described by Eq. (1). For experimental purposes, it is useful to introduce two effective magnetic fields, $\mathbf{B}_{\mathrm{DL}, \mathrm{FL}}$, which correspond to the DL and FL torques and defined by $\mathbf{T}_{\mathrm{DL}, \mathrm{FL}}=\mathbf{M} \times \mathbf{B}_{\mathrm{DL}, \mathrm{FL}}$. The advantage of the effective field formulation in the SOT characterization is that their action on the magnetization can be directly compared to that of a reference external field of known magnitude and direction. To the lowest order in the magnetization, for a current $\mathbf{j}_{\mathbf{c}} \| \mathbf{x}$, Eq. (1) gives

$$
\begin{aligned}
& \mathbf{B}_{\mathrm{FL}}=B_{\mathrm{FL}} \mathbf{y}, \\
& \mathbf{B}_{\mathrm{DL}}=B_{\mathrm{DL}} \mathbf{m} \times \mathbf{y},
\end{aligned}
$$

where the field amplitudes are simply $B_{\mathrm{FL}}=\tau_{\mathrm{FL}}$ and $B_{\mathrm{DL}}=\tau_{\mathrm{DL}}$ if the torques are calculated for the unitary magnetization $\mathbf{m}$, as assumed in Eq. (1). ${ }^{1}$ Thus, for positive values of the SOT coefficients $\tau_{\mathrm{FL}}$ and $\tau_{\mathrm{DL}}, \mathbf{B}_{\mathrm{FL}} \| \mathbf{y}$ whereas $\mathbf{B}_{\mathrm{DL}}$ rotates clockwise in the $x z$ plane, corresponding to $\mathbf{T}_{\mathrm{DL}} \|-\mathbf{y}$. Figure $17(\mathrm{a})$ shows the orientation of the torques and effective fields for the model system $\mathrm{Pt} / \mathrm{Co} / \mathrm{AlO}_{x}$, in which $\tau_{\mathrm{FL}}>0$ and $\tau_{\mathrm{DL}}<0$ (Garello et al., 2013). Typical values of $B_{\mathrm{FL}, \mathrm{DL}}$ in NM/FM systems are in the range $0.1-10 \mathrm{mT}$ for a current density $j_{c}=10^{7} \mathrm{~A} / \mathrm{cm}^{2}$. Note also that the Oersted field due to the current flowing in the nonmagnetic layer produces an additional field $B_{O e} \approx \mu_{0} j_{c} t_{N} / 2$ antiparallel (parallel) to $\mathbf{y}$ for bottom (top) stacking relative to the magnetic layer.

In the typical NM/FM bilayers, the measurable effect of the SOT on the magnetization scale with the current and the volume of the FM. It is thus useful to define the

\footnotetext{
1 To emphasize the direction of the effective fields in perpendicularly magnetized layers, $B_{\mathrm{FL}}$ and $B_{\mathrm{DL}}$ are sometimes called "transverse field" $\left(H_{\mathrm{T}}\right)$ and "longitudinal field" $\left(H_{\mathrm{L}}\right)$, respectively (Kim et al., 2013a).
}

spin torque efficiencies

$$
\xi_{\mathrm{DL}, \mathrm{FL}}^{j}=\frac{2 e}{\hbar} M_{\mathrm{s}} t_{F} \frac{B_{\mathrm{DL}, \mathrm{FL}}}{j_{c}},
$$

where $M_{\mathrm{s}}$ is the saturation magnetization and $t_{F}$ the thickness of the FM. The parameters $\xi_{\mathrm{DL}, \mathrm{FL}}^{j}$ represent the ratio of the effective spin current absorbed by the FM relative to the charge current injected in the NM layer, and can thus be considered as effective spin Hall angles for a particular combination of NM and FM. In the pure SHE-SOT picture, $\xi_{\mathrm{DL}}^{j}$ is equal to the bulk spin Hall angle of the NM in the limit of a transparent interface and negligible spin memory loss. Although the SOT efficiencies are useful parameters to compare the strength of the SOT in different systems, ambiguities remain on how to estimate $j_{c}$ in layered heterostructures. While some authors consider $j_{c}$ to be the average current density, others apply a parallel resistor model to separate the currents flowing in the NM and FM layers. However, thickness inhomogeneities and interface scattering can significantly alter the current distribution in NM/FM systems relative to such a model. Even in homogeneous films, the conductivity is a strong function of the thickness (Fuchs, 1938; Sambles, 1983) so that $j_{c}$ changes in the bulk and interface regions of a conductor. For these reasons, the current normalization should be performed with care. Alternatively, it is possible to measure the torque efficiency per unit electric field (Nguyen et al., 2016)

$$
\xi_{\mathrm{DL}, \mathrm{FL}}^{E}=\frac{2 e}{\hbar} M_{\mathrm{s}} t_{F} \frac{B_{\mathrm{DL}, \mathrm{FL}}}{E}=\xi_{\mathrm{DL}, \mathrm{FL}}^{j} / \rho,
$$

where $E=\rho j_{c}$ is the electric field driving the current, which is independent of the sample thickness and can be easily adjusted in voltage-controlled experiments. Note that, in the framework of the SHE-SOT model, $\xi_{\mathrm{DL}}^{E}$ can be considered as an effective spin Hall conductivity.

Equations (1) and (42) correspond to the lowest order terms of the SOT, which are sufficient to describe many experimental results, at least on a qualitative level. On a more general level, however, higher order terms in the magnetization are allowed by symmetry. The typical polycrystalline NM/FM metal bilayers have $\mathrm{C}_{v}$ symmetry, corresponding to broken inversion symmetry along the $\mathbf{z}$-axis and in-plane rotational symmetry. For such systems, the torques can be decomposed into the following terms (Garello et al., 2013),

$$
\begin{aligned}
\mathbf{T}_{\mathrm{FL}} & =\left[\tau_{\mathrm{FL}}^{\{0\}}+\sum_{n \geq 1} \tau_{\mathrm{FL}}^{\{2 n\}}(\sin \theta)^{2 n}\right] \mathbf{y} \times \mathbf{m} \\
& +\mathbf{m} \times(\mathbf{z} \times \mathbf{m}) m_{x} \sum_{n \geq 1} \tau_{\mathrm{FL}}^{\{2 n\}}(\sin \theta)^{2(n-1)}, \\
\mathbf{T}_{\mathrm{DL}} & =\tau_{\mathrm{DL}}^{\{0\}} \mathbf{m} \times(\mathbf{y} \times \mathbf{m}) \\
& +m_{x} \mathbf{z} \times \mathbf{m} \sum_{n \geq 1} \tau_{\mathrm{DL}}^{\{2 n\}}(\sin \theta)^{2(n-1)}
\end{aligned}
$$


where $\theta$ is the polar angle of the magnetization defined in Fig. 17(b). This formula is general and does not depend on the particular mechanism, SHE or iSGE, responsible for the spin accumulation. In a material displaying additional symmetries, such as epitaxial films or single crystals, additional angular dependencies arise (Hals and Brataas, 2013; Wimmer et al., 2016; Železný et al., 2017). This complex dependence of the SOT on the magnetization direction is best captured by writing the effective fields in spherical coordinates,

$$
\begin{gathered}
\mathbf{B}_{\mathrm{DL}}=B_{\mathrm{DL}}^{\theta} \cos \varphi \mathbf{e}_{\theta}-B_{\mathrm{DL}}^{\varphi} \cos \theta \sin \varphi \mathbf{e}_{\varphi}, \\
\mathbf{B}_{\mathrm{FL}}=-B_{\mathrm{FL}}^{\theta} \cos \theta \sin \varphi \mathbf{e}_{\theta}-B_{\mathrm{FL}}^{\varphi} \cos \varphi \mathbf{e}_{\varphi},
\end{gathered}
$$

where $\mathbf{e}_{\theta}$ and $\mathbf{e}_{\varphi}$ are the polar and azimuthal unit vectors, respectively, and $B_{D L, F L}^{\theta}$ and $B_{\mathrm{DL}, \mathrm{FL}}^{\varphi}$ are functions of the magnetization orientation, defined by the angles $\theta$ and $\varphi$ [see Fig. 17(b)]. In polycrystalline bilayers with $\mathrm{C}_{2 v}$ symmetry, the angular dependence of the polar components simplifies to a Fourier series expansion of the type $B_{\mathrm{DL}, \mathrm{FL}}^{\theta}=B_{\mathrm{DL}, \mathrm{FL}}^{\{0\}}+B_{\mathrm{DL}, \mathrm{FL}}^{\{2\}} \sin ^{2} \theta+B_{\mathrm{DL}, \mathrm{FL}}^{\{4\}} \sin ^{4} \theta+\ldots$ The azimuthal components, on the other hand, are found by experiments to be only weakly angle-dependent and are approximated by $B_{\mathrm{DL}, \mathrm{FL}}^{\varphi} \approx B_{\mathrm{DL}, \mathrm{FL}}^{\{0\}}$ (Garello et al., 2013).

\section{B. Measurement techniques}

Experimental measurements of SOT rely on probing the effect of the electric current on the orientation of the magnetization, e.g., by inducing resonant and nonresonant oscillations, switching, or DW motion. Schematically, one must first determine the magnetization angle as a function of the amplitude and phase of the applied current and, second, extract the effective magnetic fields that are responsible for the observed dynamics. In electrical and optical measurements, the magnetization dynamics is detected through changes of the transverse or longitudinal conductivity, which are mainly due to the anomalous Hall effect (AHE) and anisotropic magnetoresistance (AMR), but include also the linear SMR (Kim et al., 2016; Nakayama et al., 2013), linear Rashba magnetoresistance (Kobs et al., 2011; Nakayama et al., 2016), as well as nonlinear magnetoresistance terms proportional to the current-induced spin accumulation (Avci et al., 2015a,b; Olejník et al., 2015; Yasuda et al., 2017, 2016). Further, current injection always results in magnetothermal effects due to the thermal gradients induced by Joule heating and asymmetric heat dissipation (Avci et al., 2014a), which affect the conductivity proportionally to $j_{c}^{2}$. The thermal gradients that develop along $\left(\nabla_{x} T\right)$ or perpendicular to the magnetic layer $\left(\nabla_{z} T\right)$ contribute to the conductivity through the anomalous Nernst effect (ANE) and, to a smaller extent, through the spin Seebeck effect and the inverse spin Nernst effect. The direction of the induced voltage is $\sim \nabla T \times \mathbf{m}$, a

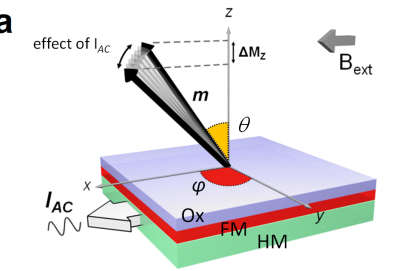

b
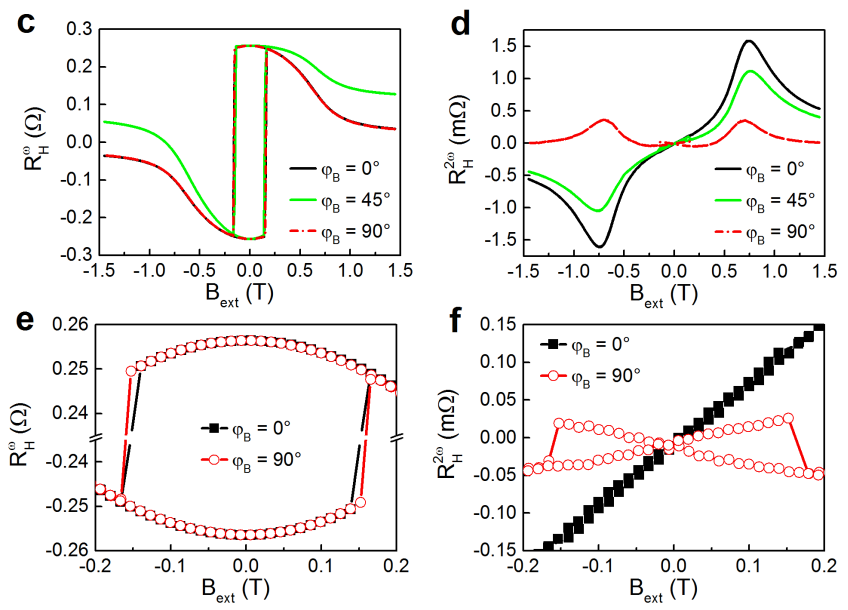

FIG. 18 (Color online) (a) Schematic of the effect of an ac current on the magnetization and (b) experimental setup for HHV measurements. (c) $R_{x y}^{\omega}$ and (d) $R_{x y}^{2 \omega}$ of a $\mathrm{Pt}(5 \mathrm{~nm}) / \mathrm{Co}(1 \mathrm{~nm}) / \mathrm{AlO}_{x}$ layer measured with a sinusoidal current of amplitude $j_{c}=10^{7} \mathrm{~A} / \mathrm{cm}^{2}$ and external magnetic field applied at $\varphi_{B}=0^{\circ}, 45^{\circ}$, and $90^{\circ}$. (e,f) Close up of the curves in (c,d) showing the field range where the small angle approximation can be applied (Baumgartner, 2018).

which modifies both the longitudinal $\left(\sim m_{y}\right)$ and transverse conductivities $\left(\sim m_{x}\right)$. The relative influence of the above effects on SOT measurements depends on the system under investigation and experimental technique. The AMR, AHE, and ANE usually dominate the magnetization dependence of the conductivity and can be properly separated owing to their different symmetry and magnetic field dependence (Avci et al., 2014a; Garello et al., 2013) or frequency-dependent optical response (Fan et al., 2016; Montazeri et al., 2015). In the following, we describe the three main techniques used to characterize the SOT measurements: harmonic Hall voltage analysis (HHV), spin-torque ferromagnetic resonance (ST-FMR), and magneto-optical Kerr effect (MOKE). Less precise SOT estimates can be obtained from magnetization switching and DW displacements, which are discussed separately in Sections IV.E and VI.

\section{Harmonic Hall voltage analysis}

This method detects the harmonic response of the magnetization to a low frequency ac current, typically up to a few $\mathrm{kHz}$. Originally, this approach was developed by assuming the simplest form of FL torque (Pi et al., 
2010) and neglecting the DL torque and the transverse AMR (the planar Hall effect, PHE). It was soon extended to both components of the torques accounting for both the AHE and PHE (Garello et al., 2013; Hayashi et al., 2014; Kim et al., 2013a), as well as for the torque angular dependence (Garello et al., 2013; Qiu et al., 2014) and magnetothermal effects (Avci et al., 2014a; Ghosh et al., 2017). SOT measurements are performed by analyzing the second HHV that arises due to the homodyne mixing of the ac current with the Hall resistance modulated by the oscillations of the magnetization induced by the SOTs [Fig. 18(a)]. Since the magnetization dynamics is much faster than the current frequency $\omega$, the magnetization is assumed to be in quasi-static equilibrium at all times, at a position determined by the sum of the anisotropy field, external magnetic field, and currentinduced fields. To first order in the current, the timedependent Hall resistance $R_{x y}(t)$ is given by

$$
R_{x y}\left(\mathbf{B}_{\text {ext }}+\mathbf{B}_{I}(t)\right) \approx R_{x y}\left(\mathbf{B}_{\text {ext }}\right)+\frac{d R_{x y}}{d \mathbf{B}_{I}} \cdot \mathbf{B}_{I} \sin (\omega t),
$$

where $\mathbf{B}_{\text {ext }}$ is the external magnetic field and $\mathbf{B}_{I}=$ $\mathbf{B}_{\mathrm{DL}}+\mathbf{B}_{\mathrm{FL}}+\mathbf{B}^{O e}$ is the effective current-induced field due to the sum of the DL and FL SOT and the Oersted field. The Hall voltage $V_{x y}(t)=R_{x y}(t) I_{0} \sin (\omega t)$ then reads

$$
V_{x y}(t) \approx I_{0}\left[R_{x y}^{0}+R_{x y}^{\omega} \sin (\omega t)+R_{x y}^{2 \omega} \cos (2 \omega t)\right],
$$

where $I_{0}$ is the current amplitude, $R_{x y}^{0}=\frac{1}{2} \frac{d R_{x y}}{d B_{I}} \cdot B_{I}$, $R_{x y}^{\omega}=R_{x y}\left(B_{\text {ext }}\right)$, and $R_{x y}^{2 \omega}=-\frac{1}{2} \frac{d R_{\omega}}{d B_{I}} \cdot B_{I}+R_{\nabla T}^{2 \omega}$ are the zero, first, and second harmonic components of $R_{x y}$, respectively. The first harmonic term, shown in Fig. 18(c) as a function of external field, is analogous to the dc Hall resistance and given by

$$
R_{x y}^{\omega}=R_{\mathrm{AHE}} \cos \theta+R_{\mathrm{PHE}} \sin ^{2} \theta \sin (2 \varphi),
$$

where $R_{\mathrm{AHE}}$ and $R_{\mathrm{PHE}}$ are the anomalous and planar Hall coefficients. This term serves two purposes, namely to determine the polar angle of the magnetization using Eq. (51) when $\varphi=0^{\circ}, 90^{\circ}$ and to measure the susceptibility of the magnetization to the magnetic field, providing self-calibration to the SOT measurement. The second harmonic term includes the SOT modulation of the Hall resistance as well as an extra contribution due to Joule heating, $R_{\nabla T}^{2 \omega}$. In general, the two contributions may have a comparable magnitude and must be separated by either symmetry or magnetic field dependent measurements (Avci et al., 2014a; Ghosh et al., 2017). Assuming that $R_{\nabla T}^{2 \omega}$ is negligible or has been subtracted from $R_{x y}^{2 \omega}$, it is straightforward to show that

$$
R_{x y}^{2 \omega}=A_{\theta} \mathbf{B}_{I} \cdot \mathbf{e}_{\theta}+A_{\varphi} \mathbf{B}_{I} \cdot \mathbf{e}_{\varphi},
$$

where $A_{\theta}=\frac{d R_{x y}^{\omega}}{d B_{\text {ext }}}\left[I_{0} \sin \left(\theta_{B}-\theta\right)\right]^{-1}$ and $A_{\varphi}=$ $R_{\mathrm{PHE}} \sin ^{2} \theta \frac{d \sin (2 \varphi)}{d \varphi}\left[I_{0} \sin \theta_{B} \cos \left(\varphi_{B}-\varphi\right) B_{\text {ext }}\right]^{-1}$. Here $\theta_{B}$ and $\varphi_{B}$ are the polar and azimuthal angles of the applied magnetic field. Equation (52) allows one to find the polar and azimuthal components of $B_{\mathrm{DL}}$ and $B_{\mathrm{FL}}$ as a function of the magnetization angle by measuring the dependence of $R_{x y}^{2 \omega}$ on $B_{\text {ext }}$. Figure 18(d) shows an example of $R_{x y}^{2 \omega}$ measured at $\varphi_{B}=0^{\circ}$ and $\varphi_{B}=90^{\circ}$. These curves are, respectively, odd and even with respect to magnetization reversal, reflecting the different symmetry of $B_{\mathrm{DL}}$ and $B_{\mathrm{FL}}$ (Garello et al., 2013). Because the DL torque is larger when $\mathbf{m}$ lies in the $x z$ plane, whereas the FL torque tends to align $\mathbf{m}$ towards $y$, measurements taken at $\varphi_{B}=0^{\circ}\left(\varphi_{B}=90^{\circ}\right)$ reflect the character of the DL (FL) effective fields. In general, four independent measurements at different azimuthal angles are required to determine the four effective field components in Eqs. $(47,48)$.

In uniaxial and easy plane systems the number of independent measurements can be reduced to two, typically at $\varphi_{B}=0, \frac{\pi}{2}$ or $\varphi_{B}=\frac{\pi}{4}, \frac{3 \pi}{4}$ (Avci et al., 2014a; Garello et al., 2013). A further simplification is achieved using the small angle approximation, which is valid for perpendicularly magnetized samples when the magnetization deviates by at most a few degrees from the $z$-axis (Hayashi et al., 2014; Kim et al., 2013a). In this case, $R_{x y}^{2 \omega}$ varies linearly with the external field, as shown in Fig. 18(f) and the SOTs are extracted by performing two sets of measurement at $\varphi_{B}=0$ and $\frac{\pi}{2}$ :

$$
\begin{aligned}
B_{\mathrm{DL}} & =-\frac{2}{1-4 r^{2}}\left(b_{x}+2 r b_{y}\right), \\
B_{\mathrm{FL}} & =-\frac{2}{1-4 r^{2}}\left(b_{y}-2 r b_{x}\right),
\end{aligned}
$$

where $b_{i}=\frac{\partial R_{x y}^{2 \omega}}{\partial B_{\text {ext }}} / \frac{\partial^{2} R_{x y}^{\omega}}{\partial B_{\text {ext }}^{2}}$ is measured for $B_{\text {ext }} \| i=x, y$ and the partial derivatives are calculated by linear fits of the curves shown in Fig. 18(f). This approximation provides only the lowest order contribution to the SOT. However, because of its simple implementation, it is widely used for characterizing the SOTs in systems with perpendicular anisotropy. HHV measurements can also be generalized to angular scans of the magnetization at constant external field, which is particularly suited for in-plane magnetized samples (Avci et al., 2014a), thus providing a versatile and sensitive method to characterize the SOT in a variety of systems.

\section{Spin-torque ferromagnetic resonance}

This method consists in exciting the magnetization of the FM using a radio-frequency $(\mathrm{RF})$ charge current. The magnetization of the sample is excited through the spin torque and exhibits FMR when varying either the applied magnetic field or the current magnitude. This concept was initially developed in the context of spin-valves (Sankey et al., 2006) and MTJs (Kubota et al., 2007; 
a
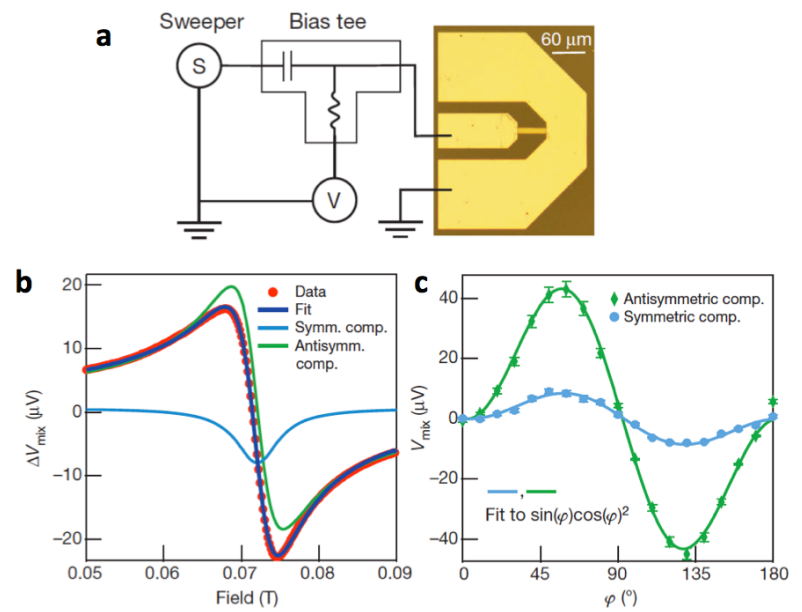

FIG. 19 (Color online) (a) Schematic of the circuit used for the ST-FMR measurement and the sample contact geometry. (b) Measured ST-FMR at room temperature with microwave frequency $\omega / 2 \pi=58 \mathrm{GHz}$ for $\mathrm{Bi}_{2} \mathrm{Se}_{3}(8 \mathrm{~nm}) / \mathrm{Ni}_{80} \mathrm{Fe}_{20}(16 \mathrm{~nm})$. A fixed microwave power of $5 \mathrm{dBm}$ is absorbed by the device $\left(I_{\mathrm{RF}}=7.7 \pm 1.1 \mathrm{~mA}\right)$ and $B$ is oriented at an angle $\varphi=\pi / 4$ from the current direction. The lines are fits to Eq. (55) showing the symmetric and antisymmetric resonance components. (c) Measured dependence on the magnetic field angle $\varphi$ for the symmetric and antisymmetric resonance components for a different sample. Adapted from (Mellnik et al., 2014).

Sankey et al., 2007; Tulapurkar et al., 2005) and more recently extended to the case of ultrathin magnetic bilayers (Liu et al., 2011, 2012b) and bulk non-centrosymmetric magnetic semiconductors (Fang et al., 2011; Kurebayashi et al., 2014).

The dc voltage that develops across the sample [Fig. 19(a)] arises from the mixing of the RF current and the RF AMR due to the oscillating magnetization. It corresponds to the zero harmonic component in Eq. (50) and here is strongly amplified due to the resonant magnetization dynamics. This rectified voltage gives information on the physical parameters of the magnetic material as well as on the nature of the torques that drive the excitation. In the context of an in-plane system with AMR driven by SOTs, the mixing voltage reads (Liu et al., 2011; Reynolds et al., 2017)

$$
\begin{aligned}
& V_{\mathrm{mix}}=-\frac{\gamma}{2} I_{\mathrm{RF}} \partial_{\varphi} R \cos \varphi_{B}\left[\tau_{\mathrm{DL}} F_{S}(B)+\tau_{\mathrm{FL}} F_{A}(B)\right](, 55) \\
& F_{S}(B)=\frac{\alpha \omega^{2}\left(2 B+\mu_{0} M_{\mathrm{s}}\right)}{\left(\omega^{2}-\omega_{0}^{2}\right)^{2}+\alpha^{2} \gamma^{2} \omega^{2}\left(2 B+\mu_{0} M_{\mathrm{s}}\right)}, \\
& F_{A}(B)=\frac{\gamma^{2} B\left(2 B+\mu_{0} M_{\mathrm{s}}\right)^{2}-\alpha \omega^{2}\left(2 B+\mu_{0} M_{\mathrm{s}}\right)}{\left(\omega^{2}-\omega_{0}^{2}\right)^{2}+\alpha^{2} \gamma^{2} \omega^{2}\left(2 B+\mu_{0} M_{\mathrm{s}}\right)},
\end{aligned}
$$

where $\omega$ is the frequency of the RF current $I_{\mathrm{RF}}$ and $\omega_{0}=$ $\gamma \sqrt{B\left(B+\mu_{0} M_{\mathrm{s}}\right)}$ is the resonance frequency. The first contribution has a symmetric Lorentzian shape $\left(\sim F_{S}\right)$ that is directly proportional to the DL torque, while the second has an antisymmetric shape $\left(\sim F_{A}\right)$, providing information about the FL torque (including the Oersted field torque). A picture of the experimental apparatus is given in Fig. 19(a), together with the field-dependent and angular-dependent mixing voltages in Figs. 19(b,c), respectively. This method is used extensively to probe torques in magnetic bilayers with in-plane magnetization, as well as in non-centrosymmetric bulk magnets, as explained in Section V. This effect is the reciprocal to spin pumping, where the field-excited precessing magnetization pumps a spin current in the adjacent NM (Saitoh et al., 2006; Tserkovnyak et al., 2002a).

Similar to other techniques, applying this method to ultrathin bilayer systems requires extreme care. First, the amplitude of the RF current generating the torques needs to be calibrated precisely using a network analyzer. Such a calibration might require thickness-dependent measurements to characterize possible size-dependent effects (Nguyen et al., 2016). Second, Eqs. 55 only account for the rectification arising from AMR, but other sources such as spin Hall magnetoresistance (SMR) can also contribute to the mixing voltage (Nakayama et al., 2013), which should be properly accounted for (Wang et al., 2016b; Zhang et al., 2016d). Third, the phase difference between the RF current and the RF field can also have significant impact on the output signal (Harder et al., 2011). We refer the interested reader to the specialized literature for more information (Harder et al., 2016). A fourth issue is that this method assumes the simplest form of the torque, Eq. (1), neglecting the angular dependence of SOT (Garello et al., 2013).

\section{Magneto-optic Kerr effect}

The magneto-optic Kerr effect (MOKE) allows for detecting the in-plane and out-of-plane components of the magnetization through the rotation of the light polarization upon reflection from a magnetic surface (Qiu and Bader, 2000). MOKE microscopy, with a wavelengthlimited resolution of about $1 \mu \mathrm{m}$, has been used extensively to characterize SOT-induced domain nucleation and displacement (Emori et al., 2013; Miron et al., 2010; Ryu et al., 2013; Safeer et al., 2016) as well as the currentinduced spin accumulation in bare $\mathrm{Pt}$ and $\mathrm{W}$ layers (Stamm et al., 2017). MOKE-based detection schemes have been used also to estimate the SOT amplitude by measuring the oscillations of the magnetization induced by an ac current in thin metal bilayers (Fan et al., 2014a).

Vector measurements of the SOT are based on the separate calibration of the first- and second-order magnetooptic coefficients, $f_{\perp}$ and $f_{\|}$, which parameterize the coupling of the light to the out-of-plane and in-plane magnetization, respectively (Fan et al., 2016; Montazeri et al., 2015). Such a technique measures the DL and FL components of the SOT as a function of the magnetization angle via the polar and quadratic MOKE response, respectively, using only normally incident light [see Fig. 20(a)]. 

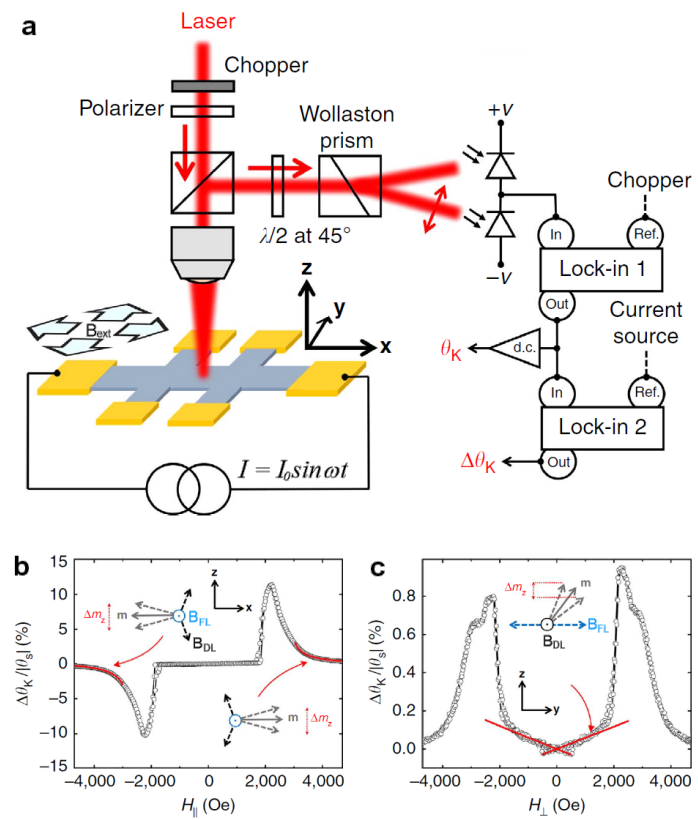

FIG. 20 (Color online) (a) Schematic of a MOKE setup for SOT detection. (b,c) Differential Kerr angle $\Delta \theta_{K}$ measured on a $\mathrm{Ta}(5 \mathrm{~nm}) / \mathrm{CoFeB}(1.1 \mathrm{~nm}) / \mathrm{MgO}(2.0 \mathrm{~nm})$ trilayer with perpendicular magnetic anisotropy for $\mathbf{B}_{\text {ext }} \| \mathbf{j}_{c}$ (b) and $\mathbf{B}_{\text {ext }} \perp \mathbf{j}_{c}$ (c) with current amplitude $j_{c}=4.6 \times 10^{6} \mathrm{Acm}^{-2}$. Adapted from Montazeri et al. (2015).

Similar to the Hall resistance (Eq. 49), the Kerr rotation measured during ac current injection can be Taylor expanded as

$$
\theta_{K}\left(\mathbf{B}_{\mathrm{ext}}+\mathbf{B}_{I}(t)\right) \approx \theta_{K}\left(\mathbf{B}_{\mathrm{ext}}\right)+\frac{d \theta_{K}}{d \mathbf{B}_{I}} \cdot \mathbf{B}_{I} \sin (\omega t) .
$$

Here, the first term is the equilibrium Kerr angle given by $\theta_{K}=f_{\perp} m_{z}+f_{\|}\left[1 / 2\left(m_{y}^{2}-m_{x}^{2}\right) \sin 2 \phi_{p}+m_{x} m_{y} \cos 2 \phi_{p}\right]$, with $\phi_{p}$ the angle between the light polarization and $\mathbf{B}_{e x t}$, and the second term results in the differential Kerr signal $\Delta \theta_{K}=\left(d \theta_{K} / d I\right) I_{0}$ due to the currentinduced fields. In analogy with the HHV technique, measurements of $\Delta \theta_{K}$ are mostly sensitive to changes of $m_{z}$. Thus, measurements taken with $\mathbf{B}_{\text {ext }} \| \mathbf{x}$ reflect the strength of the DL effective field,

$$
\Delta \theta_{K}=\frac{f_{\perp} B_{D L}}{B_{e x t}-B_{K}}+\frac{f_{\|} \cos 2 \phi_{p}\left(B_{F L}+B_{O e}\right)}{B_{e x t}},
$$

where $B_{K}$ is the magnetic anisotropy field and $f_{\|} \ll f_{\perp}$. Conversely, measurements taken with $\mathbf{B}_{\text {ext }} \| \mathbf{y}$ reflect the strength of the FL effective field,

$$
B_{F L}=\frac{-2 \partial\left(\Delta \theta_{K}\right) / \partial B_{e x t}}{\partial^{2} \theta_{K} / \partial B_{e x t}^{2}} .
$$

Figure 20(b) shows that $\Delta \theta_{K}$ exhibits an antisymmetric (symmetric) line shape consistent with the symmetry of $B_{D L}\left(B_{F L}\right)$ under magnetization reversal, in close analogy with $R_{x y}^{2 \omega}$ [Fig. 18(d)]. SOT vector measurements
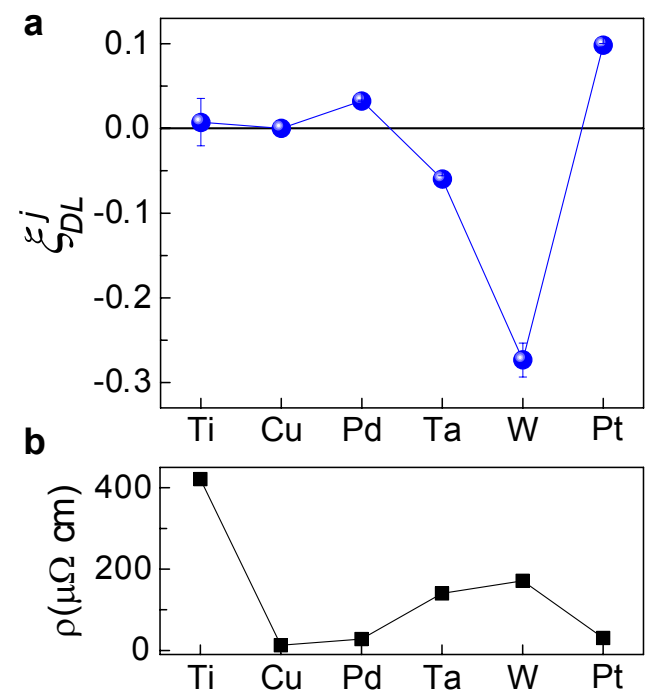

FIG. 21 (Color online) (a) DL SOT efficiency in $\mathrm{X}(8$ $\mathrm{nm}) / \mathrm{Co} / \mathrm{AlO}_{x}(2)$ trilayers, where $\mathrm{X}=\mathrm{Ti}, \mathrm{Cu}, \mathrm{Pd}$, Ta, W, Pt. The data are measured using the HHV method. The Co thickness is $2.5 \mathrm{~nm}$ except for the Pd sample where it is 0.6 $\mathrm{nm}$. (b) Room temperature resistivity of the NM. Adapted from Avci et al. (2015b); Ghosh et al. (2017).

performed by MOKE agree well with HHV (Montazeri et al., 2015) and ST-FMR measurements (Fan et al., 2016) and can be used to characterize the SOT in metallic as well as insulating FMs. An advantage of this technique is that it is less sensitive to thermoelectric and inductive effects compared to all-electrical SOT probes, and that it offers spatial resolution comparable to the wavelength of the probing laser beam.

\section{Materials survey}

\section{Metal multilayers}

The most studied SOT systems are composed of a metallic FM deposited on a NM layer, often capped by an amorphous or crystalline oxide layer. These systems present strong DL and FL SOTs, of the order of a few $\mathrm{mT}$ per $10^{7} \mathrm{~A} / \mathrm{cm}^{2}\left(\xi^{j} \approx 0.1\right.$, see Table II), are easy to fabricate, and compatible with established processing of magnetic materials for memory applications. An early experimental observation of the DL SOT in FM metals was reported by Ando et al. (2008) in a Pt/NiFe bilayer resonantly excited by an external microwave field, by measuring the change of magnetic damping upon injection of a dc current. This effect was attributed to the SHE of the Pt layer and later extended to the excitation of FMR upon injection of an RF current (Liu et al., 2011). Evidence for the FL SOT was first reported by Miron et al. (2010) by observing that the current-induced nucleation of magnetic domains in perpendicularly magnetized $\mathrm{Pt} / \mathrm{Co} / \mathrm{AlO}_{x}$ wires is either enhanced or quenched by ap- 


\begin{tabular}{|c|c|c|c|c|c|c|c|c|c|}
\hline Structure & & MA & Method & $B_{\mathrm{DL}} / j$ & $B_{\mathrm{FL}} / j$ & $\xi_{\mathrm{DL}}^{j}$ & $\xi_{\mathrm{FL}}^{j}$ & $\xi_{\mathrm{DL}}^{E}$ & $\xi_{\mathrm{FL}}^{E}$ \\
\hline \multicolumn{10}{|l|}{ Nonmagnetic metals } \\
\hline $\mathrm{Pt}(3) / \mathrm{Co}(0.6) / \mathrm{AlO}_{x}(1.6)$ & (Garello et al., 2013) & $\mathrm{OP}$ & $\mathrm{HHV}$ & -6.9 & 4 & 0.13 & -0.073 & 3.5 & -2.0 \\
\hline $\mathrm{Pt}(3) / \mathrm{CoFe}(0.6) / \mathrm{MgO}(1.8)$ & (Emori et al., 2013) & $\mathrm{OP}$ & HHV & -5 & 2 & 0.064 & -0.024 & & \\
\hline $\mathrm{Ti}(1) / \mathrm{CoFe}(0.6) / \operatorname{Pt}(5)$ & (Fan et al., 2014a) & IP & MOKE & 3.2 & -0.3 & 0.074 & -0.008 & & \\
\hline $\mathrm{Pt}(5) / \mathrm{Co}(1) / \mathrm{MgO}(2)$ & (Nguyen et al., 2016) & $\mathrm{OP}$ & $\mathrm{HHV}$ & -4.5 & 1 & 0.11 & -0.024 & 2.43 & -0.53 \\
\hline $\mathrm{Pt}(5) / \mathrm{Ni}_{80} \mathrm{Fe}_{20}(8) / \mathrm{AlO}_{x}(2)$ & (Fan et al., 2016) & IP & MOKE & -0.49 & 0.71 & 0.082 & -0.12 & 2.64 & -3.88 \\
\hline $\mathrm{YIG}(50) / \mathrm{Pt}(4)$ & (Montazeri et al., 2015) & IP & MOKE & 0.29 & & 0.03 & & & \\
\hline $\operatorname{TmIG}(8) / \operatorname{Pt}(5)$ & (Avci et al., 2017) & $\mathrm{OP}$ & $\mathrm{HHV}$ & 0.59 & & 0.014 & & & \\
\hline $\mathrm{Ta}(4) / \mathrm{CoFeB}(1.1) / \mathrm{MgO}(1.6)$ & (Liu et al., 2012b) & $\mathrm{OP}$ & $\mathrm{HHV}$ & 3.5 & & -0.13 & & -0.68 & \\
\hline $\mathrm{Ta}(3) / \mathrm{CoFeB}(0.9) / \mathrm{MgO}(2)$ & (Avci et al., 2014b) & $\mathrm{OP}$ & HHV & 3.2 & -2.1 & -0.06 & 0.04 & -0.34 & 0.22 \\
\hline $\mathrm{Ta}(3) / \mathrm{CoFeB}(0.9) / \mathrm{MgO}(2)^{a}$ & (Garello et al., 2013) & $\mathrm{OP}$ & $\mathrm{HHV}$ & 2.4 & -4.5 & -0.07 & 0.12 & -0.36 & 0.67 \\
\hline $\mathrm{Ta}(1.5) / \mathrm{CoFeB}(1) / \mathrm{MgO}(2)^{a}$ & (Kim et al., 2013a) & $\mathrm{OP}$ & $\mathrm{HHV}$ & 1.35 & -4.46 & -0.03 & 0.11 & -0.14 & 0.48 \\
\hline $\mathrm{Ta}(2) / \mathrm{CoFeB}(0.8) / \mathrm{MgO}(2)^{a}$ & (Qiu et al., 2014) & $\mathrm{OP}$ & $\mathrm{HHV}$ & 4.4 & -19.4 & -0.11 & 0.47 & & \\
\hline $\mathrm{Ta}(5) / \mathrm{CoFeB}(1.1) / \mathrm{MgO}(2)^{a}$ & (Montazeri et al., 2015) & $\mathrm{OP}$ & MOKE & 2.0 & -3.3 & -0.05 & 0.08 & & \\
\hline $\mathrm{W}(5) / \operatorname{CoFeB}(0.85) / \operatorname{Ti}(1)^{a}$ & (Pai et al., 2012) & IP & ST-FMR & & & -0.33 & & & \\
\hline $\mathrm{Hf}(3.5) / \mathrm{CoFeB}(1) / \mathrm{MgO}(2)^{a}$ & (Torrejon et al., 2014) & $\mathrm{OP}$ & $\mathrm{HHV}$ & 0.8 & -2.6 & -0.02 & 0.06 & & \\
\hline $\mathrm{Hf}(3.5) / \mathrm{CoFeB}(1.1) / \mathrm{MgO}(2)^{a}$ & (Akyol et al., 2016) & $\mathrm{OP}$ & $\mathrm{HHV}$ & 5 & & -0.17 & & & \\
\hline $\mathrm{Hf}(10) / \mathrm{CoFeB}(1.1) / \mathrm{MgO}(2)^{a}$ & (Akyol et al., 2016) & $\mathrm{OP}$ & $\mathrm{HHV}$ & -1 & & 0.03 & & & \\
\hline $\mathrm{Hf}(1) / \mathrm{CoFeB}(1) / \mathrm{MgO}(2)$ & (Ramaswamy et al., 2016) & $\mathrm{OP}$ & $\mathrm{HHV}$ & -0.24 & 0.9 & 0.007 & -0.03 & & \\
\hline $\mathrm{Hf}(6) / \mathrm{CoFeB}(1) / \mathrm{MgO}(2)$ & (Ramaswamy et al., 2016) & OP & $\mathrm{HHV}$ & 9 & -27 & -0.28 & 0.82 & & \\
\hline $\mathrm{Pd}(7) / \mathrm{Co}(0.6) / \mathrm{AlO}_{x}(1.6)$ & (Ghosh et al., 2017) & $\mathrm{OP}$ & $\mathrm{HHV}$ & -1.3 & 0.7 & 0.03 & -0.015 & 1.0 & -0.55 \\
\hline \multicolumn{10}{|l|}{ Oxidized metals } \\
\hline $\mathrm{WO}_{x}(6) / \mathrm{CoFeB}(6) / \mathrm{TaN}(2)$ & (Demasius et al., 2016) & IP & ST-FMR & & & -0.49 & & & \\
\hline $\mathrm{SiO}_{2} / \mathrm{Ni}_{80} \mathrm{Fe}_{20}(8) / \mathrm{CuO}_{x}(10)$ & (An et al., 2016) & IP & ST-FMR & & & 0.08 & -0.08 & & \\
\hline $\mathrm{Ti}(1.2) / \mathrm{Ni}_{80} \mathrm{Fe}_{20}(1.5) / \mathrm{AlO}_{x}(1.5)$ & (Emori et al., 2016) & IP & ST-FMR & & 0.15 & & -0.01 & & \\
\hline \multicolumn{10}{|c|}{ Metal alloys } \\
\hline $\mathrm{CuAu}(8) / \mathrm{Ni}_{80} \mathrm{Fe}_{20}(1.5)$ & (Wen et al., 2017) & IP & HHV & -1.9 & 0.58 & 0.01 & -0.003 & 0.33 & -0.1 \\
\hline $\mathrm{Ni}_{80} \mathrm{Fe}_{20}(9) / \mathrm{Ag}(2) / \mathrm{Bi}(4)$ & (Jungfleisch et al., 2016) & IP & ST-FMR & & & 0.18 & $0.14^{b, c}$ & & \\
\hline \multicolumn{10}{|l|}{ Antiferromagnets } \\
\hline $\operatorname{IrMn}(8) / \mathrm{Ni}_{80} \mathrm{Fe}_{20}(4) / \mathrm{Al}(2)$ & (Tshitoyan et al., 2015) & IP & ST-FMR & -2.2 & -1.7 & 0.22 & 0.17 & & \\
\hline $\operatorname{IrMn}_{3}[001](6) / \mathrm{Ni}_{80} \mathrm{Fe}_{20}(6) / \mathrm{TaN}$ & (Zhang et al., 2016b) & IP & ST-FMR & & & 0.20 & & & \\
\hline $\operatorname{IrMn}_{3}[111](6) / \mathrm{Ni}_{80} \mathrm{Fe}_{20}(6) / \mathrm{TaN}$ & (Zhang et al., 2016b) & IP & ST-FMR & & & 0.12 & & & \\
\hline $\operatorname{IrMn}_{3}(5) / \mathrm{CoFeB}(1) / \mathrm{MgO}^{a}$ & (Wu et al., 2016) & OP & $\mathrm{HHV}$ & -1.8 & 0.7 & 0.06 & -0.02 & & \\
\hline $\operatorname{PtMn}(8) / \mathrm{Co}(1) / \mathrm{MgO}(1.6)$ & (Ou et al., 2016) & IP & ST-FMR & & & 0.16 & -0.04 & & \\
\hline $\operatorname{MgO}(1.6) / \mathrm{Co}(1) / \operatorname{PtMn}(8)$ & (Ou et al., 2016) & IP & ST-FMR & & & 0.19 & $\simeq 0$ & & \\
\hline \multicolumn{10}{|l|}{ Semiconductors and semimetals } \\
\hline$(\mathrm{Ga}, \mathrm{Mn}) \mathrm{As}(20) / \mathrm{Fe}(2) / \mathrm{Al}(2)$ & (Skinner et al., 2015) & IP & ST-FMR & $-0.34^{b}$ & $0.26^{c, d}$ & $0.03^{b}$ & $-0.02^{c, d}$ & & \\
\hline $\operatorname{MoS}_{2}(0.8) / \mathrm{CoFeB}(3) / \mathrm{TaO}_{x}(3)$ & (Shao et al., 2016) & IP & $\mathrm{HHV}$ & $\simeq 0$ & $\sim 0.008$ & $\simeq 0$ & -0.14 & $\simeq 0$ & -0.03 \\
\hline $\mathrm{WSe}_{2}(0.8) / \mathrm{CoFeB}(3) / \mathrm{TaO}_{x}(3)$ & (Shao et al., 2016) & IP & $\mathrm{HHV}$ & $\simeq 0$ & 0.012 & $\simeq 0$ & $\sim-0.3$ & $\simeq 0$ & -0.06 \\
\hline $\mathrm{WTe}_{2} / \mathrm{Ni}_{80} \mathrm{Fe}_{20}(6) / \mathrm{Al}(1)$ & (MacNeill et al., 2017) & IP & ST-FMR & & & $0.04^{b}$ & & $0.12^{b}$ & $0.09^{c}$ \\
\hline \multicolumn{10}{|l|}{ Topological insulators } \\
\hline $\mathrm{Bi}_{2} \mathrm{Se}_{3}(8) / \mathrm{Ni}_{80} \mathrm{Fe}_{20}(16) / \mathrm{Al}(2)$ & (Mellnik et al., 2014) & IP & ST-FMR & $-1 \cdot 10^{-4}$ & $-2 \cdot 10^{-4}$ & 1.9 & 2.5 & 1.1 & 1.4 \\
\hline $\mathrm{Bi}_{2} \mathrm{Se}_{3}(20) / \mathrm{CoFeB}(5) / \mathrm{MgO}(2)$ & (Wang et al., 2015) & IP & ST-FMR & & & $0.08^{b}$ & $0.05^{b}$ & & \\
\hline $\mathrm{Bi}_{2} \mathrm{Se}_{3}(7.4) / \mathrm{CoTb}(4.6) / \mathrm{SiN}_{x}(3)$ & (Han et al., 2017) & OP & Switching & -6.1 & & 0.16 & & & \\
\hline$(\mathrm{Bi}, \mathrm{Sb})_{2} \mathrm{Te}_{3}(8) / \mathrm{CoTb}(8) / \mathrm{SiN}_{x}(3)$ & (Han et al., 2017) & $\mathrm{OP}$ & Switching & -8 & & 0.4 & & & \\
\hline
\end{tabular}

${ }^{a}$ Annealed. ${ }^{b}$ Average value. ${ }^{c}$ Sign uncertain.

TABLE II SOTs in magnetic multilayers. The thickness of the layers is given in $\mathrm{nm}$ with the topmost layer on the right. The following units are used for the effective fields and SOT efficiencies: $B_{\mathrm{DL}, \mathrm{FL}} / j\left[\mathrm{mT} /\left(10^{7} \mathrm{~A} / \mathrm{cm}^{2}\right)\right]$, $\xi_{\mathrm{DL}, \mathrm{FL}}^{j}[$ adimensional], and $\xi_{\mathrm{DL}, \mathrm{FL}}^{E}\left[(\hbar / 2 e) 10^{5}(\Omega m)^{-1}\right]$. The sign of $B_{\mathrm{DL}}$ and $B_{\mathrm{FL}}$ is defined as in Eqs. $(47,48)$. $\xi_{\mathrm{DL}}>0$ corresponds to the same sign of the DL torque as for Pt, whereas $\xi_{\mathrm{FL}}<0$ indicates that $B_{\mathrm{FL}}$ is opposite to the Oersted field. The magnetic anisotropy (MA) of the FM layers is indicated as out-of-plane (OP) or in-plane (IP). The values for the OP samples are given for the magnetization lying close to the easy axis. All measurements have been carried out at room temperature. 
plying an in-plane magnetic field at an angle of $\pm 90^{\circ}$ relative to the current. This effect was attributed to the action of a Rashba-like effective field and later quantitatively estimated by HHV measurements (Garello et al., 2013; Pi et al., 2010).

A major breakthrough was achieved in 2011 when bipolar magnetization switching was demonstrated in perpendicular $\mathrm{Pt} / \mathrm{Co} / \mathrm{AlO}_{x}$ dots (Miron et al., 2011a), establishing the relevance of SOT for applications. Miron et al. observed that the symmetry of the switching field corresponds to a DL torque consistent with either the SHE or the iSGE, and argued that the SHE of Pt alone could not account for the magnitude of the torque. Other experiments favored a SHE-only explanation of the switching mechanism (Liu et al., 2012a), triggering an ongoing debate on the origin of the torques (see Section IV.C.5). These experiments were rapidly followed by measurements of SOTs and magnetization switching in $\mathrm{Ta} / \mathrm{CoFeB} / \mathrm{MgO}$ (Avci et al., 2014b; Emori et al., 2013; Garello et al., 2013; Kim et al., 2013a; Liu et al., 2012b) and $\mathrm{W} / \mathrm{CoFeB} / \mathrm{MgO}$ layers (Pai et al., 2012), which showed that the DL SOT correlates with the sign of the spin-orbit coupling constant and the SHE of the NM layer, whereas the FL torque has a more erratic behavior depending on the type of FM and interface structure (Pai et al., 2015).

The largest SOT efficiencies are found in the $5 d$ metals, in particular for the highly resistive $\beta$-phase of $\mathrm{W}$ and Ta as well for fcc Pt (Fig. 21). In metals where the spin Hall conductivity $\sigma_{\mathrm{sh}}$ is of intrinsic origin, the spin Hall angle is directly proportional to the longitudinal resistivity, given by $\theta_{\mathrm{sh}}=\sigma_{\mathrm{sh}} \rho$. Pt and $\mathrm{Pd}$ display a large SOT efficiency despite their moderate resistivity (Ghosh et al., 2017; Nguyen et al., 2016), which is attributed to their large intrinsic $\sigma_{\mathrm{sh}}$ and density of states at the Fermi level (Freimuth et al., 2010, 2015). Enhanced efficiencies can be obtained in multilayers where the FM is sandwiched between two NM with opposite spin Hall angle (Woo et al., 2014; Yu et al., 2016b). Results obtained on symmetric multilayers such as $(\mathrm{Co} / \mathrm{Pd})_{n}$ (Jamali et al., 2013), on the other hand, are more controversial because of the expected compensation of the SOT from the top and bottom interfaces and the neglected influence of thermal voltages.

Recent theoretical and experimental works pointed out the possibility to induce SOT in systems with two FM layers separated by a light NM. A spin current is generated through AHE in the first FM and injected in the second FM, leading to sizable SOT (Bose et al., 2017; Freimuth et al., 2017; Taniguchi et al., 2015) and magnetization switching (Baek et al., 2017), depending on the relative orientation of the two magnetizations. Large $\mathrm{DL}$ and FL SOT efficiencies have been reported also for FM/AF systems based on IrMn (Oh et al., 2016; Tshitoyan et al., 2015; Wu et al., 2016; Zhang et al., 2016b) and PtMn (Ou et al., 2016), which allows for in-

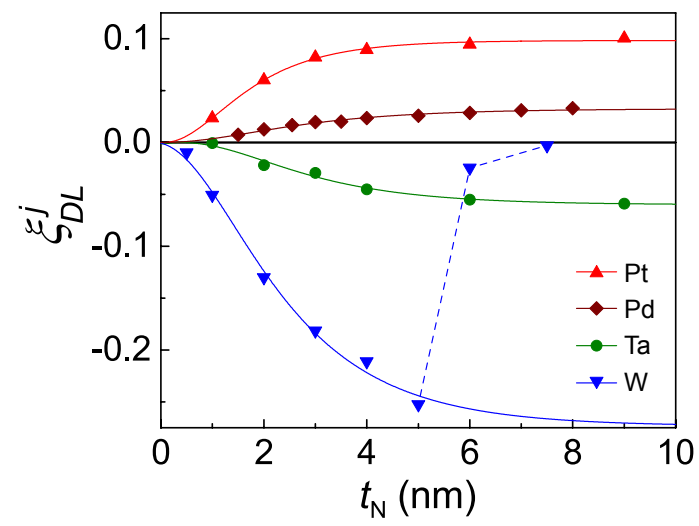

FIG. 22 (Color online) DL torque efficiency as a function of thickness in $\mathrm{NM}\left(t_{N}\right) / \mathrm{Co} / \mathrm{AlO}_{x}$ layers, where $\mathrm{NM}=\mathrm{Pt}, \mathrm{Pd}$, Ta, and W (Garello et al., 2017). The solid lines are fit to the function $\xi_{\mathrm{DL}}^{j}\left[1-\operatorname{sech}\left(t_{N} / \lambda_{\mathrm{sf}}\right)\right]$. Note that the efficiency $\xi_{\mathrm{DL}}^{j}$ of $\mathrm{W}$ drops abruptly between 5 and $6 \mathrm{~nm}$ as the crystal structure changes from the $\beta$ to the $\alpha$ phase.

cluding exchange-biased systems in SOT devices (Section IV.E.3).

In general, significant variations of the torque efficiencies have been observed depending on multilayer composition, thickness, thermal annealing protocols, interface oxidation and dusting, as well as temperature, which we briefly describe below.

a. Thickness dependence Assuming that the charge-spin conversion in multilayer systems occurs outside the FM volume, one expects the SOTs to be simply inversely proportional to the FM thickness $\left(\sim 1 / t_{F}\right)$, as the effects of the current-induced fields are inversely proportional to the magnetic volume on which they act upon, and strongly dependent on the NM thickness $\left(t_{N}\right)$ as well as on interfacial properties. The influence of $t_{F}$ on the SOT has been systematically investigated in $\mathrm{Ta} / \mathrm{CoFeB} / \mathrm{MgO}$ (Kim et al., 2013a), NiFe/Cu/Pt (Fan et al., 2013), $\mathrm{Ti} / \mathrm{CoFeB} / \mathrm{Pt}$ (Fan et al., 2014a), Co/Pt (Skinner et al., 2014), $\mathrm{Ta} / \mathrm{Pt} / \mathrm{Co} / \mathrm{MgO}$ and $\mathrm{Ta} / \mathrm{Pt} / \mathrm{Co}_{50} \mathrm{Fe}_{50} / \mathrm{MgO}$ (Pai et al., 2015), and Pd/FePd (Lee et al., 2014a), all deposited on thermally oxidized Si. Kim et al. (2013a) found that $B_{\mathrm{FL}}$ decreases strongly while $B_{\mathrm{DL}}$ remains approximately constant in $\mathrm{Ta} / \mathrm{CoFeB} / \mathrm{MgO}$ when increasing $t_{F}$ from 0.8 to $1.4 \mathrm{~nm}$. Fan et al. (2014a) showed that both fields decrease when increasing $t_{F}$ from 0.7 to $6 \mathrm{~nm}$, with $B_{\mathrm{FL}}$ dropping significantly faster than $1 / t_{F}$. The spin torque efficiencies $\xi_{\mathrm{DL}, \mathrm{FL}}^{j}$, have been found to decrease in annealed $\mathrm{Ta} / \mathrm{Pt} / \mathrm{Co}_{50} \mathrm{Fe}_{50} / \mathrm{MgO}$ layers between 0.6 and $1 \mathrm{~nm}$, but to increase in as-grown $\mathrm{Ta} / \mathrm{Pt} / \mathrm{Co} / \mathrm{MgO}$ (Pai et al., 2015), possibly because the Co thickness has to exceed the spin absorption length (i.e., the length over which the spin current is absorbed in the FM) in order to develop the full torque or because 
of strain relaxation in the $\mathrm{Pt} / \mathrm{Co}$ layer. Interestingly, the sign of the FL torque is opposite in these two systems. Skinner et al. (2014) have found a sign inversion of the FL torque in $\mathrm{Co} / \mathrm{Pt}$ for a $2 \mathrm{~nm}$ thick Co layer, which suggests that two different mechanisms compete to determine the total torque. In the case of $\mathrm{Pd} / \mathrm{FePd}$ (Lee et al., 2014a), the thickness dependence also suggests the existence of a FL torque contribution arising from the bulk of the $\mathrm{FM}$, whereas in $\mathrm{Pt} /(\mathrm{Co} / \mathrm{Tb})_{n}$ multilayers (Bang et al., 2016), both DL and FL SOTs increase with the number of repetitions, suggesting a torque contribution that originates from skew scattering due to $\mathrm{Tb}$ impurities in the Co sublayers.

The dependence of the SOT on $t_{N}$ has been the focus of many studies aimed at distinguishing the bulk and interfacial nature of the torques. In the simplest theoretical models, effects coming from the interfacial Rashba interaction should be independent of $t_{N}$, whereas effects emerging from the bulk SHE should scale as $\left[1-\operatorname{sech}\left(t_{N} / \lambda_{\mathrm{sf}}\right)\right]$ according to the profile of the spin accumulation in the NM layer (Liu et al., 2011). In addition, the Oersted field should increase linearly with $t_{N}$. Therefore, assuming that the overall structure (crystallinity, interface and inter-diffusion processes) is unchanged upon modifying $t_{N}$, analyzing the thickness-dependence of $\xi_{\mathrm{DL}}^{j}$ and $\xi_{\mathrm{FL}}^{j}$ should provide information about the physical origin of the torques. Figure 22 shows that $\xi_{\mathrm{DL}}^{j}$ of asgrown $\mathrm{Co} / \mathrm{AlO}_{x}$ layers deposited on $\beta$ - Ta, $\beta$-W, and $\mathrm{Pt}$ increases monotonically with $t_{N}$ up to saturation, which agrees well with the SHE model assuming a spin diffusion length of the order of $1.5 \mathrm{~nm}$ for all metals. Such a trend is common to a variety of systems based on Ta (Torrejon et al., 2014), W (Hao and Xiao, 2015), Pt (Nguyen et al., 2016), and Pd (Ghosh et al., 2017), suggesting that the SHE is the dominant source of the spin current causing the DL torque. Recent theoretical work, however, has pointed out that a similar $t_{N}$ dependence is expected for a purely Rashba-like DL torque due to interface spindependent scattering (Amin and Stiles, 2016b; Haney
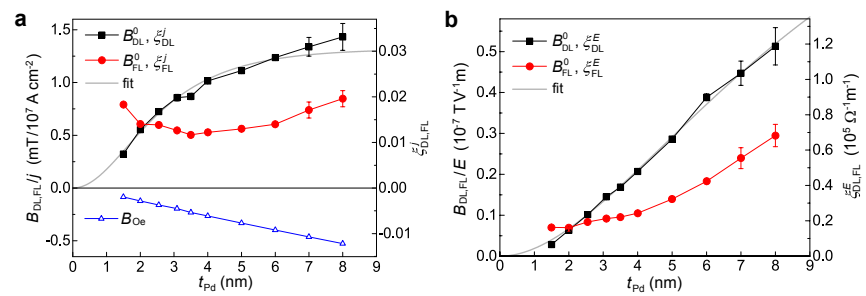

FIG. 23 (Color online) SOT efficiency in $\mathrm{Pd}(\mathrm{t}) / \mathrm{Co}(0.6) / \mathrm{AlO}_{x}$ trilayers as a function of $\mathrm{Pd}$ thickness. (a) $\xi_{\mathrm{DL}, \mathrm{FL}}^{j}$ and (b) $\xi_{\mathrm{DL}, \mathrm{FL}}^{E}$ differ significantly from each other due to the strong decrease of the Pd resistivity with increasing thickness (Ghosh et al., 2017). The FL efficiency is shown after subtraction of the Oersted field contribution $B^{O e}=\mu_{0} j_{\mathrm{Pd}} t_{\mathrm{Pd}} / 2$ shown by the open triangles in (a). et al., 2013b), so that separating the bulk and interface contributions to $\xi_{\mathrm{DL}}^{j}$ is not straightforward. Moreover, a change of sign of both $\xi_{\mathrm{DL}}^{j}$ and $\xi_{\mathrm{FL}}^{j}$ has been reported for $\mathrm{Ta} / \mathrm{CoFeB} / \mathrm{MgO}$ (Allen et al., 2015; Kim et al., 2013a) and $\mathrm{Hf} / \mathrm{CoFeB} / \mathrm{MgO}$ (Akyol et al., 2016; Ramaswamy et al., 2016) at $t_{\mathrm{Ta}} \approx 0.5 \mathrm{~nm}$ and $t_{\mathrm{Hf}} \approx 2 \mathrm{~nm}$, respectively, indicating that there are different mechanisms contributing to the torques that may compete or reinforce each other.

Further, calculations based on the drift-diffusion model of the SHE predict that the DL and FL torques should have a similar dependence on $t_{N}$ and be proportional to the real and imaginary part of the spin mixing conductance of the FM/NM interface, respectively, which naturally leads to $\xi_{\mathrm{DL}}^{j} \gg \xi_{\mathrm{FL}}^{j}$ (Haney et al., 2013b).

Several reports, however, show that $\xi_{\mathrm{FL}}^{j} \gtrsim \xi_{\mathrm{DL}}^{j}$ in outof-plane as well as in-plane magnetized layers (Table II) and that the dependence of $\xi_{\mathrm{FL}}^{j}$ on $t_{N}$ differs from that of $\xi_{\mathrm{DL}}^{j}$ in systems based on Ta (Kim et al., 2013a), Pt (Fan et al., 2014a; Nguyen et al., 2016), and Pd (Ghosh et al., 2017), particulary at low thickness $\left(t_{N}<2 \mathrm{~nm}\right)$. An example of this behavior is reported in Fig. 23(a) for a perpendicularly magnetized $\mathrm{Pd} / \mathrm{Co} / \mathrm{AlO}_{x}$ layer, where $\xi_{\mathrm{FL}}^{j}$ clearly departs from the monotonic increase of $\xi_{\mathrm{DL}}^{j}$ as a function of $t_{\mathrm{Pd}}$. Remarkably, the thickness dependence changes when the SOT efficiency is normalized to the electric field, as in Fig. 23(b), showing that $\xi_{\mathrm{DL}}^{E}$ and $\xi_{\mathrm{FL}}^{E}$ do not saturate up to $t_{N}=8 \mathrm{~nm}$ and that $\xi_{\mathrm{FL}}^{E}$ extrapolates to a finite value at $t_{\mathrm{Pd}}=0$. The difference between $\xi_{\mathrm{DL}, \mathrm{FL}}^{E}$ and $\xi_{\mathrm{DL}, \mathrm{FL}}^{j}$ also suggests that the thickness dependence should be analyzed with care in films when the resistivity is not homogeneous (Ghosh et al., 2017; Nguyen et al., 2016). Moreover, in such a regime, spin transport is not diffusive and scattering effects such as spin swapping can produce additional torques (Saidaoui and Manchon, 2016).

b. Interfacial tuning The transport of charge and spin in multilayer systems is strongly affected by interface scattering and discontinuities in the electronic band structure, as is well known from early studies of the giant magnetoresistance (Levy, 1994; Parkin, 1993). Thus, significant variations of the SOTs are expected upon modification of the interfaces, even when the spin accumulation originates in the bulk of the NM layer. Experimentally, it has been shown that the DL and FL SOTs change dramatically upon annealing and consequent intermixing of $\mathrm{Pt} / \mathrm{Co} / \mathrm{AlO}_{x}$ (Garello et al., 2013) and $\mathrm{Ta} / \mathrm{CoFeB} / \mathrm{MgO}$ (Avci et al., 2014b), as well as upon the insertion of different spacer layers between the FM and the NM that is considered to be the main source of spin accumulation (Fan et al., 2013; Pai et al., 2014; Zhang et al., 2015c). The insertion of a light metal such as $\mathrm{Cu}$ has been pursued with the intention of removing the interfacial spin- 

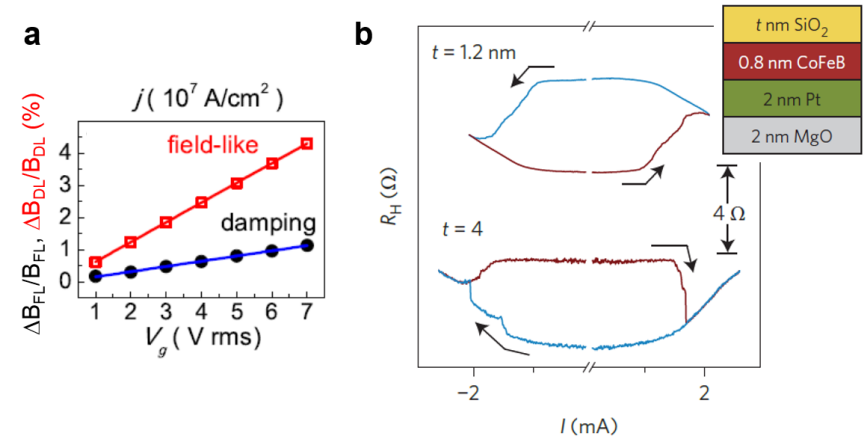

FIG. 24 (Color online) (a) Effect of gate voltage on the FL and DL SOT in Pt/Co/ $\mathrm{Al}_{2} \mathrm{O}_{3}$ (Liu et al., 2014a). (b) Inversion of the polarity of current-induced switching for different thickness of the oxide capping layer in $\mathrm{Pt} / \mathrm{CoFeB} / \mathrm{SiO}_{2}$ (Qiu et al., 2015).

orbit coupling. Fan et al. (2014a, 2013) measured a FL torque that decreases smoothly with the thickness of the $\mathrm{Cu}$ spacer in $\mathrm{Pt} / \mathrm{Cu} / \mathrm{NiFe}$, indicating a nonlocal origin, but also that the $\xi_{\mathrm{FL}}^{j} / \xi_{\mathrm{DL}}^{j}$ ratio of $\mathrm{CoFeB} / \mathrm{Cu} / \mathrm{Pt}$ has a discontinuity around $t_{C u}=0.7 \mathrm{~nm}$, which points towards a modified interface effect. In fact, the insertion of a light metal, while reducing the magnetic proximity effect between the NM and the FM, does not completely eliminate the interfacial spin-orbit coupling. Rather, it creates two additional interfaces on either sides of the light metal layer, with different spin accumulation and scattering properties. The latter effect is evident when considering that the SOTs change by as much as $50 \%$ for a $\mathrm{Cu}$ spacer thickness of the order of $1 \mathrm{~nm}$ (Fan et al., 2014a; Nan et al., 2015), which is two orders of magnitude smaller than the spin diffusion length in $\mathrm{Cu}$.

The insertion of a spacer layer can also modify the ability of the FM to absorb the incoming spin current, or the so-called transparency of the FM interface (Nguyen et al., 2015; Zhang et al., 2015c). A typical case is that of Hf, which has been shown to improve the SOT efficiency in $\mathrm{W} / \mathrm{Hf} / \mathrm{CoFeB} / \mathrm{MgO}$ and $\mathrm{Pt} / \mathrm{Hf} / \mathrm{CoFeB} / \mathrm{MgO}$ whilst promoting perpendicular magnetic anisotropy (Pai et al., 2014) and reducing the magnetic damping (Nguyen et al., 2015). Changes in the SOT efficiency in such cases are usually interpreted in terms of an enhanced spin mixing conductance, which may also explain why the DL torque efficiency changes for different FM coupled to the same NM, as observed, e.g., in $\mathrm{Pt} / \mathrm{Co} / \mathrm{TaN}\left(\xi_{\mathrm{DL}}^{j}=0.11\right)$ and $\mathrm{Pt} / \mathrm{NiFe} / \mathrm{TaN}\left(\xi_{\mathrm{DL}}^{j}=0.05\right)$ (Zhang et al., 2015c). Such a phenomenological parameter, however, accounts for the transmission of the bulk spin current as much as for the generation of interface spin currents, so that its use to estimate an asymptotic value of the bulk SHE in NMs can be questioned. Moreover, the spacer layer itself can be regarded as a source of spin current, as has been shown in the case of Hf (Akyol et al., 2016; Ramaswamy et al., 2016).
Another interesting aspect is the control of magnetic properties through interfacial oxidation (Manchon et al., 2008; Monso et al., 2002; Rodmacq et al., 2009) or gate voltage (Bauer et al., 2015; Maruyama et al., 2009; Shiota et al., 2012; Wang et al., 2012a; Weisheit et al., 2007), which is connected to the dependence of the interfacial magnetic anisotropy on the electron density tuning of ultrathin films. This control has proven efficient in the current-driven DW motion as well as interfacial magnetism and has been recently extended to SOTs (Emori et al., 2014; Liu et al., 2014a; Qiu et al., 2015). Miron et al. (2011a) first showed that moderate oxidation of $\mathrm{Pt} / \mathrm{Co} / \mathrm{AlO}_{x}$ favors current-induced switching, as recently confirmed in $\mathrm{Pt} / \mathrm{Co} / \mathrm{CoO}_{x}$ layers oxidized in air, in which up to a two-fold enhancement of the SOT efficiency was measured relative to $\mathrm{Pt} / \mathrm{Co} / \mathrm{MgO}$ (Hibino et al., 2017). On the other hand, Liu et al. (2014a) demonstrated that both FL and DL torques can be modified by gating $\mathrm{Pt} / \mathrm{Co} / \mathrm{Al}_{2} \mathrm{O}_{3}$ multilayers [Fig. 24(a)], obtaining an enhancement of $4 \%$ (1\%) of the FL (DL) torque for a gate voltage of about $7 \mathrm{~V}$. Since the gate voltage essentially modifies the electrical dipole of the $\mathrm{Co} / \mathrm{Al}_{2} \mathrm{O}_{3}$ interface and leaves the SHE from Pt unaffected, this observation provides some indication about the origin of the SOTs in this system. Liu et al. (2014a) estimate that the SHE does not contribute to more than $20 \%$ of the FL torque, while the interface spin-orbit coupling produces about 50\% of DL torque. Emori et al. (2014) carried out measurements on gated $\mathrm{Pt} / \mathrm{Co} / \mathrm{GdO}_{x}$, showing that the $\mathrm{Co} /$ interfacial oxidation state can be reversibly controlled with a gate voltage due to oxygen ion migration, which consequently affects the magnetic anisotropy (Bauer et al., 2015). This voltage-induced oxidation of Co significantly enhances $B_{\mathrm{DL}}$ whereas it weakly affects $B_{\mathrm{FL}}$ compared to the pristine state. Qiu et al. (2015) demonstrated the spectacular impact of interfacial oxidation on SOTs in $\mathrm{Pt} / \mathrm{CoFeB} / \mathrm{SiO}_{2}$, where the oxidation of the $\mathrm{CoFeB} / \mathrm{SiO}_{2}$ layer is varied continuously. They reported that the sign of both DL and FL torques changes from positive to negative when increasing the oxidation of $\mathrm{CoFeB}$ [see Fig. 24(b)]. The authors attributed this change of sign to the increase of the orbital moment of $\mathrm{Fe}$ and Co upon oxidation (Nistor et al., 2011; Yang et al., 2011). This results in an enhancement of the interfacial SOT at the upper $\mathrm{CoFeB} / \mathrm{SiO}_{2}$ interface that can even dominate over the SOT arising from the bottom $\mathrm{Pt} / \mathrm{CoFeB}$.

Other reports reveal an enhancement of $\xi_{\mathrm{DL}}^{j}$ from -0.14 to -0.49 upon oxidation of $\mathrm{W}$ in $\mathrm{W} / \mathrm{CoFeB} / \mathrm{TaN}$ (Demasius et al., 2016) and the emergence of strong SOT in asgrown $\mathrm{SiO}_{x} / \mathrm{Co} / \mathrm{Cu}$ (Verhagen et al., 2015) and oxidized $\mathrm{SiO}_{x} / \mathrm{NiFe} / \mathrm{Cu}$ layers (An et al., 2016), with contrasting evidence on the role played by the oxidized interfaces. These experiments show that interfacial spin-orbit coupling can produce significant FL and DL torques, but also that a detailed microstructural analysis of the bulk 
vs interface oxidation is required to understand the role of oxygen in inducing or modifying the SOT.

Finally, Qiu et al. (2016) recently demonstrated a 3-fold enhancement of the SOT magnitude in a $\mathrm{Pt} / \mathrm{Co} / \mathrm{Ni} / \mathrm{Co}$ multilayer by capping the system with $\mathrm{Ru}$. This result is interpreted in terms of enhanced spin absorption induced by the negative spin polarization arising at the $\mathrm{Co} / \mathrm{Ru}$ interface (Nozaki et al., 2004) and could partly explain the very large SOT magnitude measured in synthetic AF DWs (Yang et al., 2015). Recent work on $\mathrm{IrMn}_{3} / \mathrm{NiFe}$ epitaxial layers also shows that $\xi_{\mathrm{DL}}^{j}$ has a facet-dependent contribution, which arises from the different orientation of the Mn magnetic moments at different interfaces (Zhang et al., 2016b).

c. Angular dependence As mentioned in Section IV.A, the SOTs are anisotropic, i.e., their magnitude changes depending on the magnetization direction in a way that is more complex than described by Eq. (1). The magnitude of this anisotropy is characterized by the coefficients $\tau_{\mathrm{DL}, \mathrm{FL}}^{\{2 n\}}$ in Eq. (45) and can be quite large, as measured in $\mathrm{Pt} / \mathrm{Co} / \mathrm{AlO}_{x}$ (Garello et al., 2013), Ta/CoFeB/MgO (Avci et al., 2014b; Qiu et al., 2014), and Pd/Co/AlO (Ghosh et al., 2017). Figure 25 shows that both FL and DL torques increase in absolute value when the magnetization points in-plane, which is the typical behavior observed in metal multilayers. The anisotropies of the FL and DL components differ from each other and can reach up to a factor of 4 depending on the material and annealing conditions.

The angular dependence of the SOT, although quite

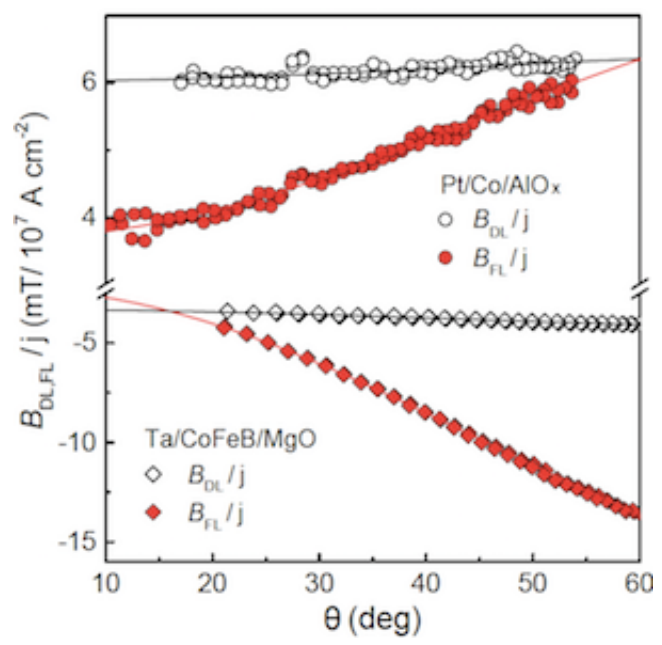

FIG. 25 (Color online) Angular dependence of $B_{\mathrm{DL}}$ and $B_{\mathrm{FL}}$ measured in as-grown $\mathrm{Pt}(3) / \mathrm{Co}(0.6) / \mathrm{AlO}_{x}$ (Garello et al, 2013) and $\mathrm{Ta}(3) / \mathrm{CoFeB}(0.9) / \mathrm{MgO}$ (Avci et al., 2014b) at room temperature. The angle $\theta$ between the magnetization and the $z$-axis is determined by anomalous Hall resistance measurements. The solid lines are fits to the function $B_{\theta}^{\mathrm{DL}, \mathrm{FL}}=B_{0}^{\mathrm{DL}, \mathrm{FL}}+B_{2}^{\mathrm{DL}, \mathrm{FL}} \sin ^{2} \theta$.
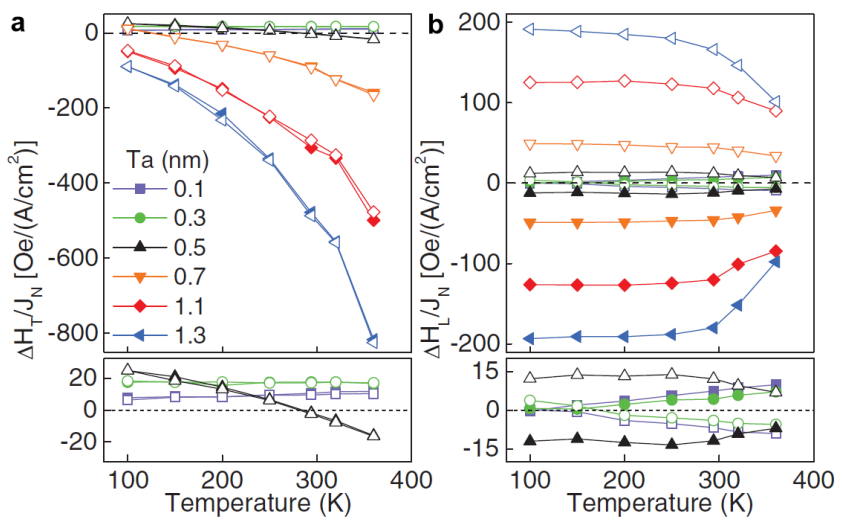

FIG. 26 (Color online) Temperature dependence of (a) $B_{\mathrm{FL}} / j$ $\left(\Delta H_{\mathrm{T}} / j\right)$ and (b) $B_{\mathrm{DL}} / j\left(\Delta H_{\mathrm{L}} / j\right)$ in $\mathrm{Ta} / \mathrm{CoFeB}(1) / \mathrm{MgO}(2)$ layers with different Ta thickness (Kim et al., 2014). The bottom panels show a magnified view of the fields for the thinner Ta layers. Solid and open symbols correspond to the magnetization pointing along $+\mathbf{z}$ and $-\mathbf{z}$, respectively.

general, provides additional clues about the physics taking place in these ultrathin multilayers. Three possible physical mechanisms can generate such an angular dependence: (i) the presence of D'yakonov-Perel relaxation (Ortiz Pauyac et al., 2013), (ii) the distortion of the Fermi surface when changing the magnetization direction due to strong spin-orbit coupling (Haney et al., 2013a; Lee et al., 2015), and (iii) the angular dependence of the interfacial mixing conductance, i.e., the change of spin absorption as a function of the magnetization direction Amin and Stiles (2016a). All these effects arise at the interface, but additional effects related to spin scattering in the NM may also be relevant, such as, e.g., spin swapping (Saidaoui and Manchon, 2016). Interestingly, Qiu et al. (2014) reported that the angular dependence of the two torque components vanishes when decreasing the temperature, an observation that highlights the importance of scattering events in the emergence of the angular dependence of the SOTs.

A strong anisotropy of the DL SOT has been reported also in magnetic topological insulators, namely in $\left(\mathrm{Cr}_{0.08} \mathrm{Bi}_{0.54} \mathrm{Sb}_{0.38}\right)_{2} \mathrm{Te}_{3} /\left(\mathrm{Bi}_{0.5} \mathrm{Sb}_{0.5}\right)_{2} \mathrm{Te}_{3}$ bilayers, where $\xi_{\mathrm{DL}}^{j}$ ranges from from 140 to 425 as the magnetization rotates from in-plane to out-of-plane (Fan et al., 2014b). The anisotropy of this system is opposite to that of $\mathrm{NM} / \mathrm{FM}$ bilayers and is qualitatively consistent with theoretical predictions (Ndiaye et al., 2017) related to the spin-momentum locking of the topological surface states (see Subsection III.G).

d. Temperature dependence A way to obtain information on the physics governing the SOTs is to measure their magnitude as a function of temperature. In $\mathrm{Ta} / \mathrm{CoFeB} / \mathrm{MgO}$, Qiu et al. (2014) reported that the FL torque decreases linearly when reducing the sample tem- 
perature, while the DL torque remains mostly unaffected. A qualitatively similar behavior was observed by Kim et al. (2014) in similar structures, i.e., the FL torque decreases dramatically with the temperature, while the DL torque increases from 400 to $300 \mathrm{~K}$ and saturates at lower temperatures (Fig. 26). Since the Ta resistivity is almost constant between 100 and $400 \mathrm{~K}$, the relative independence of the DL torque on temperature is consistent with the DL torque being driven by the intrinsic SHE of Ta. In contrast, the strong decrease of the FL torque suggests that scattering events involving phonons and magnons (usually stronger at disordered interfaces) play an important role in the emergence of this component. Studies of the temperature dependence of the SOTs in Pt-based structures, on the other hand, show that the FL and DL SOTs are both approximately constant with temperature in as-grown $\mathrm{Pt} / \mathrm{Co} / \mathrm{MgO}$, whereas both increase with temperature in annealed $\mathrm{Pt} / \mathrm{CoFeB} / \mathrm{MgO}$ (Pai et al., 2015). In the latter case, the FL torque shows a much stronger change compared to the DL torque and even changes sign, from parallel to antiparallel to the Oersted field, at around $125 \mathrm{~K}$. Interestingly, in $\mathrm{NM}$ where extrinsic effects dominate, such as $\mathrm{CuAu}$ alloys, the DL torque decreases upon reducing the temperature, consistently with an extrinsic bulk-like SHE, while the FL torque increases Wen et al. (2017). Such a behavior supports the view that intrinsic as well as extrinsic mechanisms contribute in different proportion to the FL and DL torques.

\section{FM/semiconductor layers}

We now turn from purely metallic systems to FM/semiconductor bilayers, in which the semiconductor has a specific crystal structure that brings about additional symmetries on top of the one arising from the interface. For instance, in zinc-blende lattices under strain, such as GaAs, SOTs can be generated via iSGE driven by Rashba and Dresselhaus spin-orbit coupling, as well as by bulk SHE (see Section V). Differently from the commonly studied polycrystalline transitionmetal FM/NM samples, where the iSGE-based and the SHE-based mechanisms are indistinguishable in the lowest order torque terms (Garello et al., 2013), the dependence of the torques on the angle of the current relative to the high symmetry directions of the semiconductor crystal provides a direct means to disentangle the SHE and iSGE contributions. To prove this concept, Skinner et al. (2015) investigated the SOTs of a single crystal $\mathrm{Fe}(2 \mathrm{~nm}) /(\mathrm{Ga}, \mathrm{Mn}) \mathrm{As}(20 \mathrm{~nm})$ bilayer using the STFMR technique. The GaAs host was doped with high enough concentration of substitutional $\mathrm{Mn}_{\mathrm{Ga}}$ acceptors to increase the semiconductor conductivity, whilst low enough so that the semiconductor remains paramagnetic at room temperature. From the measurements it was
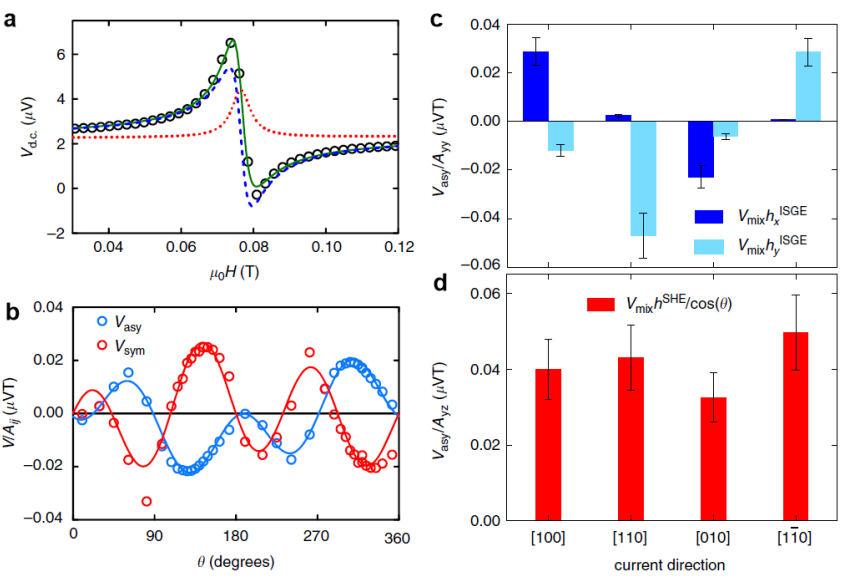

FIG. 27 (Color online) (a) Electrical excitation and detection of FMR induced by a $16.245 \mathrm{GHz}$ RF current in a $\mathrm{Fe}(2$ $\mathrm{nm}) /(\mathrm{Ga}, \mathrm{Mn}) \operatorname{As}(20 \mathrm{~nm})$ bilayer. A typical ST-FMR curve (points) is shown as a function of external field. The dc voltage is fitted (solid green line) by a combination of symmetric (red dotted line) and antisymmetric (blue dashed line) Lorentzians. (b) Dependence of the fitted Lorentzian amplitudes on the in-plane magnetization angle for a device with current in the [010] direction. (c) iSGE dependence on the direction of the current. The fitted in-plane field coefficients (representing the FL torque) for a set of devices in different crystal directions. (d) The fitted out-of-plane field coefficient (representing the DL torque) for the same devices. Adapted from Skinner et al. (2015).

concluded that the FL and DL torques have similar magnitudes. By measuring devices where the current is applied along different crystal directions of the semiconductor, it was then shown that the iSGE with a characteristic Dresselhaus symmetry induces only the FL torque in the adjacent Fe, whereas the SHE spin-current, generated inside the paramagnetic $p$-doped GaAs layer, is absorbed in the weakly spin-orbit coupled $\mathrm{Fe}$ in the form of the DL torque. Therefore in this bilayer, the iSGE and SHE mechanisms could be separated into the distinct SOT components. Hupfauer et al. (2015) further explored the influence of the Dresselhaus and Rashba inversion asymmetry in epitaxial Fe/GaAs(001) films, showing the emergence of a crystalline magnetoresistance related to the interfacial spin-orbit fields.

Evidence of strong SOTs due to the iSGE has been observed also in heterostructures involving transition metal dichalcogenides and metallic FM. Van der Waals crystals provide a unique platform for generating SOTs because they have strong spin-orbit coupling, a range of broken crystal symmetries, and can be prepared as monolayer crystalline films by exfoliation or chemical vapor deposition methods (Manchon et al., 2015; Xu et al., 2014). Shao et al. (2016) showed that the FL torque in $1 \mathrm{~nm}$ CoFeB deposited on monolayer $\mathrm{MoS}_{2}$ and $\mathrm{WSe}_{2}$ is of the order of $0.1-0.14 \mathrm{mT} / 10^{7} \mathrm{Acm}^{-2}$, independently of temperature, and is consistent with iSGE-induced spin 
accumulation, whereas the DL torque is negligibly small. Sizable DL SOTs, on the other hand, have been reported for NiFe deposited on $\mathrm{MoS}_{2}$ (Zhang et al., 2016c) and on the semi-metal $\mathrm{WTe}_{2}$ (MacNeill et al., 2017). The latter case is of particular interest as the surface crystal structure of $\mathrm{WTe}_{2}$ has only one mirror plane and no two-fold rotational invariance about the $c$-axis, which allows for a DL torque that is directed out-of-plane when the current is applied along a low-symmetry axis of the surface. Such a DL torque is forbidden by symmetry in NM/FM layers, where the direction of the incoming spin polarization is in-plane. The possibility of controlling the allowed symmetry of the DL SOT in multilayer samples is particularly attractive for counteracting the torque due to magnetic damping during magnetization reversal in systems with perpendicular magnetic anisotropy. Further, the current-induced spin polarization in two-dimensional (2D) materials is expected to be extremely sensitive to gating, thus allowing for tuning the SOT efficiency.

\section{Surface alloys and two-dimensional gases}

Spin pumping measurements performed on heterostructures consisting of a FM layer and an interface alloy with strong Rashba coupling, such as $\mathrm{Ag} / \mathrm{Bi}$ (Rojas-Sánchez et al., 2013), Cu/Bi (Isasa et al., 2016), and $\mathrm{Cu} / \mathrm{Bi}_{2} \mathrm{O}_{3}$ (Karube et al., 2016), have revealed large charge-spin conversion efficiencies due to the iSGE. Owing to the interfacial nature of the charge current $\mathfrak{j}_{c}$ involved in this process, the charge-spin conversion is given by the iSGE (or Rashba-Edelstein) "length" $\lambda_{\mathrm{REE}}=\mathfrak{j}_{c} / j_{s}$, where $\mathfrak{j}_{c}$ is measured in $\mathrm{Am}^{-1}$ and $j_{s}$ in $\mathrm{Am}^{-2}$. In the framework of the Rashba model, it can be shown that $\lambda_{\mathrm{REE}}=\alpha_{\mathrm{R}} \tau_{s} / \hbar$, where $\alpha_{\mathrm{R}}$ is the Rashba coupling strength and $\tau_{s}$ the relaxation time of the spin/momentum polarization at the Rashba-split Fermi surface (Gambardella and Miron, 2011; RojasSánchez et al., 2013). Typical values of $\lambda_{\text {REE }}$ range from 0.1-0.3 nm in NiFe/Ag/Bi layers (Rojas-Sánchez et al., 2013; Zhang et al., 2015b) to $-0.6 \mathrm{~nm}$ in $\mathrm{NiFe} / \mathrm{Cu} / \mathrm{Bi}_{2} \mathrm{O}_{3}$ (Karube et al., 2016). Because of the much slower spin relaxation in insulating systems, $\lambda_{\mathrm{REE}}$ can reach extremely large values in heterostructures including a $2 \mathrm{D}$ electron gas confined at a polar oxide interface such as $\mathrm{NiFe} / \mathrm{LaAlO}_{3} / \mathrm{SrTiO}_{3}$, in which $\lambda_{\mathrm{REE}}$ can be tuned between -6 and $2 \mathrm{~nm}$ by electric gating (Lesne et al., 2016; Song et al., 2017). Due the reciprocity between spinorbit charge pumping and SOT, one can expect to generate sizable DL and FL SOT by charge injection in such systems, as was recently demonstrated by Jungfleisch et al. (2016) in the case of $\mathrm{NiFe} / \mathrm{Ag} / \mathrm{Bi}$. For comparison with SOT in NM/FM layers, one can convert the effective spin Hall angle into $\lambda_{\mathrm{REE}}$ by taking $\lambda_{\mathrm{REE}}=2 \theta_{\mathrm{sh}} t_{I}$, where $t_{I} \approx 2 \lambda_{\text {sf }}$ is the "thickness" of the interface layer in which the charge-spin conversion takes place (Rojas-
Sánchez et al., 2013). For values of $\theta_{\text {sh }}$ between 0.1 and 0.3 , and $\lambda_{\mathrm{sf}}=1.5-2 \mathrm{~nm}$ as typical of $\mathrm{Pt}$, Ta, and $\mathrm{W}$, one obtains $\lambda_{\mathrm{REE}}=0.15-0.6 \mathrm{~nm}$, which is comparable to $\lambda_{\mathrm{REE}}$ of the $\mathrm{Ag} / \mathrm{Bi}$ interface.

\section{4. ferromagnet/topological insulator layers}

$3 \mathrm{D}$ topological insulators are materials that have an insulating bulk and spin-momentum-locked metallic surface states (Hasan and Moore, 2011). Since they involve heavy elements ( $\mathrm{Sn}, \mathrm{Sb}, \mathrm{Te}, \mathrm{Bi}$, etc.), they exhibit strong spin-orbit coupling and are expected to show large charge-spin conversion efficiency. In these materials, the topological surface state is immune to scattering from nonmagnetic impurities due to the time reversal symmetry protection. Recent reports have confirmed that such a surface state is intact in $\mathrm{Bi}_{2} \mathrm{Se}_{3}$ covered with Fe (Honolka et al., 2012; Scholz et al., 2012) or Co (Ye et al., 2012) with in-plane magnetic anisotropy. Spin-charge coupling effects have been reported by spin pumping (Deorani et al., 2014; Jamali et al., 2015; Kondou et al., 2015; Rojas-Sánchez et al., 2016; Shiomi et al., 2014) and magnetoresistance measurements (Ando, 2014; Li et al., 2014; Yasuda et al., 2016).

Direct current-induced SOT on the FM layer has been demonstrated by ST-FMR measurement (Mellnik et al., 2014; Wang et al., 2015), gate control of the torque efficiency (Fan et al., 2016), and lowtemperature magnetization switching (Fan et al., 2014b). In these experiments, the reported DL torque efficiency is widely distributed from 0.01 in spin pumping measurements to 2 using ST-FMR measurements in $\mathrm{Bi}_{2} \mathrm{Se}_{3} / \mathrm{NiFe}$ or $\mathrm{Bi}_{2} \mathrm{Se}_{3} / \mathrm{CoFeB}$. It reaches even larger values in $\left(\mathrm{Bi}_{0.5} \mathrm{Sb}_{0.5}\right)_{2} \mathrm{Te}_{3} /\left(\mathrm{Cr}_{0.08} \mathrm{Bi}_{0.54} \mathrm{Sb}_{0.38}\right)_{2} \mathrm{Te}_{3}$ heterostructures (Fan et al., 2014b), where, however, the data analysis is complicated by nonlinear Hall effects (Yasuda et al., 2017). We emphasize that the theoretical understanding of these results (large DL torque, angular dependence, gate control) is still in its infancy. As a matter of fact, in all these samples bulk transport coexists with surface transport and it is unclear how they both contribute to the overall charge-spin conversion process (see Section III.G).

Progress in the growth of topological insulators and in the deposition of homogeneous magnetic layers, free from dead layers, has finally led to the demonstration of room temperature SOT-driven switching in these systems. Yasuda et al. (2017) observed current-driven switching in $\mathrm{Cr}_{x}\left(\mathrm{Bi}_{1-y} \mathrm{Sb}_{y}\right)_{2-x} \mathrm{Te}_{3} /\left(\mathrm{Bi}_{1-y} \mathrm{Sb}_{y}\right)_{2} \mathrm{Te}_{3}$ with critical currents of $\sim 6 \times 10^{6} \mathrm{~A} / \mathrm{cm}^{2}$, while Wang et al. (2017) lowered the critical current down to $\sim 6 \times 10^{5}$ $\mathrm{A} / \mathrm{cm}^{2}$ in $\mathrm{Bi}_{2} \mathrm{Se}_{3} / \mathrm{NiFe}$. Both experiments concern inplane magnetized samples. Alternatively, Han et al. (2017) demonstrated SOT switching in a perpendicularly magnetized $\mathrm{Bi}_{2} \mathrm{Se}_{3} / \mathrm{CoTb}$ bilayer at a critical cur- 
rent of $\sim 3 \times 10^{6} \mathrm{~A} / \mathrm{cm}^{2}$. All the above samples were realized by molecular beam epitaxy, a technique difficult to transfer to industrial processing. This obstacle has been seemingly overcome by Mahendra et al. (2017) who used sputtering to grow perpendicularly magnetized $\mathrm{Bi}_{2} \mathrm{Se}_{3} / \mathrm{Ta} / \mathrm{CoFeB} / \mathrm{Gd} / \mathrm{CoFeB}$ (Ta here is only 0.5 $\mathrm{nm}$ thick to induce perpendicular magnetic anisotropy). Due to its polycrystalline nature, the $\mathrm{Bi}_{2} \mathrm{Se}_{3}$ substrate is highly resistive, one order of magnitude larger than (Mellnik et al., 2014), thereby enabling current to flow mostly through the interface and in the FM layer, which enhances the SOT efficiency. However, the role, if any, of the topological surface states in the sputtered layers remains to be proven, together with the stoichiometric profile of the $\mathrm{Bi}_{2} \mathrm{Se}_{3}$ films.

Notice that in all the studies of topological insulators the current densities and SOT efficiencies must be taken with care, as the current distribution in such heterostructures is determined by the balance between the surface and the bulk, defect-mediated conduction, and is therefore highly inhomogeneous. These achievements nonetheless constitute a crucial milestone towards the implementation of topological insulators in technology-relevant devices.

\section{Established features of SOTs and open questions}

The complexity and interplay of the different chargespin conversion mechanisms outlined in Section III underpins an ongoing debate on the origin of SOTs and on strategies to improve their efficiency. Below, we summarize the most important findings drawn from experimental investigations of metallic layers:

- In most NM/FM systems the sign of the DL torque is consistent with that of the SHE of the bulk NM. Additionally, NM elements with strong SHE present large DL torques. The magnitude and the sign of the DL torque can be modified by changing the oxidation state or the capping layer of the FM interface that is not in contact with the NM. Significant DL torques have been reported also for FM layers adjacent to metal alloys and oxide layers with a strong localized spin-orbit potential.

- The FL torque is of the same order of magnitude as the DL torque. The sign and magnitude of the FL torque are not consistent with the predictions of the drift-diffusion model based on the bulk SHE.

- The DL and FL torques typically increase with the thickness of the NM layer and saturate after a few $\mathrm{nm}$. The dependence of the two torques on the NM thickness is not the same.

- The temperature dependence of the FL and DL torques is different, indicating the distinct role of (phonon and magnon) scattering.
- Extrinsic effects related to both interface and bulk scattering are significant and can give rise to both DL and FL torques. The SOTs are typically large in high resistivity metals and correlate with the presence of strong SMR in FM/NM layers (Nakayama et al., 2013) and crystalline AMR in FM/semiconductor layers.

- The angular dependence of the torques show that interfacial spin-orbit coupling, either through D'yakonovPerel relaxation, Fermi surface distortion or anisotropic mixing conductance, plays a relevant role.

- The insertion of a nonmagnetic light metal spacer between the FM and a NM layer reduces magnetic proximity effects in the NM, but creates additional interfaces that can contribute to the generation of spin currents. Both the DL and FL torques change upon the insertion of nonmagnetic and magnetic spacers.

- 2D materials and topological insulators provide large SOTs when interfaced with FM layers owing to their spin-momentum locked surface states, as well as weak bulk conductivity. The symmetry of the SOTs in these systems is determined by the crystalline structure and the current injection direction.

- Both DL and FL torques can be controlled through interface engineering, such as gate voltage, oxidation, or capping layer, which offers an efficient way to improve charge-spin conversion in $\mathrm{FM} / \mathrm{NM}$ as well as $2 \mathrm{D}$ systems.

SOT measurements in multilayer systems are often interpreted assuming either the SHE-SOT model or the Rashba-type iSGE. Such approaches are appealing because of their simplicity, but neglect important aspects of the generation of SOT. The one-dimensional driftdiffusion theory based on the bulk SHE (Section III.C) is the most commonly employed model to relate the torque amplitude to the spin Hall conductivity of the NM. Such a model includes the probability of spin transmission at the interface through the spin mixing conductance parameter, but neglects the interface-generated spin accumulation by either the iSGE or spin-dependent electron scattering. Another major limitation of this model is that it assumes constant parameters $\sigma_{N}, \lambda_{\mathrm{sf}}$, and $\theta_{\mathrm{sh}}$ throughout the NM layer, which is unjustified on both theoretical and experimental grounds. On the other hand, most SOT models based on interfacial Rashba spin-orbit coupling assume a static spin polarization localized at a sharp interface between the NM (or the oxide) and the FM. Considering the complexity of the real ultrathin magnetic multilayers involving complex orbital hybridization, disordered interfaces, spin-dependent semiclassical size effects, it is quite unclear how these two models (bulk SHE and interfacial Rashba-like iSGE) apply to real systems. Moreover, there is increasing evidence that different intrinsic effects are at play in such systems, leading, e.g., to interface-enhanced SHE (Freimuth et al., 2014b; Wang et al., 2016a), together with extrinsic effects in- 
(a)
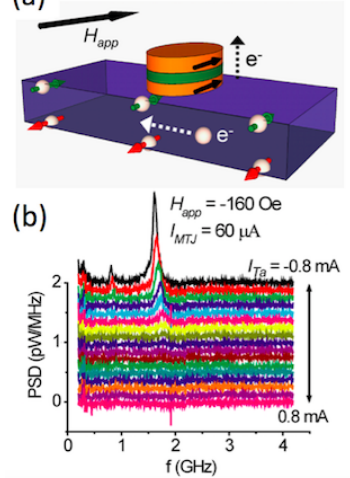

(c)

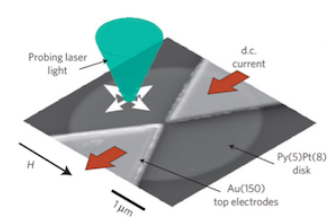

(d)

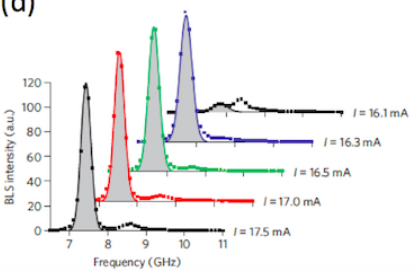

FIG. 28 (Color online) (a,c) Two configurations for the SOTinduced nano-oscillator and $(b, d)$ their corresponding excitation spectrum. (a,b) Nanopillar deposited on top of a NM (Liu et al., 2012c), and (c,d) Local injection into an extended FM (Demidov et al., 2012).

volving electron scattering from interfaces and impurities. Along this line, spin pumping experiments at $\mathrm{Bi}$ surfaces have been interpreted as evidence for either an interface-enhanced SHE (Hou et al., 2012) or the iSGE (Rojas-Sánchez et al., 2013). Angle-resolved photoemission studies, on the other hand, provide evidence that the iSGE is not a pure 2D effect in metallic thin films: the presence of magnetic exchange (Krupin et al., 2005), out-of-plane spin polarization (Takayama et al., 2011), spin-momentum locked quantum well states in the FM (Moras et al., 2015), and topologically protected surface states (Thonig et al., 2016) significantly alters the Rashba effect at metallic interfaces compared to model semiconducting heterostructures. Further, extrinsic effects such as impurity and interface scattering can induce additional spin currents that propagate through or away from the $\mathrm{NM} / \mathrm{FM}$ interface and be polarized in directions different from the standard model, calling for a generalization of the mixing conductance concept (Amin and Stiles, 2016a,b; Chen and Zhang, 2015). The interplay between all these effects thus makes it questionable to draw a clear separation between the SHE and iSGE in metallic structures, even when considering idealized theoretical models of these heterostructures.

\section{Magnetization dynamics}

SOTs offer an interesting paradigm for current-driven spin wave amplification and self-sustained magnetic oscillations. Ando et al. (2008) first observed a reduction or increase of magnetic damping according to the sign of the $\mathrm{DL}$ torque in $\mathrm{Pt} / \mathrm{NiFe}$ bilayers. Current-driven excitations in magnetic bilayers were later reported by Liu et al. (2011) (using ST-FMR - see Section IV.B.2) and Demidov et al. (2011b) (using Brillouin light scattering) in $\mathrm{NiFe} / \mathrm{Pt}$ bilayers. In these experiments, thermal

magnetic excitations were enhanced or quenched by injecting a current into the underlying Pt layer. Demidov et al. $(2011 \mathrm{a}, \mathrm{b})$ found that the current mostly excites long wavelength fluctuations while Joule heating affects short wavelength excitations. The electrical control of the magnetic damping through SOT in $\mathrm{Pt} / \mathrm{NiFe}$ bilayer has been used to enhance the spin wave propagation length in microwave guides (An et al., 2014; Demidov et al., 2014b). The electrical control of spin wave excitations has also been achieved in the magnetic insulator yttrium iron garnet (YIG) deposited on top of Pt (Wang et al., 2011).

A limiting factor for achieving self-sustained oscillations is the degeneracy of spin wave modes. If the sample is large, a significant amount of modes compete with each other to absorb the energy deposited by the SOT. In such a case, the degeneracy is high and only thermal excitations can be electrically controlled rather than current-driven coherent oscillations. Achieving selfsustained magnetic oscillations requires to lift the degeneracy by reducing the size of the sample and thereby lowering the excitation threshold and excitation bandwidth. Current-driven magnetic oscillations where reported by Liu et al. (2012c) in a 3-terminal $\mathrm{CoFeB} / \mathrm{MgO} / \mathrm{CoFeB}$ nanopillar fabricated on top of a large Ta buffer layer, and capped by an MTJ [see Fig. 28(a)]. Due to the reduced size of the nano pillar $\left(\sim 50 \times 80 \mathrm{~nm}^{2}\right)$, currentdriven oscillations were detected electrically through the MTJ [see Fig. 28(b)] and independent control of the excitation via the currents injected into the Ta layer and through the MTJ was achieved. More recently, Duan et al. (2014a,b) achieved SOT-driven spin wave damping control and self oscillation in long and narrow nanowires $(\sim 1.6 \mu \mathrm{m} \times 190 \mathrm{~nm})$, where both bulk modes and edge modes were identified.

Another successful configuration was realized by Demidov et al. (2012) by locally injecting a spin current in an extended FM layer [see Fig. 28(c)]. The local injection creates a spin wave bullet, i.e., a spin wave packet localized in space through non-linear energy losses (Slavin and Tiberkevich, 2005). This self-localization enables the selection of a small number of spin wave modes that reveal themselves in the coherent self-oscillation. Synchronization of this SOT-induced spin wave bullet with an external microwave field has been achieved by the same group (Demidov et al., 2014a). Liu et al. (2013) investigated the microwave spectrum of the nano-oscillator, observing features similar to "traditional" spin torque pointcontact oscillators, namely a spin wave "bullet" and a propagating spin wave mode (Bonetti et al., 2010; Slavin and Tiberkevich, 2005).

Finally, SOTs represent a crucial element of magnonic circuits (Chumak et al., 2015), as they provide interconversion between the spin and charge currents in an electrical conductor and the magnon currents in a magnetic insulator. In a pioneering experiment, Kajiwara et al. (2010) showed that SOTs convert a dc electric cur- 
rent flowing in a Pt wire deposited on a YIG film into a spin wave propagating through the YIG film, which can then be detected by a Pt electrode at a different location using spin-orbit charge pumping. YIG presents a specific challenge due to the low magnetic damping, which makes it harder to excite single-mode or few-modes oscillations compared, e.g., to NiFe. Xiao and Bauer (2012) argued that surface spin waves are preferentially excited compared to bulk spin waves, which renders the observation of current-driven self-oscillations very sensitive to both the size of the YIG layer and to the quality of the interface with $\mathrm{Pt}$.

Hamadeh et al. (2014) showed that the magnetic losses of spin wave modes in micron-sized YIG(20nm)/Pt(8nm) discs can be reduced or enhanced by a factor of 5 depending on the polarity and intensity of the dc current flowing through $\mathrm{Pt}$, reaching complete compensation of the damping of the fundamental mode for a current density of $3 \times 10^{7} \mathrm{~A} \mathrm{~cm}^{-2}$, and eventually inducing coherent SOT-induced self-oscillations (Collet et al., 2016), see Fig. 29. The threshold current of the self-oscillations is indeed increased by the presence of quasi-degenerate SW modes in this system. While all these measurements employed a dc current, it has also been shown that sending an ac current at $\mathrm{GHz}$ frequencies through YIG/Pt bilayers integrated into a coplanar waveguide results in FMR (Schreier et al., 2015). In this case, the magnetization
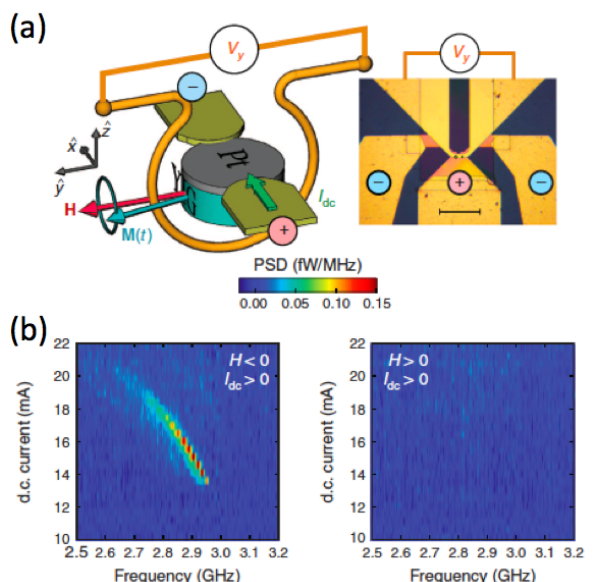

FIG. 29 (Color online) (a) Sketch of the measurement configuration and microscopy image of a device with two microdiscs connected (underneath the circles). The bias field $\mu_{0} H$ is oriented transversely to the dc current $I_{\mathrm{dc}}$ flowing in Pt. The inductive voltage $V_{y}$ produced in the antenna by the precession of the YIG magnetization $M(t)$ is amplified and monitored by a spectrum analyzer. (b) Power spectral density (PSD) maps measured on a $4 \mathrm{~mm}$ YIG/Pt disc at fixed $\left|\mu_{0} H\right|=47 \mathrm{mT}$ and variable $I_{\mathrm{dc}}$. The two panels correspond to two different polarities of $\mu_{0} H$. An auto-oscillation signal is detected above a threshold current of $\pm 13 \mathrm{~mA}$ if $\mu_{0} H \cdot I_{\mathrm{d} j} 0$, in agreement with the symmetry of the torque. Adapted from (Collet et al., 2016). dynamics is excited by the combined action of SOTs and Oersted field, with the former dominating in thin YIG samples.

Overall the SOT approach is very attractive for controlling the magnetization dynamics of a broad class of materials, including FM metals, insulators, and AF layers. Because no electric current is required to flow between the magnetic layer and the spin-orbit coupled electrodes, low-damping magnetic dielectric materials can be used as the carriers of magnetic information over large distances. Moreover, SOTs can be applied to arbitrarily large area of a magnetic film, unlike STT, which is limited to pillar-shaped nanostructures, allowing for spin wave amplification through the compensation of damping. These exciting observations thus open new perspectives for inducing the coherent emission, amplification, and detection of spin waves, ultimately leading to the efficient integration of spintronic and magnonic devices (Chumak et al., 2015).

\section{E. Magnetization switching}

The realization of current-driven magnetization switching has been a major milestone in the progress towards SOT devices. Miron et al. (2011a) and Liu et al. (2012a,b) demonstrated that, in the presence of a constant in-plane magnetic field, the magnetization direction of a perpendicularly magnetized ultrathin trilayer $\left(\mathrm{Pt} / \mathrm{Co} / \mathrm{AlO}_{x}\right.$ and $\left.\mathrm{Ta} / \mathrm{CoFeB} / \mathrm{MgO}\right)$ could be reversibly switched by injecting current densities of the order of $10^{7}-10^{8} \mathrm{~A} / \mathrm{cm}^{2}$ (see Fig. 30). This observation was soon confirmed by several groups using different magnetic stacks and NM substrates (Avci et al., 2012, 2014b; Emori et al., 2013; Pai et al., 2012; Yu et al., 2014b), as well as AFs (Fukami et al., 2016b; Oh et al., 2016; Wadley et al., 2016), magnetic insulators (Avci et al., 2017; Li et al., 2016b), and topological insulators (Han et al., 2017; Mahendra et al., 2017; Wang et al., 2017). The switching of a perpendicularly magnetized layer can be qualitatively explained by considering the combined action of the DL torque and in-plane field $B_{x}$ in a simple macrospin picture, as shown in Fig. 30(e). In the $\mathrm{Pt} / \mathrm{Co} / \mathrm{AlO}_{x}$ stack, a positive current pulse induces an effective field $\mathbf{B}_{\mathrm{DL}}$, such that the magnetization can rotate from up to down if $B_{\mathrm{DL}}$ is initially parallel to $B_{x}$, but cannot rotate from down to up if $B_{\mathrm{DL}}$ is antiparallel to $B_{x}$. When the current polarity is reversed, the sense of rotation changes, such that bipolar switching is achieved by either current or in-plane field reversal, as shown in Fig. 30(f). More generally, the transferred angular momentum is transverse to both the current direction and the normal to the plane, which alone cannot ensure reversible magnetization switching between the $+\mathbf{z}$ and $-\mathbf{z}$ directions. Hence, the DL torque must be supplemented by the in-plane field $B_{x}$ that breaks the symmetry along 

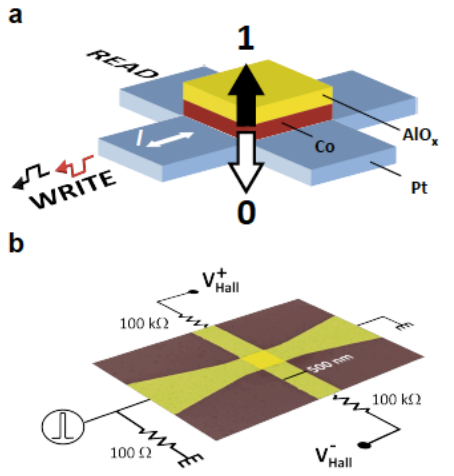

C

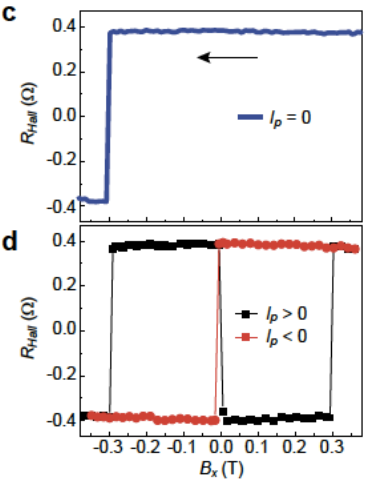

e

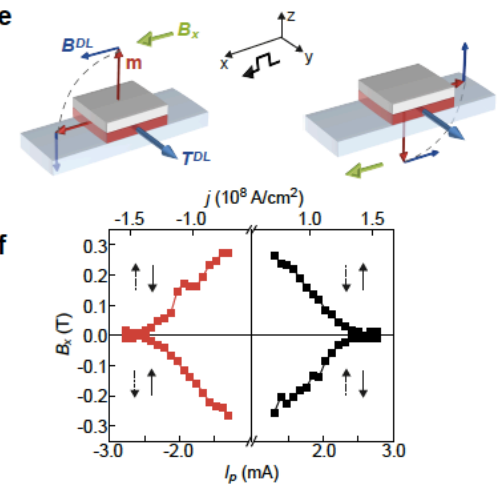

FIG. 30 (Color online) (a) Schematic of a $\mathrm{Co}(0.6 \mathrm{~nm}) / \mathrm{AlO}_{x}(2 \mathrm{~nm})$ dot patterned on top of a $3 \mathrm{~nm}$ thick Pt Hall cross. Black and white arrows indicate the equilibrium magnetization states of the Co layer. (b) Detection scheme and scanning electron micrograph of the sample. (c) $m_{z}$ measured by the anomalous Hall resistance during a downward sweep of the external field $B_{x}$ applied parallel to the current direction. The field has a $2^{\circ}$ out-of-plane tilt to unambiguously define the residual $z$ component. (d) The same measurement recorded after the injection of positive (black squares) and negative (red circles) current pulses of amplitude $I_{p}=2.58 \mathrm{~mA}$, showing bipolar switching of $m_{z}$. (e) Macrospin model showing the stable (right) and unstable (left) magnetic configurations depending on the sign of $B_{\mathrm{DL}}$ relative to $B_{x}$. (f) Switching diagram: the dots show the minimum in-plane field at which switching becomes deterministic as a function of the injected current. Dashed (solid) arrows indicate the magnetization direction before (after) switching. Adapted from Miron et al. (2011b).

the current direction and determines the outcome of the switching process. In the macrospin approximation, the threshold switching current is given by (Lee et al., 2013)

$$
j_{s w, \perp}=\frac{2 e}{\hbar} \frac{M_{s} t_{F}}{\xi_{D L}^{j}}\left(\frac{B_{k, \perp}}{2}-\frac{B_{x}}{\sqrt{2}}\right)
$$

where $B_{k, \perp}$ is the perpendicular anisotropy field. Inplane magnetized samples, on the other hand, switch at zero external field as long as the magnetization has a nonzero component in the $\mathbf{y}$ direction, which can be induced by shape anisotropy (Fukami et al., 2016a; Liu et al., 2012b). In this case, the threshold current has the same form as that of the conventional STT switching for free and fixed layers with in-plane magnetization (Sun, 2000), and is given by (Lee et al., 2013)

$$
j_{s w, \|}=\alpha \frac{2 e}{\hbar} \frac{M_{s} t_{F}}{\xi_{D L}^{j}}\left(B_{k, \|}+\frac{B_{d}}{2}\right)
$$

where $B_{k, \|}$ is the in-plane anisotropy field and $B_{d}$ the demagnetizing field. Equations 61 and 62 exemplify the relationship between the power required for switching, the thermal stability of a magnet (determined by $B_{k}$ ) and $\xi_{D L}^{j}$. However, the actual mechanism of SOT switching is more complex than coherent magnetization reversal under the action of the DL torque alone.

\section{SOT switching mechanism}

Although the macrospin model reproduces qualitatively the stability phase diagram of rather extended films (Liu et al., 2012a), magnetization switching in structures larger than the width of a DW ( $\gtrsim 10 \mathrm{~nm})$ occurs by nucleation and expansion of magnetic domains. The magnetization reversal process is thus closely related to the SOT-driven dynamics of Néel-type DW in the presence of DMI (see Section VI). Different switching models have been proposed based on micromagnetic simulations (Finocchio et al., 2013; Martinez et al., 2015; Mikuszeit et al., 2015; Perez et al., 2014) and spatiallyresolved MOKE measurements (Emori et al., 2013; Ryu et al., 2013; Safeer et al., 2016; Yu et al., 2014b). In such models, the domain nucleation is either random and thermally-assisted (Finocchio et al., 2013; Lee et al., 2014b; Perez et al., 2014) or determined by the combined action of DMI, external field, and edge effects (Martinez et al., 2015; Mikuszeit et al., 2015; Pizzini et al., 2014), followed by DW propagation across the magnetic layer driven by the DL torque. Indeed, time-resolved x-ray microscopy measurements of circular shaped $\mathrm{Pt} / \mathrm{Co} / \mathrm{AlO}_{x}$ dots show that switching is achieved within the duration of a current pulse by the fast nucleation of an inverted domain at the edge of the dot and propagation of a tilted DW across the dot. The nucleation point is deterministic and alternates between the four quadrants of the dot depending on the sign of the magnetization, $B_{x}$, DMI, DL and FL torque, as illustrated in Fig. 31.

In these samples, the switching unfolds along a reproducible and deterministic path, so that the timing and the extent of magnetization reversal can be reliably controlled by the amplitude and duration of the current pulses (Baumgartner et al., 2017). Measurements performed by time-resolved MOKE on larger dots with a thinner Co layer, on the other hand, show significant after-pulse magnetic relaxation (Decker et al., 2017), which is ascribed to long-lasting heating effects 

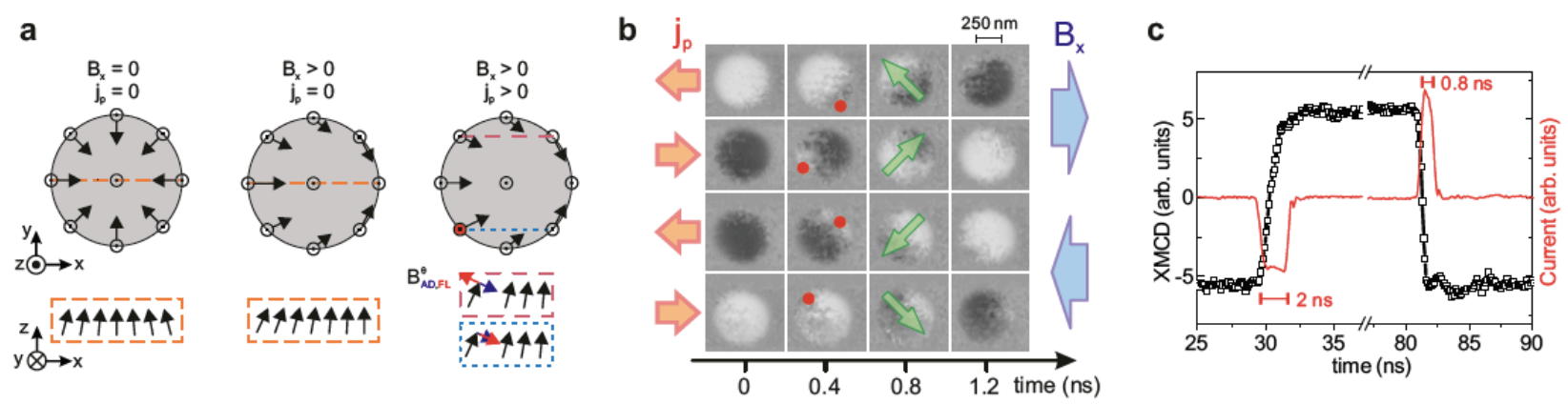

FIG. 31 (Color online) (a) Schematics of the tilting of the magnetization at the edges of a $\mathrm{Pt} / \mathrm{Co} / \mathrm{AlO} x$ dot due to the DMI (left), DMI and external field $B_{x}$ (middle), DMI, $B_{x}$, and current (right). The polar components of the DL and FL effective fields add up at the nucleation point. (b) Snapshots of the reversal process of a circular dot for different combinations of current and field measured by time-resolved scanning x-ray transmission microscopy. The red dot and green arrows indicate the nucleation point and the DW propagation direction, respectively. The pulse duration is 2 ns. (c) Time trace of the average out-of-plane magnetization (black squares) during current injection (red line). The amplitude of the first (second) pulse is $j_{p}=3.1 \times 10^{8}\left(4.4 \times 10^{8}\right) \mathrm{A} / \mathrm{cm}^{2} ; B_{x}=0.11 \mathrm{~T}$. Adapted from Baumgartner et al. (2017).

and weaker magnetic anisotropy compared to Baumgartner et al. (2017). After-pulse relaxation has been observed also in $\mathrm{Ta} / \mathrm{CoFeB} / \mathrm{MgO}$ dots for current pulses exceeding $2 \mathrm{~ns}$, attributed to DW reflection at the sample edges that is favored by the lower DMI and Gilbert damping of $\mathrm{Ta} / \mathrm{CoFeB} / \mathrm{MgO}$ (Yoon et al., 2017). These different results reveal how the reversal path is determined by the balance between DL and FL torques, DMI, magnetic anisotropy, and temperature. For samples matching the width of the current line, the Oersted field can also facilitate or hinder the reversal (Aradhya et al., 2016; Baumgartner et al., 2017). In all cases, however, SOT switching is bipolar and robust with respect to multiple cycling events as well as to the presence of defects.

\section{Switching speed}

One of the most attractive features of SOT switching is the timescale of magnetization reversal. Because the switching speed scales with the lateral dimensions of the sample, and the DW velocity can attain up to 750 m/s (Miron et al., 2011a; Yang et al., 2015), the reversal time can be reduced to well below 1 ns in dots of $100 \mathrm{~nm}$ size (Garello et al., 2014). Figure 32(a) shows that the switching probability of perpendicularly magnetized $\mathrm{Pt} / \mathrm{Co} / \mathrm{AlO}_{x}$ dots has a narrow distribution as a function of pulse length $\tau_{p}$, which decreases to below $100 \mathrm{ps}$ as the current density increases. In such a study, a switching probability of $100 \%$ was demonstrated down to $\tau_{p}=180 \mathrm{ps}$, consistently with reversal due to domain nucleation and propagation. The critical switching current $j_{\mathrm{sw}}$ is characterized by a long and a short time scale regime, shown in Fig. 32(b), similar to STT-induced switching in metallic spin valves (Liu et al., 2014b). $j_{\mathrm{sw}}$ depends weakly on $\tau_{p}$ above $10 \mathrm{~ns}$, as expected for a thermally-activated reversal process (Bedau et al., 2010), and scales linearly with $\tau_{p}^{-1}$ below about $1 \mathrm{~ns}$, as expected in the intrinsic regime where the reversal time is inversely proportional to the transferred angular momentum.

Zhang et al. (2015a) performed a study of how $j_{\mathrm{sw}}$ scales as a function of device size in $\mathrm{Ta} / \mathrm{CoFeB} / \mathrm{MgO}$ [Fig. 32(c,d)]. They observed that $j_{\mathrm{sw}}$ increases by one order of magnitude going from micrometer-sized $\mathrm{Ta} / \mathrm{CoFeB} / \mathrm{MgO}$ stripes to $80 \mathrm{~nm}$ dots, whereas no significant change is observed by further reducing the dot size down to $30 \mathrm{~nm}$. This behavior was interpreted as a signature of incipient monodomain behavior, even though no precessional switching was observed, contrary to the prediction of macrospin models (Lee et al., 2013; Park et al., 2014). An additional feature that makes SOT switching very attractive for applications is that the incubation time required to start the process is negligible (Garello et al., 2014). The SOT geometry, in which $\mathbf{T}_{\mathrm{DL}}$ is orthogonal to the quiescent magnetization, implies that the magnetization reacts immediately to the current, contrary to STT-induced switching, in which $\mathbf{T}_{\mathrm{DL}}$ is initially zero for collinear magnetic layers until thermal fluctuations induce a misalignment of the free layer magnetization that is sufficient to trigger the reversal, leading to ns-long random delays (Devolder et al., 2008; Hahn et al., 2016).

\section{Zero field switching}

A critical issue for perpendicularly magnetized layers is the need to apply an external field $B_{x}$ to uniquely define the switching polarity, as shown in Fig. 30. Although $B_{x}$ by itself cannot switch the magnetization because it is orthogonal to the easy axis, fields ranging from 1 to $100 \mathrm{mT}$ are typically required to achieve deterministic reversal, depending on the current density as well as on 
the magnetic anisotropy of the layers (Avci et al., 2014b). Several approaches have been demonstrated to solve this issue by substituting $B_{x}$ with a real or effective field embedded into a device. The first working concept by Miron et al. (2011b) was to deposit two $50 \mathrm{~nm}$ thick CoFe layers on either side of the magnetic dot, providing a dipolar in-plane field parallel to the current. This solution, however, is not practical for device integration because it limits the scalability of a matrix of such dots or MTJs.

Lau et al. (2016b) have shown that it is possible to embed an in-plane magnetized CoFe layer directly into the stack, and provide an effective $B_{x}$ on the perpendicular $\mathrm{CoFe}$ free layer via interlayer exchange coupling mediated by nonmagnetic Ru or Pt spacers. Such an approach allows for varying the sign of $B_{x}$ upon changing the spacer thickness, but may not be easily integrated into standard MTJ architectures. An alternative solution is to exploit the dipolar field projected by an in-plane magnetized layer placed on top of the free layer/barrier/reference layer stack (Zhao et al., 2017), provided that such a field does not reduce the TMR. A promising route in this respect is to deposit the FM directly on top of a few nmthick AF like IrMn or PtMn (van den Brink et al., 2016; Fukami et al., 2016b; Oh et al., 2016). The AF layer provides an in-plane exchange bias field but also the source of the spin accumulation, which enables the switching of perpendicular FM layers in zero field at current densities
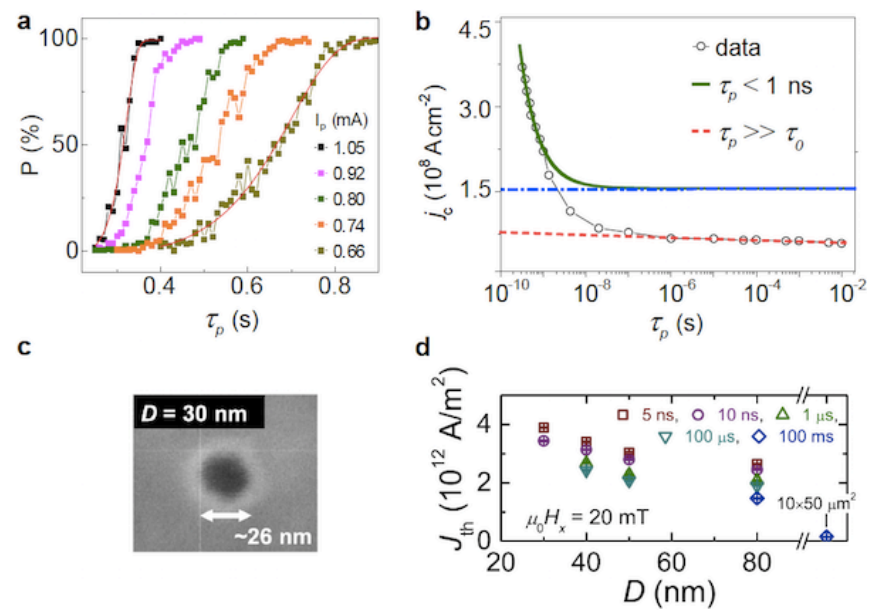

FIG. 32 (Color online) (a) Switching probability $P$ of a square $\mathrm{Pt}(3 \mathrm{~nm}) / \mathrm{Co}(0.6 \mathrm{~nm}) / \mathrm{AlO}_{x}$ dot with a lateral size of $90 \mathrm{~nm}$ as a function of the current pulse duration $\tau_{p}$ at fixed inplane field $B_{x}=91 \mathrm{mT}$. (b) Critical current density as a function of pulse duration defined at $P=90 \%$. The green solid line is a fit to the data in the short-time regime $\left(\tau_{p}<1 \mathrm{~ns}\right)$, the red dashed line is a fit in the thermally activated regime $\left(\tau_{p} \geq 1 \mu \mathrm{s}\right)$. The blue dash-dotted line represents the intrinsic critical current $j_{c 0}$. Adapted from Garello et al. (2014). (c) Scanning electron microscope image of a $\mathrm{Ta}(5 \mathrm{~nm}) / \mathrm{CoFeB}(1.2) / \mathrm{MgO}$ dot with a nominal diameter $D$ of $30 \mathrm{~nm}$. (d) Device diameter dependence of the critical current density at various $\tau_{p}$. Adapted from Zhang et al. (2015a). of the order of $3 \times 10^{7} \mathrm{~A} / \mathrm{cm}^{2}$. The switching process in $\mathrm{FM} / \mathrm{AF}$ systems takes place in a step-wise manner, as schematized in Fig. 33(c), depending on the microstructure of the AF layer and the local direction of the exchange bias field (van den Brink et al., 2016; Fukami et al., 2016b). This behavior can be also exploited to introduce memristive properties into three-terminal MTJ devices, in which the TMR is modulated by the amount of current passed in the FM/AF system (Fukami et al., 2016b).

Finally, an alternative and elegant approach to this problem is to introduce lateral symmetry breaking in the magnetic structure. Thickness gradients of the oxide and FM layers have been shown to induce an out-of-plane FL torque (Yu et al., 2014a,b) or a tilted anisotropy (Torrejon et al., 2015; You et al., 2015), both conducive to zero field switching, whereas independent patterning of the magnetic and conductive layers has been used to control the switching polarity via asymmetric DW propagation (Safeer et al., 2016).

\section{F. Memory and logic devices}

SOT-operated devices can find application in memory as well as logic architectures where current-induced switching is required to control the magnetization of one or several magnetic elements. MTJs with in-plane (Liu et al., 2012b; Pai et al., 2012; Yamanouchi et al., 2013) and perpendicular (Cubukcu et al., 2014) magnetization provide the first demonstration of three-terminal devices in which the write operation is performed by SOTs (Fig. 34). MTJs constitute the building blocks of MRAMs, where the bit state is encoded in the high (low) TMR corresponding to antiparallel (parallel) alignment of the magnetization of the free and reference layers. The ever increasing need for faster data storage and retrieval has placed MRAMs in a prime position to replace or complement CMOS-based memory technologies, especially when performance rather than cost is the primary concern. MRAMs offer nonvolatility, low write energy, low standby power, as well as superior endurance and resistance to radiation compared to semiconductor memories. State-of-the-art MRAMs incorporate STT as the writing mechanism (Kent and Worledge, 2015). STT brings great advantages in terms of scalability and integration with peripheral electronics, since the critical switching current scales with the area of the free layer and requires only two terminals to perform the read and write operations [Fig. 34(a)]. However, as the write and read currents flow along the same path through the oxide tunnel barrier, a compromise between conflicting requirements must be achieved, namely a thin barrier for low current switching and a thick barrier for high TMR. Moreover, because the STT reversal process is thermally activated, a large overdrive current is required for fast 

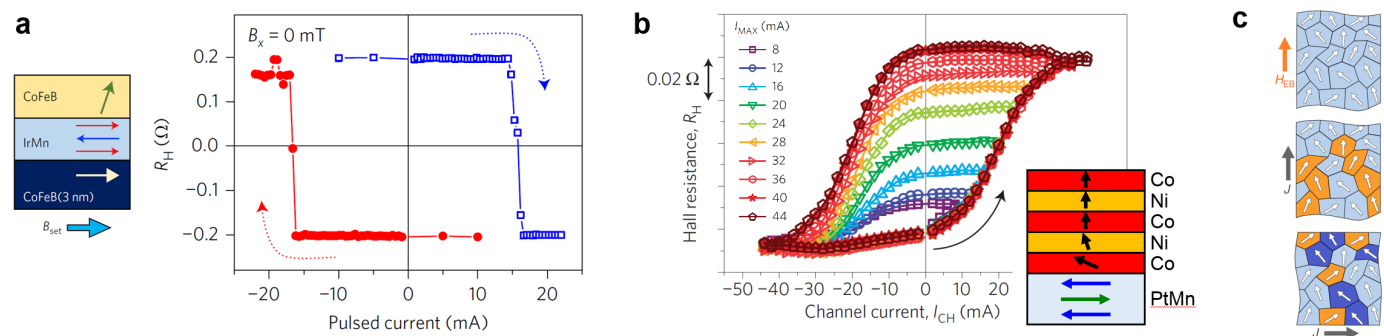

FIG. 33 (Color online) (a) Left: schematic of the in-plane effective field induced by exchange bias. The colored arrows in the layers indicate the direction of the magnetic moments. Field-free switching of $\mathrm{Ta}(5 \mathrm{~nm}) / \mathrm{CoFeB}(3 \mathrm{~nm}) / \mathrm{IrMn}(3 \mathrm{~nm}) / \mathrm{CoFeB} / \mathrm{MgO}$ sample as a function of current (Oh et al., 2016). (b) Magnetization loops of a $[\mathrm{Co}(0.3) / \mathrm{Ni}(0.6)]_{2} / \mathrm{Co}(0.3)$ multilayer on $\operatorname{PtMn}(8 \mathrm{~nm})$ measured after the application of current pulses of increasing amplitude up to the maximum specified in the legend. The black arrow indicates the position from which the measurement starts after initialization by a negative pulse. Adapted from Fukami et al. (2016b). (c) Model representing the uncompensated spin direction in each grain of the AF at the interface with the FM. Top: situation after field-cooling showing an average exchange bias field (orange arrow). A current pulse along the exchange bias direction (middle) or perpendicular to it (bottom) switches the regions of the FM layer coupled to only one type of AF domains. Switched regions are indicated in orange and blocked regions are indicated in dark blue. From (van den Brink et al., 2016).

switching, which can damage the tunnel barrier, while the finite probability to not switch at high currents and to switch at low current leads to write error rates that are larger than desired (Oh et al., 2009). Three-terminal MTJ devices based on SOT offer critical advantages in this respect, as the free layer can be switched without passing a current through the oxide and reference layers [Fig. 34(b)]. The separation of the read and write current paths in the MTJ allows for optimal tuning of the barrier independently of the write process and increases the endurance of the MTJ. Moreover, the deterministic character of SOT switching enables sub-ns reversal of perpendicular MTJs (Cubukcu et al., 2015) and low error rates in in-plane MTJs down to 2 ns long current pulses (Aradhya et al., 2016). Analysis of SOT-MRAMs at the circuit- and architecture-level (Oboril et al., 2015; Prenat et al., 2016) reveals that this technology can be advantageously introduced in the data cache of processors, offering a strong reduction of the power consumption compared to volatile memories and comparable performances to STT-MRAMs, with a slight area penalty due to the three-terminal configuration and significant gains in terms of speed.

SOTs hold great promise also for driving magnetic cellular automata (Cowburn and Welland, 2000), DW logic (Allwood et al., 2005), and MTJ-based logic devices (Guo et al., 2014; Yao et al., 2012). In the first two types of devices, SOTs offer unique features such as the clocking of nanomagnetic logic arrays by in-plane current injection (Bhowmik et al., 2014) and the efficient manipulation of DWs (Safeer et al., 2016; Yang et al., 2015). In hybrid CMOS/magnetic devices based on MTJs, SOTs can perform similar functions as STT (Guo et al., 2014; Yao et al., 2012), but also enable novel architectures. Recent proposals include MTJ devices that exploit gate-voltagemodulated SOT switching for the parallel initialization
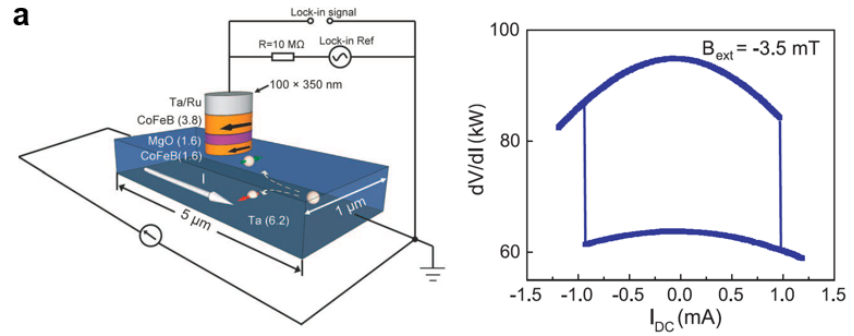

b
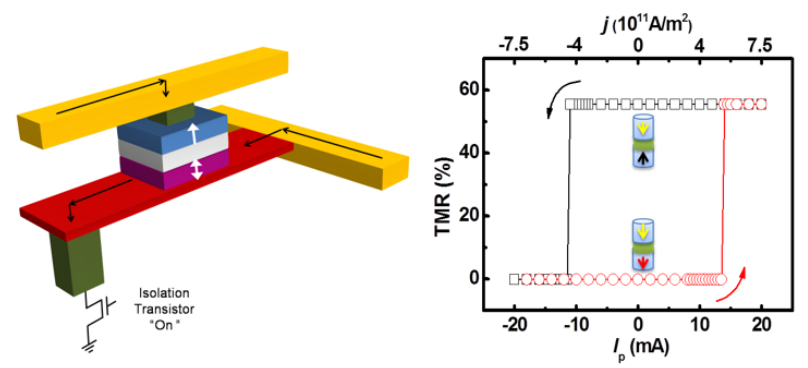

FIG. 34 (Color online) (a) SOT-induced switching for an in-plane magnetized nanomagnet at room temperature: schematic of the three-terminal MTJ device and the circuit used in the measurements. The TMR of the device is shown as a function of applied dc current. An in-plane external field of $3.5 \mathrm{mT}$ is applied to set the device at the center of the minor loop, although this is not required for switching the in-plane magnetized free layer. Adapted from Liu et al. (2012b). (b) Schematic of a three-terminal MTJ with perpendicular magnetization. (b) TMR as a function of current amplitude $I_{p}$ injected in the Ta electrode using $50 \mathrm{~ns}$ long pulses under an in-plane magnetic field of $40 \mathrm{mT}$. Adapted from Cubukcu et al. (2014).

of programmable logic arrays (Lee et al., 2016), four terminal devices that allow for direct cascading at high operation gain and low switching power (Kang et al., 2016), and nonvolatile flip-flops for power gating (Jabeur et al., 2014; Kwon et al., 2014). Other unconventional memory 
and logic architectures can be envisaged based on purely planar structures. In such a case, the SOT would provide the writing mechanism while the reading operation can be performed by the AHE (Moritz et al., 2008) or the unidirectional SMR (Avci et al., 2015a; Olejník et al., 2015; Yasuda et al., 2016).

A critical issue in this wide range of applications is the dynamic power consumption relative to the thermal stability factor of nanomagnets, $\Delta=B_{K} M_{s} V_{F} / 2 k_{B} T$, where $V_{F}$ is the volume of the FM. In perpendicularly magnetized structures with $\Delta \gtrsim 500$, the critical current density ranges from $10^{7}$ to a few times $10^{8} \mathrm{~A} / \mathrm{cm}^{2}$ depending on the switching speed [Fig. 32(b)]. However, because the critical current scales with the lateral crosssection of a device, the switching of a $50 \mathrm{~nm}$ wide dot is predicted to require less than $200 \mu \mathrm{A}$ and a write energy smaller than $100 \mathrm{fJ}$ at $1.5 \mathrm{~ns}$ (Cubukcu et al., 2015), which is close to the best results obtained so far for perpendicular STT-MRAM devices.

Very promising figures of merit in this context have been obtained for in-plane $\mathrm{CoFeB}$ layers with $\Delta \gtrsim 35$ by dusting the $\mathrm{W} / \mathrm{CoFeB}$ interface with $\mathrm{Hf}$, which allows for critical current densities of the order of $5 \times 10^{6} \mathrm{~A} / \mathrm{cm}^{2}$ at 2 ns (Shi et al., 2017). The power dissipated in the current lines is also a matter of concern, as some of the most efficient NM/FM combinations are based on the highresistive phase of $\mathrm{W}$ and $\mathrm{Ta}$ (Liu et al., 2012b; Pai et al., 2012) (Fig. 21). The search for novel SOT materials is thus focusing on systems that combine large charge-spin conversion efficiency with low resistivity or whose magnetic properties can be strongly modulated by a gate voltage. While there are still margins of improvement, SOT devices already offer an unprecedented variety of applications and compatibility with different classes of materials, which extends the range of spintronics well beyond the prototypical spin-valve and MTJ structures of the past two decades.

\section{SPIN-ORBIT TORQUE IN NONCENTROSYMMETRIC MAGNETS}

SHE and iSGE are known as distinct but companion phenomena from their initial observations in non-magnetic semiconductor structures (Belkov and Ganichev, 2008; Ganichev et al., 2004b; Ivchenko and Ganichev, 2008; Kato et al., 2004a,b; Silov et al., 2004; Wunderlich et al., 2004, 2005). As discussed in the previous section, both iSGE and SHE have been utilized for electrically generating SOTs in metallic magnetic multilayers. The primary focus of the present section is to discuss the experiments performed on bulk non-centrosymmetric magnets, including dilute magnetic semiconductors (Chernyshov et al., 2009; Endo et al., 2010; Fang et al., 2011; Kurebayashi et al., 2014), magnetic half-heusler compounds (Ciccarelli et al., 2016) and
AFs (Bodnar et al., 2017; Meinert et al., 2017; Wadley et al., 2016). This type of systems is particularly interesting as SHE is absent (there is no adjacent NM), so that the observed SOTs are solely attributed to iSGE.

In analogy to the galvanic (voltaic) cell, the term spin galvanic effect (SGE) was coined for a phenomenon in which an externally induced non-equilibrium spin polarization generates an electrical current (voltage) (Ganichev et al., 2002). Inversely the iSGE, sometimes also called the Rashba-Edelstein effect, then refers to an externally applied electrical current that generates a spin polarization (Aronov and Lyanda-Geller, 1989; Edelstein, 1990; Inoue et al., 2003; Ivchenko et al., 1989; Ivchenko and Pikus, 1978; Mal'shukov and Chao, 2002). The theory of iSGE was discussed in details in Subsection III.D. We start in Subsection V.A with initial observations of the iSGE in non-magnetic GaAs structures and continue in Subsection V.B by discussing the iSGE induced SOTs in bulk FMs, namely in the low Curie temperature, dilute-moment semiconductor (Ga,Mn)As, and in the high Curie temperature, dense-moment metal NiMnSb. The physics of staggered iSGE spin-polarizations in locally non-centrosymmetric lattices and corresponding Néel SOTs is reviewed in Subsection V.C based on studies in AFic CuMnAs and $\mathrm{Mn}_{2} \mathrm{Au}$. We conclude in Subsection V.E by discussing the SGE and spin-orbit-driven magnonic charge pumping phenomena that are reciprocal to the iSGE and SOT, respectively.

\section{A. Non-magnetic GaAs structures}

Initial observations of the iSGE were made in parallel with the initial SHE experiments, in both cases in semiconductors and employing optical detection methods (Belkov and Ganichev, 2008; Ganichev et al., 2004b; Ivchenko and Ganichev, 2008; Kato et al., 2004a,b; Silov et al., 2004; Wunderlich et al., 2004, 2005). In Ref. (Wunderlich et al., 2004, 2005), iSGE and SHE were detected in the same asymmetrically confined hole gas in a AlGaAs/GaAs semiconductor heterostructure. The experiments are shown in Fig. 35. The current-induced spin-polarization was measured by detecting the circularly polarized electroluminescence from a built-in planar $p$ - $n$ light emitting diode (LED). Since in this semiconductor heterostructure the iSGE has the Rashba symmetry and the corresponding in-plane polarization (perpendicular to the applied electric field) is uniform, the LED was placed across the hole transport channel and an inplane observation angle was used [see Fig. 35(a)]. The measured non-zero circular polarization at zero magnetic field [see Fig. 35(b)] is then a signature of the iSGE spinpolarization of current-carrying holes that radiatively recombined with electrons at the detection LED. For comparison, the SHE experiment is displayed in Figs. 35(c,d). Here opposite out-of-plane spin-polarizations accumulate 
only at the edges and, correspondingly, the detecting LEDs are fabricated along the edges of the transport channel and the emitted light observation angle is outof-plane.

The remarkable strength of these relativistic phenomena was already recognized in the initial experiments performed in the strongly spin-orbit coupled GaAs valence band. The effective iSGE fields inferred from Fig. 35(b) are in Teslas. In other words, the $\sim 1-10 \%$ spin polarization was achieved in the microchip at a $\sim 100 \mu \mathrm{A}$ current, compared to a $\sim 100$ A superconducting magnet that would generate the same degree of spin-polarization in the semiconductor via an external magnetic field. Using Maxwell's equations physics one needs $10^{6} \times$ larger equipment with $10^{6} \times$ larger current than using Dirac equation physics in the iSGE (SHE) microchips to achieve the same polarization in the nominally non-magnetic system.

When the current is switched off these large spinpolarizations immediately vanish, which makes the iSGE and SHE phenomena in non-magnetic crystals impractical for spintronic memory applications. However, shortly after their initial discovery, it was realized theoretically (Bernevig and Vafek, 2005; Garate and MacDonald, 2009; Manchon and Zhang, 2008; Železný et al., 2014) and subsequently verified in experiments (Chernyshov et al., 2009; Ciccarelli et al., 2016; Wadley et al., 2016), that iSGE represents uniquely efficient means for electrical writing of information when the non-equilibrium, spinorbit-induced charge polarizations are exchange-coupled to FM or AF moments. These are discussed in the following subsections.

\section{B. Bulk ferromagnetic (Ga,Mn)As and NiMnSb}

One can picture iSGE based on simple symmetry rules. Fig. 36 represents the iSGE polarizations in three selected systems: (i) Si diamond lattice, (ii) GaAs zincblende crystal and (iii) NiMnSb non-centrosymmetric magnet. A priori, since Si diamond-lattice possesses inversion symmetry, iSGE vanishes globally at the level of the unit cell. But due to the local inversion symmetry breaking, iSGE generates two spin densities, $\mathbf{S}_{A}=$ $-\mathbf{S}_{B}$, pointing in the opposite direction on the two non-centrosymmetric, inversion-partner sites of the $\mathrm{Si}$ diamond-lattice unit cell, as shown in Fig. 36(a). This staggered-symmetry spin density induced by the iSGE can generate an efficient SOT in collinear AFs as further discussed in Subsection V.C.

On the other hand, the zinc-blende lattice of GaAs [or (Ga,Mn)As] and of the closely related half-heusler lattice of NiMnSb are examples of crystals that lack an inversion center in the unit cell. This can result in a non-zero net spin density, illustrated in Figs. 36(b,d), that generates an efficient SOT in FMs, provided that the iSGE-induced

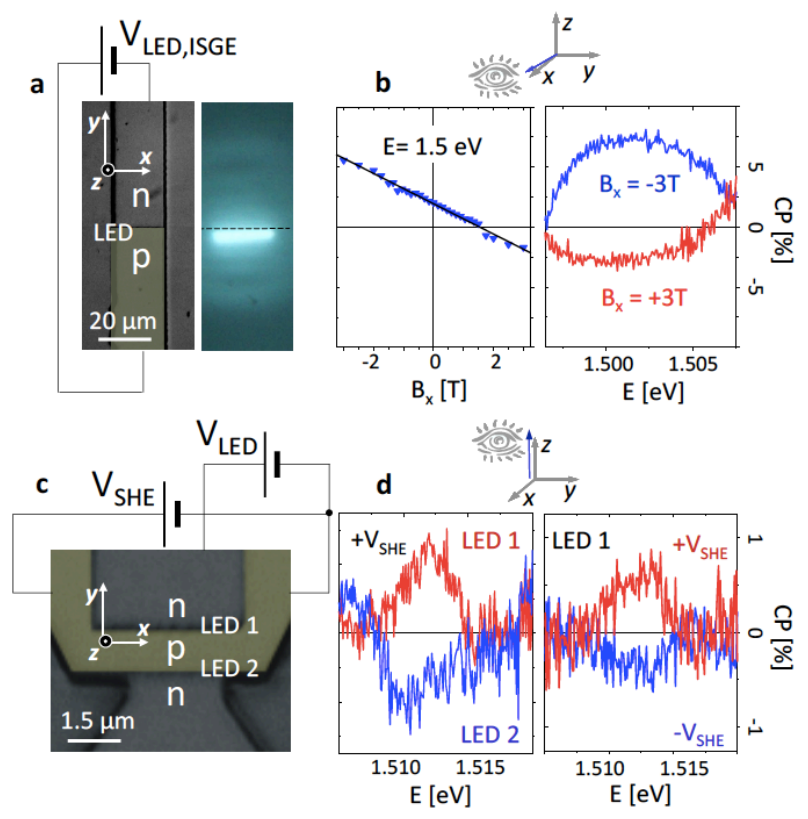

FIG. 35 (Color online) (a) Electron micrograph of the device and an optical image of the emitted light in the experimental detection of the iSGE by circularly-polarized electroluminescence. The uniform in-plane spin polarization is detected by placing the LED across the transport channel and using an in-plane observation angle. (b) Right: spectral dependence of the circular polarization of the emitted light. Left: the dependence of the circular polarization on the external in-plane magnetic field. (c,d) Experimental detection of the SHE by two LEDs placed along the edges of the conduction channel and using an out-of-plane observation angle. Adapted from (Wunderlich et al., 2004, 2005).

spin density is exchange coupled to the FM moments. As discussed earlier in detail in Subsection III.D.1, depending on the crystal symmetry, the iSGE can be composed of three distinct terms: generalized Rashba and Dresselhaus terms, shown in Fig. 36(c), and a term describing a response collinear to the electric field.

The experimental discovery of the iSGE-induced SOT was reported in a $(\mathrm{Ga}, \mathrm{Mn})$ As sample whose image is shown Fig. 37(a) (Chernyshov et al., 2009; Endo et al., 2010). The experiment demonstrated not only the presence of the iSGE effective field of the expected Dresselhaus symmetry for the strained (Ga,Mn)As epilayer, but also demonstrated that iSGE was sufficiently strong to reversibly switch the direction of magnetization. Data in Fig. 37(b) were taken at external magnetic field magnitude and angle fixed close to the switching point between the [010] and [100] easy-axes. The measured transverse AMR, used for the electrical readout, forms a hysteresis loop as the writing iSGE current is swept between $\pm 1 \mathrm{~mA}$. The loop corresponds to the electrical switching between the [010] and [100] easy-axes. Here $100 \mathrm{~ms}$ current pulses of a $1 \mathrm{~mA}$ amplitude and alternating polarity were sufficient to permanently rotate the direction 

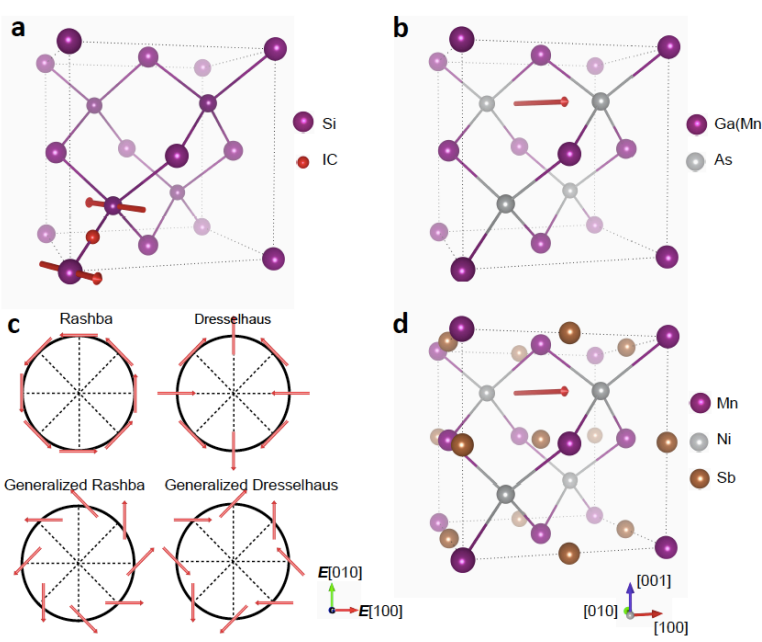

FIG. 36 (Color online) (a) Cartoon representation of opposite iSGE spin-polarizations generated at the locally noncentrosymmetric inversion-partner lattice cites of the Si lattice. (b) Cartoon representation of a net uniform iSGE spinpolarization generated over a non-centrosymmteric unit cell of a zinc-blende GaAs lattice. Exchange coupling between the iSGE spin-polarization of carriers and equilibrium dilute FM moments on Mn atoms results in the SOT. (c) Different symmetries of iSGE spin-polarization as a function of the electric field direction corresponding to different non-centrosymmetric crystal point groups. (d) Same as (b) for a room-temperature dense-moment FM NiMnSb. Adapted from (Ciccarelli et al., 2016).

of magnetization, as highlighted in Fig. 37(c).

A detailed analysis of the magnitude and symmetry of iSGE effective fields in $(\mathrm{Ga}, \mathrm{Mn})$ As was performed by employing an all-electrical ST-FMR technique, sketched in Fig. 38(a) (Fang et al., 2011; Kurebayashi et al., 2014) and presented in Subsection IV.B.2. Here an electric current oscillating at microwave frequencies is used to create an oscillating effective SOT field in the magnetic material being probed, which makes it possible to characterize individual nanoscale samples with uniform magnetization profiles (Fang et al., 2011). For detection, a frequency mixing effect based on the AMR was used. When magnetization precession is driven, there is a time-dependent change $\Delta R(t)$ in longitudinal resistance from the equilibrium value $R$ (owing to the AMR). The resistance oscillates with the same frequency as the microwave current, thus causing frequency mixing, and a directly measurable d.c. voltage $V_{d c}$ is generated. This voltage provides a probe of the amplitude and phase of magnetization precession with respect to the microwave current.

The FMR vector magnetometry on the driving SOT fields revealed a dominant Dresselhaus and a weaker Rashba contribution [Fig. 38(a)] (Fang et al., 2011). By separating the symmetric and antisymmetric parts of the mixing $V_{d c}$ signal [Fig. 38(b)] it was possible to identify both the FL and the DL SOT components (Kurebayashi
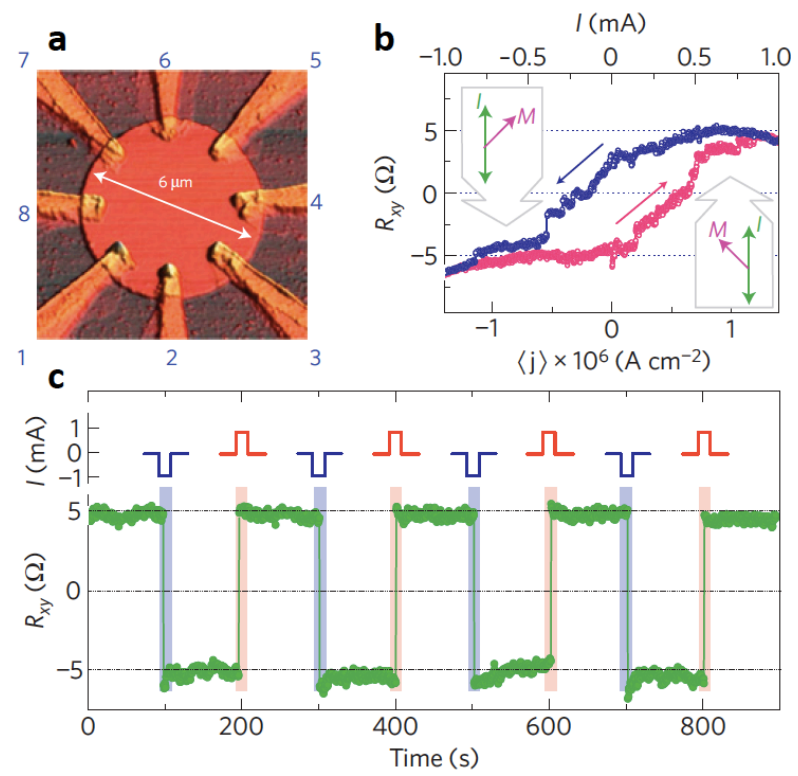

FIG. 37 (Color online) (a) An atomic force micrograph of the sample used to detect the SOT in GaMnAs. (b) $R_{x y}$ shows hysteresis as a function of the current for a fixed external magnetic field $H=6 \mathrm{mT}$ applied at an angle $\phi_{H}=72^{\circ}$. (c) The magnetization switches between the [010] and [100] directions when alternating $\pm 1 \mathrm{~mA}$ current pulses are applied. The pulses have $100 \mathrm{~ms}$ duration and are shown schematically above the data curve. Adapted from (Chernyshov et al., 2009).

et al., 2014). It was shown that the DL SOT plays a comparably important role in driving the magnetization dynamics in $(\mathrm{Ga}, \mathrm{Mn})$ As as the FL SOT [Figs. 38(c,d)].

The FMR technique was also employed in the study of the iSGE-induced SOT in the room-temperature, dense-moment metal FM NiMnSb, as shown in Figs. 39. In agreement with the symmetry expectations for the strained half-heusler lattice of the NiMnSb epilayer, and in agreement with the results in the directly related zincblende lattice of $(\mathrm{Ga}, \mathrm{Mn}) \mathrm{As}$, the observed FL component has a dominant Dresselhaus symmetry [Fig. 39(d)]. Unlike (Ga,Mn)As, the DL SOT was not identified in NiMnSb [Fig. 39(b,c)]. This is likely due to the higher conductivity of metallic NiMnSb. While the extrinsic FL SOT scales with the conductivity, the intrinsic contribution to the DL SOT is scattering-independent to lowest order (see Subsection III.D.1), implying that the higher conductivity of the NiMnSb metal might favor the FL SOT.

\section{Collinear antiferromagnets}

Compensated two-spin-sublattice AF have north poles of half of the microscopic atomic moments pointing in one direction and the other half in the opposite direction. This makes the uniform external magnetic field inefficient 

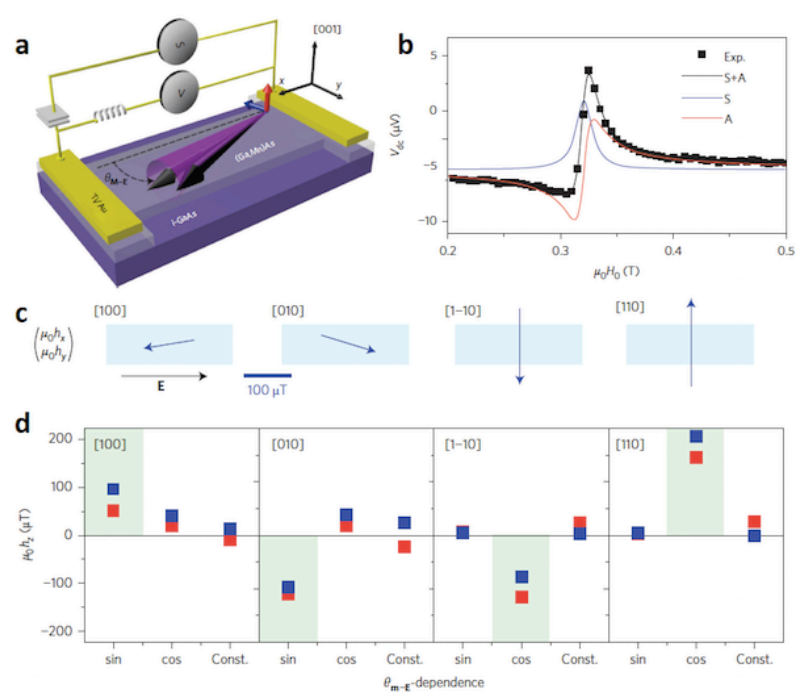

FIG. 38 (Color online) (a) Schematic of the (Ga,Mn)As sample, measurement set-up and magnetization precession. The injected microwave current drives FMR, which is detected via the d.c. voltage $V_{d c}$ across the microbar. $\theta_{\mathbf{m}-\mathbf{E}}$ is the angle of the static magnetization direction measured from the current flow direction. Arrows represent in-plane (blue) and out-ofplane (red) components of the instantaneous non-equilibrium iSGE spin polarization induced by the microwave current that drives the magnetization. (b) A typical spin-orbit FMR signal driven by an alternating current at $11 \mathrm{GHz}$ and measured by $V_{d c}$ as a function of external magnetic field. Data were fitted by a combination of symmetric (S) and antisymmetric (A) Lorentzian functions. (c) Direction and magnitude of the in-plane spin-orbit field (blue arrows) within the microbars (light blue rectangles). The direction of the electric field is represented by $\mathbf{E}$. (d) Coefficients of the $\cos \theta_{\mathbf{m}-\mathbf{E}}$ and $\sin \theta_{\mathbf{m}-\mathbf{E}}$ fits to the angle dependence of the out-of-plane SOT field for the sample set. In this out-of-plane data, two samples are shown in each microbar direction and are distinguished by blue and red square data points. The symmetries expected for the DL SOT, on the basis of the theoretical model for the Dresselhaus spin-orbit Hamiltonian, are shown by light green shading. Adapted from (Kurebayashi et al., 2014).

for switching magnetic moments in AFs. The complete absence of electromagnets or reference permanent magnets in the SOT scheme for writing FM memory bits, discussed above, has served as the key for introducing the physical concept for the efficient control of magnetic moments in AFs (Železný et al., 2014).

Two distinct scenarios can be considered for the SOT on AF spin-sublattices $A / B, \partial_{t} \mathbf{m}_{A / B} \sim \mathbf{m}_{A / B} \times \mathbf{B}_{A / B}^{\mathrm{eff}}$ (Železný et al., 2014). One in which the crystal is globally non-centrosymmetric. Here an example is the halfheusler AF CuMnSb (Forster et al., 1968) or any thin-film AF with structural inversion asymmetry. The efficient torque in this case is the DL SOT which, assuming e.g. Rashba spin-orbit coupling, is driven by an effective field $\mathbf{B}_{A / B}^{\mathrm{eff}} \sim(\mathbf{E} \times \mathbf{z}) \times \mathbf{m}_{A / B}$. Here $\mathbf{B}_{A / B}^{\mathrm{eff}}$ is staggered due to the opposite magnetizations on the two spin-sublattices
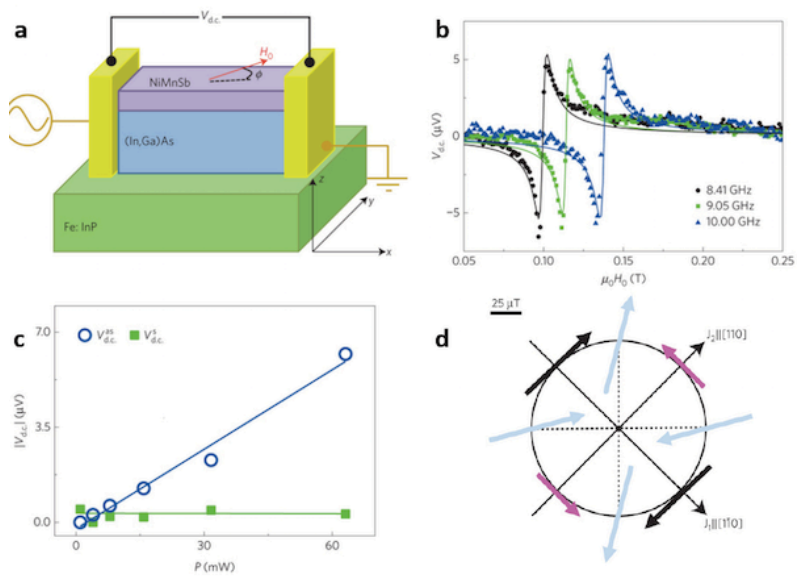

FIG. 39 (Color online) (a) Schematic of the NiMnSb epilayer sample and measuring set-up. A microwave current is passed in the bar and excites spin-orbit FMR. By measuring the longitudinal dc voltage, the magnitude of the spin-orbit driving field is deduced. The angle $\phi$ is the angle between the current flow and the external field around which the magnetisation precesses. (c) The rectified voltage showing FMR for different frequencies of the microwave current. The Lorentzians are well fitted by an antisymmetric line-shape (continuous line) at all frequencies. (d) Power dependence of the symmetric and antisymmetric components of the rectified voltage. Adapted from (Ciccarelli et al., 2016).

of the $\mathrm{AF}, \mathbf{m}_{A}=-\mathbf{m}_{B}$. The FL SOT in these globally non-centrosymmetric crystals in not efficient for AFs since the effective field, $\mathbf{B}_{A / B}^{\text {eff }} \sim \mathbf{E} \times \mathbf{z}$, driving the FL torque is not staggered.

In Fig. 36(a) we illustrated that in crystals with two inversion-partner lattice sites in the unit cell, the iSGE can generate a staggered spin density. This leads to the second scenario in which the FL component of the $\mathrm{SOT}$ is efficient in an $\mathrm{AF}$ whose magnetic spin-sublattices $A / B$ coincide with the two inversion-partner crystalsublattices. In this case the effective field driving the FL SOT has the staggered form (again assuming the Rashba symmetry): $\mathbf{B}_{A}^{\mathrm{eff}} \sim \mathbf{E} \times \mathbf{z}$ and $\mathbf{B}_{B}^{\mathrm{eff}} \sim-\mathbf{E} \times \mathbf{z} . \mathrm{Mn}_{2} \mathrm{Au}$ and CuMnAs are examples of high Néel temperature AF crystals in which this scenario applies (Wadley et al., 2016; Železný et al., 2014).

Fig. 40 illustrates the experimental realization of electrical switching by the staggered SOT field in a CuMnAs memory bit cell (Olejnik et al., 2017a; Wadley et al., 2016). Writing current pulses are sent through the four contacts of the bit-cell to generate current lines in the central region of the cross along one of two orthogonal axes, representing "0" and "1" [Fig. 40(b)]. The writing current pulses give preference to domains with AF moments aligned perpendicular to the current lines (Rashba-like symmetry). Electrical readout is performed by running the probe current along one of the arms of the cross and by measuring the AF transverse AMR across the other arm [Fig. 40(b)]. The write/read functional- 
ity of the CuMnAs memory cells was verified to be not significantly perturbed in a superconducting magnet generating a magnetic field as strong as $12 \mathrm{~T}$ (Wadley et al., 2016). This highlights the efficiency of the staggered SOT fields whose inferred magnitude allowing to switch the $\mathrm{AF}$ moments is only in the $\mathrm{mT}$ range.

The bit-cell write/read signals can be sent at ambient conditions by placing the CuMnAs chip on a standard printed circuit board connected to a personal computer via a 5 V USB interface (Olejnik et al., 2017a). Fig. 40(c) shows an example of data obtained from this proof-ofconcept AF memory device. Apart from demonstrating the application potential of AFs in spintronics thanks to the SOT, it also illustrates a deterministic multi-level switching of the AF bit cell. Here successive $\sim \mu$ s writing pulses along one of the current path directions produce reproducible step-like changes in the memory readout signal. A photoemission electron microscopy study of CuMnAs has associated the multi-level electrical switching signal with the AF moment reorientations within multiple domains (Grzybowski et al., 2017).

The observation of SOT-driven switching has been recently extended to $\mathrm{Mn}_{2} \mathrm{Au}$, where a large AMR ratio up to $6 \%$ is obtained (Bodnar et al., 2017; Meinert et al., 2017). The general switching features are quite similar to the ones observed in CuMnAs, revealing the multidomain magnetic structure of the system. Upon increasing the applied current, the Néel order of the different magnetic domains is progressively reoriented under thermal activation (Meinert et al., 2017), in sharp contrast with the fast switching obtained in NM/FM bilayers driven by DW nucleation/propagation (see Subsection IV.E). This progressive switching seems to be a specific property of $\mathrm{AF}$ materials, as it was also reported in the case of fieldfree switching in AF/FM metallic bilayers (van den Brink et al., 2016; Fukami et al., 2016b; Oh et al., 2016) (see Subsection IV.E.3).

The multi-level nature of AF bit cells allows to combine memory and logic functionalities (e.g., pulse-counter) within the cell (Olejnik et al., 2017a). Another unique merit of AFs is the THz scale of the internal spin dynamics which in combination with the SOT physics opens the door to ultra-fast switching schemes. Fig. 40(d) shows initial results of experiments in this direction demonstrating a deterministic memory-counter functionality for $\sim$ 1000 pulses with individual pulse-length scaled down to 250 ps. In these experiments, current pulses were delivered via wire-bonded contacts for which pulse-length $\sim 100 \mathrm{ps}$ is at the limit achievable with common currentpulse setups.

Subsequently, reversible switching with analogous characteristics was demonstrated using 1 ps long writing pulses (Olejnik et al., 2017b). A non-contact technique was employed for generating the ultra-short current pulses in the AF memory cell via $\mathrm{THz}$ electromagnetic transients to overcome the above limit of common contact current-pulse setups. Remarkably, the writing energy did not increase when down-scaling the pulse-length from ns to ps. This is in striking contrast to FM STT (Bedau et al., 2010) or SOT (Garello et al., 2014) memories in which the theoretically extrapolated writing energy at ps would increase by three orders of magnitude compared to the state-of-the-art ns-switching devices. While readily achievable in AFs, the ps range remains elusive for FMs because, in frequency terms, it far exceeds the GHz-scale of the FMR in typical FMs.
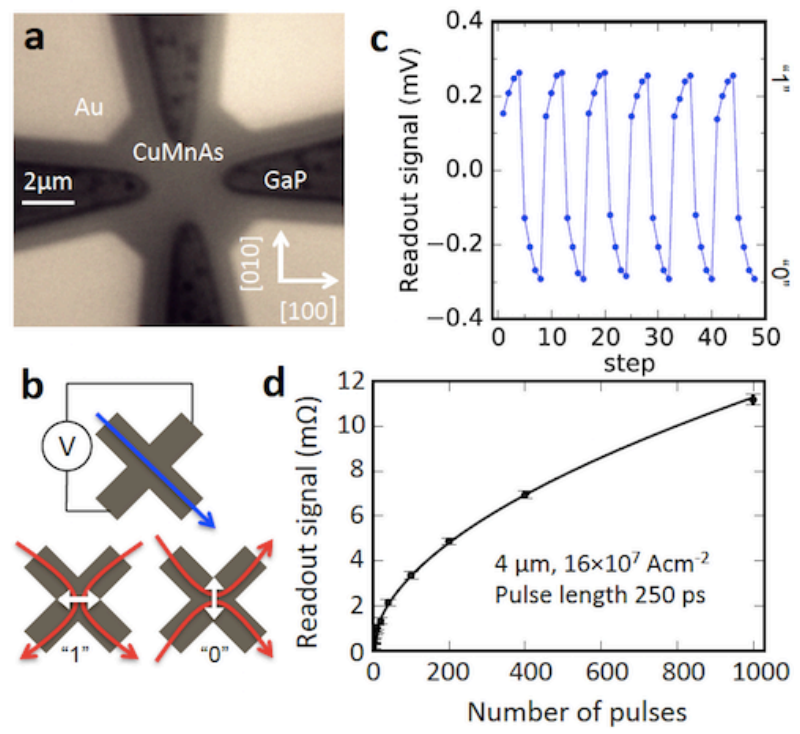

FIG. 40 (Color online) (a) Optical microscopy image of the device containing Au contact pads and the AF CuMnAs crossshape cell on the GaP substrate. (b) Top: The readout current (blue arrow) and transverse voltage detection geometry. Bottom: Write pulse current lines (red arrows) labeled "1" (left) and "0" (right) and the corresponding preferred AF moment orientations (white double-arrows). (c) Readout signals after repeated four write pulses with current lines along the [100] direction ("0") followed by four pulses with current lines along the [010] direction ("1"). (d) Readout signal as a function of the number of pulses in the train of pulses for the individual pulse length of 250 ps. Adapted from (Olejnik et al., 2017a).

\section{Antiferromagnetic topological Dirac fermions}

Recently, a new concept has been theoretically proposed. It follows from the observation that the staggered SOT fields can co-exist with topological Dirac fermions in the band structure of AFs because of the serendipitous overlap of the key symmetry requirements (Šmejkal et al., 2017). Therefore, one can use SOT to reorient the Néel vector in AFs in order to control such topological Dirac fermions. This is illustrated in Fig. 41(a,b) on examples of the CuMnAs where the SOT switching was experimentally verified, as mentioned above, and 
of the graphene lattice representing the Dirac systems (Castro Neto et al., 2009): (i) The two-Mn-site primitive cell of CuMnAs favors band crossings in analogy with the two-C-site graphene lattice. (ii) In the paramagnetic phase, CuMnAs has time reversal $(\mathcal{T})$ and space inversion $(\mathcal{P})$ symmetries. It guarantees that each band is double-degenerate forming a Kramer's pair, in analogy to graphene. In the AF phase, this degeneracy is not lifted because the combined $\mathcal{P} \mathcal{T}$ symmetry is preserved, although the $\mathcal{T}$ symmetry and the $\mathcal{P}$ symmetry are individually broken (Chen et al., 2014; Herring, 1966; Šmejkal et al., 2017; Tang et al., 2016). (iii) The combined $\mathcal{P} \mathcal{T}$ symmetry is just another way of expressing that the two AF spin-sublattices conincide with the two inversion-partner crystal-sublattices. As explained above, this is the condition that allows for the efficient FL SOT in AFs.

An additional crystal symmetry is needed to mediate the dependence of Dirac quasiparticles on the Néel vector orientation [Fig. 41(c)]. In graphene there is no symmetry that protects the four-fold degeneracy of Dirac crossings of two Kramer's pair bands in the presence of spinorbit coupling (Kane and Mele, 2005). In CuMnAs, on the other hand, the Dirac crossings are protected by a non-symmorphic, glide mirror plane symmetry (Young and Kane, 2015), $\mathcal{G}_{x}=\left\{\mathcal{M}_{x} \mid \frac{1}{2} 00\right\}$, as long as the Néel vector is aligned with the [100] axis. $\mathcal{G}_{x}$ combines the mirror symmetry $\mathcal{M}_{x}$ along the (100)-plane with the halfprimitive cell translation along the [100] axis [Fig. 41(d)]. Due to the mirror-reflection behavior of the axial vectors of magnetic moments [Fig. 41(e)], the $\mathcal{G}_{x}$ symmetry, and thus also the Dirac crossing protection, is broken when the AF moments are reoriented into a general crystal direction by the SOT.

\section{E. Magnonic charge pumping in $(\mathrm{Ga}, \mathrm{Mn}) \mathrm{As}$}

We conclude this section by briefly discussing the spin galvanic effect (SGE), which is a reciprocal phenomenon to the iSGE, and its counterpart in magnets termed the magnonic charge pumping (Ciccarelli et al., 2014). The latter, in turn, is a reciprocal phenomenon to the SOT. Following theoretical predictions (Aronov and LyandaGeller, 1989; Edelstein, 1990; Inoue et al., 2003; Ivchenko et al., 1989; Ivchenko and Pikus, 1978; Mal'shukov and Chao, 2002), the SGE was initially observed in an asymmetrically confined GaAs quantum well (Ganichev et al., 2002). The key signature of the SGE is the electrical current induced by a non-equilibrium, but uniform, polarization of electron spins. In the non-equilibrium steadystate, the spin-up and spin-down sub-bands have different populations, induced in the Ganichev et al., 2002 experiment by a circularly polarized light excitation. Simultaneously, the two sub-bands for spin-up and spindown electrons are shifted in momentum space due to
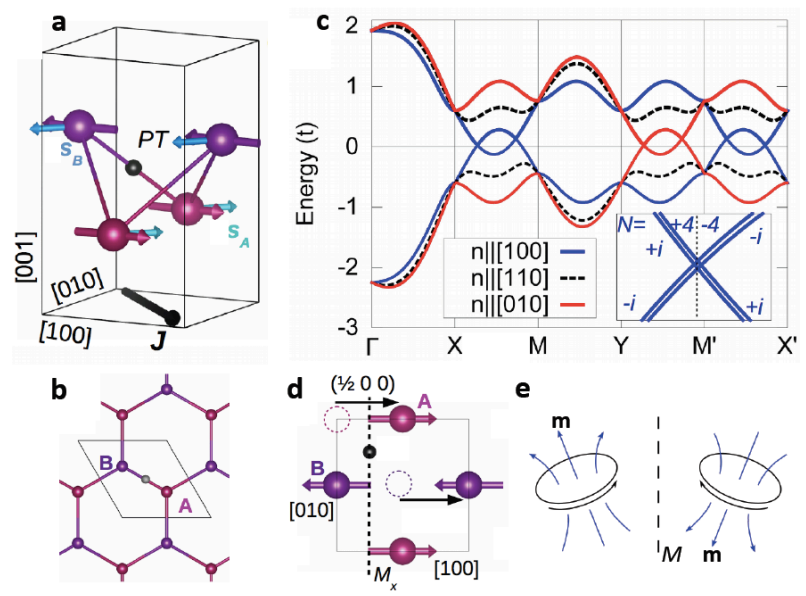

FIG. 41 (Color online) (a) Mn AF spin-sublattices of CuMnAs denoted by purple and pink balls with thick arrows. The AF order breaks time-reversal symmetry $(\mathcal{T})$ and spaceinversion symmetry $(\mathcal{P})$, however, the combined $\mathcal{P} \mathcal{T}$ symmetry is preserved. Staggered current-induced spin-polarization on the sublattices $A$ and $B$ is denoted by cyan and blue arrows. (b) Graphene crystal with two C-sites per unit cell in analogy with the Mn-sites in CuMnAs. (c) Band dispersion of the minimal AF model based on CuMnAs illustrating the control of the Dirac points by the direction of the Néel vector n. Topological indices of the Dirac point are shown in the inset (for the sake of clarity the degenerate bands are slightly shifted). (d) Top view of the model quasi-2D-AF lattice of CuMnAs highlighting the non-symmorphic glide mirror plane symmetry, combining mirror plane $\left(\mathcal{M}_{x}\right)$ reflection with a half-unit-cell translation along the $x$-axis. (e) An axial vector $\mathbf{m}$ under mirror $(\mathcal{M})$ reflection. Adapted from (Šmejkal et al., 2017).

the inversion asymmetry of the semiconductor structure which leads to an inherent asymmetry in the spin-flip scattering events between the two sub-bands. This results in the flow of the electrical current.

The Onsager reciprocity relations imply that there is also a reciprocal phenomenon of the iSGE induced SOT in which electrical signal due to the SGE is generated from magnetization precession in a uniform, spin-orbit coupled magnetic system with broken space inversion symmetry (see Fig. 42) (Hals et al., 2010; Kim et al., 2012a; Tatara et al., 2013). In this reciprocal SOT effect no secondary spin-charge conversion element is required and, as for the SOT, (Ga,Mn)As with broken inversion symmetry in its bulk crystal structure and strongly spinorbit coupled holes represents a favorable model system to explore this phenomenon. The effect was observed in ( $\mathrm{Ga}, \mathrm{Mn}) \mathrm{As}$ and termed the magnonic charge pumping (Ciccarelli et al., 2014). This effect is physically similar to the SGE (or alternatively called inverse RashbaEdelstein effect) observed at $\mathrm{Bi} / \mathrm{Ag}(111)$ (Rojas-Sánchez et al., 2013) or topological insulators surfaces (Shiomi et al., 2014). 
a
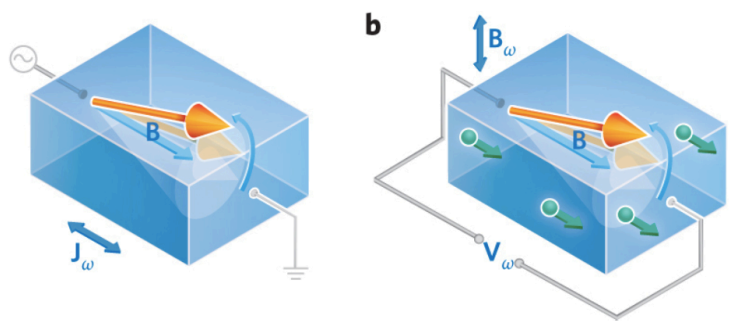

FIG. 42 (Color online) (a) A charge current through ( $\mathrm{Ga}, \mathrm{Mn})$ As results in a non-equilibrium spin polarization of the carriers, which exchange-couples to the magnetization and exerts the SOT. An alternating current generates a timevarying torque, which drives magnetic precession resonantly when a magnetic field is applied. (b) The reciprocal effect of (a) termed the magnonic charge pumping. From Ref. Ciccarelli et al., 2014.

\section{SPIN-ORBIT TORQUES AND MAGNETIC TEXTURES}

The electrical manipulation of magnetic textures using SOTs opens stimulating perspectives for applications. In Section IV, we already mentioned that DW nucleation and propagation play an important role in the context of SOT-driven switching. In addition, intentional and well-controlled DW manipulation constitutes the basis of alternative, DW-based racetrack memories (Fert et al., 2013; Parkin and Yang, 2015; Parkin et al., 2008) and logic concepts (Allwood et al., 2005; Zhang et al., 2015d). In this context, a major breakthrough has been the recent realization and control of individual metastable skyrmions at room temperatures, which shows promising potential for such applications (Fert et al., 2017; Jiang et al., 2017a). Nonetheless, evaluating SOTs in magnetic textures poses a specific challenge compared to the magnetically uniform thin films discussed in Section IV. While SOTs induce a rotation of the magnetization that can be 'simply' recorded through magnetometry (AMR, AHE or MOKE), in magnetic textures one can only evaluate the global impact of the SOTs through the texture motion and deformation. This feature transforms the magnetometry issue to a magnetic microscopy issue. The present section addresses SOT-driven DW and skyrmion motion and dynamics in detail.

Starting with a phenomenological description of the influence of current-induced torques on DW motion in Subsection VI.A, we then discuss its experimental observation in in-plane and perpendicularly magnetized DW in Subsections VI.B and VI.C, respectively. Recent progress achieved on ferrimagnetic and AF systems is presented in Subsection VI.D. The role of DW nucleation and propagation in SOT driven switching is discussed in Subsection IV.E

\section{A. Domain wall dynamics under current}

The dynamics of magnetic textures is governed by the Landau-Lifshitz-Gilbert (LLG) equation at the basis of the continuous theory of magnetic structures, called micromagnetics (Hubert and Schäfer, 1998). In this framework, the local magnetization vector is written $\mathbf{M}(\mathbf{r}, t)=$ $M_{\mathrm{s}} \mathbf{m}(\mathbf{r}, t)$, where the spontaneous magnetization modulus $M_{\mathrm{s}}$ depends on temperature, whereas the unit vector $\mathbf{m}$ specifies its local orientation as a function of space and time. The general LLG equation is

$$
\partial_{t} \mathbf{m}=-\gamma \mathbf{m} \times \mathbf{B}_{\mathbf{M}}+\alpha \mathbf{m} \times \partial_{t} \mathbf{m}+\left(\gamma / M_{\mathrm{s}}\right) \mathbf{T},
$$

where $\gamma>0$ is the (absolute value of the) gyromagnetic ratio $\left(1.76 \times 10^{11} \mathrm{~s}^{-1} \cdot \mathrm{T}^{-1}\right.$ for free electrons), and $\alpha$ the Gilbert damping parameter (no dimensions). The dynamics is driven by the effective field introduced by Landau and Lifshitz as the (functional) derivative of energy density $\mathcal{E}$ versus magnetization $\mathbf{B}_{\mathbf{M}}=-\delta \mathcal{E} / \delta \mathbf{M}$, and by other torques $\mathbf{T}$ that may not derive from an energy density, notably the torques induced by an electrical current. These are of two forms. On the one hand, the STT is generally written, in its local version, as the sum of so-called adiabatic and non-adiabatic terms (Beach et al., 2008)

$$
\left(\gamma / M_{\mathrm{s}}\right) \mathbf{T}_{\mathrm{STT}}=-(\mathbf{u} \cdot \nabla) \mathbf{m}+\beta \mathbf{m} \times[(\mathbf{u} \cdot \nabla) \mathbf{m}],
$$

where the velocity $\mathbf{u}$ is proportional to the electrical current density in the magnetic material, its spin polarization etc., and where $\beta$ is the non-adiabaticity factor (no dimensions). This torque is proportional to the gradient of magnetization along the current direction and thus vanishes in the domains. On the other hand, the SOT is expressed by Eq. (1). The SOT does not depend on the gradient of the magnetization at the lowest order, hence acts also on the magnetization within the domains [for higher order expansion, see van der Bijl and Duine (2012)]. Note that in general when a current is applied to a magnet/metal bilayer, it flows both into the magnet, leading to STT, and into the metal, leading to SOT in the magnet as well as to an Oersted field. We thus need to study the effect of these three torques on DWs.

A qualitative analysis of these two forms of currentinduced torques is instructive. For this, we consider the effective field $\mathbf{B}$ obtained by writing $\mathbf{T}=\mathbf{M} \times \mathbf{B}$, the evaluation being performed at the center of the DW. In order to get a steady current-induced DW motion (CIDM) under a torque $\mathbf{T}$, one basically needs this effective field to be directed along the domains magnetization (a subtlety exists for the vortex wall, as the magnetization is not uniform outside the vortex core, see below). Therefore, we need to know the possible types of magnetic DWs in samples where large current pulses (typical current density $10^{11} \mathrm{~A} / \mathrm{m}^{2}$ ) can be applied. In order to promote large current densities while avoiding excessive sample Joule heating, these samples have the shape of 
(a): X domains

\begin{tabular}{|c|c|c|c|}
\hline TW & $\rightarrow$ & $\uparrow$, & \\
\hline \multicolumn{4}{|c|}{ (b): $Z$ domains } \\
\hline BW & $\odot$ & $\uparrow$ & $\otimes$ \\
\hline \multicolumn{4}{|c|}{ (c): Y domains } \\
\hline Y-NW & & $\rightarrow \quad$ & \\
\hline
\end{tabular}
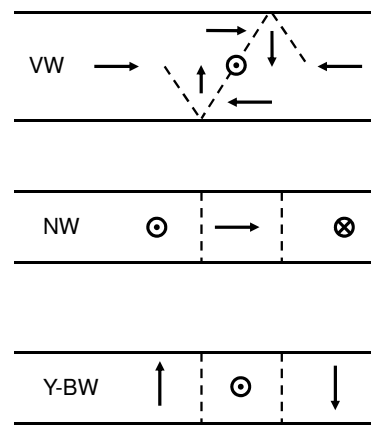

FIG. 43 Schematic of DW structures in nanostrips relevant for SOT studies. (a) When the magnetic easy axis is along the nanostrip ( $x$ axis), typically for small magnetic anisotropy, magnetostatics leads to two basic structures, the transverse wall (TW) at small width and thickness, and the vortex wall (VW) at larger lateral dimensions (McMichael and Donahue, 1997; Nakatani et al., 2005). Note that these DWs have a non-zero magnetostatic charge. (b) When the easy axis is perpendicular ( $z$ axis), typically for strong interface anisotropy, magnetostatics favors the Bloch wall (BW) but the interfacial DMI can favor the Néel wall (NW) (Heide et al., 2008; Thiaville et al., 2012), and fix its chirality. A 'bulk' DMI would favor the BW, and fix its chirality. (c) The last case of a transverse easy axis ( $y$ axis) is rare. The associated walls, known for a long time (Hubert and Schäfer, 1998), are the Néel wall (Y-NW) at small thickness, and the Bloch wall (Y-BW) at larger thickness. In the absence of DMI, the DW magnetization is uncorrelated to the magnetization in the domains, so that domain or DW magnetization arrows can be reversed with no change of energy. The dashed lines outline the shape of the DWs.

nanostrips, with a width $w$ of about a few hundreds of nanometers, and a thickness $h$ of the order of a few nanometers (the thickness being generally thinner for interfacial SOT). As shown in Fig. 43, a limited number of DW structures has to be considered, according to the magnetic anisotropy of the sample.

The STT torques, Eq. (64), depend on the magnetization gradient along the current direction, i.e., $\sim(\mathbf{u} \cdot \boldsymbol{\nabla}) \mathbf{m}$ ( $\equiv \partial_{x} \mathbf{m}$ with the axes convention defined in Fig. 43). From Fig. 43 we see that at the DW center this derivative is along the magnetization of the domain on the right of the DW (an exception to this rule is afforded by the VW, where the magnetization streamlines are reoriented by $90^{\circ}$ through the vortex structure). By construction, the effective field $\mathbf{B}$ associated with the adiabatic STT is orthogonal to the domains magnetization, so that it cannot lead to steady DW motion. On the other hand, the effective field associated with the non-adiabatic STT lies along the domain magnetization. This explains qualitatively the rule for steady STT driven DW motion, given by the velocity formula $\mathbf{v}=(\beta / \alpha) \mathbf{u}$ in which DWs move along the carriers for positive current polarization (majority spin polarization of the current) and positive $\beta$ factor (Thiaville et al., 2005; Zhang and Li, 2004). The

same conclusions are reached for the VW case, by considering the surrounding of the vortex core instead of the domains.

We now perform the same analysis for the SOTs. The effective field associated with the FL SOT reads $\mathbf{B}_{\mathrm{FL}}=$ $\left(\tau_{\mathrm{FL}} / M_{\mathrm{S}}\right) \boldsymbol{\zeta}$ with $\boldsymbol{\zeta} \| \mathbf{y}$ for a current along $x$, considering the Rashba symmetry of the spin-orbit coupling. This field is oriented like the main part of the Oersted field (as $w \gg h$ the $y$ component of the stray field dominates the $z$ component). The results for the various DW structures are summarized in Table III, a generalization of those of Khvalkovskiy et al., 2013: apart from the obvious case of $y$ easy axis (Obata and Tatara, 2008), no steady DW motion is expected. On the other hand, for the DL SOT with $\mathbf{B}_{\mathrm{DL}}=\left(\tau_{\mathrm{DL}} / M_{\mathrm{s}}\right) \mathbf{m} \times \boldsymbol{\zeta}$, only the Néel wall $(\mathrm{NW})$ for the $z$ easy axis is expected to be set in steady motion.

In the cases where the above analysis predicts no steady DW motion, the effective field $\mathbf{B}$ associated to each considered current-induced torque term is not oriented along the domain magnetization (the domain-like magnetization adjacent to the vortex core on the positive $x$ side, for the $\mathrm{VW}$ ). When this field is orthogonal to the DW magnetization it leads to a deformation of the DW structure, that increases with current density. The theory of magnetic DW motion (Hubert and Schäfer, 1998) has shown that a DW structure can accommodate a dynamic deformation up to a certain limit. In the well-known case of field-induced DW motion, this deformation results from the progressive rotation of the magnetization around the applied field, and steady DW motion ceases when the field reaches the so-called Walker field beyond which the dynamic DW structure features a continuously precessing DW magnetization. By very general arguments initially due to Slonczewski (1972) according to which the DW position and the angle of the DW magnetization are coupled variables in the Hamilton sense, a continuously precessing DW magnetization creates a DW velocity. In the field-driven case, this additional velocity opposes that due to the applied field, hence the term of Walker breakdown stressing that DW velocity drops above the Walker field. For STT driven motion, however, the velocity increases in the non-steady regime when $\beta<\alpha$ (Thiaville et al., 2005; Zhang and Li, 2004). We therefore have a second type of motion that is due to fields $\mathbf{B}$ that do not give rise to steady motion, as soon as they are not zero.

If these fields are below the 'breakdown' threshold, a DW position shift will appear as a result of the DW structure transformation when current is applied. When current goes back to zero, and provided the sample is perfect, the opposite DW position shift will however occur as the DW recovers its initial structure. Note that several devices based on an anticipated stick-slip DW motion under application of dissymmetric pulses with short rise-time and long fall-time have been proposed, based on this phenomenon. A partial list of cases with DW 


\begin{tabular}{|c|c|c|c|c|}
\hline DW & STT ad. & T na. & $\begin{array}{c}\text { FL SOT/ } \\
\text { Oersted }\end{array}$ & L SOT \\
\hline TW & $\begin{array}{c}\mathrm{N} \\
\text { odd }\end{array}$ & $\mathrm{Y}$ & $\begin{array}{c}\mathrm{N} \\
\text { even }\end{array}$ & $\begin{array}{c}\mathrm{N} \\
\text { null }\end{array}$ \\
\hline VW & $\begin{array}{c}\mathrm{N} \\
\text { odd }\end{array}$ & $\mathrm{Y}$ & $\begin{array}{c}\mathrm{N} \\
\text { even }\end{array}$ & $\begin{array}{c}\mathrm{N} \\
\text { odd }\end{array}$ \\
\hline BW & $\begin{array}{c}\mathrm{N} \\
\text { odd }\end{array}$ & $\mathrm{Y}$ & $\begin{array}{c}\mathrm{N} \\
\text { even }\end{array}$ & $\begin{array}{c}\mathrm{N} \\
\text { null }\end{array}$ \\
\hline NW & $\begin{array}{c}\mathrm{N} \\
\text { odd }\end{array}$ & $\mathrm{Y}$ & $\begin{array}{c}\mathrm{N} \\
\text { even }\end{array}$ & $\bar{Y}$ \\
\hline Y-NW & $\begin{array}{c}\mathrm{N} \\
\text { odd }\end{array}$ & $\mathrm{Y}$ & $\mathrm{Y}$ & $\begin{array}{c}\mathrm{N} \\
\text { odd }\end{array}$ \\
\hline Y-BW & $\begin{array}{c}\mathrm{N} \\
\text { odd }\end{array}$ & $\mathrm{Y}$ & $\mathrm{Y}$ & $\begin{array}{c}\mathrm{N} \\
\text { odd }\end{array}$ \\
\hline
\end{tabular}

TABLE III Characteristics of the effective field $\mathbf{B}$ assocciated with the current-induecd torques, evaluated at the center of the DW types shown in Fig. 43. For each case, the first line indicates $(\mathrm{Y} / \mathrm{N})$ is this effective field drives or not the DW into steady motion. When it does not, the second line indicates (null/odd/even) if this field is zero and, when it is not, if it is even or odd with respect to the DW magnetization.

shift was presented in Khvalkovskiy et al., 2013. The full list is given in Table III. Finally, when the effective field related to a current-induced torque is large enough, the DW structure will go to its image where some DW magnetization components have been reversed. Whether this process continues or not depends on the power to which the DW magnetization enters the expression of the effective field $\mathbf{B}$. If this power is odd, the opposite field will act on the opposite DW magnetization, leading to indefinite precession of DW magnetization and hence to long-term DW motion, called precessional DW motion. If the power is even, however, indefinite precession will not occur and only a DW position shift will occur. These cases are also indicated in Table III. The table shows that FL SOT (and Oersted field) can only drive DWs in the $y$-easy axis situation, see Y-DWs in Fig. 43.

With this analysis in mind, we turn in the next subsections to each situation, reviewing the experimental reports existing on the subject.

\section{B. In-plane magnetized samples}

\section{Soft samples ( $X$ domains)}

These samples have been the workhorse of the initial studies of the STT, leading to the definition of the adiabatic and non-adiabatic STT terms. As Table III shows, such samples are generally not adequate to test the SOT. The VW is a special case in this picture, being a composite object that can easily deform by lateral motion of the vortex core, inducing a displacement of the whole wall along the nanostrip [see e.g., (Beach et al., 2008; Clarke et al., 2008; Tretiakov et al., 2008)]. As a result, under adiabatic STT for example, the vortex core displaces laterally (along $y$ ), leading to a longitudinal DW displacement (along $x$ ). The effect is however transient as the core eventually stops or disappears at the nanostrip edge, transforming the $\mathrm{VW}$ into a transverse wall (TW). The same effect is expected under SOT.

Up till now, only two studies have considered X domains with adjacent heavy metal layers. An earlier study on Pt/NiFe (Vanhaverbeke et al., 2008) investigated the influence of the current direction on the DW polarity (i.e. the direction of the DW's transverse magnetization), and another more recent study addressed thermal effects in $\mathrm{Ta} / \mathrm{NiFe} / \mathrm{Pt}$ (Torrejon et al., 2012). Moreover, typical thicknesses of the FM film were $10 \mathrm{~nm}$, so that the effect of the interfacial torques is strongly reduced. Note that the Oersted field effect was directly observed in the case of a bilayer sample (Uhlír et al., 2011) by time-resolved photoelectron emission microscopy using $\mathrm{x}$ ray magnetic circular dichroism (XMCD-PEEM), a technique that could be used to measure the FL SOT in situ. Simulations have shown that FL SOT modifies the STT driven dynamics (Seo et al., 2012).

Trilayer samples, typically $\mathrm{Co} / \mathrm{Cu} / \mathrm{NiFe}$ where easier DW motion and higher velocities have been observed, are a special case that could not be understood in the frame of STT plus Oersted fields. It was thus proposed that perpendicular spin currents may play some role (Pizzini et al., 2009; Uhlír et al., 2010). Khvalkovskiy et al. (2009) performed a numerical exploration of the effect of various forms of SOT on both TW and VW, taking $\zeta=\mathrm{x}$ and $\boldsymbol{\zeta}=\mathbf{z}$, i.e. the two cases that are not considered in standard SOT configuration [the latter case was investigated in (Khvalkovskiy et al., 2013) for TW]. The results show that indeed in some cases DW sustained motion is expected (FL SOT for $\boldsymbol{\zeta}=\mathrm{x}$, DL SOT for $\boldsymbol{\zeta}=\mathbf{z}$ for a $\mathrm{VW}$ ), but their relation to the experimental situation is unclear. Another family of bilayer samples are the synthetic AFs. In $\mathrm{CoFe} / \mathrm{Ru} / \mathrm{CoFe}$, a very low threshold for CIDM has been measured (Lepadatu et al., 2017), and attributed to the intrinsic dynamics of antiferromagnetically-coupled TWs, driven by nonadiabatic STT (see Subsection VI.D).

\section{Anisotropic samples with $Y$ domains}

In the case of Y-DWs (see Fig. 43) the FL SOT is directly active (Obata and Tatara, 2008). Such samples require an in-plane anisotropy that is stronger than the magnetostatic energy cost. This has been realized by growing epitaxial layers on single-crystal substrates. One example is $(\mathrm{Ga}, \mathrm{Mn})$ As grown on (001) GaAs (Thevenard et al., 2017), where structures with $\mathrm{X}$ domains and $\mathrm{Y}$ domains were compared, on $50 \mathrm{~nm}$ thick layers so that 
bulk SOT would be active. Large current-induced effects were observed, that strongly differed in the two cases, but no simple and global understanding of the observed effects could be found.

Another way to obtain such structures is to use large magnetostriction materials, as growth-induced stress is relaxed at the edges of a nanostrip, modifying the anisotropy locally. As a result, transverse $\mathrm{Y}$ domains were observed in $\mathrm{Ni}_{80} \mathrm{Pd}_{20}$ films (Chauleau et al., 2011). No study of CIDM could however be realized on such samples, as the Curie temperature was rapidly reached.

\section{Perpendicularly magnetized samples}

1. Demonstrations of spin-orbit torques in current-induced domain wall motion

As spin torque theories predicted that the nonadiabatic torque might be larger in narrow DWs, materials with perpendicular anisotropy appeared as a promising route towards more efficient CIDM. Numerous studies focused on the influence of the electric current on the DW depinning (Boulle et al., 2008; Burrowes et al., 2010; Ravelosona et al., 2005). The results seemed encouraging, but there were only few systems exhibiting CIDM without the assistance of external field. One of these systems were the $\mathrm{Co} / \mathrm{Ni}$ multilayers where the predictions of the adiabatic STT model were most clearly evidenced (Koyama et al., 2011): (i) the existence of an intrinsic critical current that depends on the geometric structure of the DW rather than the extrinsic pinning; (ii) the independence of the critical current on a perpendicular magnetic field.

Among the materials with perpendicular anisotropy, the $\mathrm{Pt} / \mathrm{Co} / \mathrm{AlOx}$ trilayers in particular have attracted a lot of interest. The DW motion was found to be significantly faster (Baumgartner et al., 2017; Miron et al., 2011b; Moore et al., 2008) compared to the previous observations in $\mathrm{NiFe}$ or $\mathrm{Co} / \mathrm{Ni}$ films [Fig. 44(a)]. Besides the practical importance of fast DW motion, the physical parameter determining this improvement was the structural inversion asymmetry (Miron et al., 2009). Indeed, while $\mathrm{Pt} / \mathrm{Co} / \mathrm{AlOx}$ supports fast $\mathrm{CIDM}$, magnetically similar $\mathrm{Pt} / \mathrm{Co} / \mathrm{Pt}$ symmetric layers do not exhibit any CIDM at all (Cormier et al., 2010; Miron et al., 2009). These first observations were initially analyzed within the framework of the STT model, including the influence of the FL SOT, which was discovered at the same time. The broken symmetry could accelerate the spin flip rate and enhance the non-adiabatic torque, while the FL SOT stabilizes the Bloch wall (BW) structure to prevent the Walker breakdown (Miron et al., 2011b).

At that stage, there was still a major discrepancy between the STT model and the experiment: the DWs move in the direction of the electric current and not along (a)

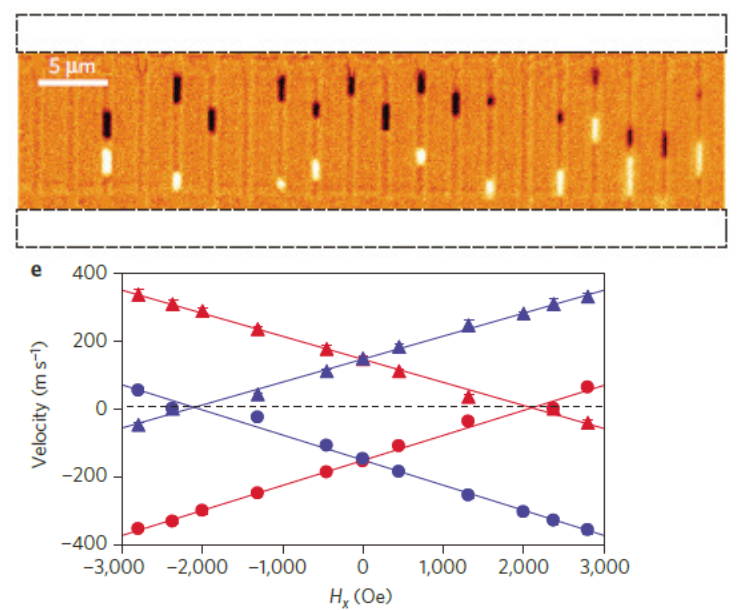

FIG. 44 (a) Differential Kerr microscopy imaging of DW displacements (stripes of black or white contrast) in an array of $\mathrm{Pt} / \mathrm{Co} 0.6 \mathrm{~nm} / \mathrm{AlOx} 500 \mathrm{~nm}$ wide nanostrips, after 20 current pulses $\left(J=1.2 \times 10^{12} \mathrm{~A} / \mathrm{m}^{2}, 3 \mathrm{~ns}\right.$ duration) (Miron et al., 2010). (b) Observation of chiral effects: the velocity of up/down and down/up DWs (blue and red) is the same, but becomes different when an in-plane field is applied [sample $\mathrm{Pt} / \mathrm{CoNiCo} / \mathrm{TaN}$, current density $J=1.5 \times 10^{12} \mathrm{~A} / \mathrm{m}^{2}$, either positive (triangles) or negative (circles)]. Within the DMI-SOT model, the DMI field strength is indicated by the value of the crossing field, where the DW velocity changes sign (Ryu et al., 2013).

that of the electron flow (Moore et al., 2009). This intriguing observation motivated several theoretical studies, which found that the combination of STT and SOT could in certain cases produce backwards motion (Boulle et al., 2014; Kim et al., 2012b). However these scenarii were not robust: the backward motion was only obtained for certain values of the physical parameters and only in a certain range of current density. In parallel, it was observed that nearly symmetric $\mathrm{Pt} / \mathrm{Co} / \mathrm{Pt}$ samples exhibit CIDM if an external in-plane field is applied parallel to the current, sufficiently large to convert BWs to NWs (Haazen et al., 2013) [the NW structure under inplane field was later confirmed by anisotropic magnetoresistance measurements (Franken et al., 2014)]. The DL SOT mechanism was shown to be compatible with all observations, especially (i) the reversal of the DW motion upon locating the thicker Pt layer below or above the Co layer; (ii) the reversal of the DW motion upon change of sign of the in-plane field and (iii) the fact that two successive DWs always move in opposite directions. The latter point is of crucial importance: all NWs, having the same magnetization, feel the same DL SOT and are hence displaced in opposite directions, like under an easy-axis ( $z$ here) field.

In this context, a breakthrough was the micromagnetic study (Thiaville et al., 2012) of the dynamics of NWs under magnetic field and DL SOT, in the case where such walls are stabilized by the interfacial DMI. 
The DMI (Dzyaloshinskii, 1957; Moriya, 1960) is an antisymmetric exchange interaction that is allowed when the medium does not have inversion symmetry. In an isotropic bulk material without inversion symmetry (like a heap of screws), to the lowest order in gradient expansion, DMI in continuous micromagnetic form reads (Bogdanov and Yablonskii, 1989)

$$
W_{3 \mathrm{D}}=D_{3 \mathrm{D}} \mathbf{m} \cdot(\boldsymbol{\nabla} \times \mathbf{m}) .
$$

Such an interaction favors helicoidal magnetization rotations of a given handedness. Referring to Fig. 43, this form of DMI stabilizes chiral BWs or Y-BWs.

On the other hand, at the interface between two dissimilar materials where inversion symmetry is structurally broken (Fert, 1990), assuming the highest symmetry $\left(C_{\infty v}\right)$ and considering the lowest order in spatial gradient, one obtains(Bogdanov and Yablonskii, 1989; Heide et al., 2008)

$$
W_{2 \mathrm{D}}=D_{2 \mathrm{D}} \mathbf{m} \cdot[(\mathbf{z} \times \boldsymbol{\nabla}) \times \mathbf{m}] .
$$

This interaction, called interfacial DMI, favors cycloidal magnetization rotations of a given handedness. Again referring to Fig. 43, this form of DMI stabilizes chiral NWs (but none of the Y-NWs). The immediate consequence is that chiral NWs move under DL SOT without any in-plane field, with successive walls moving in the same direction as their DW magnetizations are opposite. Such a motion, already obtained with STT, is required for DW racetrack applications (Parkin et al., 2008). Another notable feature of the DW dynamics under DMI and DL SOT is that the relative sign of DW velocity with respect to that of the current is given by the product of the sign of the DL SOT and the sign of the DMI.

Interfacial DMI was already evidenced in magnetic atomic monolayers or bilayers by spin-polarized scanning tunneling microscopy that revealed magnetization cycloids of fixed handedness (Bode et al., 2007; Meckler et al., 2009). However, these were situations of very large DMI so that the uniform magnetic state was destabilized. For the $\mathrm{Pt} / \mathrm{Co} / \mathrm{AlOx}$ case, direct proof that $\mathrm{DWs}$ are chiral NWs was obtained by NV-center magnetic microscopy (Tetienne et al., 2015), and by x-ray magnetic circular dichroism (Boulle et al., 2016). In addition, spinpolarized low energy electron microscopy has shown the change of DW structure from chiral NW to achiral BW as a function of the thickness of the magnetic layer (Chen et al., 2013a,b), confirming the interfacial DMI description.

The prediction of Thiaville et al. (2012) was immediately backed by two experimental papers (Emori et al., 2013; Ryu et al., 2013). As the sign of the SHE (hence of the DL SOT) was known from other measurements, the direction of DW motion under current could be related to the sign of DMI [Fig. 44(b)]. This sign was later obtained by several other techniques, so that presently estimates of interfacial DMI for a fair number of NM/FM interfaces exist. In this picture, the $\mathrm{Pt} / \mathrm{Co}$ interface stands out with one of the largest interfacial DMI constant $D_{\mathrm{s}} \approx-1.7 \mathrm{pJ} / \mathrm{m}$ (Belmeguenai et al., 2015). One of the techniques for determining the DMI consists in applying an additional in-plane field in order to compensate the DMI effective field on the DW. At this compensation, the DW velocity crosses zero (Emori et al., 2013) [for an example see Fig. 44(b)].

\section{Domain wall motion under spin-orbit torque}

We now describe in more detail the dynamics of DWs under SOTs and DMI. Once the torques are known and quantified, the study of their impact on DW motion should ultimately be performed by numerical micromagnetic simulations, for the sample parameters and geometrical dimensions. For the physical understanding, however, simplified and as analytical as possible models are helpful. The simplest model was exposed in Subsection VI.A. The next level of complexity is addressed by the so-called $q-\Phi$ model, that describes a $1 \mathrm{D} \mathrm{DW}$ dynamics for an assumed DW profile described by only two variables, namely the DW position $q$ and the angle $\Phi$ of the DW magnetization within the plane orthogonal to the easy axis (Schryer and Walker, 1974; Slonczewski, 1972). For SOT-driven DW motion assisted by DMI, the model was established in Ref. (Thiaville et al., 2012), and further developed to incorporate in-plane fields (Emori et al., 2013) and STT (Torrejon et al., 2014). At a higher level of complexity, a numerical micromagnetic calculation is performed assuming a 1D structure and dynamics, i.e., the magnetization depends only on the $x$ coordinate, the magnetostatic effects being computed for the nanostrip width $w$ and thickness $h$. Finally, for ultrathin films the full model consists of 2D numerical micromagnetics.

Figure 45(a) shows the predicted velocity versus current curves, $v(J)$, in the case of pure DL SOT and for various values of the effective DMI energy density, $D=D_{s} / h$. The DW velocity initially rises linearly with current, following a slope that does not depend on DMI and is given, for DMI dominating the magnetostatic energy associated to a NW and using the notation of Eq. (1), by

$$
v=-\gamma \frac{\pi \Delta_{\mathrm{W}}}{2 \alpha M_{\mathrm{s}}} \tau_{\mathrm{DL}}
$$

Here, $\Delta_{\mathrm{W}}$ is the micromagnetic DW width parameter. Upon further increase of the current density, the velocity saturates towards a plateau determined by the DMI strength, $v_{D}=\gamma \pi D /\left(2 M_{\mathrm{s}}\right)$ (derived in the same limit). The velocity saturation is physically explained by the progressive rotation of the DW magnetization from Néel to Bloch around the effective field $\mathbf{B}$ associated with the DL SOT. This rotation leads to a reduction of the DL SOT on the DW, as the torque vanishes for a $\mathrm{BW}$. This 
(a)
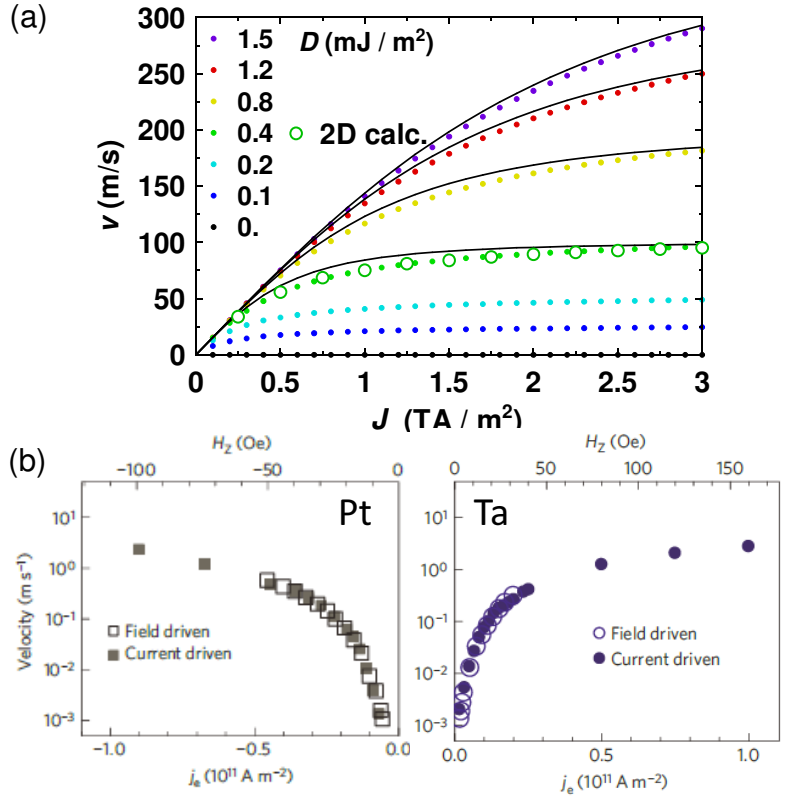

FIG. 45 Velocity of DWs in ultrathin Pt/Co/oxide films with DMI, under current. (a) Micromagnetic 1D calculations (points) of DW velocity versus current density, for various values of effective DMI in a $0.6 \mathrm{~nm}$ Co film (Thiaville et al., 2012), considering only DL SOT. Curves show the $q-\Phi$ model results for comparison. (b) Measured DW velocity under current for $\mathrm{Pt} / \mathrm{CoFe} / \mathrm{MgO}$ and $\mathrm{Ta} / \mathrm{CoFe} / \mathrm{MgO}$ (Emori et al., 2013). The CoFe film has $80-20$ atomic composition ans is 0.6 $\mathrm{nm}$ thick. Note the log scale for velocities, and the opposite current signs for the two heavy metal layers.

behavior is in good overall agreement with experiments, see e.g. Fig. 45(b). With intrinsic curvature and no Walker breakdown, the velocity versus current behavior, $v(J)$, is markedly different from that expected for STT.

When DMI is not much larger than the magnetostatic energy associated to the NW, the situation is more complex to analyze, as the velocity $v_{D}$ decreases and becomes comparable to that induced by STT. Moreover, for the $q-\Phi$ model, the analytical expressions become much more complex. The analysis of the DMI versus DW magnetostatics competition, together with that of DL SOT versus STT, was performed by (Torrejon et al., 2014) in the case of $\mathrm{HM} / \mathrm{CoFeB} / \mathrm{MgO}$ for $\mathrm{HM}=\mathrm{Hf}, \mathrm{Ta}, \mathrm{TaN}, \mathrm{W}$ i.e., the beginning of the $5 d$ series, using the $q-\Phi$ model to analyze the experiments. This showed that the determination of the DMI by the 'crossing field' technique is strongly affected by the STT when DMI is not large.

\section{Two-dimensional effects in current-induced domain wall motion}

Unlike in-plane magnetized nanowires, where DWs behave as quasi-1D objects, in perpendicular samples DWs act more like 2D membranes. One of the first observa- tions on the influence of the 2D character on the CIDM in materials with broken inversion symmetry was the occurrence of a DW tilt. When DWs are displaced by sufficiently long current pulses, their end position is no longer perpendicular to the wire (at the energy minimum), but tilted at a certain angle (Ryu et al., 2012) [see Fig. 46(a)]. Boulle et al. (2013) proposed that this tilting arises from the competition between the DL SOT and the DMI. Because the DMI energy prefers that the DW magnetization is perpendicular to the DW, the DL SOT acting on the DW magnetization modifies the DW angle [see also Martinez et al. (2014)]. A direct consequence of this current-driven tilting is an additional deformation of the $v(J)$ curve at large current density. For DL SOT only, this gives rise to a velocity increase close to the threshold for domain stability given by $\tau_{\mathrm{DL} \max }=\gamma B_{K \text { eff }} / 2$ ( $B_{K \text { eff }}$ being the effective perpendicular anisotropy that incorporates the demagnetizing field). a

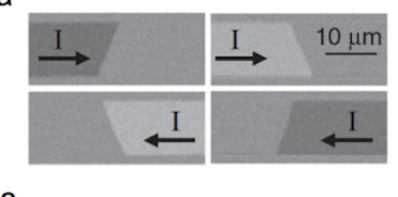

C
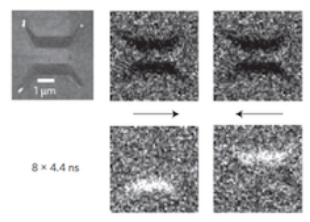
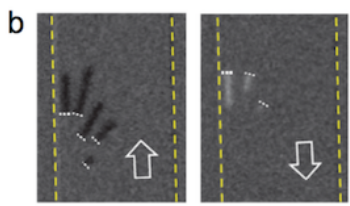

凤
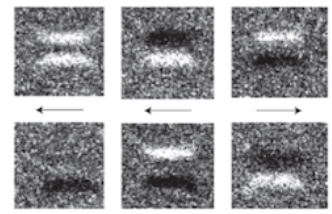

FIG. 46 (a) Kerr imaging of DW tilting produced by current injection (Ryu et al., 2012). (b) Kerr images of current induced DW motion in a non-collinear geometry. (c) Magnetization reversal controlled by geometry (Safeer et al., 2016).

It was recently shown that the $2 \mathrm{D}$ character of CIDM can be exploited for DW manipulation. Since the DW magnetization is either aligned (BW) or perpendicular (NW) to the DW direction, the control of the DW tilt allows for setting the SOT efficiency by modifying the angle between the electric current and the magnetization of the DW. Using this approach, Safeer et al. (Safeer et al., 2016) have shown that the current induced DW motion in the non-collinear geometry exhibits surprising features [see Fig. 46(b)]. Namely, depending on their polarity (up/down or down/up), the DWs move faster for a certain sign of the electric current. This phenomenon links the polarity of the DWs with their direction of motion. Therefore, by controlling the shape of a magnetic layer, one can control its magnetization reversal [Fig. 46(c)]. 
4. Domain wall motion under combined spin transfer and spin-orbit torques

A detailed study of CIDM in $\mathrm{Pt} /(\mathrm{Co} / \mathrm{Ni})_{N} / \mathrm{Co} / \mathrm{MgO}$ as a function of magnetic layer thickness (by varying the repetition number $N$ ) was realized by Ueda et al. (2014). The interest in the $(\mathrm{Co} / \mathrm{Ni})_{N}$ multilayer system is driven by the property that, since magnetic anisotropy arises from the internal $\mathrm{Co} / \mathrm{Ni}$ interfaces, the total thickness of the multilayer can be changed while keeping the same magnetic anisotropy, which is not possible for a single Co layer. The DWs were observed to move along the electrons for large thicknesses $(N>6)$, but in the opposite direction at small thicknesses $(N<3)$. From the application of additional easy-axis field, it was concluded that CIDM at large thickness is due to the adiabatic STT, but that the torque on the DW is like a bias field for low thickness. Applying then, in addition to current, in-plane fields in both orientations (longitudinal $x$, and transverse $y$ ), the crossing field effect [see Fig. 44(b)] was observed in the longitudinal case, in accord with the DL SOT in the presence of DMI. The transverse field was observed to linearly modify the velocity of both up/down and down/up DWs, in the same way. This is also consistent with the DMI and DL SOT mechanism, as the magnetizations of two consecutive chiral NWs precess under the respective fields $\mathbf{B}$ of the DL SOT towards the same $y$ direction. Thus, for not too large $y$ fields, one polarity will increase this rotation and hence decrease the DW velocities, whereas the other polarity will decrease this rotation and increase the velocities. From the symmetry of the effects, the authors concluded that the FL SOT effect was negligible. Direct measurements of the two components of the SOT confirmed the reduced value of the FL SOT. This work clearly evidences the transition from bulk to interfacial CIDM and can serve as a guide for further studies of this physics. For example, the absence of DW motion for $3 \leq N \leq 6$ was interpreted by the fact that the interfacial DMI from the bottom $\mathrm{Pt}$ layer was raising too much the Walker field, so that the DW motion by adiabatic STT could not be reached for the applied currents. The same mechanism applies for the combination of adiabatic STT and FL SOT, showing that these two mechanisms of CIDM can act in opposition. A similar transition from SOT to STT driven DW motion has been observed in $(\mathrm{Co} / \mathrm{Tb})_{N}$ multilayers (Bang et al., 2016).

In another study in the same $(\mathrm{Co} / \mathrm{Ni})_{N}$ system, the structure was designed such that SOT acted as a perturbation with respect to STT (Yoshimura and Koyama, 2014). The sample was medium-thick $(N=4)$ and the structure was nominally symmetric with $\mathrm{Pt}$ and $\mathrm{Ta}$ on both sides, with the same thicknesses. The DW motion, driven by STT, was modified by applying in-plane fields, both along the current $(x)$ or transverse $(y)$. As expected for adiabatic STT, the motion was suppressed by large in-plane fields, as these fields block the precession of the DW moment. The surprise was that the DW motion windows were not centered at zero field, with the $x$-field offset reversing sign between up-down and downup DWs. This could be qualitatively interpreted by (i) a precession dissymmetry under in-plane field that leads to different residence times for NWs of opposite chiralities, and (ii) a non-compensated DL SOT due to a measured imbalance in the conduction of the top and bottom Pt layers. On the other hand, the independence of the $y$ field offset on the DW type (up/down or down/up) is consistent with an effect of Oersted field and/or FL SOT This work, more generally, proposes a way to experimentally test the presence of the SOT and of the Oersted field, as any in-plane field affects the precession of the DW moment triggered by STT. Here we refer also to the numerical work by (Martinez, 2012) on the STT plus FL SOT case, for various values on non-adiabaticity, and the micromagnetic simulations analysis by (Boulle et al., 2014; Martinez et al., 2013) of experimental results for $\mathrm{Pt} / \mathrm{Co} / \mathrm{AlOx}$ in terms of STT plus SOT.

\section{Motion of magnetic skyrmions under spin-orbit torques}

Magnetic skyrmions with non-zero spin winding number are compact magnetic textures with a non-trivial topology, so that they cannot be removed by a continuous perturbation. Note that there are still arguments about the precise signification of this terminology; we will stick here to the definition agreed on by a large panel of authors (Hellman et al., 2017). There is currently an increasing interest in the electrical manipulation of such objects as they could serve as fundamental building blocks for data storage and logic devices (Fert et al., 2013; Tomasello et al., 2014; Zhang et al., 2015d). Skyrmions have, in addition to topology and compared to the magnetic bubbles extensively investigated in the past (Malozemoff and Slonczewski, 1979), a fixed chirality which is an important asset for SOT as can be inferred from the preceding considerations.

A physically appealing way to understand how the various characteristics of a skyrmion affect its response to current-induced torques is offered by Thiele's equation derived from the LLG equations to handle the steadystate motion of rigid textures (Thiele, 1973). Thiele's equation has been generalized to include STT (Thiaville et al., 2005) and, more recently, also SOT (Sampaio et al., 2013). It reads,

$$
\mathbf{G} \times(\mathbf{v}-\mathbf{u})-\overline{\bar{D}}(\alpha \mathbf{v}-\beta \mathbf{u})+\mathbf{F}_{\mathrm{SOT}}+\mathbf{F}=\mathbf{0},
$$

where $\mathbf{v}$ is the in-plane velocity of the skyrmion center, $\mathbf{u}$ is the spin-drift velocity, $\beta$ is the non-adiabaticity parameter related to STT [see Eq. (64)], G is the so-called gyrovector, $\overline{\bar{D}}$ is the dissipation tensor introduced by Thiele 
(a)

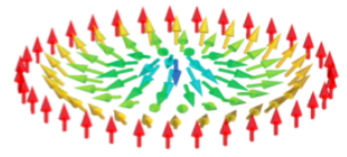

(c)
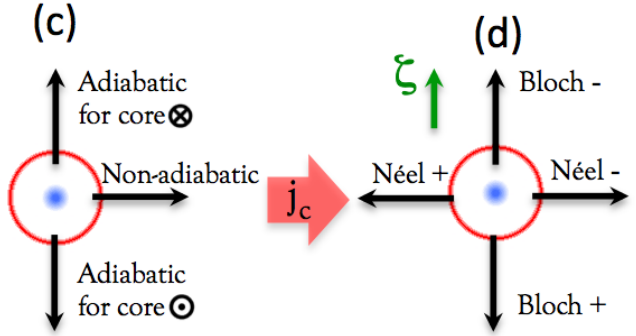

FIG. 47 (a) Néel- and (b) Bloch-skyrmions (both with negative polarity), adapted from (Fert et al., 2017). (c) Schematic of the forces (black arrow) applied to a skyrmion (circle) by a current density $j_{\mathrm{c}}$, in the case of adiabatic and non-adiabatic STT. The forces are independent on the type of the skyrmion and only depend on its core polarity. (d) Schematic of the forces (black arrows) applied to a skyrmion (circle) by a DL SOT with a spin polarization along $\boldsymbol{\zeta}$, as indicated by the green arrow. The forces depend on whether the skyrmion is Bloch or Néel.

(from which the damping coefficient $\alpha$ was factored out when generalizing to STT), $\mathbf{F}$ is the other force applied to the skyrmion (e.g., pinning), and finally $\mathbf{F}_{\mathrm{SOT}}$ is the force that SOT applies to the skyrmion. Topology appears in the gyrovector $\mathbf{G}=\left(M_{\mathrm{s}} h / \gamma\right) 4 \pi N_{\mathrm{Sk}} \mathbf{z}$, that is along the film normal and proportional to the topological (or skyrmion) number $N_{\mathrm{Sk}}$. The latter is simply, for a compact texture, $N_{\mathrm{Sk}}=S p$ with $p$ the polarity of the magnetization of the skyrmion center $(+1$ for $+\mathbf{z})$ and $S$ the winding number of the magnetization $(+1$ for the simple skyrmions). The dissipation tensor, diagonal for high-symmetry textures, is related to the size of the skyrmion [see e.g. Hrabec et al. (2017)]. The force $\mathbf{F}$ is non-zero for example when a confining potential exists, or a small $z$ field gradient. The STT forces on a skyrmion are illustrated in Fig. 47(c).

The force from the SOT is computed by projecting the SOT on the skyrmion displacement (the procedure by which Thiele's equation is constructed), as a volume integral for each component $i$,

$$
F_{i, \mathrm{SOT}}=-\int \mathbf{B}_{\mathrm{SOT}} \cdot \partial_{i} \mathbf{M}=\tau_{\mathrm{DL}} \boldsymbol{\zeta} \cdot \int \mathbf{m} \times \partial_{i} \mathbf{m} .
$$

The FL SOT gives no contribution to the force as it acts like a constant in-plane field. As for the DL SOT contribution, remembering that $\boldsymbol{\zeta} \| \mathbf{y}$ for current along $x$, Eq. (69) amounts to one term of the DMI energy density. For the $x$ component of the force (along the current), it is the part of the interfacial DMI that involves the $x$ gradients [Eq. (66)]. For the $y$ component of the force (transverse to the current), it is the part of the bulk DMI that involves the $y$ gradients [Eq. (65)]. Thus, the DL SOT force on a skyrmion depends on its chirality and of its type (i.e. Bloch or Néel), see Fig. 47(d). Because of the gyrotropic term, both STTs and DL SOT will drive the skyrmion, at some angle between the $x$ and $y$ axes, so that skyrmion motion under current alone does not allow to infer its internal structure. Nevertheless, conventional magnetic bubbles, whose lowest energy state is an achiral Bloch skyrmion because of the absence of significant DMI, would be sorted according to their Bloch chirality by DL SOT. This is in contrast to skyrmions that have a definite chirality (fixed by DMI) and should all follow the same trajectory.

Metastable magnetic skyrmions have been recently obtained at room temperature in transition metal multilayers (Boulle et al., 2016; Chen et al., 2015; Hrabec et al., 2017; Jiang et al., 2015; Moreau-Luchaire et al., 2016; Pollard et al., 2017; Woo et al., 2016). Many experiments have revealed skyrmions motion under current, either along or against the direction of the electron flow, in most cases in agreement with the DMI and DL SOT sign (Hrabec et al., 2017; Jiang et al., 2015, 2017b; Litzius et al., 2017; Woo et al., 2016; Yu et al., 2016a). A prediction of Thiele's equation, namely the value of the angle of the gyrotropic deflection (sometimes also called skyrmion Hall angle), is however poorly reproduced experimentally in most cases. This may be due to disorder (Jiang et al., 2017b; Kim and Yoo, 2017; Legrand et al., 2017). Another effect, beyond the rigid skyrmion model, is that the skyrmion dynamic deformation leads to an influence of the FL SOT on the deflection angle (Litzius et al., 2017). Finally, the influence of the gradient of the $z$-component of the Oersted field should also be checked (Hrabec et al., 2017). Altogether, skyrmions appear as favorable objects to be controlled by either STT or SOT since their velocity reach that of magnetic DWs in the same structures. Further exploration of their robustness and scalability is currently on-going.

\section{Domain wall motion in disordered media}

In sputtered ultrathin magnetic multilayers, disorder is so strong that CIDM only occurs at large (field or current) drive. At low drive, DW motion is not described by LLG equations but consists of thermally assisted hopping between pinning sites. This regime of DW motion is called creep, or depinning (Gorchon et al., 2014; Metaxas et al., 2007). Whereas field-driven creep seemed to be well understood, the situation has changed with the introduction of STT, SOT and DMI. Several experiments of CIDM have shown that the creep regime of DW motion deserves further study in order to be fully understood (Lavrijsen et al., 2012, 2015; Vanatka et al., 2015). For 
instance, it was recently proposed that structural inversion asymmetry could be responsible for a chiral dissipation mechanism affecting the DW dynamics (Akosa et al., 2016; Jué et al., 2016). More recently it has been shown that, as the DW energy becomes orientation-dependent under in-plane field (Pellegren et al., 2017) - an effect reinforced by DMI and strikingly evidenced by specific domain shapes (Lau et al., 2016a) - the simple creep model with uniform DW tension fails. Thus, the analysis of creep motion under in-plane field has to be thoroughly re-examined.

\section{Antiferromagnetic and ferrimagnetic systems}

The search for extremely high DW velocity has recently brought perpendicularly magnetized synthetic AF strips, such as $(\mathrm{Co} / \mathrm{Ni}) / \mathrm{Ru} /(\mathrm{Co} / \mathrm{Ni})$, to the forefront. In such systems, Yang et al. (2015) reported SOT driven DW velocities as fast as $750 \mathrm{~m} / \mathrm{s}$ and explained the results by the enhanced Walker breakdown threshold. Indeed, in the presence of Ru-mediated interlayer exchange coupling (RKKY) the azimuthal angles of the two antiparallel DWs stabilize each other such that the DW propagates in the flow regime over a larger range of driving current densities [see also (Lepadatu et al., 2017)]. In a recent work Qiu et al. (2016) observed that the presence of the $\mathrm{Ru}$ spacer layer may affect the spin current, leading to different SOTs for such trilayers.

Similar ideas resulted in the proposition of $\mathrm{AF}$ skyrmions, either in the bilayer form or in bulk AFs, which display no skyrmion Hall effect and could also reach very high velocity in the latter case (Barker and Tretiakov, 2016; Tomasello et al., 2017; Zhang et al., 2015e). Bulk AFs are particularly interesting for their ability to support $\mathrm{THz}$ dynamics, and it was recently proposed that AF DWs driven by SOT could reach extremely high velocities, while displaying a Lorentz contraction when reaching the spin wave group velocity (Gomonay et al., 2016; Shiino et al., 2016). The investigation of $\mathrm{AF}$ spintronics is still at its infancy though (Jungwirth et al., 2016; V. Baltz, A. Manchon, M. Tsoi, T. Moriyama, T. Ono, 2017), and alternative materials are being explored. From this perspective, ferrimagnets such as FeGdCo offer an appealing platform due to the tunability of their compensation point. For instance, recent studies have demonstrated large enhancement of field-driven DW velocity and SOT efficiencies close to the angular momentum compensation point (Kim et al., 2017a; Mishra et al., 2017).

\section{PERSPECTIVES}

The interconnection between spin and orbital angular momenta in materials lacking inversion symmetry has led to the theoretical prediction and experimental observation of a wealth of fascinating phenomena, ranging from SOTs and charge pumping to chiral DWs and magnetic skyrmions. These effects are usually accompanied by a complex form of the conductivity tensor and unusual dynamical behavior that render the experimental identification of the physics at stake quite challenging. Nonetheless, SOTs are now observed systematically in a wide range of materials, and enable very efficient ways to control the magnetic order in various configurations. The application of these concepts to working devices, such as SOT-MRAM, nano-oscillators or magnetic racetrack data storage devices, remains an active area of research.

The recent extension of these effects to AFs (Wadley et al., 2016) and magnetic insulators (Avci et al., 2017) opens particularly stimulating perspectives as these classes of materials bear specific dynamical features $(\mathrm{THz}$ excitations for the former, long range spin propagation phenomena for the latter) that are unknown in traditional FMs. Along this line, one could extend these ideas to frustrated magnets that support exotic magnetic behaviors (Balents, 2010).

Finally, while transition metals $(\mathrm{Pt}, \mathrm{W} \ldots)$ have been the center of attention in the early development of this field, the recent outburst of topological materials (Hasan and Kane, 2010; Qi and Zhang, 2011; Vafek and Vishwanath, 2014; Wehling et al., 2014) and two dimensional lattices (Castro Neto et al., 2009; Xu et al., 2014) opens novel directions. The ever-expanding zoology (topological insulators, Dirac semimetals, Weyl semimetals, Kondo topological insulators etc.) and continuous synthesis of such materials (bismuth chalcogenides, graphene and its siblings, transition metal dichalogenides, $\mathrm{MPX}_{3} \ldots$ ) offer a unique opportunity for the discovery of efficient and exotic spin-charge conversion mechanisms and chiral spin textures.

\section{LIST OF ABBREVIATIONS}

1D, 2D, 3D: One-, two- and three-dimensional AF: Antiferromagnet

AHE: Anomalous Hall effect

AMR: Anisotropic magnetoresistance

ANE: Anomalous Nernst effect

BW: Bloch wall

CIDM: Current-induced domain wall motion

DFT: Density functional theory

DMI: Dzyaloshinskii-Moriya interaction

DL: Damping-like (torque)

DW: Domain wall

FL: Field-like (torque)

FM: Ferromagnet

FMR: Ferromagnetic resonance

HHV: Harmonic Hall voltage analysis

iSGE: Inverse spin galvanic effect 
LED: Light emitting diode

LLG: Landau-Lifshitz-Gilbert (equation)

MOKE: Magneto-optical Kerr effect

MRAM Magnetic random access memory

NM: Non-magnetic metal

NW: Néel wall

RF: Radio frequency

RKKY: Ruderman-Kittel-Kasuya-Yosida (interaction)

SGE: Spin galvanic effect

SHE: Spin Hall effect

SOT: Spin-orbit torque

ST-FMR: Spin torque ferromagnetic resonance

STT: Spin transfer torque

TMR: Tunnelling magnetoresistance

TW: Transverse wall

VW: Vortex wall

\section{ACKNOWLEDGEMENTS}

A.M. was supported by the King Abdullah University of Science and Technology (KAUST). T. J. acknowledges support from the EU FET Open RIA Grant No. 766566, the Ministry of Education of the Czech Republic Grant No. LM2015087 and LNSM-LNSpin, and the Grant Agency of the Czech Republic Grant No. 14-37427G. J. S. acknowledges the Alexander von Humboldt Foundation, EU FET Open Grant No. 766566, EU ERC Synergy Grant No. 610115, and the Transregional Collaborative Research Center (SFB/TRR) $173 \mathrm{SPIN+X.} \mathrm{K.G.}$ and P.G. acknowledge stimulating discussions with C.O. Avci and financial support by the Swiss National Science Foundation (Grant No. 200021-153404) and the European Commission under the Seventh Framework Program (spOt project, Grant No. 318144).

\section{REFERENCES}

Akosa, C A, I. M. Miron, G. Gaudin, and A. Manchon (2016), "Phenomenology of chiral damping in noncentrosymmetric magnets," Physical Review B 93, 214429.

Akyol, Mustafa, Wanjun Jiang, Guoqiang Yu, Yabin Fan, Mustafa Gunes, Ahmet Ekicibil, Pedram Khalili Amiri, and Kang L. Wang (2016), "Effect of heavy metal layer thickness on spin-orbit torque and current-induced switching in $\mathrm{Hf} / \mathrm{CoFeB} / \mathrm{MgO}$ structures," Applied Physics Letters 109 (2), 022403.

Allen, Gary, Sasikanth Manipatruni, Dmitri E. Nikonov, Mark Doczy, and Ian A. Young (2015), "Experimental demonstration of the coexistence of spin Hall and Rashba effects in $\beta$-tantalum/ferromagnet bilayers," Physical Review B 91, 144412.

Allwood, D A, G. Xiong, C. C. Faulkner, D. Atkinson, D. Petit, and R. P. Cowburn (2005), "Magnetic Domain-Wall Logic," Science 309, 1688.

Amin, V P, and M. D. Stiles (2016a), "Spin transport at interfaces with spin-orbit coupling: Formalism," Physical Review B 94, 104419.
Amin, V P, and M. D. Stiles (2016b), "Spin transport at interfaces with spin-orbit coupling: Phenomenology," Physical Review B 94, 104420.

An, Hongyu, Yuito Kageyama, Yusuke Kanno, Nagisa Enishi, and Kazuya Ando (2016), "Spin-torque generator engineered by natural oxidation of $\mathrm{Cu}$," Nature Communications 7, 13069.

An, Kyongmo, Daniel R. Birt, Chi Feng Pai, Kevin Olsson, Daniel C. Ralph, R. A. Buhrman, and Xiaoqin Li (2014), "Control of propagating spin waves via spin transfer torque in a metallic bilayer waveguide," Physical Review B 89, 140405(R).

Ando, K, S. Takahashi, K. Harii, K. Sasage, J. Ieda, S. Maekawa, and E. Saitoh (2008), "Electric Manipulation of Spin Relaxation Using the Spin Hall Effect," Physical Review Letters 101 (3), 036601.

Ando, Kazuya (2014), "Dynamical generation of spin currents," Semiconductor Science and Technology 29 (4), 043002.

Aradhya, S V, G. E. Rowlands, J. Oh, D. C. Ralph, and R. A. Buhrman (2016), "Nanosecond-Timescale Low Energy Switching of In-Plane Magnetic Tunnel Junctions through Dynamic Oersted-Field-Assisted Spin Hall Effect," Nano Letters 16 (10), 5987-5992.

Aronov, A G, and Y. B. Lyanda-Geller (1989), "Nuclear electric resonance and orientation of carrier spins by an electric field," JETP Lett. 50, 398.

Avci, Can Onur, Kevin Garello, Mihai Gabureac, Abhijit Ghosh, Andreas Fuhrer, Santos F Alvarado, and Pietro Gambardella (2014a), "Interplay of spin-orbit torque and thermoelectric effects in ferromagnet/normal-metal bilayers," Physical Review B 90, 224427.

Avci, Can Onur, Kevin Garello, Abhijit Ghosh, Mihai Gabureac, Santos F Alvarado, and Pietro Gambardella (2015a), "Unidirectional spin Hall magnetoresistance in ferromagnet/normal metal bilayers," Nature Physics 11 (7), 570575 .

Avci, Can Onur, Kevin Garello, Johannes Mendil, Abhijit Ghosh, Nicolas Blasakis, Mihai Gabureac, Morgan Trassin, Manfred Fiebig, and Pietro Gambardella (2015b), "Magnetoresistance of heavy and light metal/ferromagnet bilayers," Applied Physics Letters 107 (19), 192405.

Avci, Can Onur, Kevin Garello, Ioan Mihai Miron, Gilles Gaudin, S. Auffret, Olivier Boulle, and Pietro Gambardella (2012), "Magnetization switching of an $\mathrm{MgO} / \mathrm{Co} / \mathrm{Pt}$ layer by in-plane current injection," Applied Physics Letters 100 (21), 212404.

Avci, Can Onur, Kevin Garello, Corneliu Nistor, Sylvie Godey, Belén Ballesteros, Aitor Mugarza, Alessandro Barla, Manuel Valvidares, Eric Pellegrin, Abhijit Ghosh, Ioan Mihai Miron, Olivier Boulle, S. Auffret, Gilles Gaudin, and Pietro Gambardella (2014b), "Fieldlike and antidamping spin-orbit torques in as-grown and annealed Ta/CoFeB/MgO layers," Physical Review B 89, 214419.

Avci, Can Onur, Andy Quindeau, Chi-Feng Pai, Maxwell Mann, Lucas Caretta, Astera S Tang, Mehmet C Onbasli, Caroline A Ross, and Geoffrey S D Beach (2017), "Currentinduced switching in a magnetic insulator," Nature Materials 16 (November), 309.

Bader, S D, and S.S.P. Parkin (2010), "Spintronics," Annual Review of Condensed Matter Physics 1 (1), 71-88.

Baek, Seung-heon Chris, Vivek P. Amin, Young-Wan Oh, Gyungchoon Go, Seung-Jae Lee, M. D. Stiles, Byong-Guk Park, and Kyung-Jin Lee (2017), "Spin-orbit torques in- 
duced by interface-generated spin currents," arXiv preprint arXiv:1708.06864 .

Balents, Leon (2010), "Spin liquids in frustrated magnets." Nature 464, 199-208.

Bang, Do, Jiawei Yu, Xuepeng Qiu, Yi Wang, Hiroyuki Awano, Aurelien Manchon, and Hyunsoo Yang (2016), "Enhancement of spin Hall effect induced torques for current-driven magnetic domain wall motion: Inner interface effect," Physical Review B 93 (17), 174424.

Barker, Joseph, and Oleg A. Tretiakov (2016), "Static and Dynamical Properties of Antiferromagnetic Skyrmions in the Presence of Applied Current and Temperature," Physical Review Letters 116, 147203.

Bauer, Uwe;, Yao; Lide, and Geoffrey S. D. Beach (2015), "Magneto-ionic control of interfacial magnetism," Nature materials 14 (0), 174-181.

Baumgartner, M (2018), Spatially and time-resolved measurements of the magnetization dynamics driven by spin-orbit torques, Phd thesis (ETH Zurich).

Baumgartner, Manuel, Kevin Garello, Johannes Mendil, Can Onur Avci, Eva Grimaldi, Christoph Murer, Junxiao Feng, Mihai Gabureac, Christian Stamm, Yves Acremann, Simone Finizio, Sebastian Wintz, Jörg Raabe, and Pietro Gambardella (2017), "Spatially and time-resolved magnetization dynamics driven by spin-orbit torques," Nature Nanotechnology 12 (10), 980-986.

Beach, G S D, M. Tsoi, and J. L. Erskine (2008), "Currentinduced domain wall motion," Journal of Magnetism and Magnetic Materials 320 (7), 1272-1281.

Bedau, D, H. Liu, J. Z. Sun, J. A. Katine, E. E. Fullerton, S. Mangin, and A. D. Kent (2010), "Spin-transfer pulse switching: From the dynamic to the thermally activated regime," Applied Physics Letters 97, 262502.

Belkov, V V, and S. D. Ganichev (2008), "Magnetogyrotropic effects in semiconductor quantum wells," Semicond. Sci. Technol. 23, 114003.

Belmeguenai, Mohamed, Jean Paul Adam, Yves Roussigne, Sylvain Eimer, Thibaut Devolder, Joo Von Kim, Salim Mourad Cherif, Andrey Stashkevich, and Andre Thiaville (2015), "Interfacial Dzyaloshinskii-Moriya interaction in perpendicularly magnetized $\mathrm{Pt} / \mathrm{Co} / \mathrm{AlOx}$ ultrathin films measured by Brillouin light spectroscopy," Physical Review B 91 (18), 180405.

Bernevig, B Andrei, and Oskar Vafek (2005), "Piezomagnetoelectric effects in p-doped semiconductors," Physical Review B 72, 033203.

Bhowmik, Debanjan, Long You, and Sayeef Salahuddin (2014), "Spin Hall effect clocking of nanomagnetic logic without a magnetic field." Nature Nanotechnology 9, 59.

van der Bijl, E, and R. a. Duine (2012), "Current-induced torques in textured Rashba ferromagnets," Phys. Rev. B 86 (9), 094406.

Blügel, Stefan, and Gustav Bihlmayer (2007), "Magnetism of Low-dimensional Systems : Theory," in Handbook of Magnetism and Advanced Magnetic Materials., Vol. 1, edited by H. Kronmuller and S. Parkin (John Wiley \& Sons) pp. $1-42$.

Bode, M, M Heide, $\mathrm{K}$ von Bergmann, $\mathrm{P}$ Ferriani, Stefan Heinze, G Bihlmayer, A Kubetzka, O Pietzsch, Stefan Blügel, and R Wiesendanger (2007), "Chiral magnetic order at surfaces driven by inversion asymmetry." Nature 447 (7141), 190-3.

Bodnar, S Yu, L. Šmejkal, I. Turek, T. Jungwirth, O. Gomonay, J. Sinova, A. A. Sapozhnik, H. J. Elmers,
M. Kläui, and M. Jourdan (2017), "Writing and Reading antiferromagnetic Mn2Au: Néel spin-orbit torques and large anisotropic magnetoresistance," arXiv preprint arXiv:1706.02482

Bogdanov, A, and D. A. Yablonskii (1989), "Thermodynamically stable vortices states in magnetically ordered crystals. The mixed state of magnets," Zh. Eksp. Teor. Fiz 68, 101.

Bonetti, Stefano, Vasil Tiberkevich, Giancarlo Consolo, Giovanni Finocchio, Pranaba Muduli, Fred Mancoff, Andrei Slavin, and Johan Åkerman (2010), "Experimental evidence of self-localized and propagating spin wave modes in obliquely magnetized current-driven nanocontacts," Physical Review Letters 105 (21), 217204.

Bose, Arnab, Duc Duong Lam, Swapnil Bhuktare, Sutapa Dutta, Hanuman Singh, Shinji Miwa, and Ashwin A. Tulapurkar (2017), "Observation of anomalous spin-torque generated by a ferromagnet," arXiv preprint arXiv:1706.07245

Boulle, O, L. D. Buda-Prejbeanu, E Jué, I. M. Miron, and G. Gaudin (2014), "Current induced domain wall dynamics in the presence of spin orbit torques," Journal of Applied Physics 115, 17D502.

Boulle, O, J. Kimling, P. Warnicke, M. Kläui, U. Rüdiger, G. Malinowski, H. J M Swagten, B. Koopmans, C. Ulysse, and G. Faini (2008), "Nonadiabatic spin transfer torque in high anisotropy magnetic nanowires with narrow domain walls," Physical Review Letters 101, 216601.

Boulle, O, S. Rohart, L. D. Buda-Prejbeanu, E. Jué, I. M. Miron, S. Pizzini, J. Vogel, G. Gaudin, and A. Thiaville (2013), "Domain Wall Tilting in the Presence of the Dzyaloshinskii-Moriya Interaction in Out-of-Plane Magnetized Magnetic Nanotracks," Physical Review Letters 111, 217203.

Boulle, Olivier, Jan Vogel, Hongxin Yang, Stefania Pizzini, Dayane de Souza Chaves, Andrea Locatelli, Tevfik Onur Mente Alessandro Sala, Liliana D. Buda-Prejbeanu, Olivier Klein, Mohamed Belmeguenai, Yves Roussigné, Andrey Stashkevich, Salim Mourad Chérif, Lucia Aballe, Michael Foerster, Mairbek Chshiev, Stéphane Auffret, Ioan Mihai Miron, and Gilles Gaudin (2016), "Room temperature chiral magnetic skyrmion in ultrathin magnetic nanostructures," Nature Nanotechnology 11, 449.

Brataas, Arne, Andrew D Kent, and Hideo Ohno (2012a), "Current-induced torques in magnetic materials." Nature Materials 11, 372-81.

Brataas, Arne, Yaroslav Tserkovnyak, G. E. W. Bauer, and Bertrand Halperin (2002), "Spin battery operated by ferromagnetic resonance," Physical Review B 66, 060404.

Brataas, Arne, Yaroslav Tserkovnyak, G. E. W. Bauer, and Paul J. Kelly (2012b), "Spin Pumping and Spin Transfer," arXiv preprint arXiv:1108.0385v3 .

van den Brink, Arno, Guus Vermijs, Aurélie Solignac, Jungwoo Koo, Jurgen T Kohlhepp, Henk J. M. Swagten, and Bert Koopmans (2016), "Field-free magnetization reversal by spin-Hall effect and exchange bias," Nature communications 7, 10854 .

Burrowes, C, A P Mihai, D Ravelosona, J.-V. Kim, C Chappert, L Vila, A Marty, Y Samson, F Garcia-Sanchez, L D Buda-Prejbeanu, I Tudosa, E E Fullerton, and J.-P. Attane (2010), "Non-adiabatic spin-torques in narrow magnetic domain walls," Nature Physics 6 (1), 17-21.

Castro Neto, A H, N. M. R. Peres, K. S. Novoselov, and a. K. Geim (2009), "The electronic properties of graphene," Reviews of Modern Physics 81 (1), 109-162. 
Chang, Po Hao, Troels Markussen, Søren Smidstrup, Kurt Stokbro, and Branislav K. Nikolić (2015), "Nonequilibrium spin texture within a thin layer below the surface of current-carrying topological insulator Bi2Se3: A firstprinciples quantum transport study," Physical Review B 92 (20), 201406.

Chappert, Claude, A. Fert, and Frédéric Nguyen Van Dau (2007), "The emergence of spin electronics in data storage." Nature Materials 6 (11), 813-23.

Chauleau, J Y, B. J. McMorran, R. Belkhou, N. Bergeard, T. O. Mente, M. Á Niño, A. Locatelli, J. Unguris, S. Rohart, J. Miltat, and A. Thiaville (2011), "Magnetization textures in NiPd nanostructures," Physical Review B 84 (9), 094416.

Chen, G, J Zhu, A Quesada, J Li, A T N Diaye, Y Huo, T P Ma, Y Chen, H Y Kwon, C Won, Z Q Qiu, A K Schmid, and Y Z Wu (2013a), "Novel Chiral Magnetic Domain Wall Structure in $\mathrm{Fe} / \mathrm{Ni} / \mathrm{Cu}(001)$ Films," Physical Review Letters 110, 177204.

Chen, Gong, Tianping Ma, Alpha T N'Diaye, Heeyoung Kwon, Changyeon Won, Yizheng Wu, and Andreas K Schmid (2013b), "Tailoring the chirality of magnetic domain walls by interface engineering," Nature Communications 4, 2671.

Chen, Gong, Arantzazu Mascaraque, Alpha T. N'Diaye, and Andreas K. Schmid (2015), "Room temperature skyrmion ground state stabilized through interlayer exchange coupling," Applied Physics Letters 106, 242404.

Chen, Hua, Qian Niu, and a. H. MacDonald (2014), "Anomalous Hall Effect Arising from Noncollinear Antiferromagnetism," Physical Review Letters 112, 017205.

Chen, Kai, and Shufeng Zhang (2015), "Spin pumping in the presence of spin-orbit coupling," Physical Review Letters 114, 126602.

Chen, Y L, J-H Chu, J G Analytis, Z K Liu, K Igarashi, H-H Kuo, X L Qi, S K Mo, R G Moore, D H Lu, M Hashimoto, T Sasagawa, S C Zhang, I R Fisher, Z Hussain, and Z X Shen (2010), "Massive Dirac fermion on the surface of a magnetically doped topological insulator." Science (New York, N.Y.) 329 (5992), 659-62.

Cheng, Ran, Jiang Xiao, Qian Niu, and Arne Brataas (2014), "Spin pumping and spin-transfer torques in antiferromagnets," Physical Review Letters 113, 057601.

Chernyshov, Alexandr, Mason Overby, Xinyu Liu, Jacek K. Furdyna, Yuli Lyanda-Geller, and Leonid P. Rokhinson (2009), "Evidence for reversible control of magnetization in a ferromagnetic material by means of spinorbit magnetic field," Nature Physics 5 (9), 656-659.

Chumak, A V, V. I. Vasyuchka, A. A. Serga, and Burkard Hillebrands (2015), "Magnon spintronics," Nature Physics 11 (June), 453.

Ciccarelli, C, L. Anderson, V. Tshitoyan, A. J. Ferguson, F. Gerhard, C. Gould, L. W. Molenkamp, J. Gayles, J. Zelezny, L. Smejkal, Z. Yuan, J. Sinova, F. Freimuth, and T. Jungwirth (2016), "Room-temperature spin-orbit torque in NiMnSb," Nature Physics 12 (October), 855.

Ciccarelli, Chiara, Kjetil M D Hals, A.C. Irvine, Vit Novak, Yaroslav Tserkovnyak, Hidekazu Kurebayashi, Arne Brataas, and Andrew Ferguson (2014), "Magnonic charge pumping via spin orbit coupling," Nature Nanotechnology 10, 50-54.

Clarke, D, O. Tretiakov, G.-W. Chern, Ya. Bazaliy, and O. Tchernyshyov (2008), "Dynamics of a vortex domain wall in a magnetic nanostrip: Application of the collective- coordinate approach," Physical Review B 78, 134412.

Collet, M, X de Milly, O d'Allivy Kelly, V V Naletov, R Bernard, P Bortolotti, J Ben Youssef, V E Demidov, S O Demokritov, J L Prieto, M Muñoz, V Cros, A Anane, G de Loubens, and O Klein (2016), "Generation of coherent spin-wave modes in yttrium iron garnet microdiscs by spin-orbit torque." Nature Communications 7, 10377.

Cormier, M, A. Mougin, J. Ferré, A. Thiaville, N. Charpentier, F. Piéchon, R. Weil, V. Baltz, and B. Rodmacq (2010), "Effect of electrical current pulses on domain walls in $\mathrm{Pt} / \mathrm{Co} / \mathrm{Pt}$ nanotracks with out-of-plane anisotropy: Spin transfer torque versus Joule heating," Physical Review B 81, 024407.

Cowburn, R P, and M. E. Welland (2000), "Room Temperature Magnetic Quantum Cellular Automata," Science 287 (5457), 1466-1468.

Cubukcu, Murat, Olivier Boulle, Marc Drouard, Kevin Garello, Can Onur Avci, Ioan Mihai Miron, Juergen Langer, Berthold Ocker, Pietro Gambardella, and Gilles Gaudin (2014), "Spin-orbit torque magnetization switching of a three-terminal perpendicular magnetic tunnel junction," Applied Physics Letters 104, 042406.

Cubukcu, Murat, Olivier Boulle, Nikolaï Mikuszeit, and Claire Hamelin (2015), "Ultra-fast magnetization reversal of a three-terminal perpendicular magnetic tunnel junction by spin-orbit torque," arXiv preprint arXiv:1509.02375.

Decker, M M, M. S. Wörnle, A. Meisinger, M. Vogel, H. S. Körner, G. Y. Shi, C. Song, M. Kronseder, and C. H. Back (2017), "Time Resolved Measurements of the Switching Trajectory of Pt/Co Elements Induced by Spin-Orbit Torques," Physical Review Letters 118, 257201.

Demasius, Kai-Uwe, Timothy Phung, Weifeng Zhang, Brian P Hughes, See-Hun Yang, Andrew Kellock, Wei Han, Aakash Pushp, and Stuart S P Parkin (2016), "Enhanced spinorbit torques by oxygen incorporation in tungsten films," Nature Communications 7, 10644.

Demidov, V E, H Ulrichs, S V Gurevich, S. O. Demokritov, V S Tiberkevich, a N Slavin, a Zholud, and S Urazhdin (2014a), "Synchronization of spin Hall nano-oscillators to external microwave signals." Nature Communications 5, 3179 .

Demidov, V E, S. Urazhdin, E. R. J. Edwards, and S. O. Demokritov (2011a), "Wide-range control of ferromagnetic resonance by spin Hall effect," Applied Physics Letters 99, 172501.

Demidov, V E, S. Urazhdin, E. R. J. Edwards, M. D. Stiles, R. D. McMichael, and S. O. Demokritov (2011b), "Control of Magnetic Fluctuations by Spin Current," Physical Review Letters 107, 107204.

Demidov, V E, S. Urazhdin, a. B. Rinkevich, G. Reiss, and S. O. Demokritov (2014b), "Spin Hall controlled magnonic microwaveguides," Applied Physics Letters 104, 152402.

Demidov, V E, Sergei Urazhdin, Henning Ulrichs, Vasyl Tiberkevich, Andrei Slavin, Dietmar Baither, Guido Schmitz, and S. O. Demokritov (2012), "Magnetic nanooscillator driven by pure spin current." Nature Materials 11, 1028-31.

Deorani, Praveen, Jaesung Son, Karan Banerjee, Nikesh Koirala, Matthew Brahlek, Seongshik Oh, and Hyunsoo Yang (2014), "Observation of inverse spin Hall effect in bismuth selenide," Physical Review B 90, 094403.

Devolder, T, J. Hayakawa, K. Ito, H. Takahashi, S. Ikeda, P. Crozat, N. Zerounian, Joo Von Kim, C. Chappert, and H. Ohno (2008), "Single-shot time-resolved measure- 
ments of nanosecond-scale spin-transfer induced switching: Stochastic versus deterministic aspects," Physical Review Letters 100 (5), 057206.

Duan, Zheng, Carl T. Boone, Xiao Cheng, Ilya N. Krivorotov, Nathalie Reckers, Sven Stienen, Michael Farle, and Jürgen Lindner (2014a), "Spin-wave modes in permalloy/platinum wires and tuning of the mode damping by spin Hall current," Physical Review B 90, 024427.

Duan, Zheng, Andrew Smith, Liu Yang, Brian Youngblood, Jürgen Lindner, Vladislav E. Demidov, Sergej O. Demokritov, and Ilya N. Krivorotov (2014b), "Nanowire spin torque oscillator driven by spin orbit torques," Nature Communications 5, 5616.

Dyakonov, MI, and V. I. Perel (1971), "Current-induced spin orientation of electrons in semiconductors," Physics Letters 35 (6), 459-460.

Dyrdał, A, and J. Barnas (2015), "Current-induced spin polarization and spin-orbit torque in graphene," Physical Review B 92, 165404.

Dzyaloshinskii, IE (1957), "Thermodynamic theory of weak ferromagnetism in antiferromagnetic substances," Sov. Phys. JETP 5, 1259.

Edelstein, V M (1990), "Spin Polarization of conduction electrons induced by electric current in two-dimensional asymmetric electron systems," Solid State Communications 73 (3), 233-235.

Emori, Satoru, Uwe Bauer, Sung-Min Ahn, Eduardo Martinez, and Geoffrey S D Beach (2013), "Current-driven dynamics of chiral ferromagnetic domain walls." Nature Materials 12, 611-6.

Emori, Satoru, Uwe Bauer, Seonghoon Woo, and Geoffrey S D Beach (2014), "Large voltage-induced modification of spin-orbit torques in Pt/Co/GdOx," Applied Physics Letters 105, 222401.

Emori, Satoru, Tianxiang Nan, Amine M Belkessam, Xinjun Wang, Alexei D. Matyushov, Christopher J Babroski, Yuan Gao, Hwaider Lin, and Nian X Sun (2016), "Interfacial spin-orbit torque without bulk spin-orbit coupling," Physical Review B 93, 180402.

Endo, M, F. Matsukura, and H. Ohno (2010), "Current induced effective magnetic field and magnetization reversal in uniaxial anisotropy (Ga,Mn)As," Applied Physics Letters 97 (22), 222501.

Engel, H-A, Bertrand Halperin, and Emmanuel Rashba (2005), "Theory of Spin Hall Conductivity in n-Doped GaAs," Physical Review Letters 95, 166605.

Fan, Xin, Halise Celik, Jun Wu, Chaoying Ni, Kyung-Jin Lee, Virginia O Lorenz, and John Q Xiao (2014a), "Quantifying interface and bulk contributions to spin-orbit torque in magnetic bilayers." Nature Communications 5, 3042.

Fan, Xin, Jun Wu, Yunpeng Chen, Matthew J. Jerry, Huaiwu Zhang, and John Q. Xiao (2013), "Observation of the nonlocal spin-orbital effective field," Nature Communications 4, 1799 .

Fan, Yabin, Xufeng Kou, Pramey Upadhyaya, Qiming Shao, Lei Pan, Murong Lang, Xiaoyu Che, Jianshi Tang, Mohammad Montazeri, Koichi Murata, Li-Te Chang, Mustafa Akyol, Guoqiang Yu, Tianxiao Nie, Kin L. Wong, Jun Liu, Yong Wang, Yaroslav Tserkovnyak, and Kang L. Wang (2016), "Electric-field control of spin-orbit torque in a magnetically doped topological insulator," Nature Nanotechnology 11 (4), 352.

Fan, Yabin, Pramey Upadhyaya, Xufeng Kou, Murong Lang, So Takei, Zhenxing Wang, Jianshi Tang, Liang He, Li-
Te Chang, Mohammad Montazeri, Guoqiang Yu, Wanjun Jiang, Tianxiao Nie, Robert N Schwartz, Yaroslav Tserkovnyak, and Kang L Wang (2014b), "Magnetization switching through giant spin-orbit torque in a magnetically doped topological insulator heterostructure." Nature Materials 13, 699-704.

Fan, Yabin, and Kang L. Wang (2016), "Spintronics Based on Topological Insulators," Spin 06 (02), 1640001.

Fang, D, H. Kurebayashi, J. Wunderlich, K Výborný, Liviu P Zârbo, R. P. Campion, A Casiraghi, B. L. Gallagher, T Jungwirth, and A. J. Ferguson (2011), "Spin-orbitdriven ferromagnetic resonance." Nature Nanotechnology $6(7), 413-7$.

Fert, A (1990), "Magnetic and Transport Properties of Metallic Multilayers," Mater. Sci. Forum 59-60, 439.

Fert, Albert, Vincent Cros, and Joao Sampaio (2013), "Skyrmions on the track," Nature Nanotechnology 8, 152156.

Fert, Albert, Nicolas Reyren, and Vincent Cros (2017), "Magnetic skyrmions : advances in physics and potential applications," Nature Reviews Materials 2, 17031.

Finocchio, G, M. Carpentieri, E. Martinez, and B. Azzerboni (2013), "Switching of a single ferromagnetic layer driven by spin Hall effect," Applied Physics Letters 102 (21), 212410.

Fischer, Mark H, Abolhassan Vaezi, Aurelien Manchon, and Eun-ah Kim (2016), "Spin-torque generation in topological insulator based heterostructures," Physical Review B 93, 125303.

Forster, RH, G.B. Johnston, and D.A. Wheeler (1968), "Studies on the heusler alloysIII. The antiferro-magnetic phase in the $\mathrm{Cu}-\mathrm{Mn}-\mathrm{Sb}$ system," Journal of Physics and Chemistry of Solids 29 (5), 855-861.

Franken, J H, M Herps, H J M Swagten, and B Koopmans (2014), "Tunable chiral spin texture in magnetic domainwalls." Scientific reports 4, 5248 .

Freimuth, Frank, Stefan Blügel, and Yuriy Mokrousov (2010), "Anisotropic Spin Hall Effect from First Principles," Physical Review Letters 105 (24), 246602.

Freimuth, Frank, Stefan Blügel, and Yuriy Mokrousov (2014a), "Berry phase theory of Dzyaloshinskii-Moriya interaction and spin-orbit torques." Journal of Physics: Condensed Matter 26, 104202.

Freimuth, Frank, Stefan Blügel, and Yuriy Mokrousov (2014b), "Spin-orbit torques in $\mathrm{Co} / \mathrm{Pt}(111)$ and Mn/W(001) magnetic bilayers from first principles," Physical Review B 90, 174423.

Freimuth, Frank, Stefan Blügel, and Yuriy Mokrousov (2015), "Direct and inverse spin-orbit torques," Physical Review B 92, 064415.

Freimuth, Frank, Stefan Blügel, and Yuriy Mokrousov (2017), "Spin-orbit torques and tunable DzyaloshinskiiMoriya interaction in $\mathrm{Co} / \mathrm{Cu} / \mathrm{Co}$ trilayers," arXiv preprint arXiv:1711.06102 .

Fuchs, K (1938), "The conductivity of thin metallic films according to the electron theory of metals," Mathematical Proceedings of the Cambridge Philosophical Society 34 (1), 100-108.

Fujimoto, Junji, and Hiroshi Kohno (2014), "Transport properties of Dirac ferromagnet," Physical Review B 90, 214418.

Fukami, S, T Anekawa, C Zhang, and H Ohno (2016a), "A spin-orbit torque switching scheme with collinear magnetic easy axis and current configuration." Nature Nanotechnology $11,621$. 
Fukami, Shunsuke, and Hideo Ohno (2017), "Magnetization switching schemes for nanoscale three-terminal spintronics devices," Japanese Journal of Applied Physics 56 (8), 0802A1.

Fukami, Shunsuke, Chaoliang Zhang, Samik DuttaGupta, and Hideo Ohno (2016b), "Magnetization switching by spin-orbit torque in an antiferromagnet/ferromagnet bilayer system," Nature Materials 15, 535.

Gambardella, Pietro, and Ioan Mihai Miron (2011), "Current-induced spin-orbit torques." Philosophical transactions. Series A, Mathematical, physical, and engineering sciences 369 (1948), 3175-97.

Ganichev, S, E. Ivchenko, S. Danilov, J. Eroms, W. Wegscheider, D. Weiss, and W. Prettl (2001), "Conversion of Spin into Directed Electric Current in Quantum Wells," Physical Review Letters 86, 4358.

Ganichev, S D, V. Bel'kov, L. Golub, E. Ivchenko, Petra Schneider, S. Giglberger, J. Eroms, J. De Boeck, G. Borghs, W. Wegscheider, D. Weiss, and W. Prettl (2004a), "Experimental Separation of Rashba and Dresselhaus Spin Splittings in Semiconductor Quantum Wells," Physical Review Letters 92, 256601.

Ganichev, S D, S. N. Danilov, Petra Schneider, V. V. Bel'kov, L. E. Golub, W. Wegscheider, D. Weiss, and W. Prettl (2004b), "Can an electric current orient spins in quantum wells?" arXiv preprint arXiv:cond-mat/0403641 .

Ganichev, S D, E. L. Ivchenko, V. V. Bel'kov, S. A. Tarasenko, M. Sollinger, D. Weiss, W. Wegscheider, and W. Prettl (2002), "Spin-galvanic effect," Nature 417, 153-156.

Garate, Ion, and M. Franz (2010), "Inverse Spin-Galvanic Effect in the Interface between a Topological Insulator and a Ferromagnet," Physical Review Letters 104, 146802.

Garate, Ion, and A. H. MacDonald (2009), "Influence of a transport current on magnetic anisotropy in gyrotropic ferromagnets," Physical Review B 80, 134403.

Garello, Kevin, Can Onur Avci, Ioan Mihai Miron, Manuel Baumgartner, Abhijit Ghosh, S. Auffret, Olivier Boulle, Gilles Gaudin, and Pietro Gambardella (2014), "Ultrafast magnetization switching by spin-orbit torques," Applied Physics Letters 105, 212402.

Garello, Kevin, Abhijit Ghosh, Can Onur Avci, and Pietro Gambardella (2017), "Unpublished," Unpublished.

Garello, Kevin, Ioan Mihai Miron, Can Onur Avci, Frank Freimuth, Yuriy Mokrousov, Stefan Blügel, S. Auffret, Olivier Boulle, Gilles Gaudin, and Pietro Gambardella (2013), "Symmetry and magnitude of spin-orbit torques in ferromagnetic heterostructures." Nature Nanotechnology 8, 587-93.

Géranton, Guillaume, Frank Freimuth, Stefan Blügel, and Yuriy Mokrousov (2015), "Spin-orbit torques in L10$\mathrm{FePt} / \mathrm{Pt}$ thin films driven by electrical and thermal currents," Physical Review B 91, 014417.

Géranton, Guillaume, Bernd Zimmermann, Nguyen H. Long, Phivos Mavropoulos, Stefan Blügel, Frank Freimuth, and Yuriy Mokrousov (2016), "Spin-orbit torques and spin accumulation in $\mathrm{FePt} / \mathrm{Pt}$ and $\mathrm{Co} / \mathrm{Cu}$ thin films from first principles: The role of impurities," Physical Review B 93, 224420.

Ghosh, Abhijit, Kevin Garello, Can Onur Avci, Mihai Gabureac, and Pietro Gambardella (2017), "Interface-Enhanced Spin-Orbit Torques and Current-Induced Magnetization Switching of Pd/Co/AlOx Layers," Physical Review Applied 7, 014004.
Ghosh, S, and A. Manchon (2017a), "Spin-orbit torque in two-dimensional antiferromagnetic topological insulators," Physical Review B 95, 035422.

Ghosh, Sumit, and Aurelien Manchon (2017b), "Spin-orbit torque in 3D topological insulator-ferromagnet heterostructure: crossover between bulk and surface transport," arXiv preprint arXiv:1711.11016v1 .

Gomonay, E V, and Vadim Loktev (2014), "Spintronics of antiferromagnetic systems (Review Article)," Low Temperature Physics 40, 17-35.

Gomonay, Helen V, Roman V. Kunitsyn, and Vadim M. Loktev (2012), "Symmetry and the macroscopic dynamics of antiferromagnetic materials in the presence of spinpolarized current," Physical Review B 85, 134446.

Gomonay, Helen V, and Vadim M. Loktev (2010), "Spin transfer and current-induced switching in antiferromagnets," Physical Review B 81, 144427.

Gomonay, Olena, T. Jungwirth, and J. Sinova (2016), "High Antiferromagnetic Domain Wall Velocity Induced by Néel Spin-Orbit Torques," Physical Review Letters 117, 017202.

Gorchon, J, S. Bustingorry, J. Ferré, V. Jeudy, a. B. Kolton, and T. Giamarchi (2014), "Pinning-Dependent Field-Driven Domain Wall Dynamics and Thermal Scaling in an Ultrathin $\mathrm{Pt} / \mathrm{Co} / \mathrm{Pt}$ Magnetic Film," Physical Review Letters 113, 027205.

Gould, C, C. Rüster, T. Jungwirth, E. Girgis, G. Schott, R. Giraud, K. Brunner, G. Schmidt, and L. Molenkamp (2004), "Tunneling Anisotropic Magnetoresistance: A Spin-Valve-Like Tunnel Magnetoresistance Using a Single Magnetic Layer," Physical Review Letters 93, 117203.

Grimmer, H (1993), "General relations for transport properties in magnetically ordered crystals," Acta Crystallographica Section A: Foundations of Crystallography 49 (5), 763 771.

Grytsyuk, Sergiy, Abderrezak Belabbes, Paul M. Haney, Hyun Woo Lee, Kyung Jin Lee, M. D. Stiles, Udo Schwingenschlögl, and Aurelien Manchon (2016), "k-asymmetric spin splitting at the interface between transition metal ferromagnets and heavy metals," Physical Review B 93, 174421.

Grzybowski, M J, P. Wadley, K. W. Edmonds, R. Beardsley, V. Hills, R. P. Campion, B. L. Gallagher, J. S. Chauhan, V. Novak, T. Jungwirth, F. Maccherozzi, and S. S. Dhesi (2017), "Imaging current-induced switching of antiferromagnetic domains in CuMnAs," Physical Review Letters 118, 057701.

Guo, Wei, Guillaume Prenat, and Bernard Dieny (2014), "A novel architecture of non-volatile magnetic arithmetic logic unit using magnetic tunnel junctions," Journal of Physics D: Applied Physics 47 (16), 165001.

Haazen, P P J, E Murè, J H Franken, R Lavrijsen, H J M Swagten, and B Koopmans (2013), "Domain wall depinning governed by the spin Hall effect." Nature Materials 12, 299-303.

Hahn, Christian, Georg Wolf, Bartek Kardasz, Steve Watts, Mustafa Pinarbasi, and Andrew D. Kent (2016), "TimeResolved Studies of the Spin-Transfer Reversal Mechanism in Perpendicularly Magnetized Magnetic Tunnel Junctions," Physical Review B 94, 214432.

Hals, Kjetil, and Arne Brataas (2013), "Phenomenology of current-induced spin-orbit torques," Physical Review B 88, 085423.

Hals, Kjetil, Arne Brataas, and Yaroslav Tserkovnyak (2010), "Scattering theory of charge-currentinduced magnetization 
dynamics," Europhysics Letters 90, 47002.

Hamadeh, A, O Allivy Kelly, C. Hahn, H Meley, R Bernard, A H Molpeceres, V V Naletov, A. Anane, V Cros, S O Demokritov, J L Prieto, M Mu, G De Loubens, and O Klein (2014), "Electronic control of the spin-wave damping in a magnetic insulator," Physical Review Letters 113, 197203.

Han, Jiahao, A Richardella, Saima A Siddiqui, Joseph Finley, N Samarth, and Luqiao Liu (2017), "Room-Temperature Spin-Orbit Torque Switching Induced by a Topological Insulator," Physical Review Letters 119, 077702.

Haney, Paul M, R A Duine, A.S. Núñez, and A. H. MacDonald (2008), "Current-induced torques in magnetic metals: Beyond spin-transfer," Journal of Magnetism and Magnetic Materials 320, 1300-1311.

Haney, Paul M, Hyun Woo Lee, Kyung Jin Lee, Aurélien Manchon, and M. D. Stiles (2013a), "Current-induced torques and interfacial spin-orbit coupling," Physical Review B 88, 214417.

Haney, Paul M, and M. D. Stiles (2010), "Current-Induced Torques in the Presence of Spin-Orbit Coupling," Physical Review Letters 105, 126602.

Haney, PM, H.-W. Lee, K.-J. Lee, A. Manchon, and M.D. Stiles (2013b), "Current induced torques and interfacial spin-orbit coupling: Semiclassical modeling," Physical Review B 87, 174411.

Hao, Qiang, and Gang Xiao (2015), "Giant Spin Hall Effect and Switching Induced by Spin-Transfer Torque in a W/Co40Fe40B20/MgO Structure with Perpendicular Magnetic Anisotropy," Physical Review Applied 3, 034009.

Harder, M, Z. X. Cao, Y. S. Gui, X. L. Fan, and C.-M. Hu (2011), "Analysis of the line shape of electrically detected ferromagnetic resonance," Physical Review B 84, 054423.

Harder, Michael, Yongsheng Gui, and Can-ming Hu (2016), "Electrical detection of magnetization dynamics via spin rectification effects," Physics Reports 661, 1-59.

Hasan, M Z, and C Kane (2010), "Colloquium: Topological insulators," Reviews of Modern Physics 82, 3045-3067.

Hasan, M Zahid, and Joel E. Moore (2011), "ThreeDimensional Topological Insulators," Annual Review of Condensed Matter Physics 2, 55-78.

Hayashi, Masamitsu, Junyeon Kim, Michihiko Yamanouchi, and Hideo Ohno (2014), "Quantitative characterization of the spin-orbit torque using harmonic Hall voltage measurements," Physical Review B 89, 144425.

Heide, M, G. Bihlmayer, and Stefan Blügel (2008), "Dzyaloshinskii-Moriya interaction accounting for the orientation of magnetic domains in ultrathin films: Fe/W(110)," Physical Review B 78, 140403.

Hellman, Frances, Axel Hoffmann, Yaroslav Tserkovnyak, Geoffrey S. D. Beach, Eric E. Fullerton, Chris Leighton, Allan H. MacDonald, Daniel C. Ralph, Dario A. Arena, Hermann A. Dürr, Peter Fischer, Julie Grollier, Joseph P. Heremans, Tomas Jungwirth, Alexey V. Kimel, Bert Koopmans, Ilya N. Krivorotov, Steven J. May, Amanda K. Petford-Long, James M. Rondinelli, Nitin Samarth, Ivan K. Schuller, Andrei N. Slavin, Mark D. Stiles, Oleg Tchernyshyov, André Thiaville, and Barry L. Zink (2017), "Interface-induced phenomena in magnetism," Reviews of Modern Physics 89 (2), 025006.

Herring, C (1966), "Magnetism: Exchange interactions among itinerant electrons," in Magnetism, edited by George Tibor Rado and Harry Suhl (Academic Press) p. 407.
Hibino, Y, T. Hirai, K. Hasegawa, T. Koyama, and D. Chiba (2017), "Enhancement of the spin-orbit torque in a Pt/Co system with a naturally oxidized Co layer," Applied Physics Letters 111 (13), 132404.

Honolka, J, A. A. Khajetoorians, V. Sessi, T. O. Wehling, S. Stepanow, J. L. Mi, B. B. Iversen, T. Schlenk, J. Wiebe, N. B. Brookes, A. I. Lichtenstein, Ph Hofmann, K. Kern, and R. Wiesendanger (2012), "In-plane magnetic anisotropy of Fe atoms on Bi2Se3(111)," Physical Review Letters 108, 256811.

Hou, Dazhi, Z. Qiu, K. Harii, Y. Kajiwara, K. Uchida, Y. Fujikawa, H. Nakayama, T. Yoshino, T. An, K. Ando, Xiaofeng Jin, and E. Saitoh (2012), "Interface induced inverse spin Hall effect in bismuth/permalloy bilayer," Applied Physics Letters 101 (4), 042403.

Hrabec, Ales, Joao Sampaio, Mohamed Belmeguenai, Isabell Gross, Raphael Weil, Salim Mourad Chérif, Andrei Stachkevitch, Vincent Jacques, Andre Thiaville, and Stanislas Rohart (2017), "Current-induced skyrmion generation and dynamics in symmetric bilayers," Nature Communications 8, 15765 .

Hubert, A, and R. Schäfer (1998), Magnetic Domains (Springer Verlag, Berlin).

Hupfauer, T, A Matos-Abiague, M Gmitra, F Schiller, J Loher, D Bougeard, C H Back, J Fabian, and D Weiss (2015), "Emergence of spinorbit fields in magnetotransport of quasi-two-dimensional iron on gallium arsenide," Nature Communications 6, 7374.

Inoue, Jun-ichiro, G. E. W. Bauer, and Laurens Molenkamp (2004), "Suppression of the persistent spin Hall current by defect scattering," Physical Review B 70, 041303.

Inoue, Jun-ichiro, Gerrit E. W. Bauer, and Laurens W. Molenkamp (2003), "Diffuse transport and spin accumulation in a Rashba two-dimensional electron gas," Physical Review B 67, 033104.

Isasa, Miren, M. Carmen Martínez-Velarte, Estitxu Villamor, César Magén, Luis Morellón, José M. De Teresa, M. Ricardo Ibarra, Giovanni Vignale, Evgueni V. Chulkov, Eugene E. Krasovskii, Luis E. Hueso, and Fèlix Casanova (2016), "Origin of inverse Rashba-Edelstein effect detected at the $\mathrm{Cu} / \mathrm{Bi}$ interface using lateral spin valves," Physical Review B 93, 014420.

Ivchenko, E L, and S. D. Ganichev (2008), "Spinphotogalvanic," in Spin Physics in Semiconductors, edited by M.I. Dyakonov (Springer, New York) p. 245.

Ivchenko, E L, Y. B. Lyanda-Geller, and G. E. Pikus (1989), "Photocurrent in structures with quantum wells with an optical orientation of free carriers," JETP Lett. 50 (3), 175-177.

Ivchenko, E L, and G. E. Pikus (1978), "New photogalvanic effect in gyrotropic crystals," Pis'ma Zh. Eksp. Teor. Fiz 27, 604 .

Jabeur, Kotb, G Di Pendina, F Bernard-Granger, and G Prenat (2014), "Spin Orbit Torque Non-Volatile Flip-Flop for High Speed and Low Energy Applications," Electron Device Letters, IEEE 35 (3), 408-410.

Jamali, Mahdi, Joon Sue Lee, Jong Seok Jeong, Farzad Mahfouzi, Yang Lv, Zhengyang Zhao, Branislav Nikolic, K. Andre Mkhoyan, Nitin Samarth, and Jian-Ping Wang (2015), "Giant Spin Pumping and Inverse Spin Hall Effect in the Presence of Surface Spin-Orbit Coupling of Topological Insulator Bi2Se3," Nano Letters 15, 7126.

Jamali, Mahdi, Kulothungasagaran Narayanapillai, Xuepeng Qiu, Li Ming Loong, Aurelien Manchon, and Hyun- 
soo Yang (2013), "Spin-orbit torques in Co/Pd multilayer nanowires," Physical Review Letters 111, 246602.

Jiang, Wanjun, Gong Chen, Kai Liu, Jiadong Zang, Suzanne G.E. te Velthuis, and Axel Hoffmann (2017a), "Skyrmions in magnetic multilayers," Physics Reports 704, $1-49$.

Jiang, Wanjun, Pramey Upadhyaya, Wei Zhang, Guoqiang Yu, M Benjamin Jungfleisch, Frank Y Fradin, John E Pearson, Yaroslav Tserkovnyak, Kang L Wang, Olle Heinonen, Suzanne G E Velthuis, and Axel Hoffmann (2015), "Blowing magnetic skyrmion bubbles," Science 349, 283.

Jiang, Wanjun, Xichao Zhang, Guoqiang Yu, Wei Zhang, M. Benjamin Jungfleisch, John E. Pearson, Olle Heinonen, Kang L. Wang, Yan Zhou, Axel Hoffmann, and Suzanne G. E. te Velthuis (2017b), "Direct Observation of the Skyrmion Hall Effect," Nature Physics 13, 162.

Jonietz, F, S Mühlbauer, C Pfleiderer, A Neubauer, W Münzer, A Bauer, T. Adams, R Georgii, P Böni, R a Duine, Karin Everschor, M Garst, and A Rosch (2010), "Spin transfer torques in MnSi at ultralow current densities." Science (New York, N.Y.) 330, 1648.

Jué, Emilie, C K Safeer, Marc Drouard, Alexandre Lopez, and Paul Balint (2016), "Chiral damping of magnetic domain walls," Nature Materials 15, 272.

Jungfleisch, M B, W. Zhang, J. Sklenar, J. Ding, W. Jiang, H. Chang, F. Y. Fradin, J. E. Pearson, J. B. Ketterson, V. Novosad, M. Wu, and A. Hoffmann (2016), "Large SpinWave Bullet in a Ferrimagnetic Insulator Driven by the Spin Hall Effect," Physical Review Letters 116, 057601.

Jungwirth, T, X Marti, P Wadley, and J Wunderlich (2016), "Antiferromagnetic spintronics," Nature Nanotechnology 11, 231.

Kajiwara, Y, K. Harii, S. Takahashi, Jun-ichiro Ohe, K. Uchida, M Mizuguchi, H Umezawa, H Kawai, K. Ando, K Takanashi, S. Maekawa, and E. Saitoh (2010), "Transmission of electrical signals by spin-wave interconversion in a magnetic insulator." Nature 464 (7286), 262-6.

Kane, C L, and E. J. Mele (2005), "Quantum Spin Hall Effect in Graphene," Physical Review Letters 95, 226801.

Kang, Wang, Zhaohao Wang, Youguang Zhang, JacquesOlivier Klein, Weifeng Lv, and Weisheng Zhao (2016), "Spintronic logic design methodology based on spin Hall effectdriven magnetic tunnel junctions," Journal of Physics D: Applied Physics 49, 065008.

Karube, Shutaro, Kouta Kondou, and Yoshi Chika Otani (2016), "Experimental observation of spin-to-charge current conversion at non-magnetic metal/Bi2O3 interfaces," Applied Physics Express 9 (3), 033001.

Kato, Y K, R. Myers, A. Gossard, and D. D. Awschalom (2004a), "Current-Induced Spin Polarization in Strained Semiconductors," Physical Review Letters 93, 176601.

Kato, Y K, R. C. Myers, A. C. Gossard, and D. D. Awschalom (2004b), "Observation of the spin Hall effect in semiconductors." Science (New York, N.Y.) 306, 19101913.

Kent, Andrew D, and Daniel C Worledge (2015), "A new spin on magnetic memories," Nature Nanotechnology 10 (3), 187-191.

Khvalkovskiy, A V, V. Cros, D. Apalkov, V. Nikitin, M. Krounbi, K. a. Zvezdin, A. Anane, J. Grollier, and A. Fert (2013), "Matching domain-wall configuration and spin-orbit torques for efficient domain-wall motion," Physical Review B 87, 020402.
Khvalkovskiy, A V, K A Zvezdin, Ya V Gorbunov, V Cros, J Grollier, A Fert, and A K Zvezdin (2009), "High Domain Wall Velocities due to Spin Currents Perpendicular to the Plane," Physical Review Letters 102, 067206.

Kim, Joo Von, and Myoung Woo Yoo (2017), "Current-driven skyrmion dynamics in disordered films," Applied Physics Letters 110, 132404.

Kim, Junyeon, Peng Sheng, Saburo Takahashi, Seiji Mitani, and Masamitsu Hayashi (2016), "Spin Hall Magnetoresistance in Metallic Bilayers," Physical Review Letters 116, 097201.

Kim, Junyeon, Jaivardhan Sinha, Masamitsu Hayashi, Michihiko Yamanouchi, Shunsuke Fukami, Tetsuhiro Suzuki, Seiji Mitani, and Hideo Ohno (2013a), "Layer thickness dependence of the current-induced effective field vector in Ta/CoFeB/MgO." Nature Materials 12, 240-5.

Kim, Junyeon, Jaivardhan Sinha, Seiji Mitani, Masamitsu Hayashi, Saburo Takahashi, S. Maekawa, Michihiko Yamanouchi, and Hideo Ohno (2014), "Anomalous temperature dependence of current-induced torques in $\mathrm{CoFeB} / \mathrm{MgO}$ heterostructures with Ta-based underlayers," Physical Review B 89, 174424.

Kim, Kab-jin, Se Kwon Kim, Yuushou Hirata, Se-hyeok Oh, Takayuki Tono, Duck-ho Kim, Takaya Okuno, Woo Seung Ham, Sanghoon Kim, Gyoungchoon Go, Yaroslav Tserkovnyak, Arata Tsukamoto, Takahiro Moriyama, Kyung-jin Lee, and Teruo Ono (2017a), "Fast domain wall motion in the vicinity of the angular momentum compensation temperature of ferrimagnets," Nature Materials 16, 1187.

Kim, Kyoung-Whan, Hyun-Woo Lee, Kyung-Jin Lee, and M. D. Stiles (2013b), "Chirality from Interfacial Spin-Orbit Coupling Effects in Magnetic Bilayers," Physical Review Letters 111, 216601.

Kim, Kyoung Whan, Kyung Jin Lee, Jairo Sinova, Hyun Woo Lee, and M. D. Stiles (2017b), "Spin-orbit torques from interfacial spin-orbit coupling for various interfaces," Physical Review B 96, 104438.

Kim, Kyoung-Whan, Jung-Hwan Moon, Kyung-Jin Lee, and Hyun-Woo Lee (2012a), "Prediction of Giant Spin Motive Force due to Rashba Spin-Orbit Coupling," Physical Review Letters 108, 217202.

Kim, Kyoung-Whan, Soo-Man Seo, Jisu Ryu, Kyung-Jin Lee, and Hyun-Woo Lee (2012b), "Magnetization dynamics induced by in-plane currents in ultrathin magnetic nanostructures with Rashba spin-orbit coupling," Physical Review B 85, 180404.

Kobs, a, S. Heße, W. Kreuzpaintner, G. Winkler, D. Lott, P. Weinberger, A. Schreyer, and H. P. Oepen (2011), "Anisotropic Interface Magnetoresistance in $\mathrm{Pt} / \mathrm{Co} / \mathrm{Pt}$ Sandwiches," Physical Review Letters 106, 217207.

Kondou, K, R. Yoshimi, A. Tsukazaki, Y. Fukuma, J. Matsuno, K. S. Takahashi, M. Kawasaki, Y. Tokura, and Y. Otani (2015), "Fermi level dependent charge-to-spin current conversion by Dirac surface state of topological insulators," Nature Physics 12, 1027-1032.

Koyama, T, D Chiba, K Ueda, K Kondou, H Tanigawa, S Fukami, T Suzuki, N Ohshima, N Ishiwata, Y Nakatani, K Kobayashi, and T Ono (2011), "Observation of the intrinsic pinning of a magnetic domain wall in a ferromagnetic nanowire," Nature materials 10 (3), 194-197.

Krupin, O, G. Bihlmayer, K. Starke, S. Gorovikov, J. Prieto, K. Döbrich, Stefan Blügel, and G. Kaindl (2005), "Rashba effect at magnetic metal surfaces," Physical Review B 71, 
201403.

Kubota, Hitoshi, Akio Fukushima, Kay Yakushiji, Taro Nagahama, Shinji Yuasa, Koji Ando, Hiroki Maehara, Yoshinori Nagamine, Koji Tsunekawa, David D. Djayaprawira, Naoki Watanabe, and Yoshishige Suzuki (2007), "Quantitative measurement of voltage dependence of spin-transfer torque in $\mathrm{MgO}$-based magnetic tunnel junctions," Nature Physics 4 (1), 37-41.

Kurebayashi, H, Jairo Sinova, D. Fang, A.C. Irvine, T D Skinner, J. Wunderlich, Vít Novák, R. P. Campion, B. L. Gallagher, E K Vehstedt, L P Zârbo, K Výborný, A. J. Ferguson, and T Jungwirth (2014), "An antidamping spin-orbit torque originating from the Berry curvature." Nature Nanotechnology 9 (3), 211-7.

Kwon, Kon Woo, Sri Harsha Choday, Yusung Kim, Xuanyao Fong, Sang Phill Park, and Kaushik Roy (2014), "SHENVFF: Spin hall effect-based nonvolatile flip-flop for power gating architecture," IEEE Electron Device Letters 35 (4), 488-490.

Lau, Derek, Vignesh Sundar, Jian Gang Zhu, and Vincent Sokalski (2016a), "Energetic molding of chiral magnetic bubbles," Physical Review B 94 (6), 060401(R).

Lau, Yong-Chang, Davide Betto, Karsten Rode, J. M. D. Coey, and Plamen Stamenov (2016b), "Spin-orbit torque switching without an external field using interlayer exchange coupling," Nature Nanotechnology 11, 758.

Lavrijsen, R, P P J Haazen, E Murè, J H Franken, J T Kohlhepp, H J M Swagten, B Koopmans, R Lavrijsen, P P J Haazen, and E Mure (2012), "Asymmetric $\mathrm{Pt} / \mathrm{Co} / \mathrm{Pt}$-stack induced sign-control of current-induced magnetic domain-wall creep," Applied Physics Letters 100, 262408.

Lavrijsen, R, D. M. F. Hartmann, A. van den Brink, Y. Yin, B. Barcones, R. a. Duine, M. a. Verheijen, H. J. M. Swagten, and B. Koopmans (2015), "Asymmetric magnetic bubble expansion under in-plane field in $\mathrm{Pt} / \mathrm{Co} / \mathrm{Pt}$ : Effect of interface engineering," Physical Review B 91, 104414.

Lee, Hwang-Rae, Kyujoon Lee, Jaehun Cho, Young-Ha Choi, Chun-Yeol You, Myung-Hwa Jung, Frédéric Bonell, Yoichi Shiota, Shinji Miwa, and Yoshishige Suzuki (2014a), "Spinorbit torque in a bulk perpendicular magnetic anisotropy $\mathrm{Pd} / \mathrm{FePd} / \mathrm{MgO}$ system," Scientific Reports 4, 6548.

Lee, Jae-Ung, Sungmin Lee, Ji Hoon Ryoo, Soonmin Kang, Tae Yun Kim, Pilkwang Kim, Cheol-Hwan Park, Je-geun Park, and Hyeonsik Cheong (2016), "Ising-Type Magnetic Ordering in Atomically Thin FePS3," Nano Letters 16, 7433.

Lee, Ki-Seung, Dongwook Go, Aurélien Manchon, Paul M Haney, M D Stiles, Hyun-Woo Lee, and Kyung-Jin Lee (2015), "Angular dependence of spin-orbit spin-transfer torques," Physical Review B 91, 144401.

Lee, Ki-Seung, Seo-Won Lee, Byoung-Chul Min, and KyungJin Lee (2013), "Threshold current for switching of a perpendicular magnetic layer induced by spin Hall effect," Applied Physics Letters 102, 112410.

Lee, O J, L. Q. Liu, C. F. Pai, Y. Li, H. W. Tseng, P. G. Gowtham, J. P. Park, D. C. Ralph, and R. a. Buhrman (2014b), "Central role of domain wall depinning for perpendicular magnetization switching driven by spin torque from the spin Hall effect," Physical Review B 89, 024418.

Legrand, William, Davide Maccariello, Nicolas Reyren, Karin Garcia, Christoforos Moutafis, Constance MoreauLuchaire, Sophie Collin, Karim Bouzehouane, Vincent
Cros, and Albert Fert (2017), "Room-Temperature Current-Induced Generation and Motion of sub-100 nm Skyrmions," Nano Letters 17, 2703.

Lepadatu, Serban, Henri Saarikoski, Robert Beacham, Maria Jose Benitez, A Moore, Gavin Burnell, Satoshi Sugimoto, Daniel Yesudas, May C Wheeler, Sarnjeet S Dhesi, Damien Mcgrouther, Stephen Mcvitie, Gen Tatara, and Christopher H Marrows (2017), "Synthetic ferrimagnet nanowires with very low critical current density for coupled domain wall motion," Scientific Reports 7, 1640.

Lesne, E, Yu Fu, S. Oyarzun, J. C. Rojas-Sánchez, D. C. Vaz, H. Naganuma, G. Sicoli, J. P. Attané, M. Jamet, E. Jacquet, J. M. George, A. Barthélémy, H. Jaffrès, A. Fert, M. Bibes, and L. Vila (2016), "Highly efficient and tunable spin-to-charge conversion through Rashba coupling at oxide interfaces," Nature Materials 15 (12), 1261-1266.

Levy, P M (1994), "Giant Magnetoresistance in Magnetic Layered and Granular Materials," Solid State Physics 47, 367.

Li, Hang, H Gao, Liviu P Zârbo, K Výborný, Xuhui Wang, Ion Garate, Fatih Dogan, A Cejchan, Jairo Sinova, T Jungwirth, and Aurélien Manchon (2015), "Intraband and interband spin-orbit torques in noncentrosymmetric ferromagnets," Physical Review B 91, 134402.

Li, Hang, and Aurelien Manchon (2016), "Tunable spincharge conversion through topological phase transitions in zigzag nanoribbons," Physical Review B 93, 235317.

Li, Hang, Xuhui Wang, Fatih Doan, and Aurelien Manchon (2013), "Tailoring spin-orbit torque in diluted magnetic semiconductors," Applied Physics Letters 102, 192411.

Li, Hang, Xuhui Wang, and A. Manchon (2016a), "Valleydependent spin-orbit torques in two-dimensional hexagonal crystals," Physical Review B 93, 035417.

Li, Peng, Tao Liu, Houchen Chang, Alan Kalitsov, Wei Zhang, Gyorgy Csaba, Wei Li, Daniel Richardson, August DeMann, Gaurab Rimal, Himadri Dey, J. S. Jiang, Wolfgang Porod, Stuart B. Field, Jinke Tang, Mario C. Marconi, Axel Hoffmann, Oleg Mryasov, and Mingzhong $\mathrm{Wu}$ (2016b), "Spin-orbit torque-assisted switching in magnetic insulator thin films with perpendicular magnetic anisotropy," Nature Communications 7, 12688.

Li, Xiaopeng, W. Vincent Liu, and Leon Balents (2014), "Spirals and Skyrmions in Two Dimensional Oxide Heterostructures," Physical Review Letters 112, 067202.

Lifshits, Maria, and Michel Dyakonov (2009), "Swapping Spin Currents: Interchanging Spin and Flow Directions," Physical Review Letters 103, 186601.

Linder, Jacob (2014), "Improved domain-wall dynamics and magnonic torques using topological insulators," Physical Review B 90, 041412.

Litzius, Kai, Ivan Lemesh, Benjamin Krüger, Lucas Caretta, Kornel Richter, Felix Büttner, Pedram Bassirian, Johannes Förster, Robert M. Reeve, Markus Weigand, Iuliia Bykova, Hermann Stoll, Gisela Schütz, Geoffrey S. D. Beach, and Mathias Kläui (2017), "Skyrmion Hall Effect Revealed by Direct Time-Resolved X-Ray Microscopy," Nature Physics 13, 170.

Liu, Luqiao, O. Lee, T. J. Gudmundsen, D. C. Ralph, and R. A. Buhrman (2012a), "Current-Induced Switching of Perpendicularly Magnetized Magnetic Layers Using Spin Torque from the Spin Hall Effect," Physical Review Letters 109, 096602.

Liu, Luqiao, Takahiro Moriyama, D. C. Ralph, and R. A. Buhrman (2011), "Spin-Torque Ferromagnetic Resonance 
Induced by the Spin Hall Effect," Physical Review Letters 106, 036601.

Liu, Luqiao, Chi-Feng Pai, Y Li, H W Tseng, D. C. Ralph, and R. A. Buhrman (2012b), "Spin-torque switching with the giant spin Hall effect of tantalum." Science 336, 555558.

Liu, Luqiao, Chi-Feng Pai, D. C. Ralph, and R. a. Buhrman (2012c), "Magnetic Oscillations Driven by the Spin Hall Effect in 3-Terminal Magnetic Tunnel Junction Devices," Physical Review Letters 109, 186602.

Liu, R, W. Lim, and S. Urazhdin (2013), "Spectral Characteristics of the Microwave Emission by the Spin Hall NanoOscillator," Physical Review Letters 110, 147601.

Liu, R H, W. L. Lim, and S. Urazhdin (2014a), "Control of current-induced spin-orbit effects in a ferromagnetic heterostructure by electric field," Physical Review B 89, 220409(R).

Liu, T, Y Zhang, J W Cai, and H Y Pan (2014b), "Thermally robust $\mathrm{Mo} / \mathrm{CoFeB} / \mathrm{MgO}$ trilayers with strong perpendicular magnetic anisotropy." Scientific reports 4, 5895.

MacDonald, A H, and M Tsoi (2011), "Antiferromagnetic metal spintronics." Philosophical transactions. Series A, Mathematical, physical, and engineering sciences 369 (1948), 3098-114.

MacNeill, D, G. M. Stiehl, M. H. D. Guimaraes, R. A. Buhrman, J. Park, and D. C. Ralph (2017), "Control of spin-orbit torques through crystal symmetry in WTe2/ferromagnet bilayers," Nature Physics 13, 300.

Mahan, G D (2000), Many-Particle Physics, 3rd ed. (Plenum Publishers, New York).

Mahendra, DC, Roberto Grassi, Jun-Yang Chen, Mahdi Jamali, D. Reifsnyder Hickey, D. Zhang, Zhengyang Zhao, Hongshi Li, P. Quarterman, Yang Lv, Mo Li, Aurelien Manchon, K. Andre Mkhoyan, Tony Low, and JianPing Wang (2017), "Room temperature giant spin-orbit torque due to quantum confinement in sputtered $\operatorname{BixSe}(1-$ x) films," arXiv:1703.03822.

Mahfouzi, Farzad, Naoto Nagaosa, and Branislav K. Nikolic (2012), "Spin-orbit coupling induced spintransfer torque and current polarization in topologicalinsulator/ferromagnet vertical heterostructures," Physical Review Letters 109, 166602.

Mahfouzi, Farzad, Naoto Nagaosa, and Branislav K. Nikolić (2014), "Spin-to-charge conversion in lateral and vertical topological-insulator/ferromagnet heterostructures with microwave-driven precessing magnetization," Physical Review B 90, 115432.

Mahfouzi, Farzad, Branislav K. Nikolic, Son-hsien Chen, and Ching-ray Chang (2010), "Microwave-driven ferromagnettopological-insulator heterostructures: The prospect for giant spin battery effect and quantized charge pump devices," Physical Review B 82, 195440.

Mahfouzi, Farzad, Branislav K. Nikolić, and Nicholas Kioussis (2016), "Antidamping spin-orbit torque driven by spinflip reflection mechanism on the surface of a topological insulator: A time-dependent nonequilibrium Green function approach," Physical Review B 93, 115419.

Malozemoff, AP, and J.C. Slonczewski (1979), Magnetic Domain Walls in Bubble Materials (Academic, New York).

Mal'shukov, A G, and K. A. Chao (2002), "Optoelectric spin injection in semiconductor heterostructures without a ferromagnet," Physical Review B 65, 241308.

Manchon, A (2011a), "Interfacial spin-orbit splitting and current-driven spin torque in anisotropic tunnel junctions,"
Physical Review B 83, 172403.

Manchon, A (2017), "Spin diffusion and torques in disordered antiferromagnets," Journal of Physics Condensed Matter 29, 104002.

Manchon, A, C. Ducruet, L. Lombard, S. Auffret, B. Rodmacq, B. Dieny, S. Pizzini, J. Vogel, V. Uhlir, M. Hochstrasser, and G. Panaccione (2008), "Analysis of oxygen induced anisotropy crossover in $\mathrm{Pt} / \mathrm{Co} / \mathrm{MOx}$ trilayers," Journal of Applied Physics 104, 043914.

Manchon, A, H C Koo, J Nitta, S M Frolov, and R A Duine (2015), "New perspectives for Rashba spin orbit coupling," Nature Materials 14, 871.

Manchon, A, and S. Zhang (2008), "Theory of nonequilibrium intrinsic spin torque in a single nanomagnet," Physical Review B 78, 212405.

Manchon, A, and S. Zhang (2011), "Spin Torque in Magnetic Systems: Theory," in Handbook of Spin Transport and Magnetism, edited by Evgeny Y Tsymbal and Igor Zutic, Chap. 8 (CRC Press, Boca Raton) pp. 157-178.

Manchon, Aurelien (2011b), "Voltage-driven versus currentdriven spin torque in anisotropic tunneling junctions," IEEE Transactions on Magnetics 47, 2735.

Manchon, Aurelien (2012), "Spin Hall effect versus Rashba torque: a Diffusive Approach," arXiv preprint arXiv:1204.4869v1.

Marmolejo-Tejada, Juan Manuel, Kapildeb Dolui, Predrag Lazić, Po Hao Chang, Søren Smidstrup, Daniele Stradi, Kurt Stokbro, and Branislav K. Nikolić (2017), "Proximity Band Structure and Spin Textures on Both Sides of Topological-Insulator/Ferromagnetic-Metal Interface and Their Charge Transport Probes," Nano Letters 17, 56265633.

Martinez, Eduardo (2012), "Micromagnetic analysis of the Rashba field on current-induced domain wall propagation," Journal of Applied Physics 111, 033901.

Martinez, Eduardo, Satoru Emori, and Geoffrey S. D. Beach (2013), "Current-driven domain wall motion along high perpendicular anisotropy multilayers: The role of the Rashba field, the spin Hall effect, and the DzyaloshinskiiMoriya interaction," Applied Physics Letters 103 (7), 072406.

Martinez, Eduardo, Satoru Emori, Noel Perez, Luis Torres, and Geoffrey S. D. Beach (2014), "Current-driven dynamics of Dzyaloshinskii domain walls in the presence of in-plane fields: Full micromagnetic and one-dimensional analysis," Journal of Applied Physics 115, 213909.

Martinez, Eduardo, Luis Torres, Noel Perez, Maria Auxiliadora Hernandez, Victor Raposo, and Simone Moretti (2015), "Universal chiral-triggered magnetization switching in confined nanodots," Scientific Reports 5, 10156.

Maruyama, T, Y. Shiota, T. Nozaki, K. Ohta, N. Toda, M. A. Mizuguchi, TulapurkarA., T. Shinjo, M. Shiraishi, S. Mizukami, Y. Ando, and Y. Suzuki (2009), "Large voltage-induced magnetic anisotropy change in a few atomic layers of iron," Nature Nanotechnology 4, 158.

Matos-Abiague, A, and R L Rodriguez-Suárez (2009), "Spinorbit coupling mediated spin torque in a single ferromagnetic layer," Physical Review B 80, 94424.

McMichael, RD, and M.J. Donahue (1997), "Head to head domain wall structures in thin magnetic strips," IEEE Transactions on Magnetics 33 (5), 4167-4169.

Meckler, S, N. Mikuszeit, A. Preßler, E. Vedmedenko, O. Pietzsch, and R. Wiesendanger (2009), "Real-Space Observation of a Right-Rotating Inhomogeneous Cycloidal Spin 
Spiral by Spin-Polarized Scanning Tunneling Microscopy in a Triple Axes Vector Magnet," Physical Review Letters 103, 157201.

Meinert, Markus, Dominik Graulich, and Tristan MatallaWagner (2017), "Key role of thermal activation in the electrical switching of antiferromagnetic Mn2Au," arXiv preprint arXiv:1706.06983.

Mellnik, A R, J. S. Lee, A. Richardella, J. L. Grab, P. J. Mintun, M. H. Fischer, A. Vaezi, A. Manchon, E.-a. Kim, N. Samarth, and D. C. Ralph (2014), "Spin-transfer torque generated by a topological insulator," Nature 511 (7510), 449-451.

Metaxas, P J, J P Jamet, A Mougin, M Cormier, J Ferré, V Baltz, B Rodmacq, B Dieny, and R L Stamps (2007), "Creep and Flow Regimes of Magnetic Domain-Wall Motion in Ultrathin $\mathrm{Pt} / \mathrm{Co} / \mathrm{Pt}$ Films with Perpendicular Anisotropy," Physical Review Letters 99, 217208.

Mikuszeit, N, O. Boulle, I. M. Miron, K. Garello, P. Gambardella, G. Gaudin, and L. D. Buda-Prejbeanu (2015), "Spin-orbit torque driven chiral magnetization reversal in ultrathin nanostructures," Physical Review B 92, 144424.

Miron, I, P.-J. Zermatten, G. Gaudin, S. Auffret, B. Rodmacq, and A. Schuhl (2009), "Domain Wall Spin Torquemeter," Physical Review Letters 102, 137202.

Miron, Ioan Mihai, Kevin Garello, Gilles Gaudin, PierreJean Zermatten, Marius V Costache, S. Auffret, Sébastien Bandiera, Bernard Rodmacq, Alain Schuhl, and Pietro Gambardella (2011a), "Perpendicular switching of a single ferromagnetic layer induced by in-plane current injection." Nature 476, 189.

Miron, Ioan Mihai, Gilles Gaudin, S. Auffret, Bernard Rodmacq, Alain Schuhl, Stefania Pizzini, Jan Vogel, and Pietro Gambardella (2010), "Current-driven spin torque induced by the Rashba effect in a ferromagnetic metal layer." Nature Materials 9, 230 .

Miron, Ioan Mihai, Thomas Moore, Helga Szambolics, Liliana Daniela Buda-Prejbeanu, S. Auffret, Bernard Rodmacq, Stefania Pizzini, Jan Vogel, Marlio Bonfim, Alain Schuhl, and Gilles Gaudin (2011b), "Fast current-induced domain-wall motion controlled by the Rashba effect." Nature Materials 10, 419.

Mishra, Rahul, Jiawei Yu, Xuepeng Qiu, M Motapothula, T Venkatesan, and Hyunsoo Yang (2017), "Anomalous Current-Induced Spin Torques in Ferrimagnets near Compensation," Physical Review Letters 118, 167201.

Monso, S, B. Rodmacq, S. Auffret, G. Casali, F. Fettar, B. Gilles, B. Dieny, and P. Boyer (2002), "Crossover from in-plane to perpendicular anisotropy in $\mathrm{Pt} / \mathrm{CoFe} / \mathrm{AlOx}$ sandwiches as a function of $\mathrm{Al}$ oxidation: A very accurate control of the oxidation of tunnel barriers," Applied Physics Letters 80, 4157.

Montazeri, Mohammad, Pramey Upadhyaya, Mehmet C. Onbasli, Guoqiang Yu, Kin L. Wong, Murong Lang, Yabin Fan, Xiang Li, Pedram Khalili Amiri, Robert N. Schwartz, Caroline A. Ross, and Kang L. Wang (2015), "Magnetooptical investigation of spinorbit torques in metallic and insulating magnetic heterostructures," Nature Communications 6, 8958 .

Moore, T A, I M Miron, G Gaudin, G Serret, S. Auffret, B Rodmacq, A Schuhl, S Pizzini, J Vogel, and M Bonfim (2008), "High domain wall velocities induced by current in ultrathin $\mathrm{Pt} / \mathrm{Co} / \mathrm{AlOx}$ wires with perpendicular magnetic anisotropy," Applied Physics Letters 93, 262504.
Moore, T A, I. M. Miron, G. Gaudin, G. Serret, S. Auffret, B. Rodmacq, A. Schuhl, S. Pizzini, J. Vogel, and M. Bonfim (2009), "Erratum: High domain wall velocities induced by current in ultrathin $\mathrm{Pt} / \mathrm{Co} / \mathrm{AlOx}$ wires with perpendicular magnetic anisotropy," Applied Physics Letters 95, 179902.

Moras, P, G. Bihlmayer, P. M. Sheverdyaeva, S. K. Mahatha, M. Papagno, J. Sánchez-Barriga, O. Rader, L. Novinec, S. Gardonio, and C. Carbone (2015), "Magnetizationdependent Rashba splitting of quantum well states at the Co/W interface," Physical Review B 91, 195410.

Moreau-Luchaire, C, C. Moutas, N. Reyren, J. Sampaio, C. A. F. Vaz, N. Van Horne, K. Bouzehouane, K. Garcia, C. Deranlot, P. Warnicke, P. Wohlhüter, J.-M. George, M. Weigand, J. Raabe, V. Cros, and A. Fert (2016), "Additive interfacial chiral interaction in multilayers for stabilization of small individual skyrmions at room temperature," Nature Nanotechnology 11, 444.

Moritz, J, B Rodmacq, S Auffret, and B Dieny (2008), "Extraordinary Hall effect in thin magnetic films and its potential for sensors, memories and magnetic logic applications," Journal of Physics D: Applied Physics 41, 135001.

Moriya, Toru (1960), "Anisotropic superexchange interaction and weak ferromagnetism," Physical Review 120, 91.

Murakami, Shuichi, N. Nagaosa, and Shou-Cheng Zhang (2003), "Dissipationless Quantum Spin Current at Room Temperature," Science (New York, N.Y.) 301, 1348-1351.

Nakatani, Yoshinobu, André Thiaville, and Jacques Miltat (2005), "Head-to-head domain walls in soft nano-strips: A refined phase diagram," Journal of Magnetism and Magnetic Materials 290-291 PA, 750-753.

Nakayama, H, Matthias Althammer, Y.-T. Chen, K. Uchida, Y. Kajiwara, D. Kikuchi, T. Ohtani, Stephan Geprägs, M. Opel, S. Takahashi, R. Gross, G. E. W. Bauer, S. T. B. Goennenwein, and E. Saitoh (2013), "Spin Hall Magnetoresistance Induced by a Nonequilibrium Proximity Effect," Physical Review Letters 110, 206601.

Nakayama, Hiroyasu, Yusuke Kanno, Hongyu An, Takaharu Tashiro, Satoshi Haku, Akiyo Nomura, and Kazuya Ando (2016), "Rashba-Edelstein Magnetoresistance in Metallic Heterostructures," Physical Review Letters 117, 116602.

Nan, Tianxiang, Satoru Emori, Carl T. Boone, Xinjun Wang, Trevor M. Oxholm, John G. Jones, Brandon M. Howe, Gail J. Brown, and Nian X. Sun (2015), "Comparison of spin-orbit torques and spin pumping across $\mathrm{NiFe} / \mathrm{Pt}$ and $\mathrm{NiFe} / \mathrm{Cu} / \mathrm{Pt}$ interfaces," Physical Review B 91, 214416.

Ndiaye, Papa B, C. A. Akosa, M. H. Fischer, A. Vaezi, E. A. Kim, and A. Manchon (2017), "Dirac spin-orbit torques and charge pumping at the surface of topological insulators," Physical Review B 96, 014408.

Nguyen, Anh Kiet, Hans Joakim Skadsem, and Arne Brataas (2007), "Giant current-driven domain wall mobility in (Ga,Mn)As," Physical Review Letters 98, 146602.

Nguyen, Minh Hai, Chi Feng Pai, Kayla X. Nguyen, David A. Muller, D. C. Ralph, and R. A. Buhrman (2015), "Enhancement of the anti-damping spin torque efficacy of platinum by interface modification," Applied Physics Letters 106, 222402.

Nguyen, Minh Hai, D. C. Ralph, and R. A. Buhrman (2016), "Spin Torque Study of the Spin Hall Conductivity and Spin Diffusion Length in Platinum Thin Films with Varying Resistivity," Physical Review Letters 116, 126601.

Nistor, C, T. Balashov, J. J. Kavich, A. Lodi Rizzini, B. Ballesteros, G. Gaudin, S. Auffret, B. Rodmacq, S. S. 
Dhesi, and P. Gambardella (2011), "Orbital moment anisotropy of $\mathrm{Pt} / \mathrm{Co} / \mathrm{AlOx}$ heterostructures with strong Rashba interaction," Physical Review B 84, 054464.

Nomura, Kentaro, and Naoto Nagaosa (2011), "SurfaceQuantized Anomalous Hall Current and the Magnetoelectric Effect in Magnetically Disordered Topological Insulators," Physical Review Letters 106, 166802.

Nozaki, T, Y Jiang, Y Kaneko, A Hirohata, N Tezuka, S Sugimoto, and K Inomata (2004), "Spin-dependent quantum oscillations in magnetic tunnel junctions with Ru quantum wells," Physical Review B 70, 172401.

Núñez, AS, RA Duine, Paul Haney, and AH MacDonald (2006), "Theory of spin torques and giant magnetoresistance in antiferromagnetic metals," Physical Review B 73, 214426.

Obata, Katsunori, and G. Tatara (2008), "Current-induced domain wall motion in Rashba spin-orbit system," Physical Review B 77, 214429.

Oboril, Fabian, Rajendra Bishnoi, Mojtaba Ebrahimi, and Mehdi B Tahoori (2015), "Evaluation of Hybrid Memory Technologies Using SOT-MRAM for On-Chip Cache Hierarchy," IEEE Transactions on Magnetics 34 (3), 367-380.

Oh, Se-Chung, Seung-Young Park, Aurélien Manchon, Mairbek Chshiev, Jae-Ho Han, Hyun-Woo Lee, Jang-Eun Lee, Kyung-Tae Nam, Younghun Jo, Yo-Chan Kong, Bernard Dieny, and Kyung-Jin Lee (2009), "Bias-voltage dependence of perpendicular spin-transfer torque in asymmetric MgO-based magnetic tunnel junctions," Nature Physics 5, 898.

Oh, Young-Wan, Seung-Heon Chris Baek, Y M Kim, Hae Yeon Lee, Kyeong-Dong Lee, Chang-Geun Yang, EunSang Park, Ki-Seung Lee, Kyoung-Whan Kim, Gyungchoon Go, Jong-Ryul Jeong, Byoung-Chul Min, Hyun-Woo Lee, Kyung-Jin Lee, and Byong-Guk Park (2016), "Fieldfree switching of perpendicular magnetization through spin-orbit torque in antiferromagnet/ferromagnet/oxide structures." Nature Nanotechnology 11, 878.

Olejník, K, V. Novák, J. Wunderlich, and T. Jungwirth (2015), "Electrical detection of magnetization reversal without auxiliary magnets," Physical Review B 91, 180402(R).

Olejnik, K, V Schuler, X Marti, and V Nova (2017a), "Antiferromagnetic CuMnAs multi-level memory cell with microelectronic compatibility," Nature Communications 8, 15434.

Olejnik, K, T. Seifert, Z. Kaspar, V. Novak, P. Wadley, R. P. Campion, M. Baumgartner, P. Gambardella, P. Nemec, J. Wunderlich, J. Sinova, M. Muller, T. Kampfrath, and T. Jungwirth (2017b), "THz electrical writing speed in an antiferromagnetic memory," arXiv preprint $\operatorname{arXiV:1711.08444~.~}$

Ortiz Pauyac, Christian, Xuhui Wang, Mairbek Chshiev, and Aurelien Manchon (2013), "Angular dependence and symmetry of Rashba spin torque in ferromagnetic heterostructures," Applied Physics Letters 102 (25), 252403.

Ou, Yongxi, Shengjie Shi, D. C. Ralph, and R. A. Buhrman (2016), "Strong spin Hall effect in the antiferromagnet PtMn," Physical Review B 93, 220405(R).

Pai, Chi-Feng, Luqiao Liu, Y. Li, H. W. Tseng, D. C. Ralph, and R. a. Buhrman (2012), "Spin transfer torque devices utilizing the giant spin Hall effect of tungsten," Applied Physics Letters 101, 122404.

Pai, Chi-Feng, Minh-Hai Nguyen, Carina Belvin, Luis Henrique Vilela-Leão, D. C. Ralph, and R. A. Buhrman (2014),
"Enhancement of perpendicular magnetic anisotropy and transmission of spin-Hall-effect-induced spin currents by a Hf spacer layer in W/Hf/CoFeB/MgO layer structures," Applied Physics Letters 104, 082407.

Pai, Chi Feng, Yongxi Ou, Luis Henrique Vilela-Leao, D. C. Ralph, and R. A. Buhrman (2015), "Dependence of the efficiency of spin Hall torque on the transparency of $\mathrm{Pt}$ /ferromagnetic layer interfaces," Physical Review B 92, 064426.

Park, B G, J. Wunderlich, D. a. Williams, S. J. Joo, K. Y. Jung, K. H. Shin, K. Olejník, a. B. Shick, and T. Jungwirth (2008), "Tunneling Anisotropic Magnetoresistance in Multilayer-(Co/Pt)/AlOx/Pt Structures," Physical Review Letters 100, 087204.

Park, Junbo, G. E. Rowlands, O. J. Lee, D. C. Ralph, and R. A. Buhrman (2014), "Macrospin modeling of sub-ns pulse switching of perpendicularly magnetized free layer via spin-orbit torques for cryogenic memory applications," Applied Physics Letters 105, 102404.

Parkin, S S P (1993), "Origin of enhanced magnetoresistance of magnetic multilayers: Spin-dependent scattering from magnetic interface states," Physical Review Letters 71 (10), 1641-1644.

Parkin, SSP (2014), "http://taf.fi/en/millenniumtechnology-prize/winner-2014/,".

Parkin, Stuart, and See-Hun Yang (2015), "Memory on the racetrack," Nature Nanotechnology 10 (3), 195-198.

Parkin, Stuart S P, Masamitsu Hayashi, and Luc Thomas (2008), "Magnetic domain-wall racetrack memory." Science (New York, N.Y.) 320, 190-4.

Pellegren, J P, D. Lau, and V. Sokalski (2017), "Dispersive Stiffness of Dzyaloshinskii Domain Walls," Physical Review Letters 119 (2), 027203.

Perez, N, E. Martinez, L. Torres, S.-H. Woo, S. Emori, and G. S. D. Beach (2014), "Chiral magnetization textures stabilized by the Dzyaloshinskii-Moriya interaction during spin-orbit torque switching," Applied Physics Letters 104 (9), 092403.

Pesin, D A, and A. H. MacDonald (2012a), "Quantum kinetic theory of current-induced torques in Rashba ferromagnets," Physical Review B 86, 014416.

Pesin, D A, and A. H. MacDonald (2012b), "Spintronics and pseudospintronics in graphene and topological insulators." Nature Materials 11, 409.

Pi, Ung Hwan, Kee Won Kim, Ji Young Bae, Sung Chul Lee, Young Jin Cho, Kwang Seok Kim, and Sunae Seo (2010), "Tilting of the spin orientation induced by Rashba effect in ferromagnetic metal layer," Applied Physics Letters 97 (16), 162507.

Pizzini, S, V. Uhlir, J. Vogel, N. Rougemaille, S. Laribi, V. Cros, E. Jimenez, Julio Camarero, Carsten Tieg, Edgar Bonet, Marlio Bonfim, Richard Mattana, C. Deranlot, F. Petroff, C. Ulysse, G. Faini, and A. Fert (2009), "High Domain Wall Velocity at Zero Magnetic Field Induced by Low Current Densities in Spin Valve Nanostripes," Applied Physics Express 2, 023003.

Pizzini, S, J. Vogel, S. Rohart, L. D. Buda-Prejbeanu, E. Jué, O. Boulle, I. M. Miron, C. K. Safeer, S. Auffret, G. Gaudin, and A. Thiaville (2014), "Chirality-Induced Asymmetric Magnetic Nucleation in Pt/Co/AlOx Ultrathin Microstructures," Physical Review Letters 113, 047203.

Pollard, Shawn D, Joseph A Garlow, Jiawei Yu, Zhen Wang, Yimei Zhu, and Hyunsoo Yang (2017), "Observation of stable Néel skyrmions in $\mathrm{Co} / \mathrm{Pd}$ multilayers with Lorentz 
transmission electron microscopy," Nature Communications 8, 14761.

Prenat, Guillaume, Kotb Jabeur, Pierre Vanhauwaert, Gregory Di Pendina, Fabian Oboril, Rajendra Bishnoi, Mojtaba Ebrahimi, Nathalie Lamard, Olivier Boulle, Kevin Garello, Juergen Langer, Berthold Ocker, Marie Claire Cyrille, Pietro Gambardella, Mehdi Tahoori, and Gilles Gaudin (2016), "Ultra-Fast and High-Reliability SOTMRAM: From Cache Replacement to Normally-Off Computing," IEEE Transactions on Multi-Scale Computing Systems 2 (1), 49-60.

Qaiumzadeh, A, R. A. Duine, and M. Titov (2015), "Spinorbit torques in two-dimensional Rashba ferromagnets," Physical Review B 92, 014402.

Qi, Xiao-Liang, Taylor L. Hughes, and Shou-Cheng Zhang (2008), "Topological field theory of time-reversal invariant insulators," Physical Review B 78, 195424.

Qi, Xiao-Liang, and Shou-Cheng Zhang (2011), "Topological insulators and superconductors," Review of Modern Physics 83, 1057-1110.

Qiu, Xuepeng, Praveen Deorani, Kulothungasagaran Narayanapillai, Ki-Seung Lee, Kyung-Jin Lee, Hyun-Woo Lee, and Hyunsoo Yang (2014), "Angular and temperature dependence of current induced spin-orbit effective fields in $\mathrm{Ta} / \mathrm{CoFeB} / \mathrm{MgO}$ nanowires." Scientific reports 4, 4491.

Qiu, Xuepeng, William Legrand, Pan He, Yang Wu, Jiawei Yu, Rajagopalan Ramaswamy, Aurelien Manchon, and Hyunsoo Yang (2016), "Enhanced Spin-Orbit Torque via Modulation of Spin Current Absorption," Physical Review Letters 117, 217206.

Qiu, Xuepeng, Kulothungasagaran Narayanapillai, Yang Wu, Praveen Deorani, Dong-Hyuk Yang, Woo-suk Noh, Jaehoon Park, Kyung-Jin Lee, Hyun-woo Lee, and Hyunsoo Yang (2015), "Spinorbit-torque engineering via oxygen manipulation," Nature Nanotechnology 10, 333-338.

Qiu, Z Q, and S. D. Bader (2000), "Surface magneto-optic Kerr effect," Review of Scientific Instruments 71, 1243.

Ralph, DC, and M.D. Stiles (2008), "Spin transfer torques," Journal of Magnetism and Magnetic Materials 320 (7), $1190-1216$

Ramaswamy, Rajagopalan, Xuepeng Qiu, Tanmay Dutta, Shawn David Pollard, and Hyunsoo Yang (2016), "Hf thickness dependence of spin-orbit torques in $\mathrm{Hf} / \mathrm{CoFeB} / \mathrm{MgO}$ heterostructures," Applied Physics Letters 108 (20), 202406.

Rammer, S, and H. Smith (1986), "Quantum field theoretical methods in transport theory of metals," Reviews of Modern Physics 58, 323.

Ravelosona, D, D. Lacour, J. A. Katine, B. D. Terris, and C. Chappert (2005), "Nanometer scale observation of high efficiency thermally assisted current-driven domain wall depinning," Physical Review Letters 95 (11), 117203.

Reichlova, H, D. Kriegner, V. Holy, K. Olejnik, V. Novak, M. Yamada, K. Miura, S. Ogawa, H. Takahashi, T. Jungwirth, and J. Wunderlich (2015), "Current-induced torques in structures with ultrathin IrMn antiferromagnets," Physical Review B 92, 165424.

Reynolds, Neal, Priyamvada Jadaun, John T Heron, Colin L Jermain, Jonathan Gibbons, Robyn Collette, R A Buhrman, D G Schlom, and D C Ralph (2017), "Spin Hall torques generated by rare-earth thin films," Physical Review B 95, 064412.

Rodmacq, B, A. Manchon, C. Ducruet, S. Auffret, and B. Dieny (2009), "Influence of thermal annealing on the per- pendicular magnetic anisotropy of $\mathrm{Pt} / \mathrm{Co} / \mathrm{AlOx}$ trilayers," Physical Review B 79 (2), 024423.

Rojas-Sánchez, J C, P. Laczkowski, J. Sampaio, S. Collin, K. Bouzehouane, N. Reyren, H. Jaffrès, A. Mougin, and J. M. George (2016), "Perpendicular magnetization reversal in $\mathrm{Pt} /[\mathrm{Co} / \mathrm{Ni}] 3 / \mathrm{Al}$ multilayers via the spin Hall effect of Pt," Applied Physics Letters 108, 082406.

Rojas-Sánchez, J C, L Vila, G Desfonds, S Gambarelli, J.-P. Attane, J M De Teresa, C Magén, and A. Fert (2013), "Spin-to-charge conversion using Rashba coupling at the interface between non-magnetic materials." Nature Communications 4, 2944.

Ryu, KS, L Thomas, S Yang, and Stuart S P Parkin (2012), "Current induced tilting of domain walls in high velocity motion along perpendicularly magnetized micron-sized Co/Ni/Co racetracks," Applied Physics Express 5, 093006.

Ryu, Kwang-Su, Luc Thomas, See-Hun Yang, and Stuart Parkin (2013), "Chiral spin torque at magnetic domain walls." Nature Nanotechnology 8, 527.

Safeer, C K, Emilie Jué, Alexandre Lopez, Liliana BudaPrejbeanu, Stéphane Auffret, Stefania Pizzini, Olivier Boulle, Ioan Mihai Miron, and Gilles Gaudin (2016), "Spin-orbit torque magnetization switching controlled by geometry." Nature Nanotechnology 11, 143.

Saidaoui, Hamed Ben Mohamed, and Aurélien Manchon (2016), "Spin-Swapping Transport and Torques in Ultrathin Magnetic Bilayers," Physical Review Letters 117, 036601.

Saitoh, E, M. Ueda, H. Miyajima, and G. Tatara (2006), "Conversion of spin current into charge current at room temperature: Inverse spin-Hall effect," Applied Physics Letters 88 (18), 182509.

Sakai, Akio, and Hiroshi Kohno (2014), "Spin torques and charge transport on the surface of topological insulator," Physical Review B 89, 165307.

Sambles, J R (1983), "The resistivity of thin metal films some critical remarks," Thin Solid Films 106, 321-331.

Sampaio, J, V Cros, S Rohart, A Thiaville, and A Fert (2013), "Nucleation, stability and current-induced motion of isolated magnetic skyrmions in nanostructures." Nature Nanotechnology 8, 839.

Sankey, J C, P. M. Braganca, a. G F Garcia, I. N. Krivorotov, R. a. Buhrman, and D. C. Ralph (2006), "Spintransfer-driven ferromagnetic resonance of individual nanomagnets," Physical Review Letters 96, 227601.

Sankey, Jack C, Yong-Tao Cui, Jonathan Z. Sun, John C. Slonczewski, R. A. Buhrman, and Daniel C. Ralph (2007), "Measurement of the spin-transfer-torque vector in magnetic tunnel junctions," Nature Physics 4, 67.

Scholz, M R, J. Sánchez-Barriga, D. Marchenko, A. Varykhalov, A. Volykhov, L. V. Yashina, and O. Rader (2012), "Tolerance of topological surface states towards magnetic moments: Fe on Bi2Se3," Physical Review Letters 108, 256810.

Schreier, Michael, Takahiro Chiba, Arthur Niedermayr, Johannes Lotze, Hans Huebl, Stephan Geprägs, Saburo Takahashi, Gerrit E W Bauer, Rudolf Gross, and Sebastian T B Goennenwein (2015), "Current-induced spin torque resonance of a magnetic insulator," Physical Review B 92, 144411.

Schryer, NL, and L.R. Walker (1974), "The motion of 180 domain walls in uniform dc magnetic fields," Journal of Applied Physics 45 (12), 5406-5421. 
Seo, Soo-Man, Kyoung-Whan Kim, Jisu Ryu, Hyun-Woo Lee, and Kyung-Jin Lee (2012), "Current-induced motion of a transverse magnetic domain wall in the presence of spin Hall effect," Applied Physics Letters 101 (2), 022405.

Shao, Qiming, Guoqiang Yu, Yann-wen Lan, Yumeng Shi, Ming-Yang Li, Cheng Zheng, Xiaodan Zhu, Lain-Jong Li, Pedram Khalili Amiri, and Kang L. Wang (2016), "Strong Rashba-Edelstein Effect-Induced Spin-Orbit Torques in Monolayer Transition Metal Dichalcogenide/Ferromagnet Bilayers," Nano Letters 16, 7514.

Shchelushkin, R, and Arne Brataas (2005), "Spin Hall effects in diffusive normal metals," Physical Review B 71, 045123.

Shen, Ka, R Raimondi, and G Vignale (2015), "Spin current swapping in the two dimensional electron gas," arXiv preprint arXiv:1502.01128v1.

Shi, Shengjie, Yongxi Ou, S. V. Aradhya, D. C. Ralph, and R. A. Buhrman (2017), "Fast, low-current spin-orbit torque switching of magnetic tunnel junctions through atomic modifications of the free layer interfaces," arXiv preprint arXiv:1710.06391 .

Shiino, Takayuki, Se-hyeok Oh, Paul M Haney, Seo-won Lee, Gyungchoon Go, Byong-guk Park, and Kyung-jin Lee (2016), "Antiferromagnetic Domain Wall Motion Driven by Spin-Orbit Torques," Physical Review Letters 117, 087203.

Shiomi, Y, K. Nomura, Y. Kajiwara, K. Eto, M. Novak, Kouji Segawa, Yoichi Ando, and E. Saitoh (2014), "SpinElectricity Conversion Induced by Spin Injection into Topological Insulators," Physical Review Letters 113, 196601.

Shiota, Yoichi, Takayuki Nozaki, Frédéric Bonell, Shinichi Murakami, Teruya Shinjo, and Yoshishige Suzuki (2012), "Induction of coherent magnetization switching in a few atomic layers of FeCo using voltage pulses," Nature Materials $\mathbf{1 1}, 39-43$.

Silov, A Yu, P. A. Blajnov, J. H. Wolter, R. Hey, K. H. Ploog, and N. S. Averkiev (2004), "Current-induced spin polarization at a single heterojunction," Applied Physics Letters 85 (24), 5929.

Sinova, Jairo, Dimitrie Culcer, Qian Niu, N. A. Sinitsyn, T. Jungwirth, and A. H. MacDonald (2004), "Universal intrinsic spin Hall effect," Physical Review Letters 92, 126603.

Sinova, Jairo, and Tomas Jungwirth (2017), "Surprises from the spin Hall effect," Physics Today 70, 38.

Sinova, Jairo, Sergio O. Valenzuela, J. Wunderlich, C. H. Back, and T. Jungwirth (2015), "Spin Hall effect," Review of Modern Physics. 87, 1213.

Skinner, T D, K. Olejnik, L. K. Cunningham, H. Kurebayashi, R. P. Campion, B. L. Gallagher, T. Jungwirth, and A. J. Ferguson (2015), "Complementary spin-Hall and inverse spin-galvanic effect torques in a ferromagnet/semiconductor bilayer," Nature Communications 6, 6730 .

Skinner, T D, M. Wang, a. T. Hindmarch, a. W. Rushforth, A.C. Irvine, D. Heiss, H. Kurebayashi, and a. J. Ferguson (2014), "Spin-orbit torque opposing the Oersted torque in ultrathin Co/Pt bilayers," Applied Physics Letters 104, 062401.

Slavin, Andrei, and Vasil Tiberkevich (2005), "Spin wave mode excited by spin-polarized current in a magnetic nanocontact is a standing self-localized wave bullet," Physical Review Letters 95, 237201.

Slonczewski, JC (1972), "Dynamics of magnetic domain walls," International Journal of Magnetism 2, 85-97.
Šmejkal, Libor, Jakub Železný, Jairo Sinova, and Tomáš Jungwirth (2017), "Electric control of Dirac quasiparticles by spin-orbit torque in an antiferromagnet," Physical Review Letters 118, 106402.

Soleimani, M, S. Jalili, F. Mahfouzi, and N. Kioussis (2017), "Spin-orbit torque-driven magnetization switching in 2Dtopological insulator heterostructure Spin-orbit torquedriven magnetization switching in 2D-topological insulator heterostructure," Europhysics Letters 117, 37001.

Song, Qi, Hongrui Zhang, Tang Su, Wei Yuan, Yangyang Chen, Wenyu Xing, Jing Shi, Jirong Sun, and Wei Han (2017), "Observation of inverse Edelstein effect in Rashbasplit 2DEG between $\mathrm{SrTiO} 3$ and LaAlO3 at room temperature," Science Advances 3, e1602312.

Stamm, C, C. Murer, M. Berritta, J. Feng, M. Gabureac, P. M. Oppeneer, and P. Gambardella (2017), "MagnetoOptical Detection of the Spin Hall Effect in Pt and W Thin Films," Physical Review Letters 119 (8), 087203.

Stiles, M, and A. Zangwill (2002), "Anatomy of spin-transfer torque," Physical Review B 66, 014407.

Sun, J Z (2000), "Spin-current interaction with a monodomain magnetic body: A model study," Physical Review B 62 (1), 570-578.

Taguchi, Katsuhisa, Kunitaka Shintani, and Yukio Tanaka (2015), "Spin-charge transport driven by magnetization dynamics on the disordered surface of doped topological insulators," Physical Review B 92, 035425.

Takayama, A, T. Sato, S. Souma, and T. Takahashi (2011), "Giant out-of-plane spin component and the asymmetry of spin polarization in surface rashba states of bismuth thin film," Physical Review Letters 106 (16), 166401.

Tan, SG, M.B.A. Jalil, and X.-J. Liu (2007), "Local spin dynamics arising from the non-perturbative $\mathrm{SU}(2)$ gauge field of the spin-orbit coupling effect," arXiv preprint arXiV: 0705.3502v1.

Tanaka, T, H. Kontani, M. Naito, T. Naito, D. Hirashima, K. Yamada, and J. Inoue (2008), "Intrinsic spin Hall effect and orbital Hall effect in 4d and 5d transition metals," Physical Review B 77, 165117.

Tang, Peizhe, Quan Zhou, Gang Xu, and Shou-Cheng Zhang (2016), "Dirac Fermions in Antiferromagnetic Semimetal," Nature Physics 12, 1100.

Taniguchi, Tomohiro, J. Grollier, and M. D. Stiles (2015), "Spin-Transfer Torques Generated by the Anomalous Hall Effect and Anisotropic Magnetoresistance," Physical Review Applied 3 (4), 044001.

Tatara, G, Noriyuki Nakabayashi, and Kyung-Jin Lee (2013), "Spin motive force induced by Rashba interaction in the strong sd coupling regime," Physical Review B 87, 054403.

Tetienne, J, T Hingant, L J Martinez, S. Rohart, André Thiaville, L. Herrera Diez, K. Garcia, J. P. Adam, J.-V. Kim, J F. Roch, I. M. Miron, Gilles Gaudin, L. Vila, B. Ocker, D. Ravelosona, and V. Jacques (2015), "The nature of domain walls in ultrathin ferromagnets revealed by scanning nanomagnetometry," Nature Communications 6, 6733.

Thevenard, L, B. Boutigny, N. Güsken, L. Becerra, C. Ulysse, S. Shihab, A. Lemaître, J. V. Kim, V. Jeudy, and C. Gourdon (2017), "Spin transfer and spin-orbit torques in inplane magnetized (Ga,Mn)As tracks," Physical Review B 95 (5), 054422.

Thiaville, a, Y Nakatani, J Miltat, and Y Suzuki (2005), "Micromagnetic understanding of current-driven domain wall motion in patterned nanowires," Europhysics Letters 69, 990. 
Thiaville, André, Stanislas Rohart, Émilie Jué, Vincent Cros, and Albert Fert (2012), "Dynamics of Dzyaloshinskii domain walls in ultrathin magnetic films," Europhysics Letters 100, 57002 .

Thiele, A A (1973), "Steady-State Motin of Magnetic Domains," Physical Review Letters 30, 230.

Thonig, Danny, Tomas Rauch, Hossein Mirhosseini, Jurgen Henk, Ingrid Mertig, Henry Wortelen, Bernd Engelkamp, Anke B. Schmidt, and Markus Donath (2016), "Existence of topological nontrivial surface states in strained transition metals: W, Ta, Mo, and Nb," Physical Review B 94, 155132.

Tomasello, R, E. Martinez, R. Zivieri, L. Torres, M. Carpentieri, and G. Finocchio (2014), "A strategy for the design of skyrmion racetrack memories," Scientific Reports 4, 6784.

Tomasello, R, V Puliafito, E Martinez, A Manchon, M Ricci, M Carpentieri, and G Finocchio (2017), "Performance of synthetic antiferromagnetic racetrack memory : domain wall versus skyrmion," Journal of Physics D: Applied Physics 50, 325302.

Torrejon, J, G. Malinowski, M. Pelloux, R. Weil, A. Thiaville, J. Curiale, D. Lacour, F. Montaigne, and M. Hehn (2012), "Unidirectional thermal effects in current-induced domain wall motion," Physical Review Letters 109 (10), 106601.

Torrejon, Jacob, Felipe Garcia-Sanchez, Tomohiro Taniguchi, Jaivardhan Sinha, Seiji Mitani, Joo Von Kim, and Masamitsu Hayashi (2015), "Current-driven asymmetric magnetization switching in perpendicularly magnetized $\mathrm{CoFeB} / \mathrm{MgO}$ heterostructures," Physical Review B 91, 214434.

Torrejon, Jacob, Junyeon Kim, Jaivardhan Sinha, Seiji Mitani, Masamitsu Hayashi, Michihiko Yamanouchi, and Hideo Ohno (2014), "Interface control of the magnetic chirality in $\mathrm{CoFeB} / \mathrm{MgO}$ heterostructures with heavy-metal underlayers." Nature Communications 5, 4655.

Tretiakov, O, D. Clarke, Gia-Wei Chern, Ya. Bazaliy, and O. Tchernyshyov (2008), "Dynamics of Domain Walls in Magnetic Nanostrips," Physical Review Letters 100, 127204 .

Tserkovnyak, Yaroslav, Arne Brataas, and G. E. W. Bauer (2002a), "Enhanced Gilbert Damping in Thin Ferromagnetic Films," Physical Review Letters 88, 117601.

Tserkovnyak, Yaroslav, Arne Brataas, and G. E. W. Bauer (2002b), "Spin pumping and magnetization dynamics in metallic multilayers," Physical Review B 66, 224403.

Tserkovnyak, Yaroslav, D. a. Pesin, and Daniel Loss (2015), "Spin and orbital magnetic response on the surface of a topological insulator," Physical Review B 91, 041121.

Tshitoyan, V, C. Ciccarelli, A. P. Mihai, M. Ali, A. C. Irvine, T. A. Moore, T. Jungwirth, and A. J. Ferguson (2015), "Electrical manipulation of ferromagnetic NiFe by antiferromagnetic IrMn," Physical Review B 92, 214406.

Tulapurkar, a a, Y Suzuki, A Fukushima, H Kubota, H Maehara, K Tsunekawa, D D Djayaprawira, N Watanabe, and $\mathrm{S}$ Yuasa (2005), "Spin-torque diode effect in magnetic tunnel junctions." Nature 438 (7066), 339-42.

Ueda, Hiroaki T, Akihito Takeuchi, Gen Tatara, and Takehito Yokoyama (2012), "Topological charge pumping effect by the magnetization dynamics on the surface of threedimensional topological insulators," Physical Review B 85, 115110.

Ueda, Kohei, Kab-jin Kim, Yoko Yoshimura, Ryo Hiramatsu, Takahiro Moriyama, Daichi Chiba, Hironobu Tanigawa, Tetsuhiro Suzuki, Eiji Kariyada, and Teruo Ono (2014),
"Transition in mechanism for current-driven magnetic domain wall dynamics," Applied Physics Express 7, 053006.

Uhlír, V, S. Pizzini, N. Rougemaille, V. Cros, E. Jiménez, L. Ranno, O. Fruchart, M. Urbánek, G. Gaudin, J. Camarero, C. Tieg, F. Sirotti, E. Wagner, and J. Vogel (2011), "Direct observation of Oersted-field-induced magnetization dynamics in magnetic nanostripes," Physical Review B 83, 020406(R).

Uhlír, V, S Pizzini, N Rougemaille, J Novotný, V Cros, E Jiménez, G Faini, L Heyne, F Sirotti, C Tieg, A Bendounan, F Maccherozzi, R Belkhou, J Grollier, A Anane, and J Vogel (2010), "Current-induced motion and pinning of domain walls in spin-valve nanowires studied by XMCDPEEM," Physical Review B 81, 224418.

V. Baltz, A. Manchon, M. Tsoi, T. Moriyama, T. Ono, Y Tserkovnyak (2017), "Antiferromagnetic spintronics," arXiv preprint arXiv:1606.04284.

Vafek, Oskar, and Ashvin Vishwanath (2014), "Dirac Fermions in Solids-from High Tc cuprates and Graphene to Topological Insulators and Weyl Semimetals," Annual Review of Condensed Matter Physics 5, 83.

Valenzuela, S O, and M Tinkham (2006), "Direct electronic measurement of the spin Hall effect." Nature 442, 176.

Vanatka, M, J.-C. Rojas-Sánchez, J Vogel, M Bonfim, M Belmeguenai, Y Roussigné, A Stashkevich, A Thiaville, and S Pizzini (2015), "Velocity asymmetry of Dzyaloshinskii domain walls in the creep and flow regimes." Journal of Physics C 27, 326002.

Vanhaverbeke, A, A Bischof, and R Allenspach (2008), "Control of Domain Wall Polarity by Current Pulses," Physical Review Letters 101, 107202.

Verhagen, T G A, I. Leermakers, J. M. Van Ruitenbeek, and J. Aarts (2015), "Detecting Rashba fields at the interface between Co and Si oxide by ferromagnetic resonance," Physical Review B 91, 184402.

Vorobev, L E, E. L. Ivchenko, G. E. Pikus, I. I. Farbshtein, V. A. Shalygin, and A. V. Shturbin (1979), "Optical activity in tellurium induced by a current," JETP Lett. 29, 485.

Wadley, Peter, Bryn Howells, J. Elezny, Carl Andrews, Victoria Hills, Richard P. Campion, Vit Novak, K. Olejnik, F. Maccherozzi, S. S. Dhesi, S. Y. Martin, T. Wagner, J. Wunderlich, Frank Freimuth, Yuriy Mokrousov, J. Kune, J. S. Chauhan, M. J. Grzybowski, Andrew W. Rushforth, K W Edmonds, Bryan L. Gallagher, and T. Jungwirth (2016), "Electrical switching of an antiferromagnet," Science $351,587$.

Waldrop, M (2016), "More than Moore," Nature 530, 144147.

Wang, Lei, R. J H Wesselink, Yi Liu, Zhe Yuan, Ke Xia, and Paul J. Kelly (2016a), "Giant Room Temperature Interface Spin Hall and Inverse Spin Hall Effects," Physical Review Letters 116, 196602.

Wang, P, S W Jiang, Z Z Luan, L F Zhou, H F Ding, Y Zhou, X D Tao, D Wu, P Wang, S W Jiang, Z Z Luan, L F Zhou, H F Ding, Y Zhou, X D Tao, and D Wu (2016b), "Spin rectification induced by spin Hall magnetoresistance at room temperature," Applied Physics Letters 119, 112406.

Wang, Wei-Gang, Mingen Li, Stephen Hageman, and C L Chien (2012a), "Electric-field-assisted switching in magnetic tunnel junctions," Nature Materials 11, 64.

Wang, Xuhui, and Aurelien Manchon (2012), "Diffusive Spin Dynamics in Ferromagnetic Thin Films with a Rashba Interaction," Physical Review Letters 108, 117201. 
Wang, Xuhui, Christian Ortiz Pauyac, and Aurelien Manchon (2012b), "Rashba spin torque in ferromagnetic metal films," arXiv preprint arXiv:1206.6726v1.

Wang, Xuhui, Christian Ortiz Pauyac, and Aurélien Manchon (2014), "Spin-orbit-coupled transport and spin torque in a ferromagnetic heterostructure," Physical Review B 89, 054405.

Wang, Yi, Praveen Deorani, Karan Banerjee, Nikesh Koirala, Matthew Brahlek, Seongshik Oh, and Hyunsoo Yang (2015), "Topological surface states originated spin-orbit torques in Bi2Se3," Physical Review Letters 114, 257202.

Wang, Yi, Dapeng Zhu, Yang Wu, Yumeng Yang, Jiawei Yu, Rajagopalan Ramaswamy, Rahul Mishra, Shuyuan Shi, Mehrdad Elyasi, Kie-leong Teo, Yihong Wu, and Hyunsoo Yang (2017), "Room temperature magnetization switching in topological insulator-ferromagnet heterostructures by spin-orbit torques," Nature Communications 8, 1364.

Wang, Zihui, Yiyan Sun, Mingzhong Wu, Vasil Tiberkevich, and Andrei Slavin (2011), "Control of Spin Waves in a Thin Film Ferromagnetic Insulator through Interfacial Spin Scattering," Physical Review Letters 107, 146602.

Wehling, TO, A.M. Black-Schaffer, and A.V. Balatsky (2014), "Dirac materials," Advances in Physics 63 (1), 176.

Wei, Dahai, Martin Obstbaum, Mirko Ribow, C. H. Back, and Georg Woltersdorf (2014), "Spin Hall voltages from a.c. and d.c. spin currents." Nature Communications 5, 3768.

Weisheit, Martin, Sebastian Fähler, Alain Marty, Yves Souche, Christiane Poinsignon, and Dominique Givord (2007), "Electric field-induced modification of magnetism in thin-film ferromagnets." Science (New York, N.Y.) 315 (5810), 349-351.

Wen, Yan, Jun Wu, Peng Li, Qiang Zhang, Yuelei Zhao, Aurelien Manchon, John Q. Xiao, and Xixiang Zhang (2017), "Temperature dependence of spin-orbit torques in $\mathrm{Cu}-\mathrm{Au}$ alloys," Physical Review B 95 (10), 104403.

Wimmer, S, K. Chadova, M. Seemann, D. Ködderitzsch, and H. Ebert (2016), "Fully relativistic description of spin-orbit torques by means of linear response theory," Physical Review B 94, 054415.

Wolf, S A, D. D. Awschalom, R. A. Buhrman, J M Daughton, S von Molnár, M L Roukes, a Y Chtchelkanova, and D M Treger (2001), "Spintronics: a spin-based electronics vision for the future." Science (New York, N.Y.) 294 (5546), 1488-95.

Woo, S, K. Litzius, B. Krüger, M-Y. Im, L. Caretta, K. Richter, M. Mann, A. Krone, R. M. Reeve, M. Weigand, P. Agrawal, I. Lemesh, M-A. Mawass, P. Fischer, M. Kläui, and G. S. D. Beach (2016), "Observation of roomtemperature magnetic skyrmions and their current-driven dynamics in ultrathin metallic ferromagnets," Nature Materials 15, 501-506.

Woo, Seonghoon, Maxwell Mann, Aik Jun Tan, Lucas Caretta, and Geoffrey S D Beach (2014), "Enhanced spin-orbit torques in $\mathrm{Pt} / \mathrm{Co} / \mathrm{Ta}$ heterostructures," Applied Physics Letters 105, 212404.

Wu, Di, Guoqiang Yu, Ching-Tzu Chen, Seyed Armin Razavi, Qiming Shao, Xiang Li, Bingcheng Zhao, Kin L. Wong, Congli He, Zongzhi Zhang, Pedram Khalili Amiri, and Kang L. Wang (2016), "Spin-orbit torques in perpendicularly magnetized Ir22Mn78/Co20Fe60B20/MgO multilayer," Applied Physics Letters 109, 222401.

Wunderlich, Joerg, Bernd Kaestner, Jairo Sinova, and Tomas Jungwirth (2004), "Experimental discovery of the spin-
Hall effect in Rashba spin-orbit coupled semiconductor systems," arXiv preprint arXiv:cond-mat/0410295v1.

Wunderlich, Joerg, Bernd Kaestner, Jairo Sinova, and Tomas Jungwirth (2005), "Experimental observation of the spinHall effect in a two dimensional spin-orbit coupled semiconductor system," Physical Review Letters 94, 047204.

Xiao, Jiang, and G. E. W. Bauer (2012), "Spin-Wave Excitation in Magnetic Insulators by Spin-Transfer Torque," Physical Review Letters 108, 217204.

Xiao, Jiang, G. E. W. Bauer, K. Uchida, E. Saitoh, and S. Maekawa (2010), "Theory of magnon-driven spin Seebeck effect," Physical Review B 81, 214418.

$\mathrm{Xu}$, Xiaodong, Wang Yao, Di Xiao, and Tony F. Heinz (2014), "Spin and pseudospins in layered transition metal dichalcogenides," Nature Physics 10 (5), 343-350.

Yamanouchi, Michihiko, Lin Chen, Junyeon Kim, Masamitsu Hayashi, Hideo Sato, Shunsuke Fukami, Shoji Ikeda, Fumihiro Matsukura, and Hideo Ohno (2013), "Three terminal magnetic tunnel junction utilizing the spin Hall effect of iridium-doped copper," Applied Physics Letters 102 (21), 2011-2015.

Yang, H X, M. Chshiev, B. Dieny, J. H. Lee, A. Manchon, and K. H. Shin (2011), "First-principles investigation of the very large perpendicular magnetic anisotropy at $\mathrm{Fe} / \mathrm{MgO}$ and $\mathrm{Co} / \mathrm{MgO}$ interfaces," Physical Review B 84, 054401.

Yang, See-Hun, Kwang-Su Ryu, and Stuart Parkin (2015), "Domain-wall velocities of up to $750 \mathrm{~m} / \mathrm{s}$ driven by exchange-coupling torque in synthetic antiferromagnets," Nature Nanotechnology 10, 221.

Yao, Xiaofeng, Jonathan Harms, Andrew Lyle, Farbod Ebrahimi, Yisong Zhang, and Jian Ping Wang (2012), "Magnetic tunnel junction-based spintronic logic units operated by spin transfer torque," IEEE Transactions on Nanotechnology 11 (1), 120-126.

Yasuda, K, A Tsukazaki, R Yoshimi, K Kondou, K S Takahashi, Y Otani, M Kawasaki, and Y Tokura (2017), "Current-Nonlinear Hall Effect and Spin-Orbit Torque Magnetization Switching in a Magnetic Topological Insulator," Physical Review Letters 119, 137204.

Yasuda, K, A. Tsukazaki, R. Yoshimi, K. S. Takahashi, M. Kawasaki, and Y. Tokura (2016), "Large Unidirectional Magnetoresistance in a Magnetic Topological Insulator," Physical Review Letters 117, 127202.

Ye, M, S. V. Eremeev, K. Kuroda, E. E. Krasovskii, E. V. Chulkov, Y. Takeda, Y. Saitoh, K. Okamoto, S. Y. Zhu, K. Miyamoto, M. Arita, M. Nakatake, T. Okuda, Y. Ueda, K. Shimada, H. Namatame, M. Taniguchi, and A. Kimura (2012), "Quasiparticle interference on the surface of Bi2Se3 induced by cobalt adatom in the absence of ferromagnetic ordering," Physical Review B 85, 205317.

Yokoyama, Takehito, Jiadong Zang, and N. Nagaosa (2010), "Theoretical study of the dynamics of magnetization on the topological surface," Physical Review B 81, 241410.

Yoon, Jungbum, Seo-Won Lee, Jae Hyun Kwon, Jong Min Lee, Jaesung Son, Xuepeng Qiu, Kyung-Jin Lee, and Hyunsoo Yang (2017), "Anomalous spin-orbit torque switching due to field-like torque-assisted domain wall reflection," Science Advances 3 (4), e1603099.

Yoshimura, Yoko, and Tomohiro Koyama (2014), "Effect of spin Hall torque on current-induced precessional domain wall motion," Applied Physics Express 7, 033005.

You, Long, OukJae Lee, Debanjan Bhowmik, Dominic Labanowski, Jeongmin Hong, Jeffrey Bokor, and Sayeef Salahuddin (2015), "Switching of perpendicularly polarized 
nanomagnets with spin orbit torque without an external magnetic field by engineering a tilted anisotropy," Proceedings of the National Academy of Sciences 112 (33), 10310-10315.

Young, Steve M, and Charles L. Kane (2015), "Dirac Semimetals in Two Dimensions," Physical Review Letters 115, 126803.

Yu, Guoqiang, Pramey Upadhyaya, Yabin Fan, Juan G. Alzate, Wanjun Jiang, Kin L Wong, So Takei, Scott A Bender, Li-Te Chang, Ying Jiang, Murong Lang, Jianshi Tang, Yong Wang, Yaroslav Tserkovnyak, Pedram Khalili Amiri, and Kang L Wang (2014a), "Switching of perpendicular magnetization by spin-orbit torques in the absence of external magnetic fields." Nature Nanotechnology 9, 548.

$\mathrm{Yu}$, Guoqiang, Pramey Upadhyaya, Xiang Li, Wenyuan Li, Se Kwon Kim, Yabin Fan, Kin L. Wong, Yaroslav Tserkovnyak, Pedram Khalili Amiri, and Kang L. Wang (2016a), "Room-Temperature Creation and Spin-Orbit Torque Manipulation of Skyrmions in Thin Films with Engineered Asymmetry," Nano Letters 16, 1981.

Yu, Guoqiang, Pramey Upadhyaya, Kin L. Wong, Wanjun Jiang, Juan G. Alzate, Jianshi Tang, Pedram Khalili Amiri, and Kang L. Wang (2014b), "Magnetization switching through spin-Hall-effect-induced chiral domain wall propagation," Physical Review B 89, 104421.

Yu, Jiawei, Xuepeng Qiu, William Legrand, and Hyunsoo Yang (2016b), "Large spin-orbit torques in Pt/Co-Ni/W heterostructures," Applied Physics Letters 109, 042403.

Yuan, Zhe, and Paul J. Kelly (2016), "Spin-orbit-coupling induced torque in ballistic domain walls: Equivalence of charge-pumping and nonequilibrium magnetization formalisms," Physical Review B 93, 224415.

Zelezný, J (2017), "symcode," .

Železný, J, H Gao, Aurélien Manchon, Frank Freimuth, Yuriy Mokrousov, J. Zemen, J. Mašek, Jairo Sinova, and T. Jungwirth (2017), "Spin-orbit torques in locally and globally non-centrosymmetric crystals: Antiferromagnets and ferromagnets," Physical Review B 95, 014403.

Železný, J, H. Gao, K. Výborný, and J. Zemen (2014), "Relativistic Néel-Order Fields Induced by Electrical Current in Antiferromagnets," Physical Review Letters 113, 157201.

Zhang, C, S Fukami, H Sato, F Matsukura, and H Ohno (2015a), "Spin-orbit torque induced magnetization switching in nano-scale Ta/CoFeB/MgO," Applied Physics Letters 107, 012401.

Zhang, H J, S Yamamoto, Y Fukaya, M Maekawa, H Li, A Kawasuso, T Seki, E Saitoh, and K Takanashi (2014), "Current-induced spin polarization on metal surfaces probed by spin-polarized positron beam." Scientific reports 4,4844 .

Zhang, Jia, Julian P. Velev, Xiaoqian Dang, and Evgeny Y. Tsymbal (2016a), "Band structure and spin texture of Bi2Se3 3d ferromagnetic metal interface," Physical Review B 94, 014435.

Zhang, S, P. Levy, and A. Fert (2002), "Mechanisms of SpinPolarized Current-Driven Magnetization Switching," Physical Review Letters 88, 236601.

Zhang, S, and Z. Li (2004), "Roles of Nonequilibrium Conduction Electrons on the Magnetization Dynamics of Ferromagnets," Physical Review Letters 93, 127204.

Zhang, W, Wei Han, S.-H. Yang, Yan Sun, Yang Zhang, Binghai Yan, and Stuart S P Parkin (2016b), "Giant facetdependent spin-orbit torque and spin Hall conductivity in the triangular antiferromagnet IrMn3," Science Advances 2, e1600759-e1600759.

Zhang, Wei, Matthias B Jungfleisch, Wanjun Jiang, John E Pearson, and Axel Hoffmann (2015b), "Spin pumping and inverse Rashba-Edelstein effect in $\mathrm{NiFe} / \mathrm{Ag} / \mathrm{Bi}$ and $\mathrm{NiFe} / \mathrm{Ag} / \mathrm{Sb}$," Journal of Applied Physics 117, $17 \mathrm{C} 727$.

Zhang, Wei, Joseph Sklenar, Bo Hsu, Wanjun Jiang, Matthias B. Jungfleisch, Jiao Xiao, Frank Y. Fradin, Yaohua Liu, John E. Pearson, John B. Ketterson, Zheng Yang, and Axel Hoffmann (2016c), "Research Update: Spin transfer torques in permalloy on monolayer MoS2," APL Materials 4, 032302.

Zhang, Weifeng, Wei Han, Xin Jiang, See Hun Yang, and Stuart S.P. Parkin (2015c), "Role of transparency of platinumferromagnet interfaces in determining the intrinsic magnitude of the spin Hall effect," Nature Physics 11, 496.

Zhang, Wenxu, Bin Peng, Fangbin Han, Qiuru Wang, Wee Tee Soh, Chong Kim Ong, and Wanli Zhang (2016d), "Separating inverse spin Hall voltage and spin rectification voltage by inverting spin injection direction," Applied Physics Letters 108, 102405.

Zhang, Xichao, Motohiko Ezawa, and Yan Zhou (2015d), "Magnetic skyrmion logic gates: conversion, duplication and merging of skyrmions." Scientific reports 5, 9400.

Zhang, Xichao, Yan Zhou, and Motohiko Ezawa (2015e), "Magnetic bilayer-skyrmions without skyrmion Hall effect," Nature Communications 7, 10293.

Zhao, Zhengyang, Angeline K Smith, Mahdi Jamali, and Jian-ping Wang (2017), "External-Field-Free Spin Hall Switching of Perpendicular Magnetic Nanopillar with a Dipole-Coupled Composite Structure," arXiv preprint arXiv:1603.09624.

Zutic, Igor, Jaroslav Fabian, and S Das Sarma (2004), "Spintronics: Fundamentals and applications," Review of Modern Physics 76, 323. 